\title{
EFFICIENT TECHNIQUES FOR COOPERATIVE SPECTRUM SENSING IN COGNITIVE RADIO NETWORKS
}

\section{Lamiaa Khalid}

BSc, Ain Shams University, Cairo, Egypt, 2000 MASc, Ryerson University, Toronto, Ontario, 2008

\author{
A Dissertation \\ Presented to the School of Graduate Studies at \\ Ryerson University \\ in partial fulfilment of the \\ requirements for the degree of \\ Doctor of Philosophy \\ in the Program of Electrical and Computer Engineering
}

Toronto, Ontario, Canada, 2014

(C)Lamiaa Khalid, 2014 


\section{AUTHOR'S DECLARATION FOR ELECTRONIC SUBMISSION OF A DISSERTATION}

I hereby declare that I am the sole author of this dissertation. This is a true copy of the dissertation, including any required final revisions, as accepted by my examiners.

I authorize Ryerson University to lend this dissertation to other institutions or individuals for the purpose of scholarly research.

I further authorize Ryerson University to reproduce this dissertation by photocopying or by

other means, in total or in part, at the request of other institutions or individuals for the purpose of scholarly research.

I understand that my dissertation may be made electronically available to the public. 


\title{
Abstract \\ Efficient Techniques for Cooperative Spectrum Sensing in \\ Cognitive Radio Networks
}

\author{
(C)Lamiaa Khalid, 2014 \\ Doctor of Philosophy \\ Electrical and Computer Engineering \\ Ryerson University
}

In this thesis, we focus on two important design aspects of cooperative spectrum sensing (CSS) in cognitive radio networks which are the selection criterion of cooperating secondary users and the fusion technique for combining their local sensing decisions. We propose a novel adaptive user-group assignment algorithm that addresses the problem of sensing accuracyefficiency trade-off in group-based CSS with heterogeneous cooperating secondary users. The performance of the proposed algorithm is bounded by $4.2 \%$ of the optimal solution. Through extensive simulations, we demonstrate that the proposed algorithm can effectively improve the performance of CSS in terms of the opportunistic throughput, sensing overhead and the number of sensing rounds needed to discover an available channel. Considering the different detection performance of cooperating secondary users, we propose a novel reliabilitybased decision fusion scheme in which a weight is assigned to each secondary user's local decision based on its reliability. Since the knowledge of the local probabilities of detection and false alarm for each secondary detector may not be known in practice, we employ a counting process to estimate those probabilities based on past global and local decisions. We then formulate the problem of minimizing the network probability of sensing error and develop a dual search algorithm, based on a non-linear Lagrangian approach, to solve the formulated problem. Our simulation results show that the dual algorithm converges to the optimal value with zero duality gap using few number of iterations. We also show that the probability of error is reduced by $18 \%$ and $88 \%$ compared to the OR and AND fusion rules, 
respectively, when the number of secondary users is eight. We then address the practical concern of secondary users reporting correlated local decisions to the fusion center. For this scenario, we formulate the problem of minimizing the network probability of sensing error as an optimization problem and employ the genetic algorithm to jointly find the optimal $K^{*}$-out-of- $M$ fusion rule and the optimal local threshold for a certain correlation index. Simulation results show that the network probability of sensing error degrades as the degree of correlation between cooperating secondary users increases. We also study the problem of multiband cooperative joint detection in the presence of sensing errors due to time offset. We derive the aggregate opportunistic throughput and aggregate interference to primary users for multiband cooperative joint detection in the presence of time offset. Our numerical results demonstrate the negative impact of the time offset on the aggregate opportunistic throughput of multiband cooperative joint detection. 


\section{Acknowledgements}

All praises are due to Almightily Allah, the most Gracious, and the most Merciful. I thank Him for all the bounties and blessings He bestowed upon me and for granting me the strength and patience to complete this work.

I would like to express my sincerest gratitude to my supervisor, Professor Alagan Anpalagan, for his patient guidance, warm encouragement and invaluable advice throughout the course of my PhD study. He was always understanding of the difficulties and the challenges one inevitably faces during the seemingly never ending PhD study. His positive outlook and endless support inspired me and gave me the confidence and motivation needed to finish this work. His careful and insightful reviews contributed enormously to the production of this thesis. It was truly a great privilege working with him.

I would like to thank my thesis committee members, Professor Dimitri Androutsos, Professor Lian Zhao and Professor Abdolreza Abhari, for taking the time and effort to review my thesis and provide me with their insightful comments. My deep appreciations go out to Professor Elvino Sousa, University of Toronto, for agreeing to act as my external examiner and provide me with his valuable comments. I would also like to acknowledge the administrative staff at the school of graduate studies and the department of electrical and computer engineering at Ryerson university for all the instances in which their assistance helped me along the way. Furthermore, I acknowledge that this research would not have been possible without the financial assistance of the Natural Sciences and Engineering Research Council of Canada, Government of Ontario and Ryerson University.

Thanks are also due to my friends and colleagues in the radio resource management radio access and networking (RRM \& RAN) research group present and past, I am lucky to be a part of this group where a team spirit truly prevails. Our group discussions and their insightful feedback have helped in many occasions. I would especially like to thank Dr. Muhammad Naeem and Kandasamy Illanko for their help on mathematics and programming.

The most special thanks I accord to my beloved husband, Osama Bazan, who was amaz- 
ingly patient, supportive, compassionate and understanding and who helped me through this sometimes overwhelming $\mathrm{PhD}$ journey. He was always willing to listen and discuss ideas with me that greatly contributed to the improvement of the work presented in this thesis. I benefited numerous times from his critical thinking and deep knowledge. I would not be where I am today without his support and constant encouragement. Many special thanks to my family for all their support and understanding, especially my mother who is always a great source of love and motivation. I could never thank her enough for everything she has done for me and I hope I always make her proud. I would also like to thank my parents in-law for their love and encouragement.

My deepest love and gratitude is to my grandmother, who helped me grow into the person I am today. Thank you for all the little and great things you have done for me, you will forever live in my heart. Last but not least, I would like to thank my beloved sons, Zeyad and Eiad, who are my constant source of joy and happiness and whose presence ease all life difficulties and make it worthwhile. 


\section{Contents}

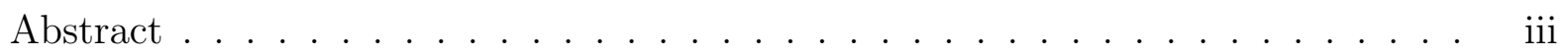

Acknowledgements ........................ . . .

List of Tables .................................. xii

List of Figures . . . . . . . . . . . . . . . . . . . . xvi

List of Acronyms . . . . . . . . . . . . . . . . . . . . . xvi

List of Symbols . . . . . . . . . . . . . . . . . . xvi

1 Introduction $\quad 1$

1.1 Cognitive Radio Technology . . . . . . . . . . . . . . . . . 3

1.2 Thesis Motivation . . . . . . . . . . . . . . . . 6

1.3 Research Contributions . . . . . . . . . . . . . . . . 8

1.4 Thesis Outline . . . . . . . . . . . . . . . . . 11

2 Spectrum Sensing for Cognitive Radio 14

2.1 Introduction . . . . . . . . . . . . . . . . . . . 14

2.2 Hypothesis Testing . . . . . . . . . . . . . . . . . . 15

2.3 Primary Transmitter Detection _. . . . . . . . . . . . . . . . 18

2.4 Sensing Techniques . . . . . . . . . . . . . . . . 20

2.4.1 Matched Filter Detection . . . . . . . . . . . . . . . 20

2.4 .2 Energy Detection . . . . . . . . . . . . . . . 22

2.4.3 Cyclostationary Feature Detection . . . . . . . . . . . . . 22 
2.4 .4 Other Sensing Techniques _ . . . . . . . . . . . . . . . . . 23

2.5 Cooperative Spectrum Sensing $(\mathrm{CSS}) \ldots \ldots \ldots$

2.5.1 Cooperation Architecture . . . . . . . . . . . . . . 25

2.5 .2 Fusion Schemes . . . . . . . . . . . . . . . . . . 27

2.5.3 Cooperative User Selection . . . . . . . . . . . . . . . . . . 30

2.5.4 Cooperation Overhead . . . . . . . . . . . . . . . . . . . 32

2.5 .5 Sensing Errors . . . . . . . . . . . . . . . . . . . 34

2.5.6 Multiband Spectrum Sensing _. . . . . . . . . . . . . . 36

2.6 Chapter Summary . . . . . . . . . . . . . . . . . . . . . . . . 37

\section{Adaptive User Assignment for Group-based Cooperative Spectrum Sens-} $\begin{array}{lr}\text { ing } & 39\end{array}$

3.1 Introduction $\ldots \ldots \ldots \ldots \ldots \ldots$

3.2 Related Work . . . . . . . . . . . . . . . . . . . . . . . . . 40

3.3 System Model . . . . . . . . . . . . . . . . . . . . . . . . . . . . 43

3.3 .1 Energy Detection . . . . . . . . . . . . . . . . . . . 44

3.3.2 Primary User Traffic Activity Model . . . . . . . . . . . . . . . . 46

3.4 Adaptive Group-based Cooperative Sensing Scheme . . . . . . . . . . . . . 48

3.4 .1 Fixed Rate Transmission . . . . . . . . . . . . . . . . . . . . . 51

3.4.2 Variable Rate Transmission . . . . . . . . . . . . . . . . 54

3.4.3 Problem Formulation . . . . . . . . . . . . . . . . . . . . . 55

3.5 Adaptive Assignment Algorithm . . . . . . . . . . . . . . . . . . 57

3.5 .1 Channel Sorting . . . . . . . . . . . . . . . . . 58

3.5 .2 User Assignment . . . . . . . . . . . . . . . . . . . . . . . 58

3.5 .3 Group Assignment . . . . . . . . . . . . . . . . . . . . . . . 59

3.5 .4 Complexity Analysis . . . . . . . . . . . . . . . . . 60

3.6 Performance Evaluation $\ldots \ldots \ldots 2$

3.6 .1 Fixed Rate Transmission . . . . . . . . . . . . . . . . . . . 62 
3.6.2 Variable Rate Transmission . . . . . . . . . . . . . . . 66

3.7 Chapter Summary . . . . . . . . . . . . . . . . . . . . . . . 75

4 Reliability-based Decision Fusion Scheme for CSS Based on Past Decisions 77

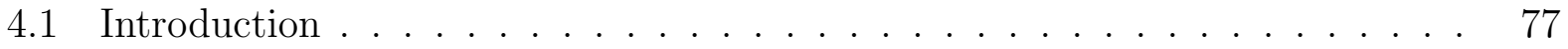

4.2 Related Work . . . . . . . . . . . . . . . . . . . 78

4.3 Problem Formulation . . . . . . . . . . . . . . . . . . . . . 83

4.4 Probability Estimation using Past Decisions . . . . . . . . . . . . . . . 87

4.5 Proposed Reliability-based Decision Fusion Scheme . . . . . . . . . . . 89

4.6 Dual Search Algorithm . . . . . . . . . . . . . . . . . . . . . . . . . . 90

4.7 Performance Evaluation $\ldots \ldots \ldots 9$.

4.7 .1 Simulation Results . . . . . . . . . . . . . . . . . . 93

4.7.2 Dual Search Based Results . . . . . . . . . . . . . . . . . . . . . . . . 98

4.8 Chapter Summary . . . . . . . . . . . . . . . . . . . . . . . . . 101

5 Decision Fusion Scheme for CSS with Correlated Local Decisions 104

5.1 Introduction . . . . . . . . . . . . . . . . . . . . 104

5.2 Related Work . . . . . . . . . . . . . . . . . . . . . . . . 105

5.3 Correlation Model . . . . . . . . . . . . . . . . . . . . . . . . 108

5.4 Decision Fusion with Correlated Local Decisions . . . . . . . . . . . . . 111

5.4.1 General $K$-out-of- $M$ Fusion Rule . . . . . . . . . . . . . . . . 111

5.4 .2 Majority Voting (MV) Fusion Rule . . . . . . . . . . . . . . . . 112

5.4 .3 OR Fusion Rule . . . . . . . . . . . . . . . . . . . . . . . . . . . 112

5.4 .4 AND Fusion Rule . . . . . . . . . . . . . . . . . . . . . . . . 113

5.5 CSS Based on Neyman-Pearson Criterion . . . . . . . . . . . . . . 113

5.6 Correlation-based $K^{*}$-out-of- $M$ Optimum Rule . . . . . . . . . . . . 120

5.6 .1 Problem Formulation . . . . . . . . . . . . . . . . . . . . . 121

5.6.2 Genetic Algorithm (GA) Based Solution . . . . . . . . . . . . 122 
5.7 Performance Evaluation _. . . . . . . . . . . . . . . . . . . . . 123

5.7 .1 Numerical Results . . . . . . . . . . . . . . . . . . . . . . . . 124

5.7 .2 GA Based Results . . . . . . . . . . . . . . . . . . . . . 125

5.8 Chapter Summary . . . . . . . . . . . . . . . . . . . . . . . . . . . . . . . . 128

6 Multiband Cooperative Joint Detection with Sensing Errors 130

6.1 Introduction . . . . . . . . . . . . . . . . . 130

6.2 Related Work . . . . . . . . . . . . . . . . . . . . . . . 131

6.3 Multiband Cooperative Joint Detection . . . . . . . . . . . . . . . . . 134

6.4 Sensing Errors due to Time Offset . . . . . . . . . . . . . . . . . . 138

6.4 .1 Problem Formulation . . . . . . . . . . . . . . . . . . . . . . 140

6.4.2 Derivation of Threshold Bounds . . . . . . . . . . . . . . . . . . 143

6.5 Performance Evaluation . . . . . . . . . . . . . . . . . . . . 144

6.6 Chapter Summary . . . . . . . . . . . . . . . . . . . . . . . . . 149

7 Conclusions and Future Work 151

7.1 Conclusions . . . . . . . . . . . . . . . . . . . . . . . . 152

7.2 Future Work . . . . . . . . . . . . . . . . . . . . . 155

$\begin{array}{lr}\text { Appendix } & 158\end{array}$

$\begin{array}{ll}\text { A Some Concepts in Optimization } & 158\end{array}$

A.1 Classification of Optimization Problems . . . . . . . . . . . . 158

A.2 Classes of Complexity . . . . . . . . . . . . . . . . . . . . 159

A.3 Computational Complexity of Algorithms . . . . . . . . . . . . . . . 161

B List of Publications $\quad 164$

B.1 Journal Publications . . . . . . . . . . . . . . . . . . . . . . . . . . 164

B.2 Conference Publications _. . . . . . . . . . . . . . . . . . . . . 164 
Bibliography

166 


\section{List of Tables}

2.1 Comparison of Spectrum Sensing Techniques. . . . . . . . . . . . . . . 24

4.1 Values of the Indictor Function $Z_{1,1}^{k, j} \ldots \ldots \ldots \ldots$

4.2 Dual Search Algorithm Parameters. . . . . . . . . . . . . . . . . . . . 98

4.3 Performance Results of the Dual Search Algorithm. . . . . . . . . . . . . 99

5.1 GA Parameters. . . . . . . . . . . . . . . . . . . . . . . 124

5.2 Mean and Standard Deviation of the Network Probability of Sensing Error over 20 Runs. . . . . . . . . . . . . . . . . . . . . . . . 127

A.1 Table of Common Time Complexities as a Function of the Input Size m. . 162 


\section{List of Figures}

1.1 Spectrum hole concept. . . . . . . . . . . . . . . . . . . 2

1.2 Thesis outline. . . . . . . . . . . . . . . . . . . 13

2.1 Trade-off between probability of false alarm and probability of miss-detection. 16

2.2 Interference range of a cognitive radio. . . . . . . . . . . . . . . . 19

2.3 Classification of spectrum sensing techniques. . . . . . . . . . . . . . 21

2.4 Parallel fusion architecture. . . . . . . . . . . . . . . . . 26

2.5 Decentralized fusion architecture. . . . . . . . . . . . . . . . 27

3.1 An illustration of a cognitive radio network. . . . . . . . . . . . . 44

3.2 Block diagram of energy detector. . . . . . . . . . . . . . . 45

3.3 An ON-OFF model for primary users' channel usage. . . . . . . . . . . . . . 47

3.4 Frame structure for secondary users. . . . . . . . . . . . . . . . . . . . . . . 48

3.5 Illustration of sequential cooperative spectrum sensing scheme. . . . . . . . 49

3.6 Illustration of the group-based cooperative sensing scheme. . . . . . . . . . 50

3.7 Comparision of throughput efficiency with different number of cooperating

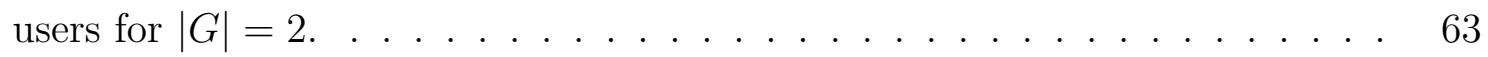

3.8 Throughput efficiency versus $K$ for $|G|=4$. . . . . . . . . . . . . . 64

3.9 Throughput efficiency versus $|G|$ for $K=2|G| \ldots \ldots$. . . . . . . . . . . . . . 65

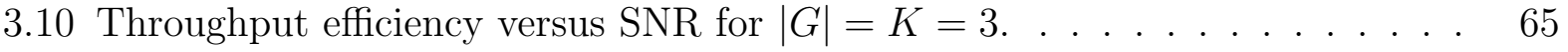

3.11 Throughput efficiency versus number of cooperating users for $|G|=2$. . . . 67 
3.12 Throughput efficiency versus number of cooperating users for $|G|=4$. . . .

3.13 Average opportunistic throughput versus number of cooperating users for $|G|=4 \ldots \ldots \ldots \ldots \ldots \ldots$

3.14 Average sensing overhead versus number of cooperating users for $|G|=4$. . 69

3.15 Probability mass function of the number of sensing rounds for the proposed and non-adaptive schemes for $|G|=4$ and $K=4 \ldots \ldots \ldots$. . . . . . 70

3.16 Probability mass function of the number of sensing rounds for the proposed and non-adaptive schemes for $|G|=4$ and $K=8$. . . . . . . . . . . . . 70

3.17 Throughput efficiency versus number of groups for $K=2|G|$. . . . . . . . . 71

3.18 Probability mass function of the number of sensing rounds for the proposed and non-adaptive schemes for $|G|=3$ and $K=6$. . . . . . . . . . . . . . 72

3.19 Probability mass function of the number of sensing rounds for the proposed and non-adaptive schemes for $|G|=5$ and $K=10 \ldots \ldots$. . . . . . . . . 72

3.20 Average opportunistic throughput versus channel availability for $|G|=4$ and $K=6 \ldots \ldots \ldots \ldots \ldots$

3.21 Average sensing overhead versus channel availability for $|G|=4$ and $K=6 . \quad 74$

3.22 Throughput efficiency versus SNR for $|G|=4$ and $K=6 \ldots \ldots$. . . . . . 74

4.1 Frame structure for secondary users with fixed observation window. . . . . . 83

4.2 Weighted cooperative spectrum sensing scheme. . . . . . . . . . . . . 84

4.3 Network probability of detection versus the window size for different values

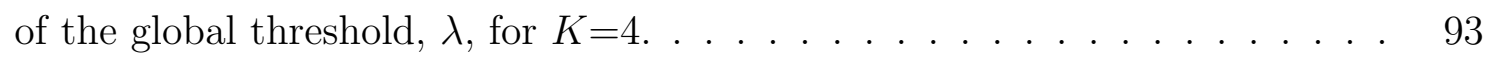

4.4 Network probability of false alarm versus the window size for different values of the global threshold, $\lambda$, for $K=4 \ldots \ldots \ldots \ldots \ldots$

4.5 Network probability of sensing error versus the window size for different values of the global threshold, $\lambda$, for $K=4 \ldots \ldots$. . . . . . . . . . . . . 94

4.6 Network probability of detection versus the window size for different values of the SNR for $\lambda=0$ and $K=4 \ldots \ldots \ldots \ldots \ldots$ 
4.7 Network probability of false alarm versus the window size for different values of the SNR for $\lambda=0$ and $K=4 \ldots \ldots \ldots$. . . . . . . . . . . . . . . 96

4.8 Comparison of the receiver operating characteristic curves for the proposed scheme and the OR and AND fusion schemes. . . . . . . . . . . . . . 97

4.9 Network probability of sensing error versus the global threshold, $i$, for different number of secondary users for $J=25 . \ldots \ldots$. . . . . . . . .

4.10 Comparision of the minimum probability of sensing error for the dual search algorithm and the exhaustive search for different number of secondary users for $\beta=0.9 \ldots \ldots \ldots \ldots \ldots \ldots \ldots$

4.11 Comparision of the minimum probability of sensing error for the proposed scheme and the OR and AND fusion schemes for different number of secondary users for $\beta=0.9$.

4.12 Minimum probability of sensing error versus SNR for different number of secondary users for $\beta=0.9 \ldots \ldots \ldots$. . . . . . . . . . . . . . 101

5.1 ROC curves $\left(P_{D}\right.$ vs. $\left.P_{F}\right)$ for different correlation index for majority voting fusion rule with $M=5$ and $\mathrm{SNR}=-10 \mathrm{~dB}$. . . . . . . . . . . . . . 115

5.2 Comparison of ROC curves $\left(P_{D}\right.$ vs. $\left.P_{F}\right)$ for different number of secondary users, $K$, when $\rho=0.05 \ldots \ldots \ldots \ldots \ldots \ldots \ldots$

5.3 Comparison of ROC curves $\left(P_{D}\right.$ vs. $\left.P_{F}\right)$ for different number of secondary users, $K$, when $\rho=0.2 \ldots \ldots \ldots \ldots \ldots \ldots$

5.4 Network probability of detection versus correlation index for OR, AND and majority voting rules when $M=5$ for $P_{F}=0.1$ and $\mathrm{SNR}=-10 \mathrm{~dB}$.

5.5 Network probability of detection versus correlation index for OR, AND and majority voting rules when $M=10$ for $P_{F}=0.1$ and SNR $=-10 \mathrm{~dB} . \ldots . . .118$

5.6 Network probability of detection versus SNR for different values of $\rho$ when $P_{F}=0.1$ and $M=5 \ldots \ldots \ldots \ldots \ldots \ldots$ 
5.7 Network probability of false alarm versus number of users $K$, with $M=30$, $\mathrm{SNR}=-10 \mathrm{~dB}$ and $P_{D}=0.9 \ldots \ldots \ldots \ldots \ldots \ldots$

5.8 Network probability of false alarm versus SNR for different number of users $K$ with $\rho=0.05$ and $P_{D}=0.9 . \ldots \ldots \ldots \ldots \ldots$

5.9 Network probability of sensing error versus local sensing threshold for different

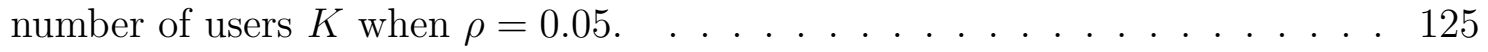

5.10 Network probability of sensing error versus the number of users $K$ when $\beta=0.9 .126$

5.11 Network probability of sensing error versus the number of users $K$ for different

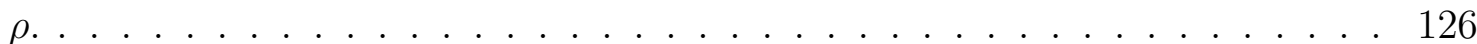

5.12 Evolution of the network probability of sensing error using GA algorithm when

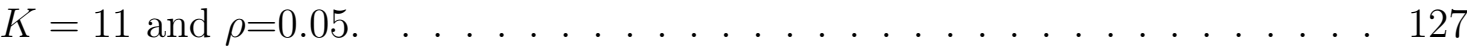

6.1 An illustration of the occupancy of a multiband channel. . . . . . . . . . . . 134

6.2 Block diagram of multiband joint detection for wideband spectrum sensing. . 135

6.3 Comparision of maximum aggregate opportunistic throughput for the cooperative and non-cooperative schemes. . . . . . . . . . . . . . . . . 145

6.4 Network probability of false alarm for each subchannel $l$ for $\varepsilon=1.25$. . . . . . 146

6.5 Network probability of miss-detection for each subchannel $l$ for $\varepsilon=1.25$. . . . 146

6.6 Maximum aggregate opportunistic throughput versus average SNR for $\varepsilon=2 . \quad 147$

6.7 Maximum aggregate opportunistic throughput versus the aggregate interference for different normalized time offsets. . . . . . . . . . . . . . . . . . 148

6.8 Network probability of false alarm for each subchannel $l$ for different normalized time offsets and $\varepsilon=4 \ldots \ldots \ldots$. . . . . . . . . . . . . . . 148

6.9 Network probability of miss-detection for each subchannel $l$ for different normalized time offsets and $\varepsilon=4 \ldots \ldots \ldots \ldots \ldots$. . . . . . . . . . . . . . . . . . . . 


\section{List of Acronyms}

\begin{tabular}{ll} 
AWGN & Additive White Gaussian Noise \\
CR & Cognitive Radio \\
CSI & Channel State Information \\
CSS & Cooperative Spectrum Sensing \\
DSA & Dynamic Spectrum Access \\
EGC & Equal Gain Combining \\
FCC & Federal Communications Commission \\
GLF & Generalized Lagrangian Function \\
GLRT & Generalized Likelihood Ratio Test \\
i.i.d & independent and identically distributed \\
LRT & Likelihood Ratio Test \\
MRC & Maximum Ratio Combining \\
MV & Majority Voting \\
NP & Neyman-Pearson \\
pdf & probability density function \\
PSD & Power Spectral Density \\
PU & Primary User \\
RF & Radio Frequency \\
SIR & Signal-to-Interference Ratio \\
SNR & Signal-to-Noise Ratio \\
SU & Secondary User \\
\hline
\end{tabular}




\section{List of Symbols}

a Transition rate of primary channel state from $\mathrm{ON}$ to $\mathrm{OFF}$

$a_{l} \quad$ Transition rate of primary suchannel $l$ state from $\mathrm{ON}$ to $\mathrm{OFF}$

A Aggregate interference to the primary user

$A_{u} \quad$ Aggregate interference to primary user $u$

$\bar{A}_{l} \quad$ Expected interference to primary users on subchannel $l$

$A_{0}^{\prime} \quad$ The set of secondary users declaring hypothesis $H_{0}$ is true

$A_{1}^{\prime} \quad$ The set of secondary users declaring hypothesis $H_{1}$ is true

$b \quad$ Transition rate of primary channel state from OFF to ON

$b_{l} \quad$ Transition rate of primary subchannel $l$ state from OFF to ON

$B_{l} \quad$ Busy state for subchannel $l$

$c_{l} \quad$ Cost of interfering with the primary user on subchannel $l$

$C_{i, j} \quad$ Cost of declaring hypothesis $H_{i}$ when hypothesis $H_{j}$ is true

$d_{k} \quad$ Local decision of secondary user $k$

$d_{0} \quad$ Global decision of fusion center

$d_{l, k} \quad$ Local decision on subchannel $l$ by secondary user $k$

$d_{k, j}^{\prime} \quad$ Local decision of secondary user $k$ at frame $j$

$d_{0, j}^{\prime} \quad$ Global decision of fusion center at frame $j$

$D \quad$ Vector of secondary users' local descisions

$D_{l} \quad$ Vector of secondary users' local decisions on subchannel $l$

$D_{1}^{\prime} \quad$ Communication range of primary system

$D_{2}^{\prime} \quad$ Interference range

$e_{k} \quad$ Weighted decision of secondary user $k$

$f(v) \quad$ Function of variable $v$

$f_{\nu} \quad$ Probability density function of random variable $\nu$

$f_{\nu}^{l} \quad$ Probability density function of random variable $\nu$ on subchannel $l$

F Opportunistic secondary throughput 
$F_{l} \quad$ Expected opportunistic secondary throughput on subchannel $l$

$g_{i} \quad$ The group of secondary users sensing channel $i$

G The set of all groups

$G^{q} \quad$ The set of groups sensing in round $q$

$h\left(D^{\prime}\right) \quad$ Channel gain at distance $D^{\prime}$ from transmitter

$h_{k} \quad$ Channel gain at secondary user $k$

$h_{k}^{l} \quad$ Channel gain of channel $l$ at secondary user $k$

$H_{0} \quad$ Null hypothesis

$H_{1} \quad$ Alternate hypothesis

$H_{0, l} \quad$ Null hypothesis for subchannel $l$

$H_{1, l} \quad$ Alternate hypothesis for subchannel $l$

$H_{l}^{\prime} \quad$ Channel gain of subchannel $l$

$H_{l, k}^{\prime} \quad$ Channel gain of subchannel $l$ at secondary user $k$

$I_{l} \quad$ Idle state for subchannel $l$

$J \quad$ Window size

$K \quad$ Number of secondary users

L Number of channels (subchannels)

$L_{p}^{\prime} \quad$ Generalized Lagrangian function

$\mathbf{m}_{(K, 2)} \quad K$-bit binary vector

$m \quad$ Problem size

$M \quad$ Total number of secondary users

$M^{\prime} \quad$ Number of transmission modes

$N \quad$ Number of observation samples

$O \quad$ Sensing overhead

$P($.$) \quad Probability of an event$

$p_{q} \quad$ Probability of discovering at least one channel at the $q^{\text {th }}$ sensing rounds

$P_{d} \quad$ Local probability of detection 
$P_{d, k} \quad$ Local probability of detection for secondary user $k$

$P_{d, k}^{l} \quad$ Local probability of detection for secondary user $k$ on channel (subchannel) $l$

$P_{f} \quad$ Local probability of false alarm

$P_{f, k} \quad$ Local probability of false alarm for secondary user $k$

$P_{f, k}^{l} \quad$ Local probability of false alarm for secondary user $k$ on channel (subchannel) $l$

$P_{m} \quad$ Local probability of miss-detection

$P_{B} \quad$ Steady state busy probability

$P_{D} \quad$ Network probability of detection

$P_{D}^{l} \quad$ Network probability of detection on subchannel $l$

$P_{E} \quad$ Network probability of sensing error

$P_{F} \quad$ Network probability of false alarm

$P_{F}^{l} \quad$ Network probability of false alarm on subchannel $l$

$P_{M}^{l} \quad$ Network probability of miss-detection on subchannel $l$

$P_{q} \quad$ Probability of discovering at least one channel after $q$ sensing rounds

$P_{s} \quad$ Probability of successfully discovering a channel

$P_{I} \quad$ Steady state idle probability

$P_{u}^{\prime} \quad$ Transmit power of primary user

$P_{s}^{\prime} \quad$ Transmit power of secondary user

$P_{b}^{\prime} \quad$ Background interference power at primary receiver

Q Total number of sensing rounds

$r_{k} \quad$ Reliability of secondary user $k$

$R \quad$ Transmission rate of a secondary user

$R_{l} \quad$ Transmission rate of a secondary user on channel (subchannel) $l$

$R_{l, k} \quad$ Transmission rate of secondary user $k$ on channel $l$

$\mathbf{R}$ Vector of transmission rates corresponding to different transmission modes

$s(n)$ Primary user signal at time instant $n$

$S_{l} \quad$ Primary signal at subchannel $l$ 
$S_{l, u}^{\prime} \quad$ The set of subchannels used by primary user $u$

$T \quad$ Total frame time of secondary users

$T_{s} \quad$ Sensing time of secondary users

$T_{t} \quad$ Transmission time of secondary users

$\Delta t \quad$ Time offset

$U^{\prime} \quad$ Number of primary users

$v_{k}(n) \quad$ Additive noise at secondary user $k$ at time instant $n$

$V_{l, k}(n) \quad$ Additive noise at secondary user $k$ on subchannel $l$ at time instant $n$

$w_{k} \quad$ Weight assigned to secondary user $k$ local decision

W Channel bandwidth

$x(n) \quad$ Observed signal at time instant $n$

$x_{k}(n) \quad$ Observed signal at secondary user $k$ at time instant $n$

$X_{l, k}(n)$ Observed signal at secondary user $k$ on subchannel $l$ at time instant $n$

x Observation vector

$Y \quad$ Test statistics

$Y_{k} \quad$ Test statistics of secondary user $k$

$Y_{l, k} \quad$ Test statistics of secondary user $k$ on subchannel $l$

$Z_{x, y}^{k, j} \quad$ Global-local decision indictor function

$\alpha_{l} \quad$ Upper bound on the network probability of miss-detection for subchannel $l$

$\boldsymbol{\alpha} \quad$ vector of upper bounds on the network probability of miss-detection for all subchannels

$\beta \quad$ Lower bound on the network probability of detection

$\beta_{l}^{\prime} \quad$ Upper bound on the network probability of false alarm for subchannel $l$

$\boldsymbol{\beta}^{\prime} \quad$ vector of upper bounds on the network probability of false-alarm for all subchannels

$\gamma_{k} \quad$ Local threshold of secondary user $k$

$\gamma_{l, k} \quad$ Local threshold of secondary user $k$ on subchannel $l$

$\gamma_{\min , l} \quad$ Lower bound on the local threshold for subchannel $l$

$\gamma_{\text {max }, l} \quad$ Upper bound on the local threshold for subchannel $l$ 
$\gamma_{N P} \quad$ Local threshold for the Neyman Pearson test

$\gamma_{l}^{\prime} \quad$ Local threshold on subchannel $l$

$\Gamma_{k}^{\prime} \quad$ Signal-to-noise ratio at secondary user $k$

$\Gamma_{l, k}^{\prime} \quad$ Signal-to-noise ratio for subchannel $l$ at secondary user $k$

$\Gamma^{\prime} \quad$ Vector of signal-to-noise ratios of different secondary users

$\Gamma_{\text {eff }} \quad$ Throughput efficiency

$\delta($.$) \quad Delta dirac function$

$\epsilon_{y}^{x} \quad$ Correlation coefficient between $y$ secondary local decisions under hypothesis $H_{x}$

$\varepsilon \quad$ Upper bound on the aggregate interference to primary users

$\varepsilon_{u} \quad$ Upper bound on the aggregate interference to primary user $u$

$\eta_{i, q} \quad$ Group-sensing round indicator function

$\lambda \quad$ Global threshold of the fusion center

$\mu \quad$ Lagrange multiplier

$\xi_{i, k} \quad$ User-group indicator function

$\rho \quad$ Correlation index

$\rho^{x} \quad$ Correlation index under hypothesis $H_{x}$

$\rho_{k_{1}, k_{2}}^{x} \quad$ Correlation coefficient between variables $k_{1}$ and $k_{2}$ under hypothesis $H_{x}$

$\sigma_{v}^{2} \quad$ Noise variance

$\sigma_{s}^{2} \quad$ Primary signal variance

$\varphi \quad$ Indicator function for the $K$-out-of- $M$ system

$\Psi \quad$ Test statistics of fusion center

$\Phi_{\nu}(\omega)$ Characteristic function of random variable $\nu$ 


\section{Chapter 1}

\section{Introduction}

Driven by the proliferation of new wireless services and applications, as well as the steadily increasing number of wireless users, the demand for radio spectrum has increased dramatically. The government regulatory agencies employ an inflexible spectrum management approach by granting each operator an exclusive license to operate in a certain frequency band. With most of the prime radio frequency spectrum already exclusively assigned, it is becoming exceedingly hard to find vacant bands to either deploy new services or enhance existing ones. However, this spectrum scarcity is mainly due to inefficient fixed frequency allocations rather than a physical shortage in the spectrum. In fact, the federal communications commission (FCC) has reported the temporal and geographic variations in spectrum utilization to range from $15 \%$ to $85 \%$ [1]. This inefficiency in the spectrum usage necessitates a new communication paradigm to exploit the existing wireless spectrum opportunistically.

Dynamic spectrum access (DSA) has been proposed as an alternative policy to allow the radio spectrum to be more efficiently utilized [2]. Using DSA, a portion of the spectrum can be licensed to one or more users, which are called primary users; however, the use of that spectrum is not exclusively granted to these licensed users, although they have higher priority

in using it. The unlicensed users, which are referred to as secondary users, are allowed to opportunistically utilize the unused licensed bands, commonly referred to as "white spaces" 
or "spectrum holes", as long as the primary users' transmissions can be adequately protected. By doing so, the radio spectrum can be reused in an opportunistic manner or shared all the time which can significantly improve the spectrum utilization efficiency [3]. The spectrum hole concept is illustrated in Figure 1.1.

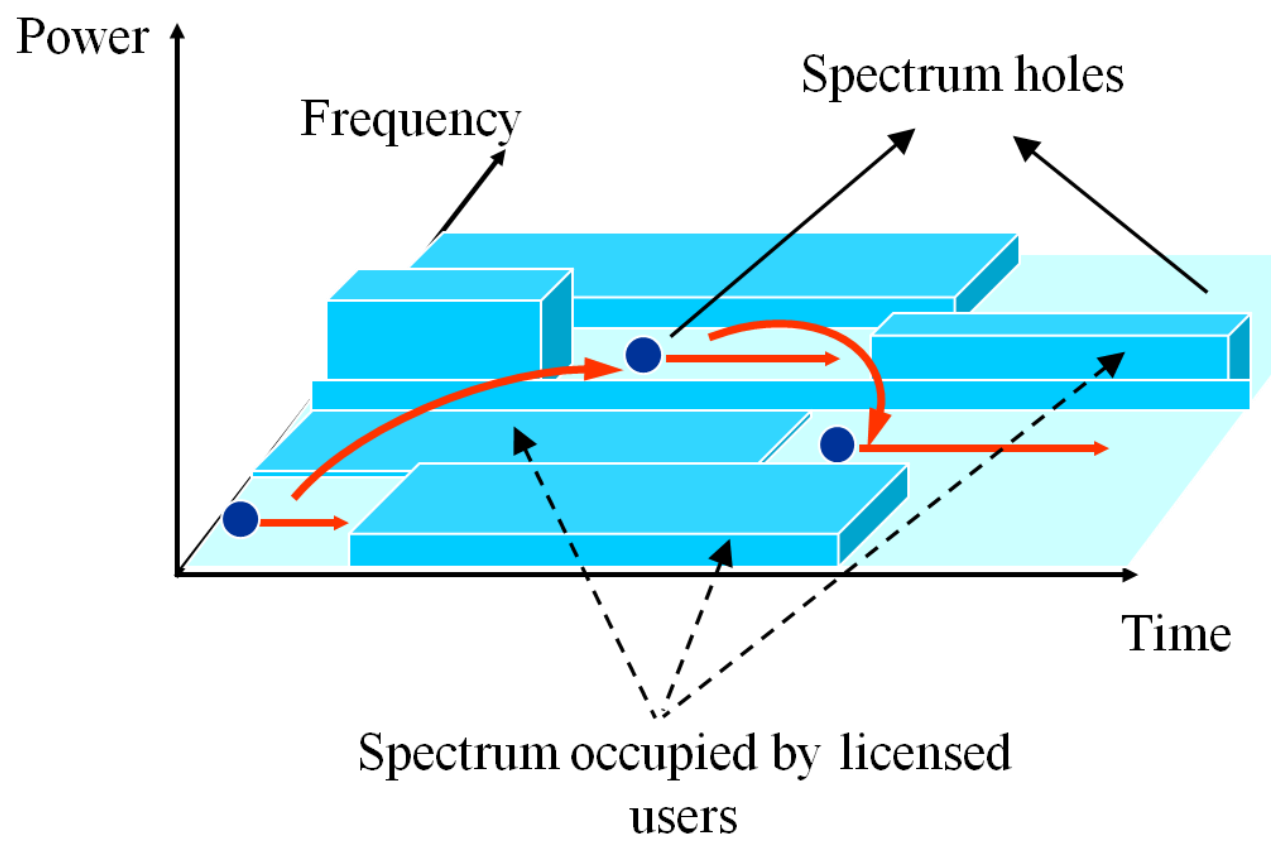

Figure 1.1: Spectrum hole concept.

The federal communications commission has already expressed its interest in permitting unlicensed access to white spaces in the television (TV) bands [4]. This interest stems in part from the great propagation characteristics of the TV bands and their relatively predictable spatio-temporal usage characteristics. To reliably identify the white spaces, some methods that the secondary users can employ are: geolocation combined with access to database, beacons, spectrum sensing or a combination of any of those methods $[5,6]$. In the geolocation method, primary users register the relevant data such as their location and transmit power as well as expected duration of usage at a centralized database. Secondary users then have to access this database to determine the availability of white spaces at their location. In the beacon method, secondary users only transmit if they receive a control signal (beacon) 
identifying vacant channels within their service areas. Without reception of this control signal, no transmissions are permitted by the secondary users. The aforementioned methods require some modifications to the current licensed systems and their deployment is costly. In addition, with these methods, secondary devices will need additional connectivity in a different band in order to be able to access the database [5] or a dedicated standardized channel will be needed to broadcast the beacons [6]. In the spectrum sensing method, secondary users autonomously detect the presence of the primary signals and only use the channels that are not used by the primary users. Due to its low infrastructure cost and its compatibility with the primary systems, we adopt the spectrum sensing method in this thesis and we provide a detailed overview of this method in Chapter 2.

\subsection{Cognitive Radio Technology}

The key enabling technology of DSA is the cognitive radio (CR) technology. A cognitive radio system is a radio system which is aware of its operational and geographical environment, established policies, and its internal state. Moreover, it is able to dynamically and autonomously adapt its operational parameters and protocols and to learn from its previous experience [1]. Cognitive radios are enabled by the rapid and significant advancements in radio technologies (e.g., software-defined radios, frequency agility, power control, etc.), and can be characterized by the utilization of disruptive techniques such as wideband spectrum sensing, real-time spectrum allocation and acquisition, and real-time measurement dissemination [7].

As an enabling technology for DSA, the ultimate objective of the cognitive radio is to

efficiently utilize the available spectrum through cognitive capability and reconfigurability. These two main characteristics of CR can be defined as follows [8]:

- Cognitive Capability: through real-time interaction with the radio environment, the spectrum holes at a specific time or location can be identified. The tasks required 
for adaptive operation in open spectrum are $[8,9]$ :

- Spectrum sensing: determine which portions of the spectrum are available for transmission and detect the presence of licensed users when a CR user operates in a licensed band by estimating the interference levels of the radio environment.

- Spectrum analysis: includes the estimation of channel state information (CSI); and the prediction of channel capacity for use by the CR transmitter.

- Spectrum decision: a CR determines the data rate, the transmission mode, and the bandwidth of the transmission. The appropriate spectrum band is then chosen according to the spectrum characteristics and user's requirements.

- Reconfigurability: a CR can be programmed to transmit and receive on a variety of frequencies and using different access technologies supported by its hardware design. There are several reconfigurable parameters that can be incorporated into the CR such as operating frequency, modulation, transmission power and communication technology. According to the spectrum characteristics, these parameters can be reconfigured such that the $\mathrm{CR}$ is switched to a different spectrum band, the transmitter and receiver parameters are reconfigured and the appropriate communication protocol parameters and modulation schemes are used.

In this thesis, we consider a cognitive radio network where multiple secondary users are equipped with cognitive radios and are allowed to access the unused licensed bands that belong to multiple primary users. In order to protect the primary users' transmissions from the adverse effects of secondary users' interference, white spaces across frequency, time and space should be reliably identified. Based on the type of available network side information along with the regulatory constraints, cognitive radio networks seek to underlay, overlay, or interweave their signals with those of existing primary users without significantly impacting their communication [10]. The underlay paradigm allows secondary users to operate if the interference caused to the primary users' transmissions is below a given threshold. In 
overlay systems, the secondary users employ sophisticated signal processing and coding to maintain or improve the communication of primary users while also obtaining some additional bandwidth for their own communication. In interweave systems, the secondary users opportunistically exploit the instances of silence of primary users to transmit their signals. Since mitigating the interference through opportunistic spectrum access requires little or even no collaboration between primary and secondary users, spectrum interweave has found its way to practical implementations and is adopted by a number of spectrum regulatory agencies worldwide [11] and this is the one adopted in this thesis.

Spectrum sensing is crucial to allowing efficient dynamic spectrum access in cognitive radio networks [12-14]. However, due to the effects of multipath fading and shadowing, the signal-to-noise ratio (SNR) of the received primary signal can be extremely small and a secondary user may not be able to distinguish between a deeply faded band and an idle one. Since receiver sensitivity indicates the capability of detecting weak signals, the sensitivity requirement of the secondary user's receiver may end up being too stringent. In order to mitigate these effects, secondary users often cooperate for spectrum sensing.

The main idea of cooperative spectrum sensing is to enhance the sensing performance by exploiting the spatial diversity in the observations of spatially located secondary users [15-19]. In cooperative sensing, secondary users can send their sensing information to a centralized unit, called a fusion center, for making a combined decision [14]. Cooperative sensing techniques can be grouped into soft decision and hard decision combining schemes according to which kind of information is forwarded to the fusion center. In the soft decision combining schemes, secondary users send their test statistics calculated from their local observations. On the other hand, in the hard decision combining schemes, secondary users only send their individual binary local decisions to the fusion center. Having multiple cooperating secondary users increases diversity by providing multiple measurements of the signal and therefore, guarantees a better detection performance. Consequently, the diversity gain achieved through cooperative spectrum sensing improves the overall detection sensitivity 
without imposing higher sensitivity requirements on individual secondary users [20]. A less stringent sensitivity requirement is particularly appealing from the implementation point of view due to the reduced hardware cost and complexity. The performance improvement due to spatial diversity is called cooperative gain. However, cooperative gain is not limited to improved detection performance and relaxed sensitivity requirement. For instance, since it is not possible for a single radio to transmit on a licensed band and sense it simultaneously, sensing has to be interleaved with data transmission $[15,21]$. Therefore, if the sensing time can be reduced due to cooperation, secondary users will have more time for data transmission so as to improve their opportunistic throughput. In this case, the improved opportunistic throughput is also a part of the cooperative gain.

\subsection{Thesis Motivation}

From the above discussion, it is apparent that well-designed techniques for cooperative spectrum sensing (CSS) can significantly contribute to improving the achievable cooperative gain. However, CSS can incur cooperation overhead and the achievable cooperative gain can be impacted by many factors. This motivates us to focus in this thesis on how to effectively leverage the achievable cooperative gain without compromising the performance of cooperative sensing by the incurred cooperation overhead. Previous works on CSS rely heavily on the idea of sequential spectrum sensing in which multiple secondary users cooperate to sense a single channel in each sensing period. However, this traditional cooperative sensing technique may greatly limit the overall sensing efficiency, which refers to the number of channels or spectrum access opportunities that can be discovered in each sensing period. Therefore, it is important to investigate other cooperative sensing techniques that can enhance the sensing efficiency by simultaneously detecting multiple distinct channels within each sensing period. This enhancement can result in higher opportunistic throughput for secondary users and therefore, higher cooperative gain. This motivates us to study in this thesis two sensing 
techniques that enable the sensing of multiple channels in each sensing period, namely, the parallel cooperative spectrum sensing technique described in Chapter 3 and the multiband joint detection technique described in Chapter 6 .

The selection of secondary users for CSS plays a key role in determining the performance of cooperative sensing. In most of the previous work on CSS, it is assumed that the secondary users have the same detection performance. However, in practical scenarios, different secondary users will have different detection performance. In addition, most existing models for cooperative sensing focus on cooperative gain while cooperation overhead has been largely ignored. However, proper modeling of cooperation overhead can reveal realistic achievable cooperative gain which makes it an important research issue to address. Therefore, one of the motivations of this work is to study the problem of selecting secondary users with independent local decisions for cooperation according to their detection performance to help improve the cooperative gain and address cooperation overhead issues.

Since the local detection precision of a secondary user is closely related to its detection performance, secondary users with different detection performance will have different local decision reliability. Therefore, for cooperative spectrum sensing, the final global decision at fusion centre will be affected by the reliability of the secondary users' local decisions. Based on this argument, we find it necessary for the fusion center to incorporate the reliability of each secondary user's local decision when making a final decision to achieve a more accurate fusion result. Therefore, it is of interest to design a decision fusion technique for heterogeneous secondary users that incorporates adaptive weights for the local decisions reported to the fusion center.

Typically, a strong correlation exists among secondary users that are located close to each other which means that their local decisions are also correlated. For secondary users with correlated local decision, the effect of correlation on the performance of CSS needs to be considered. Therefore, we study in this thesis a correlation model that can quantify the degree of correlation between secondary users' decisions. We are interested in finding the 
optimal decision fusion rule to combine the results from secondary users with correlated local decision according to the degree of correlation between their local decisions.

In most of the previous works on sensing and cooperation, it is assumed that the primary signal appears from the beginning of a sensing period to facilitate analysis. It is further assumed that the state of the primary user's signal will not change from the time the sensing was performed until the time the channel is opportunistically used by the secondary user. Ignoring this time offset may lead to sensing errors that will result in degraded performance of the cooperative sensing scheme. This motivates us to investigate the performance of CSS in the presence of sensing errors which may result due to factors such as reporting delay, asynchronous sensing or synchronization errors.

\subsection{Research Contributions}

The main objective of this research is to design efficient techniques for cooperative spectrum sensing that are capable of discovering multiple spectrum access opportunities in a single sensing period to increase the achievable cooperative gain while limiting the incurred cooperation overhead and sensing errors. Specifically, we focus on two important aspects of cooperative spectrum sensing which are cooperating user selection and reliable fusion of sensing results. We address the problem of sensing accuracy-efficiency trade-off in cooperative spectrum sensing through the selection of independent heterogeneous secondary users for parallel cooperative spectrum sensing such that multiple channels can be sensed simultaneously by a group of those users. To further enhance the performance improvement, we address the problem of fusing the local decisions of heterogeneous secondary users by taking the reliability of those local decisions into consideration. When secondary users' decisions are correlated, we derive an optimal $K^{*}$-out-of- $M$ fusion rule that minimizes the probability of sensing error for a certain degree of correlation between the local decisions. In the problem

of multiband joint detection, we address the problem of reliably fusing the sensing results 
of cooperating users by considering the effect of sensing errors that may occur during fusion due to the presence of time offset between local sensing and decision fusion of local results.

The key contributions of this thesis are summarized as follows:

- Designing a novel adaptive user-group assignment algorithm for group-based cooperative spectrum sensing with heterogeneous cooperating secondary users to achieve a trade-off between the sensing efficiency and sensing accuracy.

- Analytically derive an expression for the average opportunistic throughput and average sensing overhead for the group-based cooperative sensing scheme when the secondary users have heterogeneous sensing ability in terms of the sensing accuracy while incorporating the cost of using multiple sensing rounds to find a vacant channel.

- Formulate the throughput efficiency maximization problem for heterogeneous secondary users as a non-linear binary programming problem that is generally $N P$ hard.

- Propose an efficient heuristic adaptive assignment algorithm to solve the formulated optimization problem in which the heterogeneous secondary users are adaptively assigned to groups based on their probabilities of detection and show that the proposed algorithm can achieve comparable performance to the optimal solution with much lower computational complexity.

- Examine the effect of different key parameters such as the number of groups, the number of secondary users and the channel availability on the performance of the proposed algorithm.

- Designing a novel reliability-based decision fusion scheme that uses past information about local and global sensing decisions to estimate the reliability of the sensing decision made by each secondary user in the form of a weight factor that is then taken into account when making the final decision at the fusion center. 
- Formulate the problem of minimizing the network probability of sensing error with heterogeneous secondary users subject to a limit on the network probability of detection as a constrained non-linear integer programming problem.

- Develop a dual search algorithm, employing a non-linear Lagrangian approach, to solve the formulated optimization problem.

- Evaluate the performance of the developed dual search algorithm using extensive simulations and show that it is effective in solving the optimization problem as it converges to the optimal value with zero duality gap when the Lagrange multiplier is larger than a certain threshold.

- Examine the effect of different key parameters such as the number of users, window size and the signal-to-noise ratio on the performance of the proposed reliabilitybased scheme.

- Developing an optimal decision fusion rule that considers the correlation between the cooperating secondary users' local decisions.

- Derive the network probabilities of detection and false alarm for the $K$-out-of- $M$ fusion rule, when the secondary users' local decisions are correlated under both hypothesis, employing a correlation model that quantifies the degree of correlation by a single correlation index.

- Optimize the network probability of false alarm with constraint on the network probability of detection when the local decisions are correlated and show that there is an optimal value of $K$ that satisfies this Neyman-Pearson criterion for each correlation index.

- Formulate the problem of minimizing the network probability of sensing error under the correlation model used in our analysis as a mixed integer non-linear programming problem. 
- Employ the genetic algorithm to find the optimal number of secondary users, $K^{*}$, and the local threshold that jointly minimize the network probability of sensing error for a certain degree of correlation.

- Analyzing the impact of sensing errors due to time offset on the performance of the multiband cooperative joint detection framework.

- Investigate the performance of cooperative wideband spectrum sensing, based on multiband joint detection, when hard decision combining is employed at the fusion center.

- Consider a probability-based combining scheme for multiband cooperative joint detection that takes into account the effect of sensing errors due to time offset.

- Derive both the aggregate opportunistic throughput of secondary users and aggregate interference to primary users for multiband cooperative joint detection in the presence of time offset by taking into consideration the statistical information of the licensed band occupancy.

- Evaluate and discuss the impact of sensing errors due to time offset on the performance of multiband cooperative joint detection.

\subsection{Thesis Outline}

The remainder of this thesis is organized as follows. In the next chapter, we present a brief overview of spectrum sensing techniques and cooperative spectrum sensing. We highlight the most important aspects of cooperative spectrum sensing such as various fusion schemes and cooperative user selection criteria. We further discuss some of the limiting factors of cooperative spectrum sensing, namely, cooperation overhead and sensing errors. In Chapter 3, we present the general system model considered in this thesis. We then formulate the throughput efficiency maximization problem for group-based cooperative sensing with 
heterogeneous secondary users and propose an adaptive user-group assignment algorithm to solve the formulated optimization problem. To further enhance the performance of cooperative spectrum sensing, we propose, in Chapter 4, a novel decision fusion scheme that considers the reliability of the sensing decisions made by heterogeneous secondary users when making a final decision at the fusion center. We employ a probability estimation technique, that uses past information about local and global decisions, to estimate the reliability of the sensing decision made by each secondary user. We then formulate the problem of minimizing the network probability of sensing error and develop a dual search algorithm to solve the formulated problem. In Chapter 5, we consider secondary users with correlated local decisions and present the correlation model. We then formulate the problem of minimizing the probability of sensing error when cooperating secondary users' decisions are correlated and employ the genetic algorithm to solve the formulated optimization problem. Having addressed the problem of user selection and reliable fusion for narrowband spectrum sensing, we consider, in Chapter 6, multiband joint detection and investigate the performance improvement that can be achieved by hard decision cooperation. We then consider a probability-based combining scheme that takes into account the effect of sensing errors due to time offset. We also investigate the impact of time offset on multiband cooperative joint detection. Finally, we conclude this thesis in Chapter 7 and present some directions for future work. The outline of the thesis is given in Figure 1.2 which also depicts where each of the contributions fits. 


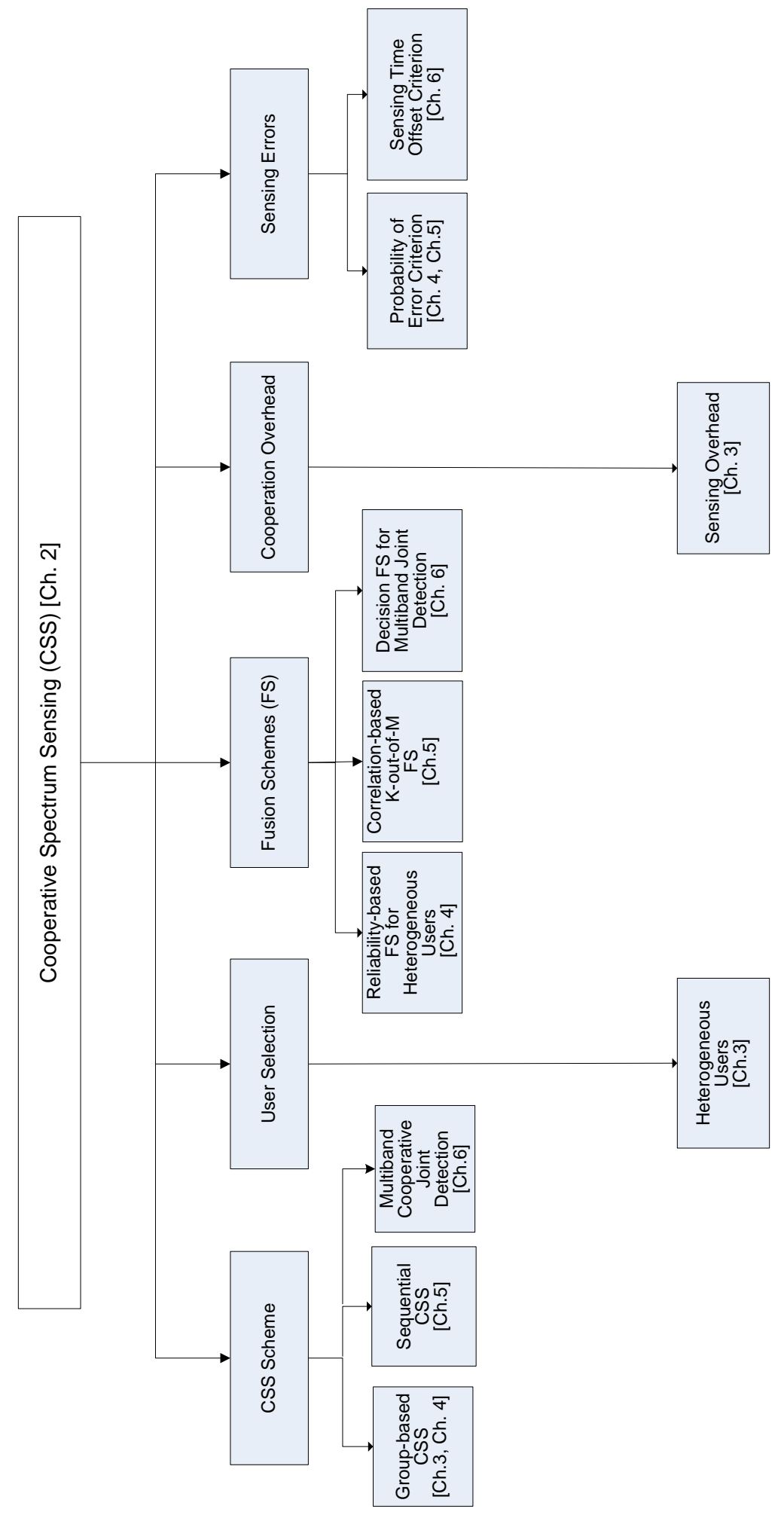

Figure 1.2: Thesis outline. 


\section{Chapter 2}

\section{Spectrum Sensing for Cognitive Radio}

\section{$2.1 \quad$ Introduction}

Spectrum sensing is the most important task among others for the effective operation of cognitive radio networks. Spectrum sensing enables the capability of a cognitive radio to measure, learn, and be aware of the radio's operating environment, such as the spectrum availability and interference status. Availability of radio spectrum varies depending on time, frequency and location resulting in spectrum access opportunities. Secondary users can use the available idle spectrum in an opportunistic manner. Spectrum sensing helps secondary users to achieve this objective by identifying the available spectrum reliably and rapidly. Spectrum sensing also helps secondary users to detect the presence of primary signals to protect the primary users' transmission. It also helps in quickly determining if the primary users have become active in the bands used by secondary users so that those bands can be vacated immediately. This is important for ensuring that the interference caused to the primary users' transmissions remains below a permitted level. Moreover, detection of other secondary users may be necessary as well for co-existence with other secondary networks. Recent surveys on spectrum sensing and related issues can be found in $[12,19,22]$.

The spectrum sensing problem is traditionally formulated as a binary hypothesis testing 
problem as described in section 2.2. To identify the idle spectrum and protect the primary users' transmissions, different local spectrum sensing techniques have been proposed for individual secondary users based on hypothesis testing. Some of the most common spectrum sensing techniques for the detection of primary users' transmissions for cognitive radio networks are discussed in section 2.4.

\subsection{Hypothesis Testing}

A key task in spectrum sensing is to decide whether the spectrum is idle or busy. The spectrum sensing problem is traditionally formulated as a binary hypothesis test [23]. The null hypothesis denoted by $H_{0}$ corresponds to the absence of the primary user's transmission, i.e., the received signal being only noise. On the other hand, the alternative hypothesis denoted by $H_{1}$ indicates that the primary user's transmission is present, i.e., the received signal contains the primary signal along with noise. In case the hypotheses have no unknown parameters, the hypotheses are called simple. If there are unknown or unspecified parameters, then the hypotheses are called composite. As an example, a binary hypothesis test for detecting the primary user's transmission in an additive white Gaussian noise (AWGN) channel is given by

$$
x(n)= \begin{cases}v(n), & H_{0} \\ h(n) s(n)+v(n), & H_{1}\end{cases}
$$

where $x(n)$ is the received signal at the $n^{\text {th }}$ time instant, $h(n)$ is the channel gain at the $n^{\text {th }}$ time instant. The primary user's transmitted signal is denoted by $s(n)$ and, $v(n)$ is the AWGN noise. In most practical cases, a test statistic $Y$ is computed from the observation vector $\mathbf{x}=[x(1), x(2), . ., x(N)]$ containing $N$ observation samples, and detection is based on comparing the test statistic $Y$ to the threshold $\gamma$. If the test statistic is greater than the threshold, i.e., $Y>\gamma$ then $H_{1}$ is declared true. Otherwise, $H_{0}$ is declared true. Two main performance metrics that are crucial in the design of spectrum sensing techniques are the 
probability of miss-detection, $P_{m}$, and the probability of false alarm, $P_{f}$. The probability of miss-detection is defined as the probability that the detector declares the absence of a primary user $(\mathrm{PU})$ transmission (decide $H_{0}$ ), when $\mathrm{PU}$ transmission is actually present ( $H_{1}$ is true). The probability of false alarm is defined as the probability that the detector declares the presence of PU transmission (decide $H_{1}$ ), when PU transmission is actually absent $\left(H_{0}\right.$ is true). Therefore, we represent the probabilities of miss-detection and false alarm, respectively, as [24]

$$
P_{m}=P\left(H_{0} \mid H_{1}\right)=P\left(Y \leq \gamma \mid H_{1}\right)
$$

and

$$
P_{f}=P\left(H_{1} \mid H_{0}\right)=P\left(Y>\gamma \mid H_{0}\right)
$$

The trade-off between the probability of false alarm and miss-detection is depicted in Figure 2.1. In the figure, the likelihood distributions for the absence and presence of the primary user's signal are both assumed to be normally distributed with respective means $\mu_{1}$ and $\mu_{2}$ and the variances of the distributions are taken to be equal.

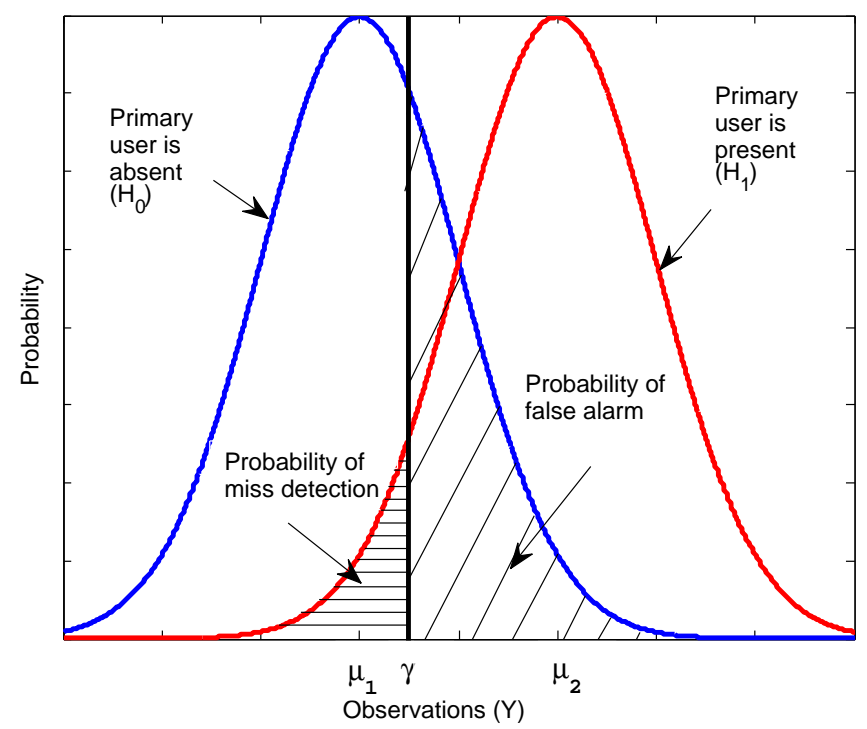

Figure 2.1: Trade-off between probability of false alarm and probability of miss-detection. 
It is clear from the above discussion that we need the probability of detection, $P_{d}=1-P_{m}$, to be high as it indicates the level of protection of the primary users' transmissions from the interfering secondary users' transmissions. On the other hand, low probabilities of false alarm are necessary in order to maintain high opportunistic secondary throughput, since a false alarm would prevent the unused bands from being accessed by secondary users leading to inefficient spectrum usage.

There are two basic hypothesis testing methods in spectrum sensing: the NeymanPearson (NP) test $[25,26]$ and the Bayes test $[27,28]$. In an NP test, the objective is to maximize the detection probability, $P_{d}$, given the constraint on the probability of false alarm, $P_{f}$. Based on the signal detection problem in (2.1), it can be shown that the NP test is equivalent to the likelihood ratio test (LRT) [19]. The LRT test statistics is given by

$$
Y_{l r t}=\prod_{n=1}^{N} \frac{P\left(x(n) \mid H_{1}\right)}{P\left(x(n) \mid H_{0}\right)} \underset{H_{0}}{\stackrel{H_{1}}{\gtrless}} \gamma_{l r t} .
$$

In a Bayes test, the objective is to minimize the expected cost called the Bayes risk given by

$$
B R=\sum_{i=0}^{1} \sum_{j=0}^{1} C_{i j} P\left(H_{i} \mid H_{j}\right) P\left(H_{j}\right)
$$

where $C_{i j}$ and $P\left(H_{i} \mid H_{j}\right)$ are, respectively, the cost and the probability of declaring $H_{i}$ when $H_{j}$ is true, and $P\left(H_{j}\right)$ is the prior probability of hypothesis $H_{j}, i, j \in\{0,1\}$. In other words, the Bayes risk to be minimized is the sum of all possible costs weighted by the probabilities of two incorrect detection cases (false alarm $\left(P\left(H_{1} \mid H_{0}\right)\right.$ and miss-detection $\left(P\left(H_{0} \mid H_{1}\right)\right)$ and two correct detection cases. With the knowledge of the prior probabilities $P\left(H_{1}\right)$ and $P\left(H_{0}\right)$, the LRT of a Bayes test can be represented as

$$
Y_{l r t}=\prod_{n=1}^{N} \frac{P\left(x(n) \mid H_{1}\right)}{P\left(x(n) \mid H_{0}\right)} \underset{H_{0}}{\gtrless} \frac{P\left(H_{0}\right)\left(C_{10}-C_{00}\right)}{P\left(H_{1}\right)\left(C_{01}-C_{11}\right)}=\gamma_{B T} .
$$

For the particular case of the binary loss function, $C_{i i}=0$ and $C_{i j}=1$ for $i \neq j$, the 
Bayes risk, $B R$, is equal to the probability of error, $P_{E}$. Therefore,

$$
\begin{aligned}
P_{E} & =P\left(H_{1} \mid H_{0}\right) P\left(H_{0}\right)+P\left(H_{0} \mid H_{1}\right) P\left(H_{1}\right) \\
& =P_{f} P\left(H_{0}\right)+\left(1-P_{d}\right) P\left(H_{1}\right) .
\end{aligned}
$$

As mentioned earlier, if the distributions of the received signal under the two hypotheses depend on unknown parameters, then the detection problem becomes a composite hypothesis testing problem. One of the approaches to composite hypothesis testing that does not require prior knowledge of the unknown parameters is the generalized likelihood ratio test (GLRT) $[19,28]$. In GLRT, the unknown parameters are determined by the maximum likelihood estimates. Although GLRT is not an optimal test, it is robust and easy to implement. The GLRT is given by

$$
Y_{\text {glrt }}=\prod_{n=1}^{N} \frac{\max _{\theta_{1}} P\left(x(n) \mid \theta_{1}, H_{1}\right)}{\max _{\theta_{0}} P\left(x(n) \mid \theta_{0}, H_{0}\right)} \underset{H_{0}}{\stackrel{H_{1}}{\gtrless}} \gamma_{\text {glrt }},
$$

where $\theta_{1}$ and $\theta_{0}$ are the unknown random parameters.

\subsection{Primary Transmitter Detection}

The transmitter detection model is based on the detection of weak signals from a primary transmitter through the local observations of secondary users. This model has a wider applicability due to its compatibility with the licensed systems. The main drawback of the primary transmitter sensing model is its reliance on the detection of primary transmitters to infer the availability of white spaces while the interference happens at the primary receivers. As such, a detection margin has to be included in order to protect primary receivers [6].

When the primary system employs bursty transmission, the secondary user can detect the empty time slots and multiplex its signal over them without causing any performance degradation at the primary receivers. On the other hand, when the primary system employs continuous transmission, the secondary user has to estimate the interference it generates at 


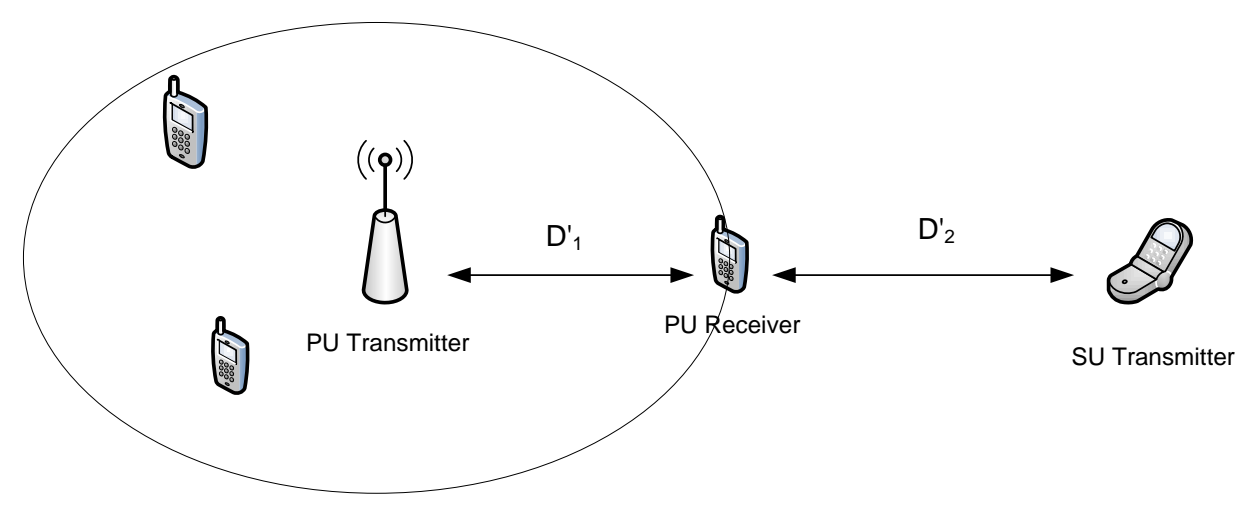

Figure 2.2: Interference range of a cognitive radio.

the primary receivers by using signal level measurements. If the transmitter of the secondary user is far from the primary receiver, depending on the signal-to-interference ratio (SIR) limit at the receiver of the primary user supplied by the regulatory bodies, both the primary user and the secondary user could transmit data simultaneously [6,29]. In this case, the interference range is defined as the minimum distance that a secondary transmitter should be away from the primary receiver such that it does not cause harmful interference at this receiver. Figure 2.2 shows the primary receiver located at a distance $D_{1}^{\prime}$ from the primary transmitter and $D_{2}^{\prime}$ is the interference range. The interference range will depend on the SU transmitted power and the primary receiver's interference tolerance and can be obtained from $[30]$

$$
\mathrm{SIR}=\frac{P_{u}^{\prime} h\left(D_{1}^{\prime}\right)}{P_{s}^{\prime} h\left(D_{2}^{\prime}\right)+P_{b}^{\prime}},
$$

where $P_{u}^{\prime}$ and $P_{s}^{\prime}$ are the transmit power of the primary and secondary users, respectively, $h\left(D^{\prime}\right)$ is the channel gain at distance $D^{\prime}$ from the transmitter and $P_{b}^{\prime}$ is the background interference power at the primary receiver.

To avoid causing harmful interference to the primary receiver, the secondary user must be able to detect a signal from the primary transmitter within the range of $D_{1}^{\prime}+D_{2}^{\prime}$ which can translate to a certain sensitivity requirement for the secondary detector. Although the cases where an active primary transmitter is present but it is far away from the secondary user fall under hypothesis $H_{1}$, the interference the primary receiver would not be harmful 
and as such these cases should be treated as white space by definition. However, since the detection of the primary transmitter is dependent on the SNR at the secondary user as seen in 2.1, it would be unlikely for low-SNR primary signals to trigger the secondary user's detector resulting in unusable white spaces [31].

\subsection{Sensing Techniques}

In this section, we will discuss some of the most common spectrum sensing techniques for the detection of the primary transmitter in the cognitive radio literature. From the perspective of signal detection, sensing techniques can be classified into two broad categories: coherent and non-coherent detection. In coherent detection, the primary signal can be coherently detected by comparing the received signal or the extracted signal characteristics with prior knowledge of primary signals. In non-coherent detection, no prior knowledge of the primary signal is required for detection. Another way to classify sensing techniques is based on the bandwidth of the spectrum of interest, that is, narrowband and wideband. The classification of sensing techniques is shown in Figure 2.3. Next, we introduce matched filter detection, energy detection, cyclostationary detection and briefly discuss some other spectrum sensing techniques. A more complete review on various spectrum sensing techniques and design challenges can be found in $[13,32]$.

\subsubsection{Matched Filter Detection}

Matched filtering is known as the optimum method for the detection of the primary signal when the transmitted signal is known, since it maximizes the received signal-to-noise ratio (SNR). The main advantage of matched filtering is the short time it requires to achieve a certain detection performance, such as low probabilities of miss-detection and false alarm [33], since a matched filter needs less received signal samples. However, matched filtering requires the secondary users to demodulate the received signals. Therefore, it requires perfect 


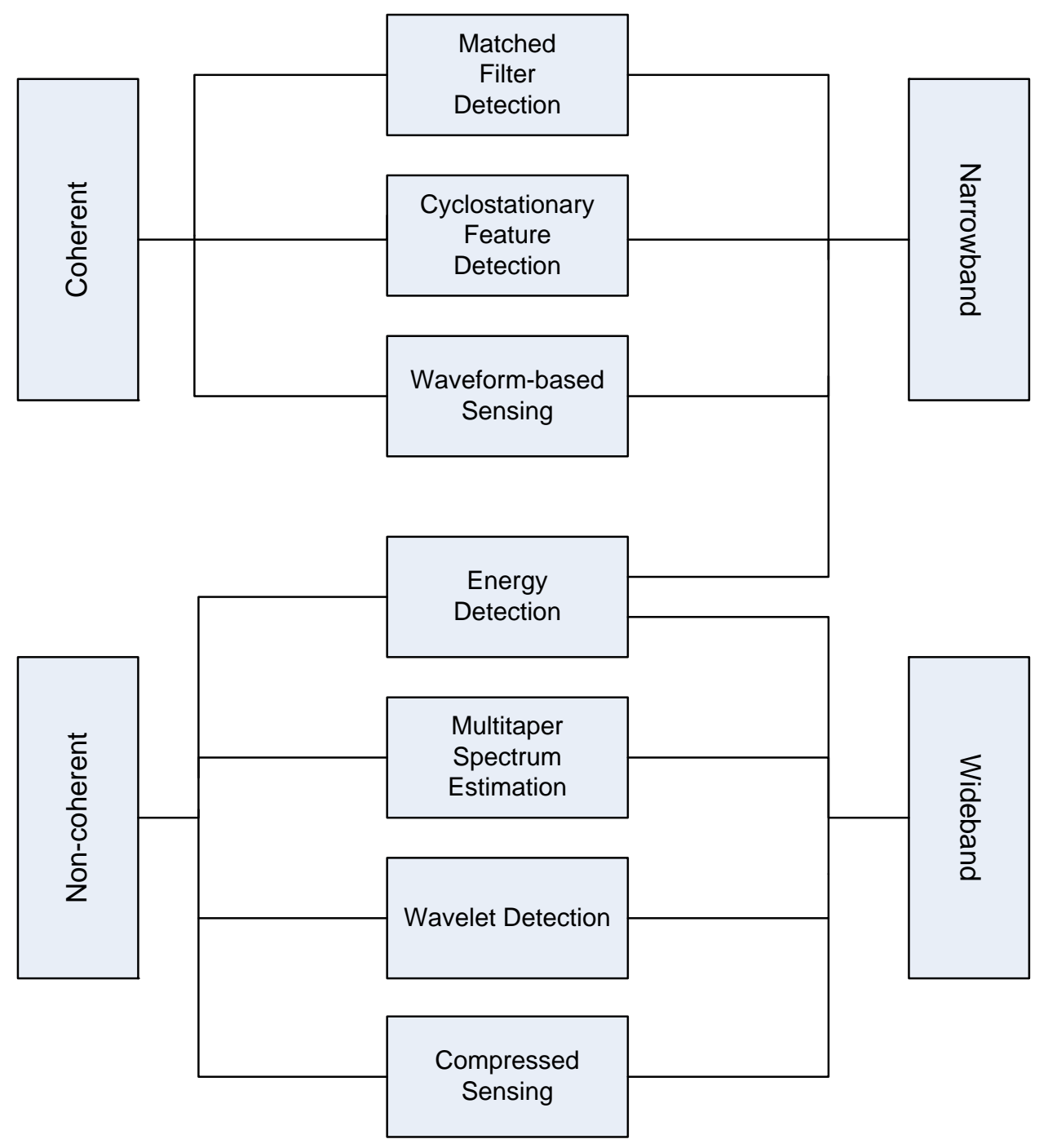

Figure 2.3: Classification of spectrum sensing techniques.

knowledge of the primary users signaling features such as bandwidth, operating frequency, modulation type and order, and pulse shaping as well as accurate synchronization at the secondary user $[12,14,32]$. However, in cognitive radio networks, such knowledge is not readily available to secondary users and the implementation cost and complexity of this detector are high. Another significant drawback of matched filter detection is that a secondary user would need a dedicated receiver for every primary user class [34]. 


\subsubsection{Energy Detection}

Energy detection $[24,35]$ is a non-coherent detection method that is most commonly used if the receiver cannot gather sufficient information about the primary user's signal. This simple scheme accumulates the energy of the received signal during the sensing interval and declares the primary band to be occupied if the energy surpasses a certain threshold which depends on the noise floor [24]. Due to its simplicity and the fact that it does not require prior knowledge of the primary users' signals, energy detection is the most popular sensing technique among others for spectrum sensing $[6,17,36-38]$. However, some of the challenges with energy detection include selection of the threshold for detecting primary users, inability to differentiate interference from primary users' transmission and noise, and poor performance under low signal-to-noise ratio [14]. Moreover, energy detection does not work efficiently for detecting spread spectrum signals for which more sophisticated signal processing algorithms need to be devised [39].

In addition to narrowband sensing, energy detection has been used for multiband joint detection in wideband sensing by employing an array of energy detectors, each of which detects one frequency band [40]. The multiband joint detection framework enables secondary users to simultaneously detect primary users' signals across multiple frequency bands for efficient management of the wideband spectrum resource at the cost of detection hardware.

\subsubsection{Cyclostationary Feature Detection}

Another detection method that can be applied for spectrum sensing is the cyclostationary feature detection. Modulated signals are in general coupled with sine wave carriers, pulse trains, repeating spreading or hopping sequences or cyclic prefixes, which result in builtin periodicity. Cyclostationary features are caused by the periodicity in the signal or in its statistics such as mean and autocorrelation [14]. Cyclostationary feature detection is a method for detecting primary user transmissions by exploiting the cyclostationary features of the received signals. Instead of power spectral density (PSD), cyclic correlation function is 
used for detecting signals present in a given spectrum. The cyclostationary-based detection algorithms can differentiate noise from primary users' signals. This is a result of the fact that noise is wide-sense stationary with no correlation while modulated signals are cyclostationary with spectral correlation due to the redundancy of signal periodicity. Therefore, a cyclostationary feature detector can perform better than the energy detector in discriminating against noise due to its robustness to the uncertainty in noise power [12,41]. However, it is computationally complex and requires significantly long observation time. Moreover, it requires the knowledge of the cyclic frequencies of the primary users, which may not be available to the secondary users.

\subsubsection{Other Sensing Techniques}

Alternative spectrum sensing methods include waveform-based sensing, multitaper spectral estimation, wavelet detection and compressed sensing. Waveform-based sensing is usually based on correlation with known signal patterns. Known patterns are usually utilized in wireless systems to assist synchronization or for other purposes. Such patterns include preambles, regularly transmitted pilot patterns and spreading sequences. In [39], it was shown that waveform-based sensing outperforms energy detector-based sensing in reliabil-

ity and convergence time. Furthermore, it is shown that the performance of the sensing algorithm increases as the length of the known signal pattern increases. Waveform-based sensing, however, is only possible when the target primary user's signal contains known signal patterns.

Multitaper spectrum estimation was proposed in [8]. The proposed algorithm was shown to be an approximation to the maximum likelihood power spectral density estimator, and for wideband signals, it is nearly optimal. Most important, unlike the maximum-likelihood spectral estimator, the multitaper spectral estimator is computationally feasible. In [42], wavelets are used for detecting edges in the power spectral density of a wideband channel. Once the edges, which correspond to transitions from an occupied band to an empty band or 
Table 2.1: Comparison of Spectrum Sensing Techniques.

\begin{tabular}{|l|l|l|}
\hline $\begin{array}{l}\text { Spectrum sensing tech- } \\
\text { nique }\end{array}$ & Advantages & Disadvantages \\
\hline Matched filter detection & $\begin{array}{l}\text { - Optimal performance } \\
\text { - Low computational cost }\end{array}$ & $\begin{array}{l}\text { Requires prior knowledge of } \\
\text { the primary user's signal }\end{array}$ \\
\hline Energy detection & $\begin{array}{l}\text { - Low complexity } \\
\text { - No primary knowledge re- } \\
\text { quired }\end{array}$ & $\begin{array}{l}\text { - Poor performance for low } \\
\text { SNR } \\
\text { - Cannot differentiate users }\end{array}$ \\
\hline Cyclostationary detection & $\begin{array}{l}\text { - Robust in low SNR region } \\
\text { - Robust against interfer- } \\
\text { ence }\end{array}$ & $\begin{array}{l}\text { - Requires partial prior in- } \\
\text { formation } \\
\text { - High computational cost }\end{array}$ \\
\hline Waveform-based detection & $\begin{array}{l}\text { - Robust in low SNR region } \\
\text { - Short measuring time }\end{array}$ & $\begin{array}{l}\text { - Requires prior knowledge } \\
\text { of the primary user's signal } \\
\text { - Susceptible to synchroniza- } \\
\text { tion errors }\end{array}$ \\
\hline $\begin{array}{l}\text { Multitaper spectrum esti- } \\
\text { mation }\end{array}$ & $\begin{array}{l}\text { - Near optimal performance } \\
\text { for wideband signals } \\
\text { - No primary knowledge re- } \\
\text { quired }\end{array}$ & $\begin{array}{l}\text { High implementation com- } \\
\text { plexity }\end{array}$ \\
\hline Wavelet Detection & $\begin{array}{l}\text { Effective for wideband sig- } \\
\text { nal detection }\end{array}$ & $\begin{array}{l}\text { - Requires high sampling } \\
\text { rate analog-to-digital con- } \\
\text { verter } \\
\text { - High computational cost }\end{array}$ \\
\hline Compressed sensing & $\begin{array}{l}\text { - Low sampling rate } \\
\text { - Low signal acquisition cost }\end{array}$ & $\begin{array}{l}\text { Sensitive to design imperfec- } \\
\text { tions }\end{array}$ \\
\hline
\end{tabular}

vice versa, are detected, the power within the bands between two edges are estimated. Using this information and the edges positions, the power spectral density can be characterized as occupied or empty in a binary fashion. The assumptions made in [42], however, need to be relaxed for building a practical sensing algorithm. The method proposed in [42] was extended in [43] by using sub-Nyquist sampling (compressed sensing). Assuming that the signal spectrum is sparse, sub-Nyquist sampling is used to obtain a coarse spectrum knowledge in an efficient way. Table 2.1 presents a brief comparison of the above spectrum sensing techniques. 
In this thesis, we adopt the most commonly used energy detection technique for the detection of the primary transmitter. With this technique, secondary users can identify the spectrum access opportunities without requiring prior knowledge of the primary users' signal characteristics. Compared to other sensing techniques, energy detection is easier to implement and therefore, is less expensive. It is also the most general technique for spectrum sensing since it applies to any signal type.

\subsection{Cooperative Spectrum Sensing (CSS)}

In cooperative spectrum sensing, information from multiple secondary users are incorporated for the detection of the primary signal. In the literature, cooperation is discussed as a solution to problems that arise in spectrum sensing due to noise uncertainty, fading, and shadowing since the uncertainty in a single user's detection can be minimized [36]. The main idea of cooperative sensing is to enhance the sensing performance by exploiting the spatial diversity in the observations of spatially located secondary users. By cooperation, secondary users can share their sensing information for making a combined decision more accurate than the individual decisions [19]. The performance improvement due to spatial

diversity is called cooperative gain. While cooperative gain such as improved detection performance and relaxed sensitivity requirement can be obtained, cooperative sensing can incur cooperation overhead. Cooperation overhead refers to any extra sensing time, delay, energy, and operations devoted to cooperative sensing and any performance degradation caused by cooperative sensing.

\subsubsection{Cooperation Architecture}

Depending on how the secondary users share their sensing data, several cooperative spectrum sensing architectures for CR networks have been proposed in the literature $[17,44-46]$. The most commonly proposed architecture is the parallel fusion architecture, in which all the 
sensing secondary users send their sensing information directly to a centralized controller called a fusion center. This fusion center then makes a final decision regarding the presence or absence of the primary signal, and broadcasts this information to other secondary users or directly controls the cognitive radio network traffic $[17,36,44]$. The parallel fusion architecture is illustrated in Figure 2.4.

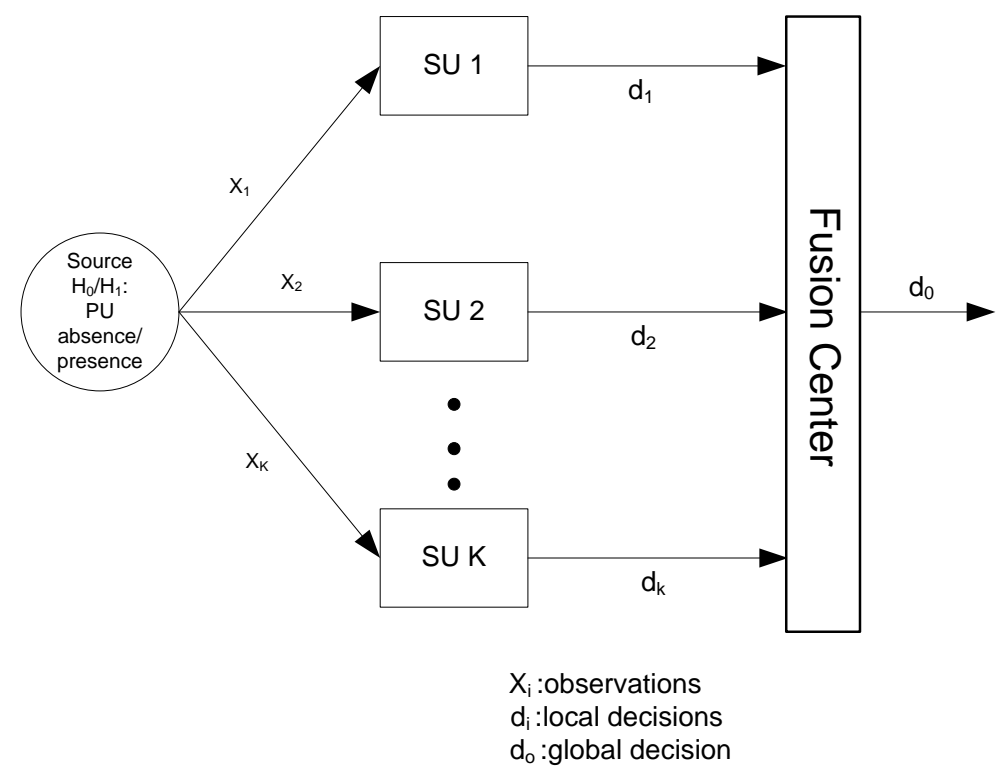

Figure 2.4: Parallel fusion architecture.

Another possible sensing architecture is the decentralized sensing architecture which does not rely on a fusion center for making the cooperative decision $[18,39,46]$. In this case, secondary users exchange the sensing observations and converge to a unified decision on the presence or absence of primary users transmissions by iterations. Based on a distributed algorithm, each secondary user sends its own sensing data to other users, combines its data with the received sensing data, and decides whether or not the primary user's transmission is present by using a local criterion. If the criterion is not satisfied, secondary users send their combined results to other users again, and repeat this process until the algorithm is converged and a decision is reached. The decentralized sensing architecture is illustrated in Figure 2.5. 


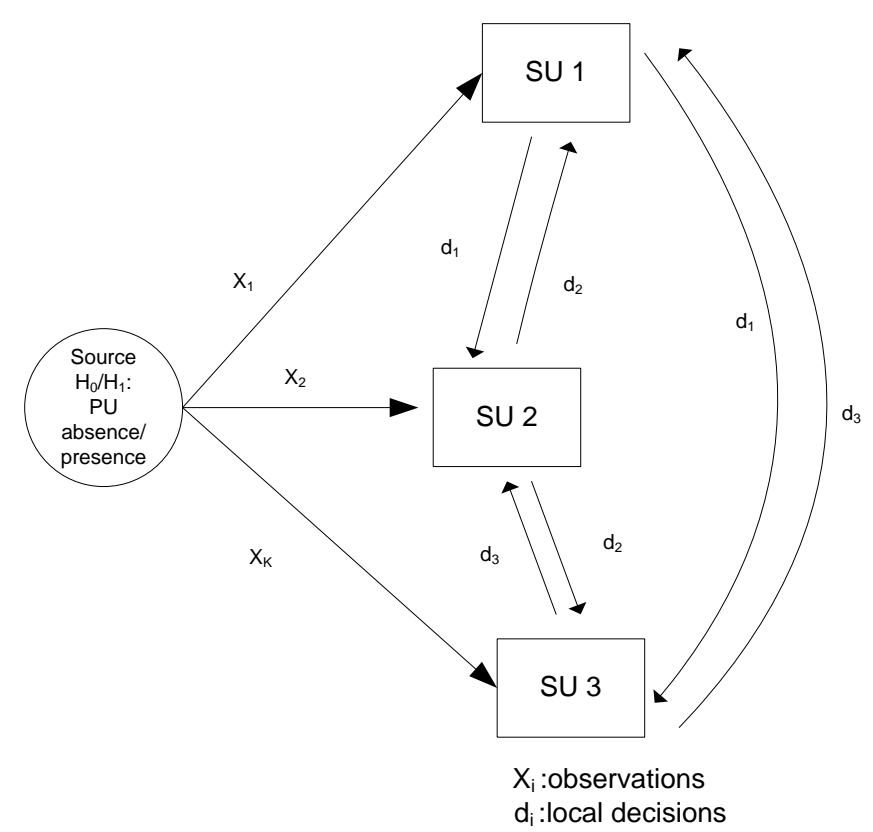

Figure 2.5: Decentralized fusion architecture.

\subsubsection{Fusion Schemes}

In cooperative sensing, a fusion scheme refers to the process of combining locally sensed data of individual secondary users. Depending on which type of sensing data is transmitted to the fusion center or shared with neighboring users, CSS can employ data or decision fusion schemes. In soft decision schemes (data fusion), secondary users exchange their test statistics calculated from their local observations. On the other hand, in the hard decision schemes (decision fusion), secondary users only exchange their individual binary decisions.

\section{Soft Combining and Data Fusion}

Existing receiver diversity techniques such as equal gain combining (EGC) and maximal ratio combining (MRC) can be utilized for soft combining of local observations or test statistics. If the channel state information (CSI) between the primary users and the secondary users are perfectly known, the optimal combining strategy, which is MRC, can be used for achieving the highest output SNR. It was shown in [25] that the soft combining scheme yields better gain than the hard combining scheme. However, there is a significant differ- 
ence in the cooperation overhead between the hard and soft decision based detectors, which requires a wideband control channel for the soft decision cooperative approach. The soft information based signal detection method for the single-carrier case and multi-carrier case was investigated in [47]. In [37], a linear cooperation strategy was developed which is based on the optimal combination of the local statistics from spatially distributed secondary users.

In [48], an optimal soft combination scheme based on Neyman-Pearson criterion was proposed to combine the weighted local observations. The proposed scheme reduces to EGC at high SNR and reduces to MRC at low SNR. Since such a soft combining scheme results in large overhead, a softened two-bit combining scheme was also proposed in [48] for energy detection. In this method, there are three decision thresholds dividing the whole range of test statistics into four regions. Each secondary user reports the quantized two-bit information of its local test statistics. The performance of this method is comparable to the performance of the EGC scheme with less complexity and overhead.

\section{Hard Combining and Decision Fusion}

In the hard combining scheme, the final decision is reached by taking into consideration the individual local decisions reported by each secondary user. When binary local decisions are reported to the fusion center, it is convenient to apply linear fusion rules to obtain the cooperative decision. The main advantage of the hard combining scheme is the reduction of communication overhead. Hard decision combining for CSS has been considered in several works $[25,48,49]$. The commonly used fusion rules are AND, OR, and majority voting rules which are special cases of the general $K$-out-of- $M$ rule. Those decision fusion rules can be summarized as below [50]:

- $K$-out-of- $M$ rule: In this fusion rule, the fusion center decides on the presence of the primary user's transmission if, and only if, $K$ or more than $K$ secondary users out of the total $M$ cooperating secondary users report the detection of the primary user's signal, where $K \in[1, M]$. Therefore, in the $K$-out-of- $M$ rule, if $K$ users or more decide 
in favor of $H_{1}$, then the cooperative decision declares that $H_{1}$ is true. If the decisions from all the secondary users are independent, the network probabilities of detection and false alarm are, respectively, given by [51]:

$$
P_{D}=\sum_{k=0}^{M-K}\left(\begin{array}{c}
M \\
K+k
\end{array}\right)\left(1-P_{d, k}\right)^{M-K-k}\left(P_{d, k}\right)^{K+k}
$$

and

$$
P_{F}=\sum_{k=0}^{M-K}\left(\begin{array}{c}
M \\
K+k
\end{array}\right)\left(1-P_{f, k}\right)^{M-K-k}\left(P_{f, k}\right)^{K+k}
$$

where $P_{d, k}$ and $P_{f, k}$ are, respectively, the probabilities of detection and false alarm of the $k^{\text {th }}$ secondary user and $\left(\begin{array}{c}M \\ K+k\end{array}\right)=\frac{M !}{(K+k) !(M-K-k) !}$.

- Majority voting (MV) rule: In the MV fusion rule, also known as half voting rule, if half, or more than half, the local detectors decide that there is a primary user's transmission, then the final decision at the fusion center declares that there is a primary user's transmission [50]. Therefore, for the MV rule, the cooperative decision declares $H_{1}$ only if half or more than half of the secondary users decide on $H_{1}$, i.e., $K=\left\lceil\frac{M}{2}\right\rceil$ in $(2.10)$ and $(2.11)$, where $\left\lceil\frac{M}{2}\right\rceil$ denotes the smallest integer not less than $\frac{M}{2}$. If the decisions from all the secondary users are independent, the network probabilities of detection and false alarm are, respectively, given by

$$
P_{D}=\sum_{k=0}^{M-\left\lceil\frac{M}{2}\right\rceil}\left(\begin{array}{c}
M \\
\left\lceil\frac{M}{2}\right\rceil+k
\end{array}\right)\left(1-P_{d, k}\right)^{M-\left\lceil\frac{M}{2}\right\rceil-k}\left(P_{d, k}\right)^{\left\lceil\frac{M}{2}\right\rceil+k}
$$

and

$$
P_{F}=\sum_{k=0}^{M-\left\lceil\frac{M}{2}\right\rceil}\left(\begin{array}{c}
M \\
\left\lceil\frac{M}{2}\right\rceil+k
\end{array}\right)\left(1-P_{f, k}\right)^{M-\left\lceil\frac{M}{2}\right\rceil-k}\left(P_{f, k}\right)^{\left\lceil\frac{M}{2}\right\rceil+k}
$$

- Logical OR rule: In this fusion rule, the fusion decides on the presence of primary user's transmission if any of the secondary users reports the detection of the primary user's transmission. Therefore, for the OR rule, the cooperative decision declares $H_{1}$ if 
any of the secondary users decides on $H_{1}$, i.e., setting $K=1$ in (2.10) and (2.11). Since a secondary user (SU) occupying a licensed frequency band may cause interference to the primary users, the risk of SUs causing interference to the primary users is minimized using the logical OR rule. If the decisions from all the secondary users are independent, the network probabilities of detection and false alarm are, respectively, given by

$$
P_{D}=1-\prod_{k=1}^{M}\left(1-P_{d, k}\right)
$$

and

$$
P_{F}=1-\prod_{k=1}^{M}\left(1-P_{f, k}\right) .
$$

- Logical AND rule: In the AND fusion rule, if all local detectors decide that there is a primary user's transmission, then the final decision at the fusion center declares that there is a primary user's transmission [50]. Therefore, for the AND rule, the cooperative decision declares $H_{1}$ only if all of the secondary users decide on $H_{1}$, i.e., setting $K=M$ in (2.10) and (2.11). Using this fusion rule, the probability of false alarm is minimized, but the risk of causing interference to primary users will increase. If the decisions from all the secondary users are independent, the network probabilities of detection and false alarm are, respectively, given by

$$
P_{D}=\prod_{k=1}^{M} P_{d, k}
$$

and

$$
P_{F}=\prod_{k=1}^{M} P_{f, k} .
$$

\subsubsection{Cooperative User Selection}

The selection of secondary users for cooperative sensing plays a key role in determining the performance of CSS because it can be utilized to improve the trade-off between cooperative 
gain and cooperation overhead. In [49], for the case of independent secondary users' observations with energy detection based cooperation, it was shown that cooperating with all users in the network does not necessarily achieve the optimum performance. It was observed that including secondary users experiencing bad channels, in terms of the SNR received at a secondary user, for cooperation may degrade the performance. In order to relax the requirement on prior knowledge of the received SNR at each secondary user, the authors in [52] proposed to select the sensing secondary users that have the best detection probabilities with respect to a given false alarm probability. Specifically, the false alarm probability is set to be identical at each secondary user. Therefore, the SU that reports the largest number of 1's is first chosen to participate in cooperative sensing. In [53], the optimal number of secondary users, $K$, that minimizes the total error probability for secondary users with independent local decisions for the general $K$-out-of- $M$ fusion rule was found to be approximately half of the total number of secondary users $M$. A user selection strategy based on a modified deflection coefficient with low complexity was proposed in [54]. The optimal number of secondary users and the user set were obtained in order to provide sufficient protection to the primary users and improve the total throughput of the cognitive radio network.

When cooperating secondary users experience correlated shadowing, it was shown in [20] that selecting independent secondary users for cooperation can improve the robustness of sensing results. In [55], a distributed user selection algorithm was developed to address the dynamic changes in the spatial correlation experienced by mobile secondary users and adaptively select uncorrelated secondary users.

Moreover, removing malicious users from cooperation ensures the security and the reliability of the network. A robust secondary user selection algorithm for CSS considering the presence of malicious users was proposed in [56]. The users were selected based on the consistency check with known trusted users and simulation results showed that the proposed algorithm is effective in identifying and excluding malicious secondary users. The authors in [57] presented a soft-decision reporting scheme that is robust against malicious users. 
They proposed a heuristic approach to iteratively identify malicious users, where the fusion center computes the secondary user's suspicious level, i.e., the posterior probability that this $\mathrm{SU}$ is an attacker, based on the honest SUs and malicious SUs report probabilities. These probabilities are estimated assuming that the fusion center knows the position of the users and the attackers' policy. When the suspicious level of the secondary user goes beyond a threshold it is discarded from the final decision process and moved into a malicious user set. This process is repeated until no more malicious users can be found and only the reports from honest users are fused to make the final decision.

\subsubsection{Cooperation Overhead}

The exploitation of spatial diversity in cooperative sensing results in a significant improvement in detection performance. However, cooperation among secondary users may also introduce a variety of overheads that limit or even compromise this improved detection performance. The overhead associated with all elements of cooperative sensing is called cooperation overhead. Cooperation overhead can refer to any transmission cost, extra sensing time, delay, energy and operations devoted to cooperative sensing and any performance degradation caused by cooperative sensing.

Since the sensing time is proportional to the number of samples taken by each individual secondary user, the longer the sensing time is, the better the detection performance will be. However, when each secondary user is equipped with a single radio transceiver, it will be difficult for the secondary users to simultaneously perform sensing and transmission. Therefore, the more time is devoted to sensing, the less time is available for transmissions which reduces the secondary users' throughput, also known as opportunistic throughput. In addition, the cooperation overhead due to the extra sensing time will generally increase with the number of cooperating users due to the increased volume of data that needs to be reported to and be processed by the fusion center. This is known as the sensing efficiency problem [58] or the sensing-throughput trade-off [51] in spectrum sensing. 
The cooperation overhead, in terms of the extra sensing time or reduced opportunistic throughput, will also increase as the delay in finding an available channel increases [59]. In [60], a sensing-period optimization mechanism and an optimal channel-sequencing algorithm were developed to maximize the discovery of spectrum access opportunities and minimize the delay in discovering an available channel when all secondary users participate in sensing an identical channel in each sensing period. Parallel cooperative sensing was proposed in $[61,62]$ where the cooperative secondary users are divided into multiple groups and each group senses one channel such that more than one channel is sensed in each sensing period. Since multiple channels are detected in one sensing period, the cooperation overhead associated with the delay in finding an available channel is significantly reduced.

In cooperative sensing, secondary users involve in activities such as local sensing and data reporting that consume additional energy. The energy consumption overhead can be significant if the number of cooperating secondary users or the amount of sensing results to be reported is large. One approach to address this issue is to use censoring to limit the amount of reported sensing data according to certain criteria or constraints. Since the censoring criteria are chosen to refrain cooperating secondary users from transmitting unnecessary or un-informative data, the energy efficiency can be improved in cooperative sensing. In [63], a simple censoring method was proposed to decrease the average number of sensing bits reported to the fusion center. In this method, the energy detector output of each secondary user is compared to two thresholds and the decision is sent to the fusion center if the energy detector output is between those two thresholds. Otherwise, no decision is made and this sensing output is censored from reporting. The results showed that even though the network probability of false alarm may degrade due to the possibility that the sensing outputs of all secondary users are censored, the amount of reported local decisions can be dramatically reduced. Therefore, the energy efficiency can be traded off with the network probability of false alarm.

Another approach to reduce the cooperation overhead in terms of energy consumption 
is to minimize the energy consumption with detection performance constraints. In [64], the energy efficiency problem was addressed by energy minimization under detection performance constraints. This method investigates the trade-off between the two aspects of sensing time. On one hand, longer sensing time consumes more energy at each secondary user. On the other hand, longer sensing time can improve detection performance at each secondary user and reduce the number of cooperating users and the associated energy consumption overhead. Therefore, this method finds the optimal sensing time and the optimal number of cooperating users to balance the energy consumption in local sensing and the energy overhead due to cooperation for a required detection performance.

\subsubsection{Sensing Errors}

A secondary user identifies spectrum access opportunities by detecting the presence of primary signals. Sensing errors, in terms of false alarms or miss-detections, occur due to noise and fading. False alarms occur when idle channels are detected as busy, and miss-detections occur when busy channels are detected as idle. In the event of a false alarm, a spectrum access opportunity is overlooked by the secondary user, and eventually wasted if the access strategy trusts the sensing outcome. On the other hand, miss-detections may lead to collisions with primary users' transmissions. Therefore, in spectrum sensing, it is desired to minimize the probability of sensing error (i.e., sum of the probability of false alarm and the probability of miss-detection) which reduces the collision probability with primary users' transmissions and enhances the usage level of vacant spectrum. A well chosen detection threshold can minimize spectrum sensing errors, provide the primary users' transmissions with enough protection, and fully enhance spectrum utilization. In [53], the optimal threshold level for minimizing the probability of sensing error was determined without considering spectrum sensing constraints that may be violated. To alleviate this problem, an adaptive

optimal spectrum sensing threshold level was derived in [65] to minimize the probability of sensing error while satisfying spectrum sensing constraints on the probabilities of false alarm 
and miss-detection. CSS using counting rule was studied in [66] and the sensing errors were minimized by choosing the optimal probability of false alarm to satisfy a given constraint and the optimal number of cooperating secondary users for both matched filtering and energy detection. CSS with correlated secondary users' local decisions was studied in [67]. The probability of sensing error was minimized by choosing the optimal assignments for the number of cooperating secondary users, $K$, in the $K$-out-of- $M$ fusion rule and the local threshold for a certain correlation index.

It is usually assumed that the local observations and the combining decision are all made at the same time. In reality, this is not always valid and therefore, the CSS scheme should consider the case of asynchronous observations which results in time offsets between local sensing observations and the final decision at the fusion center. In [68], a probability-based combination scheme was proposed to combine asynchronous reports at the fusion center. The proposed combining scheme considers both detection errors and time offsets between local sensing observations and the final decision. Based on the knowledge of the primary user channel usage model and the Bayesian decision rule, the conditional probabilities of the local sensing decisions received at different times, conditioned on each hypothesis, and their combined likelihood ratio were calculated to make the final decision at the fusion center.

Most of the studies on CSS analyze its performance based on the assumption of perfect knowledge of the average received SNR at the secondary user. However, in practice, this is not always the case. The effect of average SNR estimation errors on the performance of CSS was examined in [69]. In the noiseless-sample-based case, it was found that the probability of false alarm decreases as the average SNR estimation error decreases for both independent and correlated shadowing. In the noise-sample-based case, it was found that there exists a threshold for the noise level. Below this threshold, the probability of false alarm increases as the noise level increases, while above the threshold the probability of false alarm decreases as the noise level increases. 


\subsubsection{Multiband Spectrum Sensing}

Wideband spectrum sensing, that we also refer to in this thesis as multiband sensing, faces technical challenges and there is limited work on it in the literature. To sense multiple frequency bands, secondary users may need to scan the spectrum or use multiple radio frequency $(\mathrm{RF})$ front ends for sensing multiple bands. However, using these approaches for wideband sensing either causes long sensing delay or incurs high computational complexity and hardware cost. Recent advances in compressed sensing $[43,70]$ enable the sampling of the wideband signals at sub-Nyquist rate to relax the analog-to-digital converter (ADC) requirements. The techniques of compressed sensing provide promising solutions to promptly recover wideband signals and facilitate wideband sensing at a reasonable computational complexity. Compressed sensing is achieved by the random sampling of wideband signals. However, due to the sub-Nyquist-rate sampling and insufficient number of samples, a weak primary user's signal with a nearby strong signal may not be properly reconstructed for detection in a wideband spectrum [19]. In addition, a new ADC architecture with nonuniform timing and a pseudo-random clock generator is needed [71].

In multiband cooperative sensing, secondary users cooperate to sense multiple narrow bands instead of focusing on one band at a time. In [40], a multiband joint detection scheme was proposed for combining the statistics of sensing multiple bands from spatially distributed secondary users. The fusion center calculates the test statistic and makes a cooperative decision in each band. The weight coefficients and detection thresholds of all bands were obtained by jointly maximizing the aggregate opportunistic throughput in each band subject to constraints on the miss-detection and false alarm probabilities. To enable the multiband sensing at each secondary user, an energy detector is required for each band of interest. As a result, the method may incur high hardware cost when the number of bands for cooperative sensing is large. In [72], the authors proposed a multiband adaptive joint detection framework for wideband spectrum sensing that collectively searches the secondary transmission opportunities over multiple frequency bands. In this framework, both the 
sensing slot duration and detection thresholds for each narrowband detector were jointly optimized to maximize the achievable opportunistic throughput of the secondary network subject to a limit on the interference introduced to primary users.

In [61], a parallel cooperative sensing scheme was proposed to enable the multi-channel sensing by optimally selected cooperating secondary users. Different from the multiband sensing scheme in [40,72], each cooperating secondary user senses a different channel. In [73], the authors proposed a group-based CSS scheme in which the cooperative secondary users are divided into several groups and each group senses a different channel during a sensing period while the secondary users in the same group perform joint detection on the targeted channel. By the methods in $[61,73]$, multiple channels can be cooperatively sensed in each sensing period. The objective is to maximize the secondary opportunistic throughput while minimizing the sensing overhead such as the sensing time and the number of secondary users required for cooperation.

In this thesis, we focus on multi-channel sensing by cooperating secondary users in which more than one channel can be sensed in each sensing period to leverage the cooperative gain of CSS. To this end, we study both the group-based CSS and the multiband cooperative joint detection framework. Throughout this work, we employ one-bit hard decision combining-based CSS since it is more applicable to practical system due to the reduced feedback signalling overhead as compared to the soft decision combining-based CSS.

\subsection{Chapter Summary}

In this chapter, we presented the basic concepts of spectrum sensing which is one of the fundamental prerequisites for the successful deployment of cognitive radio networks. We reviewed the most common spectrum sensing techniques with which the secondary users are able to monitor the activities of the primary users. Various spectrum sensing techniques are used in the literature depending on how much information about the primary signal is 
available to the secondary users. In general, the spectrum sensing techniques can be classified as energy-based sensing, cyclostationary feature-based sensing, matched filter-based sensing and other sensing techniques. Energy detection is the most commonly used technique for spectrum sensing since it has low computational and implementation complexities and prior knowledge of the primary users' signal is not needed.

The unreliability in individual secondary user's sensing results gives rise to sensing errors which affects the sensing accuracy. To increase the sensing accuracy, performance metrics such as the probabilities of detection and false alarm and the probability of sensing error should be considered in the design of spectrum sensing techniques. To address the limitations of the spectrum sensing techniques by a single secondary user, cooperative spectrum sensing and its main elements have been discussed. Cooperative sensing is an effective technique to improve sensing accuracy by exploring multiuser diversity at the expense of cooperation overhead in terms of increased sensing time, energy consumption or reduced opportunistic throughput.

In addition to sensing accuracy, sensing efficiency, which can be represented by the metrics of sensing overhead and secondary throughput, has a significant impact on the performance of spectrum sensing. However, there is a fundamental trade-off between sensing accuracy and sensing efficiency in spectrum sensing. To increase the sensing efficiency, multiband joint detection and parallel cooperative sensing can be employed to discover more spectrum access opportunities in a single sensing period.

In the next chapter, we address the problem of sensing accuracy-efficiency trade-off. We consider a group-based cooperative spectrum sensing scheme where the cooperative secondary users are divided into multiple groups while each group senses one channel in each sensing period. We design an efficient assignment algorithm for both the users and groups that addresses the problem of sensing accuracy-efficiency trade-off in group-based cooperative spectrum sensing. 


\section{Chapter 3}

\section{Adaptive User Assignment for Group-based Cooperative Spectrum Sensing}

\subsection{Introduction}

As discussed in Chapter 2, spectrum sensing must be able to detect the primary user's signal levels accurately so that the primary user's transmission is adequately protected and the number of false alarms is minimized. In this chapter, we consider the energy detection approach since it has low computational and implementation complexities, and prior knowledge of the primary users' signal is not needed [14]. The secondary users employ a periodic sensing-transmission structure in which the sensing and transmission are performed periodically in separate periods $[15,21]$.

Spectrum sensing performance can be improved using network cooperation where secondary users share their spectrum sensing measurements $[74,75]$. To increase the cooperative gain (i.e., sensing accuracy), more cooperating secondary users should perform the sensing. However, using more cooperating users will lead to an increase in the amount of overhead 
traffic in the secondary network [76]. In the conventional sequential spectrum sensing, all cooperative secondary users sense the same channel in each sensing period to increase the sensing accuracy. Since only one channel could be sensed by the cooperating secondary users during each sensing period, this leads to a delay in the discovery of available channels which degrades the sensing efficiency.

In this chapter, we consider a group-based cooperative spectrum sensing (CSS) scheme for secondary users with heterogeneous sensing ability in terms of sensing accuracy. The sensing accuracy is represented by the probabilities of detection and false alarm which are different for different secondary users. In group-based CSS, each group senses a different channel while the secondary users in the same group cooperate together to sense the targeted channel. By employing this CSS scheme, more than one channel can be sensed in each sensing period which can significantly improve the sensing efficiency. For group-based cooperative spectrum sensing, we propose to adaptively assign the heterogeneous cooperating secondary users to different groups to achieve a trade-off between the sensing efficiency and sensing accuracy. Our aim is to maximize the throughput efficiency without degrading the sensing accuracy. To this end, we analytically derive the throughput efficiency, which we define in terms of the average opportunistic throughput and average sensing overhead. We then formulate the throughput efficiency maximization problem for heterogeneous secondary users as a non-linear binary programming problem which is computationally intractable. To solve the throughput efficiency maximization problem, we propose an efficient adaptive assignment algorithm that assigns users to groups and then assigns those groups to the sensing rounds such that the throughput efficiency is maximized.

\subsection{Related Work}

Enhancing the sensing accuracy of CSS was extensively treated in $[25,36,40,58,77,78]$. In $[25,36]$, the AND and OR fusion rules were studied under the Neyman-Pearson criterion, 
when the sensing objective was to maximize the probability of detection under a constraint on the probability of false alarm. The authors of [77] considered cooperative sensing by using a counting rule and calculated the optimal number of cooperating secondary users and optimal probability of false alarm for each user under both the Neyman-Pearson criterion and the Bayesian criterion. In [58], the authors optimized the sensing parameters by maximizing the transmission time with respect to the total frame period (transmission time and sensing time) subject to interference avoidance constraints in a single spectrum band. The authors also introduced spectrum selection and scheduling methods where the best spectrum bands for sensing were selected to maximize the opportunistic sensing capacity which represents the expected transmission capacity that the secondary users can achieve on the selected bands.

In [78], the sensing time setting for a multi-user multi-channel case with CSS was investigated. In this CSS approach, secondary users cooperatively sense the channels and send the sensing results to a coordinator, which employs energy detection with a soft decision rule to estimate whether there are primary activities in the sensed channels. The sensing time setting determines the length of the sensing time in a slot and how the total sensing time (of the multiple users) in a slot is distributed among all the channels to increase the sensing accuracy. This is done by maximizing the opportunistic throughput of the secondary users on all the channels subject to a limit on the probabilities of detection and false alarm for each channel. However, the authors did not consider the effect of cooperation overhead on the performance of their CSS approach which is expected to be large due to the assumption that a secondary user can send its test statistic directly to the fusion center. The study in [40] presented a new cooperative wideband spectrum sensing scheme that exploits the spatial diversity among multiple SUs. The weight coefficients for each user and detection thresholds of all bands were obtained by jointly maximizing the aggregate opportunistic throughput in each band subject to constraints on miss-detection and false alarm probabilities, which contribute to improving the sensing accuracy. The above studies have mainly focused on improving the sensing accuracy while the sensing efficiency has been ignored. 
The enhancement of sensing efficiency was investigated in $[60-62,79,80]$. In [60], a sensing-period optimization mechanism and an optimal channel-sequencing algorithm were developed to maximize the discovery of spectrum access opportunities and minimize the delay in discovering an available channel when all secondary users participate in sensing a channel simultaneously. In [79], two different channel sensing policies, the random sensing policy and the negotiation-based sensing policy, were proposed to discover the available channels. To improve the sensing efficiency, different users are allowed to sense different channels, that are selected either randomly or through negotiation, by assuming that each SU is equipped with two transceivers. The first transceiver (called the control transceiver) is devoted to operating over a dedicated control channel to obtain the information of the vacant licensed channels, and to negotiate with the other SUs when contention-based protocols are employed. The second transceiver is used to sense the licensed channels and to receive/transmit the SUs' packets. To reduce the sensing overhead, the authors of [80] proposed a multi-channel cooperative sensing scheme, where the cooperative SUs are optimally selected to sense the distinct channels at the same time for sensing efficiency. These works assume that the sensing accuracy on a channel sensed by a single SU is completely correct which may not be practical in real communication systems.

The authors in [61] proposed full parallel cooperative spectrum sensing, where each user senses a distinct channel, with the aim of discovering multiple spectrum access opportunities in a single sensing period. To enhance the spectrum efficiency, the authors optimized the number of sensing SUs and adaptively changed the stopping threshold in searching for available channels by considering the wireless channel dynamics. In [62], the authors proposed a group-based cooperative spectrum sensing scheme in which the cooperative SUs are divided into several groups and each group senses a different channel during a sensing period while SUs in the same group perform joint detection on the targeted channel. The sensing process will not stop unless an available channel is discovered. Assuming that all secondary users have equal probabilities of detection and false alarm, they formulated the achievable 
throughput maximization problem to determine the number of groups and number of users in each group in time varying channels.

The most relevant to our work is [62]. It was shown there that by varying the number of groups and the number of cooperating SUs in each group, the trade-off between sensing accuracy and efficiency could be adjusted. However, similar to most existing work on cooperative spectrum sensing, it was assumed that all the secondary users have the same energy detection threshold and identical average signal-to-noise ratio (SNR) to the primary user [81]. In reality, however, the average SNR will vary since secondary users are at different distances from the primary user. Therefore, in contrast to the work in [62], we consider that the cooperating secondary users have heterogeneous sensing ability in terms of the sensing accuracy which will be represented by non-identical local probabilities of detection and false alarm. This will introduce new challenges to the group-based cooperative spectrum sensing. The cooperating heterogeneous secondary users have to be adaptively assigned to different groups in order to achieve a trade-off between the sensing efficiency and sensing accuracy. To this end, we analytically derive an expression for the average opportunistic throughput and average sensing overhead for the group-based CSS scheme when the secondary users have heterogeneous sensing ability while incorporating the cost of using multiple sensing rounds to find a vacant channel which was not considered in [62]. In the next section, we describe the system model used in our analysis.

\subsection{System Model}

We consider a cognitive radio network with $K$ secondary users, $U^{\prime}$ primary users and $L$ licensed channels. We assume that all the considered secondary users utilize the licensed channels used by the same set of primary users. Therefore, the licensed channel availability information sensed by each secondary user is consistent among all secondary users [79]. An illustration of this cognitive radio network is shown in Figure 3.1. Considering that there is 


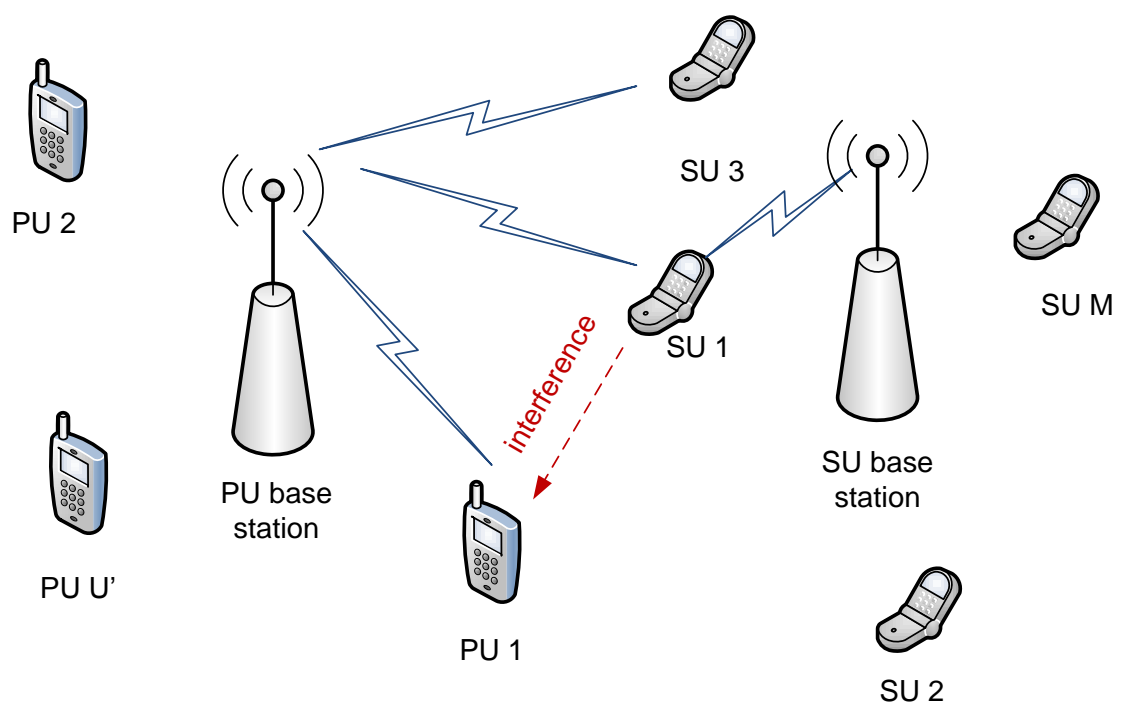

Figure 3.1: An illustration of a cognitive radio network.

no information exchange between PUs and SUs, each SU needs to perform spectrum sensing independently. We employ the computationally efficient and widely used energy detection technique $[24,35]$ for spectrum sensing. Energy detection requires no information about the primary user's signal which is more practical. In addition, we model the primary signal as a random variable with known transmit power and therefore, energy detection is optimal [44].

\subsubsection{Energy Detection}

The objective of spectrum sensing is to detect the presence of primary signals on the primary channel, and we model this problem as a binary hypothesis testing problem and use the energy detection technique for spectrum sensing. The block diagram for the energy detection technique is shown in Figure 3.2.

In the energy detection model, the problem of detecting the presence of primary users is equivalent to distinguishing between the two following hypotheses [19],

$$
x_{k}(n)= \begin{cases}v_{k}(n), & H_{0} \\ h_{k} s(n)+v_{k}(n), & H_{1}\end{cases}
$$


where $x_{k}(n)$ is the received signal of the $k^{t h}$ secondary user at the $n^{\text {th }}$ time instant, $h_{k}$ is the $k^{\text {th }}$ user channel gain which is assumed to be constant during the detection interval. The primary user's transmitted signal, $s(n)$, is an independent identically distributed (i.i.d.) random process with mean zero and variance $\sigma_{s}^{2}$ and is assumed to be BPSK modulated. The noise, $v_{k}(n)$, is a real-valued Gaussian variable with zero mean and variance $\sigma_{v}^{2}$. Without loss of generality, $s(n)$ and $v_{k}(n)$ are assumed to be independent. The goal of the spectrum sensing is to decide between two hypotheses, $H_{0}$ and $H_{1}$, the hypothesis that the primary user is absent and present, respectively.

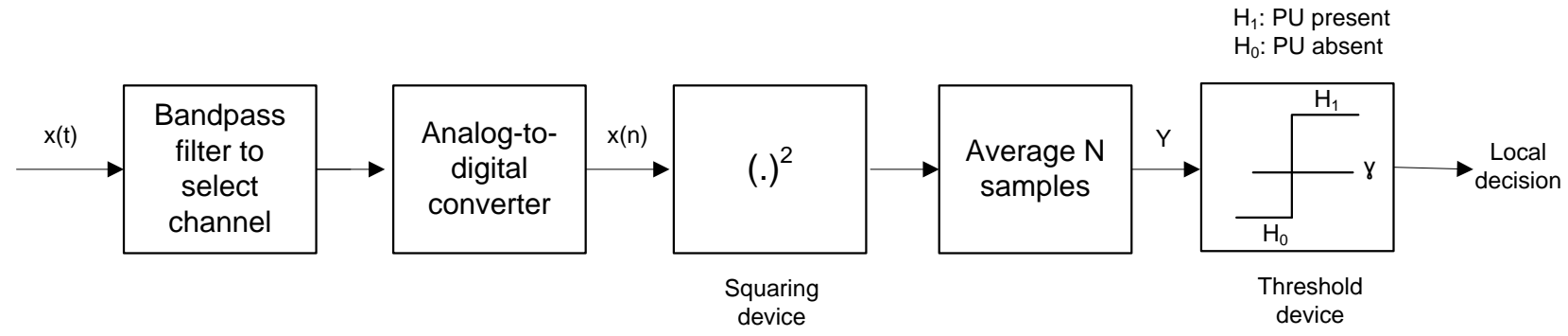

Figure 3.2: Block diagram of energy detector.

The test statistic for the energy detector for the $k^{\text {th }}$ user, $Y_{k}$, is computed as the sum of the received signal energy over an interval of $N$ samples, and is given by [49]:

$$
Y_{k}=\sum_{n=1}^{N}\left|x_{k}(n)\right|^{2} .
$$

Under hypothesis $H_{0}$, the test statistic $Y_{k}$ is a random variable whose probability density function is a Chi-square $\chi^{2}$ distribution with $N$ degrees of freedom; otherwise, $Y_{k}$ follows a non-central Chi-square $\chi^{2}$ distribution with $N$ degrees of freedom and parameter $\Gamma_{k}^{\prime}[38,51]$. The received SNR of the primary user measured at the $k^{t h}$ secondary user of interest, $\Gamma_{k}^{\prime}$, is given by

$$
\Gamma_{k}^{\prime}=\frac{\left|h_{k}\right|^{2} \sigma_{s}^{2}}{\sigma_{v}^{2}}
$$

For a large number of samples, $N(N \geq 10$ [37]), using the central limit theorem [82], 
the distribution of the test statistics, $Y_{k}$, can be approximated by a Gaussian distribution with mean [51]

$$
E\left(Y_{k}\right)= \begin{cases}N \sigma_{v}{ }^{2}, & H_{0} \\ N\left(\frac{\left|h_{k}\right|^{2} \sigma_{s}{ }^{2}}{\sigma_{v}{ }^{2}}+1\right) \sigma_{v}{ }^{2}, & H_{1}\end{cases}
$$

and variance

$$
\operatorname{Var}\left(Y_{k}\right)= \begin{cases}2 N \sigma_{v}^{4}, & H_{0} \\ 2 N\left(\frac{2\left|h_{k}\right|^{2} \sigma_{s}{ }^{2}}{\sigma_{v}{ }^{2}}+1\right) \sigma_{v}{ }^{4}, & H_{1}\end{cases}
$$

In this chapter, we consider secondary users with heterogeneous sensing abilities that are represented by different probabilities of detection and false alarms, $P_{d, k}$ and $P_{f, k}$, that are respectively given by

$$
P_{d, k}=P\left(Y_{k}>\gamma_{k} \mid H_{1}\right)=Q\left(\frac{\gamma_{k}-N\left(\sigma_{v}^{2}+\left|h_{k}\right|^{2} \sigma_{s}^{2}\right)}{\sqrt{2 N\left(\sigma_{v}^{2}+2\left|h_{k}\right|^{2} \sigma_{s}^{2}\right) \sigma_{v}^{2}}}\right)
$$

and

$$
P_{f, k}=P\left(Y_{k}>\gamma_{k} \mid H_{0}\right)=Q\left(\frac{\gamma_{k}-N \sigma_{v}^{2}}{\sqrt{2 N \sigma_{v}^{4}}}\right)
$$

where $\gamma_{k}$ is the energy detection decision threshold for the $k^{\text {th }}$ secondary user.

\subsubsection{Primary User Traffic Activity Model}

Information about primary users can benefit spectrum sensing and provide opportunity for other applications. Modelling of primary user traffic depends on how the PUs and SUs are

envisioned to operate. The statistical model of the primary user traffic should be simple enough to allow the design of optimal higher order protocols, but sufficiently accurate to describe the behaviour of the PU. The most common approach models PU activity with an alternating ON-OFF model $[15,58,79,83,84]$. In this model, each channel alternates between two states: ON state, in which the channel is occupied by a primary user and OFF state, in which the channel is idle as shown in Figure 3.3.

The exponential model is a common and generic model for the duration of the PU states 


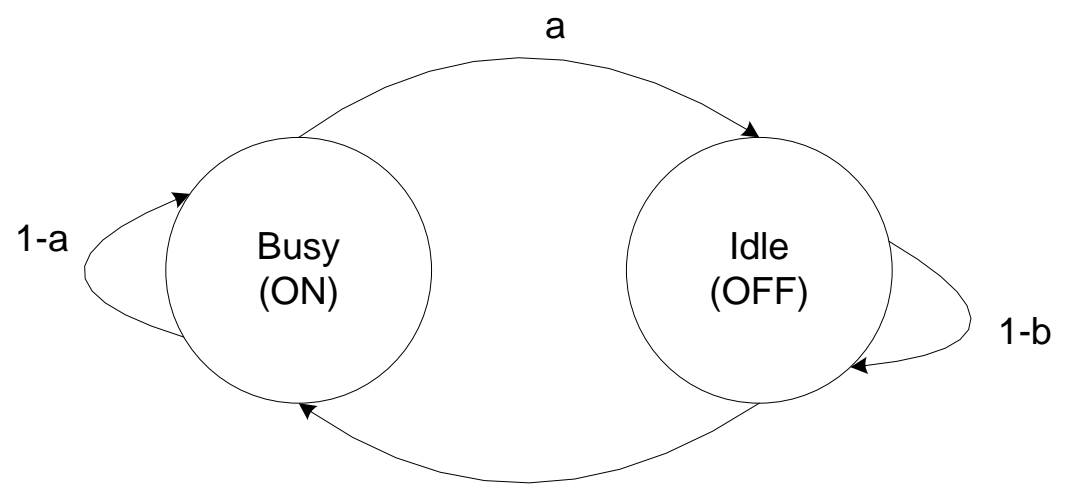

b

Figure 3.3: An ON-OFF model for primary users' channel usage.

and a good approximation of many practical scenarios $[85,86]$. Therefore, the durations of the $\mathrm{ON}$ and the OFF periods are assumed to be exponentially distributed and independent with means $1 / a$ and $1 / b$, respectively, and the probability density functions (pdf) for the $\mathrm{ON}$ and $\mathrm{OFF}$ states are respectively,

$$
f_{B}(t)=a e^{-a t}, \quad t \geq 0
$$

and

$$
f_{I}(t)=b e^{-b t}, \quad t \geq 0
$$

Therefore, the behavior of the PU is represented by two parameters, $a$, the rate of transition from $\mathrm{ON}$ state to $\mathrm{OFF}$ state and $b$, the rate of transition from OFF state to ON state. The steady state probability for the channel to be busy is [83],

$$
P_{B}=\frac{b}{a+b}
$$

and the steady state probability for the channel to be idle is,

$$
P_{I}=\frac{a}{a+b}
$$




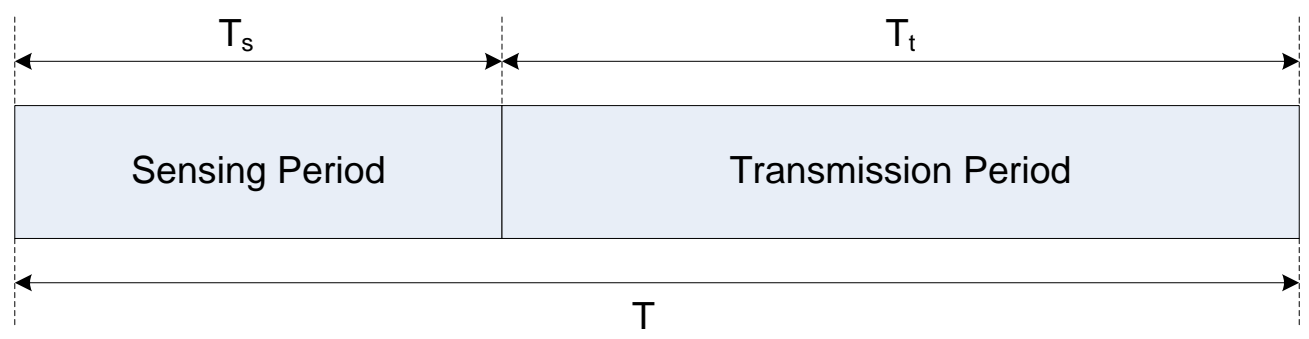

Figure 3.4: Frame structure for secondary users.

The secondary users can utilize the OFF period of the primary user channel to transmit their own data. We assume that each SU has only one transceiver, i.e., an SU cannot transmit data and perform sensing at the same time [15,21]. Therefore, each SU employs periodic time frames of length $T$ for sensing and transmission. The time frame structure for each secondary user is shown in Figure 3.4. Each frame consists of a sensing period $T_{s}$ required for observation, local decision making and reporting and a transmission period $T_{t}$ for data transmission in case the primary user is absent. During data transmission within a frame, if the transmission time, $T_{t}$, is relatively short, the spectrum state does not change during $T_{t}[58,61]$.

\subsection{Adaptive Group-based Cooperative Sensing Scheme}

According to the number of sensed channels in one sensing period, cooperative spectrum sensing could be broadly categorized into sequential and parallel cooperative sensing. In sequential cooperative sensing, all the cooperative SUs are scheduled to sense an identical channel in each sensing period and channels are sensed one by one sequentially. The sequential CSS scheme exploits the multiuser diversity leading to improved sensing accuracy on the expense of reduced sensing efficiency. An example of the sequential CSS is illustrated in Figure 3.5.

In parallel cooperative sensing, more than one channel is sensed in each sensing period. The cooperative SUs are divided into multiple groups while each group senses a different 

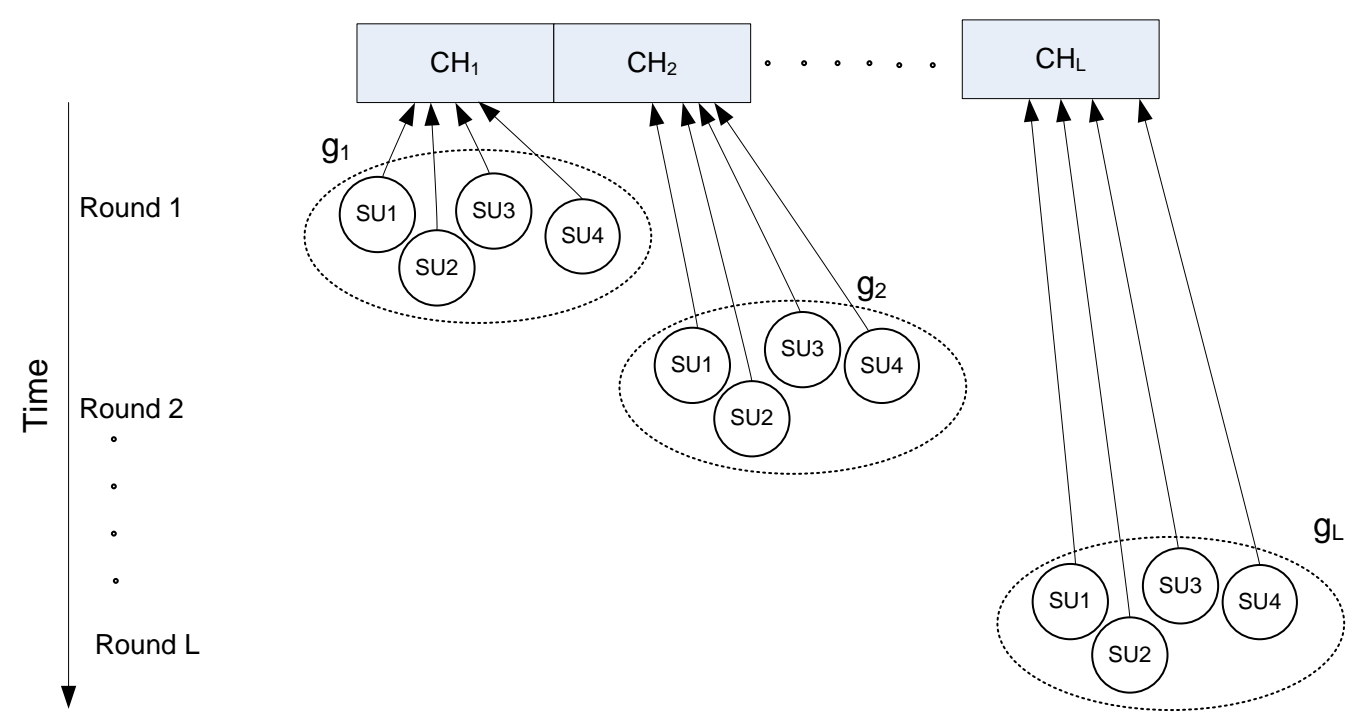

Figure 3.5: Illustration of sequential cooperative spectrum sensing scheme.

channel simultaneously. The motivation of parallel cooperative sensing is to enhance the sensing efficiency by allowing the cooperative SUs to sense distinct channels in one sensing period. Since multiple channels are detected in one sensing period, the time required to sense all the available channels is much shorter than that in sequential CSS and the sensing efficiency is significantly enhanced.

In this chapter, we consider a group-based cooperative spectrum sensing scheme for secondary users with heterogeneous sensing ability in terms of sensing accuracy. In this scheme, the secondary users in the same group cooperate together to sense the targeted channel using energy detection. Cooperative secondary users, with heterogeneous sensing abilities represented by non-identical probabilities of detection and false alarm, will be adaptively assigned to groups to achieve a trade-off between the sensing efficiency and the sensing accuracy. An example of the proposed adaptive group-based cooperative spectrum sensing scheme is illustrated in Figure 3.6.

Different from [62], we study the case in which the total number of groups is equal to the number of licensed channels, i.e., $|G|=L$, where $G=\left\{g_{1}, \cdots, g_{|G|}\right\}$ is the set of all groups and $|G|$ is the cardinality of set $G$. Each group, $g_{i}(i=1, \cdots,|G|)$, is used to sense a distinct 


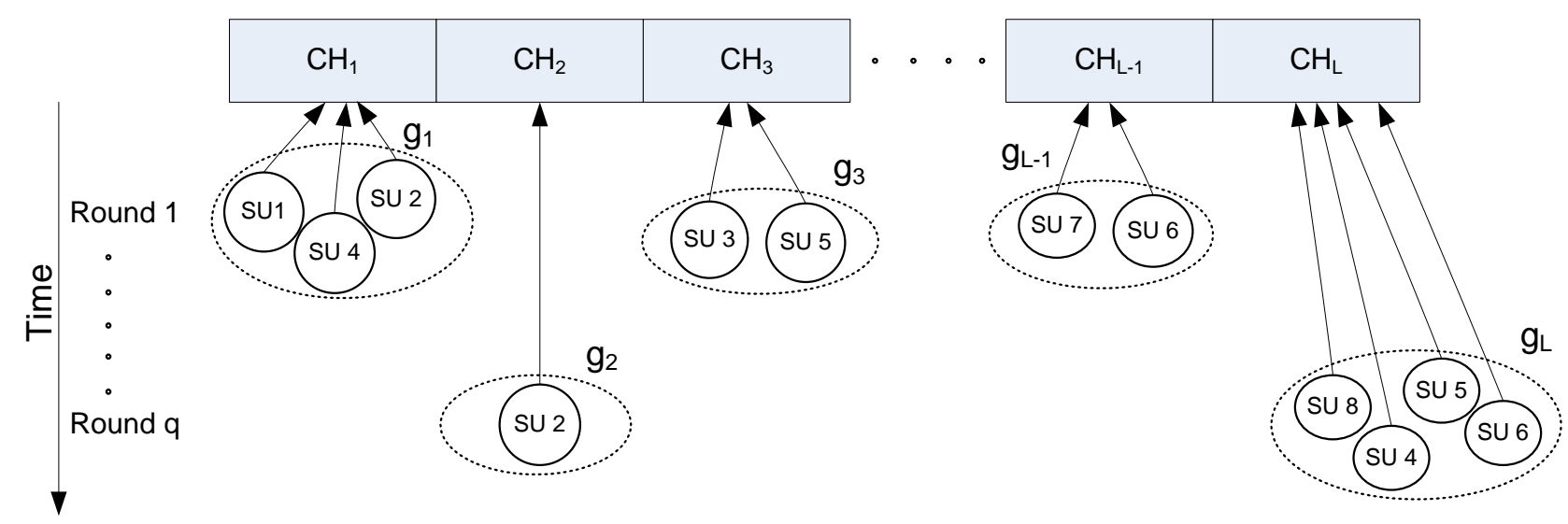

Figure 3.6: Illustration of the group-based cooperative sensing scheme.

channel. However, the number of groups used in each sensing round (the number of licensed channels that can be sensed in each sensing round) will differ based on how the cooperating secondary users are assigned to the different groups. After each sensing period, the sensing results are sent back to the fusion center. The fusion center will combine the results for each group to determine which channels are available. Without loss of generality, we assume that the fusion center uses the OR fusion rule to combine the decisions from different users for each group. In the OR fusion rule, the fusion center decides that the primary user is absent only if all the cooperating secondary users decide the absence of the primary user's signal as explained in section 2.5.2. If no idle channel is found in the first sensing round, more sensing rounds are performed until an idle channel is discovered or all the available $L$ channels are sensed. If an idle channel is discovered, the SU that reserved the channel can access the discovered channel and transmit its data. The average transmission time for a secondary user using an idle channel is equal to the average duration of the channel idle time, i.e., $T_{t}=\frac{1}{b}$.

In this work, we do not implicitly assume that SUs are willing to cooperate for spectrum sensing if they do not have packets to send, but rather we consider the more general case where secondary users can be assigned to perform cooperative sensing even when they have data to transmit $[61,73]$. We consider two scenarios depending on the availability of channel 
state information (CSI). In the first scenario, CSI is absent and we consider a fixed transmission rate $R$ on a channel used by a secondary user. In the second scenario, CSI is available and the secondary user can adapt the transmission rate according to the channel quality using adaptive modulation. In this work, we consider secondary users with low mobility; therefore, the channel gain of each transmission link can be estimated accurately via pilot symbols or training sequences [8]. Since a secondary user can be active only when there is no primary user activity, using the background noise and transmit power, the achievable transmission rate of a secondary user over each channel can be determined based on the physical layer model and parameters [87].

\subsubsection{Fixed Rate Transmission}

Suppose a secondary user, $k^{\prime}$, wants to find an idle channel to initiate data transmission. To find an idle channel, the secondary users will cooperatively sense the licensed channels using the group-based cooperative sensing scheme. If the secondary user, $k^{\prime}$, is unaware of the channel conditions of the discovered channel, it will transmit its data at a fixed rate. Let $R$ denote the rate over the channel if it is used by the secondary user, $k^{\prime}$. We define the average opportunistic throughput, $F$, of SU $k^{\prime}$ that gained access to the available channel during the transmission time, $T_{t}$, as

$$
F=\sum_{q=1}^{Q} P_{q}\left(g_{i}\right) R \frac{T_{t}}{q\left(T_{s}+T_{t}\right)}
$$

where $T_{s}$ is the duration of the sensing period in each frame, $q$ is the index of the sensing round and $Q$ represents the total number of sensing rounds needed to sense all the licensed channels where $1 \leq Q \leq|G|$. The term $P_{q}\left(g_{i}\right)$ is the probability that at least one group, $g_{i}$, was successful in discovering an available channel after $q$ sensing rounds. For a given $T_{s}$ and $T_{t}$, the average opportunistic throughput will decrease as the delay in finding a vacant channel increases which takes into account the effect of using multiple rounds on the 
throughput performance of the proposed scheme.

Since secondary users use a periodic sensing-transmission structure as mentioned earlier, they do not sense and transmit their data at the same time. Therefore, there will be a throughput cost incurred as a result of the cooperative spectrum sensing which we refer to in this thesis as sensing overhead. This sensing overhead will depend on the number of cooperating secondary users in each sensing round and the number of sensing rounds needed to discover a vacant channel. We define the average sensing overhead incurred by the group-based CSS scheme, $O$, as

$$
O=\sum_{q=1}^{Q} P_{q}\left(g_{i}\right) \sum_{h=1}^{q} \sum_{g_{i} \in G^{(h)}} \sum_{k \in g_{i}} R \frac{T_{s}}{T_{t}},
$$

where $G^{(h)}=\left\{g_{i}|1 \leq i \leq| G \mid\right\}$ is the set of groups that perform sensing in the $h^{\text {th }}$ sensing round.

Since the total number of groups, $|G|$, is equal to the number of licensed channels, $L$, and each group, $g_{i}$, is assigned to sense a distinct channel, $l$, in analysis that follows, sensing by group $g_{i}$ is equivalent to sensing channel $l$.

For the OR fusion rule, considering different secondary users have different probabilities of false alarm and detection, the probabilities of false alarm and detection of the final decision on the channel sensed by group $g_{i}$ are respectively given by,

$$
\begin{aligned}
& P_{F}\left(g_{i}\right)=1-\prod_{k \in g_{i}}\left(1-P_{f, k}^{i}\right), \\
& P_{D}\left(g_{i}\right)=1-\prod_{k \in g_{i}}\left(1-P_{d, k}^{i}\right),
\end{aligned}
$$

where $P_{f, k}^{i}$ and $P_{d, k}^{i}$ denote the probabilities of false alarm and detection of the $k^{\text {th }}$ user on the channel sensed by group $g_{i}$, respectively.

From (3.6) and (3.7), the target probability of false alarm for each channel, $P_{f, k}^{i}$, is related 
to the probability of detection, $P_{d, k}^{i}$, as follows:

$$
P_{f, k}^{i}=Q\left(\left(\sqrt{\frac{2\left|h_{k}^{i}\right|^{2} \sigma_{s}^{2}}{\sigma_{v}^{2}}+1}\right) Q^{-1}\left(P_{d, k}^{i}\right)+\sqrt{\frac{N}{2}} \frac{\left|h_{k}^{i}\right|^{2} \sigma_{s}^{2}}{\sigma_{v}^{2}}\right),
$$

where $h_{k}^{i}$ is the channel gain of the $k^{t h}$ user on the channel sensed by group $g_{i}$.

Let $P_{s}\left(g_{i}\right)$ represent the probability that a channel is successfully discovered by group $g_{i}$. Then, we have

$$
P_{s}\left(g_{i}\right)=\left(1-P_{F}\left(g_{i}\right)\right) P_{I} .
$$

Therefore, we calculate the probability that at least one channel is discovered by the adaptive group-based CSS scheme in the $q^{\text {th }}$ sensing round, $p_{q}$, as

$$
p_{q}=\sum_{x^{\prime}=1}^{\left|G^{(q)}\right|} \sum_{J_{\mid G(q)}^{\prime} \in} \prod_{\Upsilon^{\left(x^{\prime}\right)}}^{\left|G^{(q)}\right|}\left(P_{s}\left(u_{c, q}\right)\right)^{j_{c}}\left(1-P_{s}\left(u_{c, q}\right)\right)^{1-j_{c}}
$$

where $\left|G^{(q)}\right|$ is the cardinality of set $G^{(q)}, J_{\mid G^{(q) \mid}}^{\prime}=\left(j_{1}, \cdots, j_{\mid G^{(q) \mid}}\right)$ is a vector of length $\left|G^{(q)}\right|$ and $\Upsilon^{\left(x^{\prime}\right)}$ is a set of vectors where all elements are either 0 or 1 and the sum of the elements is equal to $x^{\prime}$, i.e., $Y^{\prime}=\left(y_{1}^{\prime}, \cdots, y_{m}^{\prime}\right) \in \Upsilon^{x^{\prime}}$ if $y_{\bar{r}}^{\prime} \in\{0,1\}, \forall 1 \leq \bar{r} \leq m$ and $\sum_{\bar{r}=1}^{m} y_{\bar{r}}^{\prime}=x^{\prime}$ [88]. The vector $U_{\mid G^{(q) \mid}}^{q}=\left(u_{1, q}, \cdots, u_{\left|G^{(q)}\right|, q}\right)$ is a vector of length $\left|G^{(q)}\right|$ and $u_{c, q} \in G^{(q)}$ such that $1 \leq c \leq\left|G^{(q)}\right|$ and $u_{c, q} \neq u_{d, q}, \forall q, c, d, c \neq d$. The probability that channel $l$ is successfully discovered by group $u_{c, q}, P_{s}\left(u_{c, q}\right)$, is given by $(3.17)$.

We calculate the probability that at least one channel is discovered after $q$ sensing rounds as

$$
P_{q}\left(g_{i}\right)=\frac{p_{1}}{1-\prod_{w=1}^{|G|}\left(1-p_{w}\right)}, \quad q=1
$$

and

$$
P_{q}\left(g_{i}\right)=\frac{p_{q} \prod_{u=1}^{q-1}\left(1-p_{u}\right)}{1-\prod_{w=1}^{|G|}\left(1-p_{w}\right)}, \quad q=2,3, \cdots,|G|,
$$

where $p_{q}$ is given by $(3.18)$ for $1 \leq q \leq|G|$. 


\subsubsection{Variable Rate Transmission}

To utilize the time-varying feature of the channels, we consider in this section that the secondary user can adapt its transmission rate according to the channel quality using adaptive modulation. According to Shannon theory, the channel rate and the received SNR have a one-to-one mapping relationship, i.e., $R_{i}=W \log _{2}\left(1+S N R_{i}\right)$, where $W$ refers to the channel bandwidth. Since adaptive modulation with finite states is more practical [89], we divide the SNR range into $M^{\prime}$ regions where region $m^{\prime}$ corresponds to an achievable rate $R_{m^{\prime}}$ and $m^{\prime}=1, \cdots, M^{\prime}$. Let $\mathbf{R}=\left\{R_{1}, R_{2}, \cdots, R_{M^{\prime}}\right\}$ represent the achievable channel rate vector of length $M^{\prime}$. Therefore, each secondary transmitter can select a rate according to its SNR on the channel [90].

For the variable transmission rate case, we express the average opportunistic throughput of the secondary user, $k^{\prime}$ that gained access to the channel during the transmission time, $T_{t}$, as

$$
F=\sum_{q=1}^{Q} P_{q}\left(g_{i}\right) R_{i, k^{\prime}} \frac{T_{t}}{q\left(T_{s}+T_{t}\right)},
$$

where the rate, $R_{i, k^{\prime}}$, is the channel rate achievable on the channel sensed by group $g_{i}$ for user $k^{\prime}$ when the sensed channel is free and is chosen for transmission and $R_{i, k^{\prime}} \in \mathbf{R}$.

We define the average sensing overhead incurred by the group-based CSS scheme for the case of variable rate transmission as

$$
O=\sum_{q=1}^{Q} P_{q}\left(g_{i}\right) \sum_{h=1}^{q} \sum_{g_{i} \in G^{(h)}} \sum_{k \in g_{i}} R_{i, k} \frac{T_{s}}{T_{t}}
$$

where $R_{i, k}$ is the rate with which the cooperating user $k$ was transmitting on its selected channel and $R_{i, k} \in \mathbf{R}$. If the cooperating user was not transmitting, we set $R_{i, k}=0$. 


\subsubsection{Problem Formulation}

Using the adaptive group-based CSS scheme, it is possible to sense more than one channel at each sensing period which can significantly increase the sensing efficiency of CSS. In this cooperative scheme, the non-identical cooperative secondary users are assigned to different groups and each group is responsible for sensing a distinct channel. Our objective is to optimally assign users to groups and then assign those groups to the sensing rounds such that the throughput efficiency is maximized. We define the throughput efficiency as the ratio of the average opportunistic throughput over the sum of the average opportunistic throughput and the average sensing overhead. To formulate this problem, we introduce the

user assignment indicator, $\xi_{i, k}$, and the group assignment indicator, $\eta_{i, q}$, where $i, k$ and $q$ are the indices of the groups, users and rounds, respectively. The user assignment indicator $\xi_{i, k}$ is equal to 1 if user $k$ is assigned to group $i$ and $\xi_{i, k}=0$, otherwise. Similarly, the group assignment indicator $\eta_{i, q}$ is equal to 1 if group $i$ is sensing in round $q$ and $\eta_{i, q}=0$, otherwise.

Therefore, we can express the average opportunistic throughput and the average sensing overhead incurred by the adaptive group-based CSS scheme as follows:

\section{For fixed rate transmission:}

$$
F=\sum_{q=1}^{|G|} P_{q}\left(g_{i}\right) R \frac{T_{t}}{q\left(T_{s}+T_{t}\right)}
$$

and

$$
O=\sum_{q=1}^{|G|} P_{q}\left(g_{i}\right) \sum_{h=1}^{q} \sum_{i=1}^{|G|} \sum_{k=1}^{K} \xi_{i, k} \eta_{i, h} R \frac{T_{s}}{T_{t}}
$$

For variable rate transmission:

$$
F=\sum_{q=1}^{|G|} P_{q}\left(g_{i}\right) \frac{T_{t}}{q\left(T_{s}+T_{t}\right)} \max _{i}\left(R_{i, k^{\prime}} \eta_{i, q}\right)
$$


and

$$
O=\sum_{q=1}^{|G|} P_{q}\left(g_{i}\right) \sum_{h=1}^{q} \sum_{i=1}^{|G|} \sum_{k=1}^{K} \xi_{i, k} \eta_{i, h} R_{i, k} \frac{T_{s}}{T_{t}}
$$

where $P_{q}\left(g_{i}\right)$ is given by (3.19) for $q=1$ and by (3.20) for $2 \leq q \leq|G|$.

We can express the probability that at least one channel is discovered in the $q^{\text {th }}$ sensing round, $p_{q}$, as

$$
p_{q}=\sum_{x^{\prime}=1}^{|G|} \sum_{J_{|G|}^{\prime} \in \Upsilon} \prod_{\left.x^{\prime} x^{\prime}\right)}^{|G|}\left(\eta_{i, q}\right)^{j_{i}}\left(P_{s}\left(g_{i}\right)\right)^{\eta_{i, q} j_{i}}\left(1-P_{s}\left(g_{i}\right)\right)^{\eta_{i, q}\left(1-j_{i}\right)}
$$

where $J_{|G|}^{\prime}$ and $\Upsilon^{\left(x^{\prime}\right)}$ are as defined in (3.18).

The probability of false alarm for each group is

$$
P_{F}\left(g_{i}\right)=1-\prod_{k=1}^{K}\left(1-P_{f, k}^{i}\right)^{\xi_{i, k}}
$$

Similarly, the probability of detection for each group is

$$
P_{D}\left(g_{i}\right)=1-\prod_{k=1}^{K}\left(1-P_{d, k}^{i}\right)^{\xi_{i, k}}
$$

We define the throughput efficiency, $\Gamma_{e f f}$, to be

$$
\Gamma_{e f f}=\frac{F}{F+O}
$$

We can now formulate the throughput efficiency maximization problem as follows:

$$
\max _{\xi_{i, k}, \eta_{i, q}} \quad \Gamma_{e f f}
$$

s. t. $\quad \xi_{i, k} \in\{0,1\} \quad 1 \leq i \leq|G|, 1 \leq k \leq K$

$$
\eta_{i, q} \in\{0,1\} \quad 1 \leq i \leq|G|, 1 \leq q \leq|G|
$$




$$
\begin{array}{cc}
\sum_{i=1}^{|G|} \xi_{i, k} \eta_{i, q} \leq 1, & 1 \leq q \leq|G|, 1 \leq k \leq K \\
\sum_{q=1}^{|G|} \eta_{i, q} \leq 1, & 1 \leq i \leq|G| \\
\left|\eta_{i, q}-\eta_{j, q}\right|=\min \left(1, \sum_{k=1}^{K} \xi_{i, k} \xi_{j, k}\right), & 1 \leq i, j, q \leq|G|, i \neq j \\
P_{D}\left(g_{i}\right) \geq P_{D t h}, & \forall g_{i} \in G
\end{array}
$$

Constraints (3.32) and (3.33) are to ensure proper values for the user and group assignment indicators, respectively. Since each user can sense at most one channel at a time, constraint (3.34) restricts the assignment of each user to only one group in each sensing round. However, to allow for more flexibility in the assignment, we do not restrict the assignment of the same user to a different group in another sensing round as shown in the example in Figure 3.6. Constraint (3.35) is to ensure that each group can sense in only one sensing round to ensure that each channel is sensed once. Constraint (3.36) indicates that the groups that do not share any users should be assigned to sense in the same sensing round. This constraint tends to maximize the number of groups in each sensing round in order to decrease the number of rounds needed to sense all the available channels which in turn reduces the delay in finding a vacant channel. Constraint (3.37) puts a limit on the probability of detection for each group $g_{i}, 1 \leq i \leq|G|$, to guarantee an adequate level of sensing accuracy.

\subsection{Adaptive Assignment Algorithm}

The optimization problem in (3.31)-(3.37) is a non-linear binary programming problem. This problem is computationally hard as it is more general and harder to solve than linear binary programming which is known to be $N P$-hard [91] (see Appendix A.2 for further details). In this section, we propose a heuristic adaptive algorithm to solve the formulated optimization 
problem. The pseudo code of the proposed algorithm is given in Algorithm 1.

In order to achieve our objective of maximizing the throughput efficiency with a guaranteed sensing accuracy on each channel, we need to maximize the opportunistic throughput while minimizing the sensing overhead. Both the opportunistic throughput and sensing overhead depend on the total number of sensing rounds needed to discover an idle channel and the number of cooperating users in each group. To this end, the proposed heuristic algorithm aims to minimize the number of sensing rounds needed to discover an available channels (by maximizing the number of groups (channels) sensed in each sensing round) and minimize the number of cooperating users in each group while satisfying a limit on the sensing accuracy for each group (channel). The proposed heuristic algorithm is constructed in three stages, which are (i) channel sorting,(ii) user assignment and (iii) group assignment for the variable transmission rate case. For the fixed transmission rate case, all the channels have the same rate $R$ so the channel sorting stage is not performed (Line 1 in Algorithm 1). Otherwise, the algorithm works the same for the user assignment and group assignment stages.

\subsubsection{Channel Sorting}

Since our aim is to maximize the throughput efficiency which can be achieved by maximizing the average opportunistic throughput of the secondary user while minimizing the overhead, finding an idle channel with a higher data rate using fewer sensing rounds is desirable. Accordingly, for the variable transmission rate case, we will select the channels to be sensed by arranging them in the descending order of their achievable rates. Therefore, for $i<j$, $R_{i, k^{\prime}} \geq R_{j, k^{\prime}}, \forall i, j$, where $R_{i, k^{\prime}}$ is the achievable transmission rate of the secondary user, $k^{\prime}$, searching for a vacant channel on the channel sensed by group $g_{i}$.

\subsubsection{User Assignment}

Since the cooperating secondary users have heterogeneous sensing abilities, we select the secondary users in each group according to their probability of detection on the channel 
sensed by this group. At the start of each round, the proposed algorithm compares the probability of detection, $P_{d, k}^{i}$, for each user, $k$, on all channels. The algorithm then assigns user $k$ to group $i$ for which it has the highest $P_{d, k}^{i}$. The assigned user is then removed from the set of all candidate users for this round. User $k$ can sense only one channel in each sensing round but can be assigned to sense another channel in a different round. By assigning users with the highest probability of detection to sense each channel, the sensing accuracy for each channel is increased with fewer number of users which will in turn decrease the average sensing overhead.

In the example shown in Figure 3.6, SU2 is assigned to sense channel 1 and channel 2, therefore, channel 1 and channel 2 must be sensed in different sensing rounds. On the other hand, channel 2 and channel $L$ are sensed by different secondary users and therefore can be sensed in the same sensing round.

\subsubsection{Group Assignment}

The proposed algorithm assigns users to a group (the groups are ordered according to the achievable rates of the channels) until the probability of detection on this channel reaches a certain target value, $P_{D t h}$; then, this group is removed from the set of all groups. This group will then be assigned to this sensing round and so the channel assigned to this group will be sensed in this sensing round (following that, the algorithm continues assigning users to the next group in the same fashion and so on). To maximize the number of groups in each sensing round, the algorithm will try to place any unassigned users in the remaining groups. For example, if after assigning users to groups 1 and 2 until $P_{D t h}$ is achieved, the remaining unassigned users can not meet this requirement for group 3. The proposed algorithm will then try to place those unassigned users in group 4 , then group 5 and so on until $P_{D t h}$ is achieved for a group, or it is determined that no more groups can be sensed in this sensing round. This is possible since each user has a different probability of detection for each group, $P_{d, k}^{i}$. Maximizing the number of groups in each round will in turn minimize the number of 
rounds needed to sense all available channels which will increase the average opportunistic throughput and accordingly increase the throughput efficiency.

\subsubsection{Complexity Analysis}

Since the main motivation for the proposed suboptimal algorithm is its reduced computational complexity, in this section, we quantify the time complexity of our heuristic algorithm (see Appendix A.3 for further details). First, we need to sort the channels according to their rates, as shown in line 1 , which requires time $(|G| \log (|G|))$ (this time is not required for the fixed transmission rate case). For each group, we need to sort the users according to their maximum probability of detection for this group which requires time $(K \log (K))$ in line 18 . Since we will have to return to line 17 until the condition on the probability of detection of the group in line 22 is satisfied, therefore, the complexity of the internal while-loop in line $17-28$ is $(K \log (K)+K)$.

In the first sensing round, $q=1$, the internal while-loop (line 17-28) is repeated for $|G|$ groups. In each subsequent sensing round, this internal while-loop will be repeated for all the remaining unassigned groups. In the worst case, the complexity will be the sum of the finite series: $[|G|(K \log (K)+K)+(|G|-1)(K \log (K)+K)+(|G|-2)(K \log (K)+K)+\ldots]$. This summation is equal to $\left(\frac{|G|}{2}\right)(|G|+1)(K \log (K)+K)$, therefore, the complexity of the adaptive assignment algorithm is approximately $O\left(|G|^{2} K \log (K)\right)$ for both cases of fixed and variable transmission rates. On the other hand, the exhaustive search has an exponential time complexity of $O\left(2^{\left(K|G|+|G|^{2}\right)}\right)$ which is very high (see Table A.1 for comparison of some common classes of time complexities). 


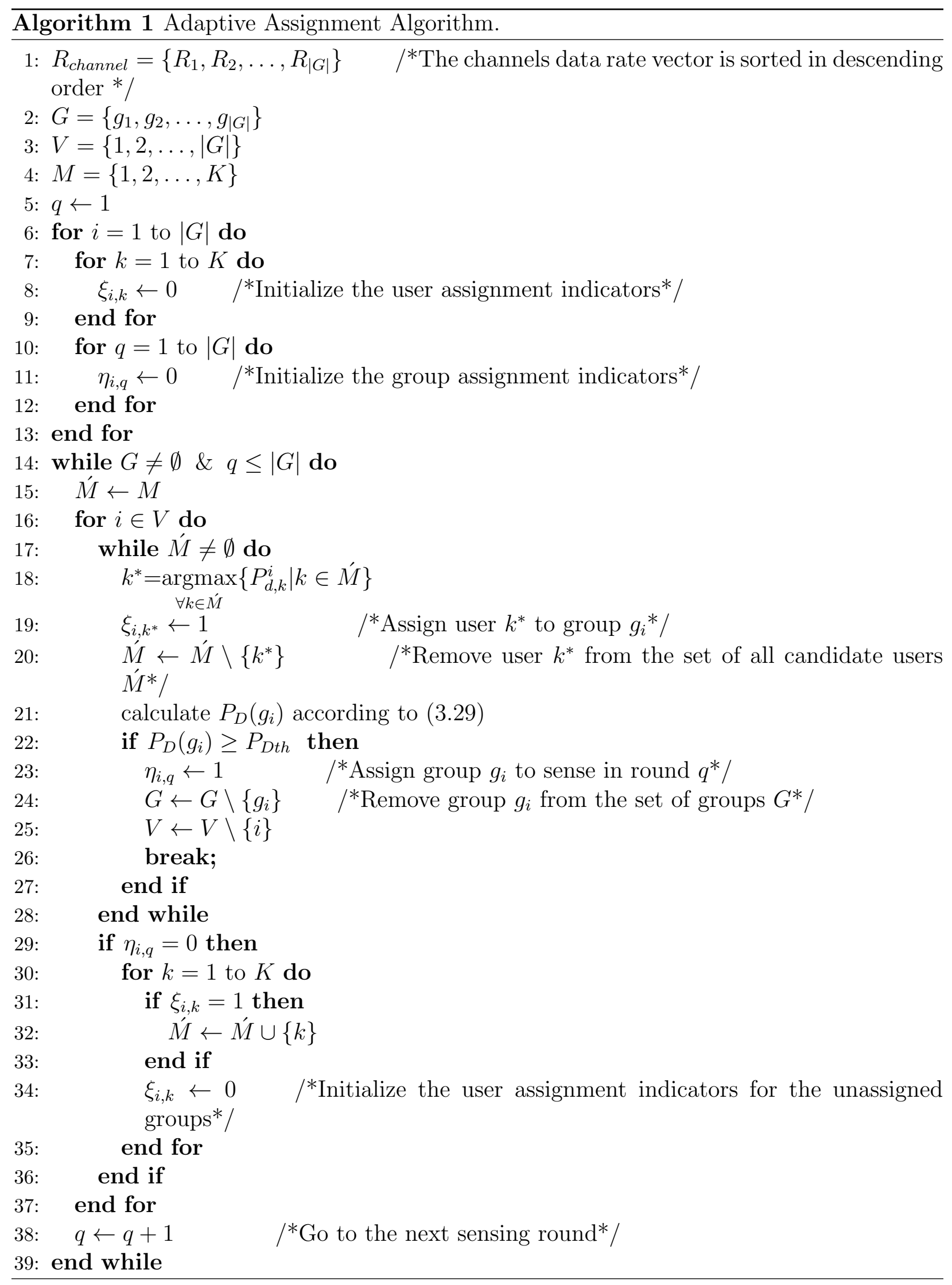




\subsection{Performance Evaluation}

In this section, we evaluate the performance of the proposed adaptive user and group assignment algorithm for both cases of fixed and variable transmission rates using Matlab. We consider a sensing period, $T_{s}$, of length $1 \mathrm{~ms}$ and the length of the sensing frame, $T$, is 10 ms [92]. Each channel has a bandwidth of $6 \mathrm{MHz}$ [93]. The threshold on the probability of detection, $P_{D t h}$, is set to 0.9 and the probability that the primary user is absent $P_{I}$ is set to 0.3 unless otherwise stated. The probability of detection for each user and channel, $P_{d, k}^{i}$, is randomly generated from a uniform distribution between $[0.5,1]$ and the number of samples $N=100$. For each $P_{d, k}^{i}$, the probability of false alarm, $P_{f, k}^{i}$, is calculated according to (3.16). The results are obtained by averaging over 1000 simulation runs.

We compare the proposed adaptive assignment algorithm with the non-adaptive grouping scheme and the sequential cooperative spectrum sensing scheme. In the non-adaptive grouping scheme, the secondary users are randomly assigned to groups to sense the channels, not based on their probability of detection on each channel. In the sequential cooperative sensing scheme, all SUs sense the same channel in each sensing period with the aim of improving the sensing accuracy of the primary user activity. Therefore, in sequential CSS, we

assign all the users to each group, i,e., $\sum_{k=1}^{K} \xi_{i, k}=K, \forall i$ and assign only one group to each sensing round.

\subsubsection{Fixed Rate Transmission}

For the fixed rate transmission, we evaluate the performance of the proposed algorithm with the parameters mentioned above when the transmission rate $R$ is equal to $10 \mathrm{Mb} / \mathrm{s}$. In Figure 3.7, we compare the throughput efficiency of the proposed algorithm, non-adaptive sensing scheme and sequential sensing scheme with the optimal throughput efficiency obtained through an exhaustive search when $|G|=2$ and $3 \leq K \leq 6$. The main drawback of exhaustive search is that its complexity increases exponentially with the number of secondary 
users, $K$ and number of groups, $|G|$. Due to this high time complexity, an exhaustive search could only be used in a domain where $K$ and $|G|$ are small. We observe from the figure that the throughput efficiency of the proposed algorithm is close to the maximum throughput efficiency obtained through exhaustive search with a relative difference bounded by $4.2 \%$. The relative difference between the exhaustive search and the non-adaptive scheme is much larger and can reach up to $14.2 \%$. It is also clear from the figure that the proposed adaptive algorithm outperforms both the non-adaptive and sequential algorithms for all the considered values of $K$. The degraded performance of the sequential CSS scheme with respect to the other two schemes is due to the large sensing overhead incurred by the sequential scheme.

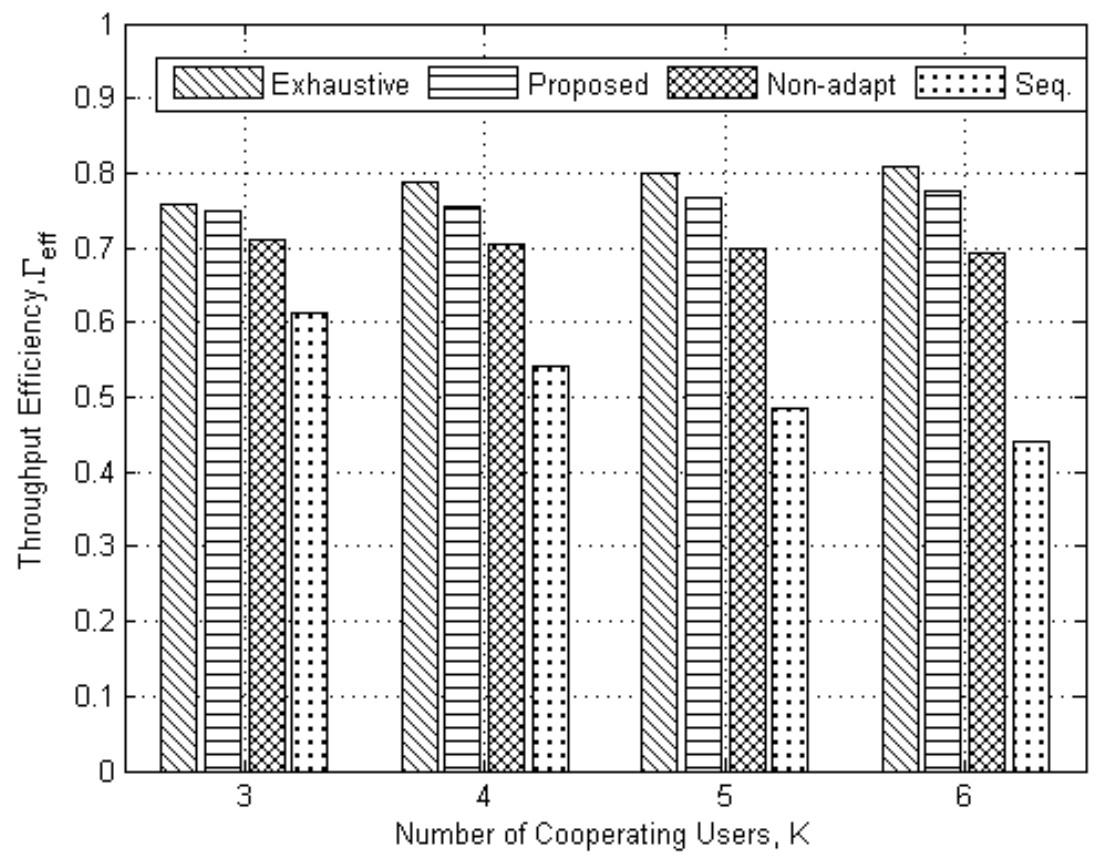

Figure 3.7: Comparision of throughput efficiency with different number of cooperating users for $|G|=2$.

In Figure 3.8, we plot the throughput efficiency versus the number of cooperating users $K$ with $|G|=4$ for the proposed adaptive assignment algorithm, non-adaptive and sequential CSS schemes. We observe that for the proposed algorithm, the throughput efficiency in- 


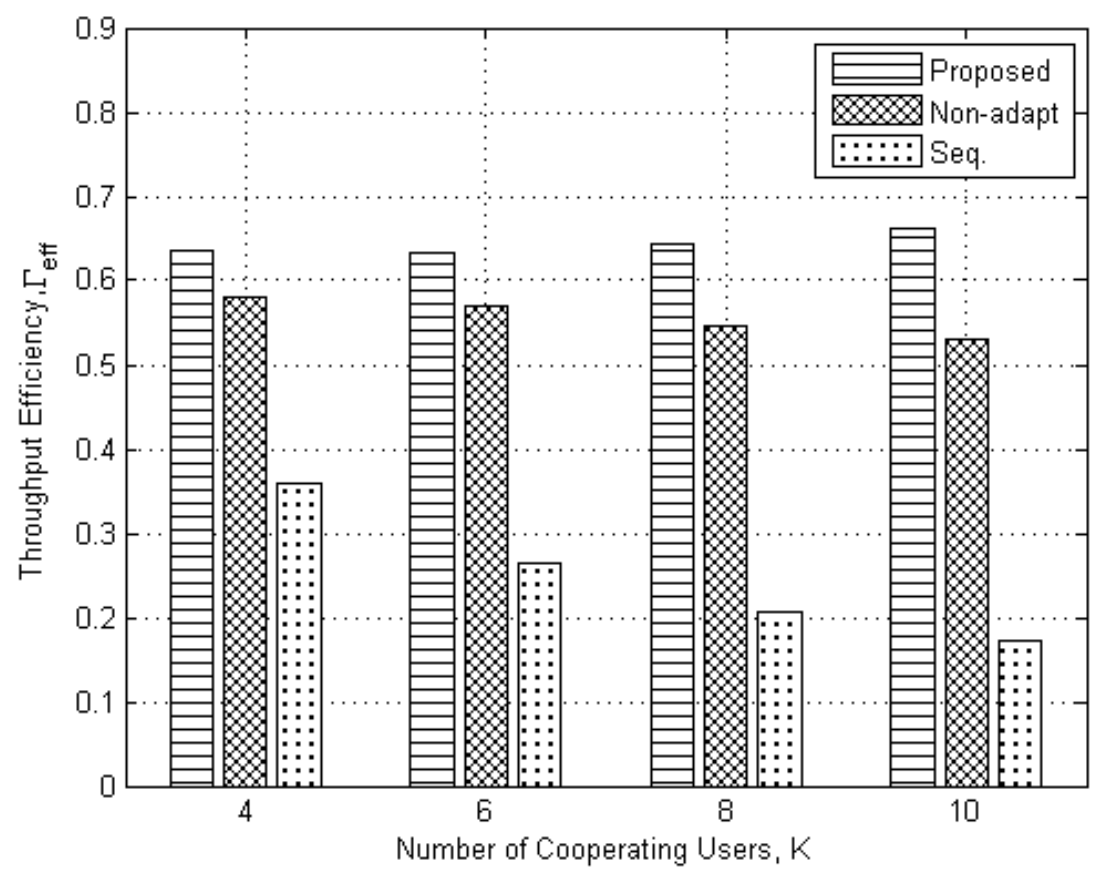

Figure 3.8: Throughput efficiency versus $K$ for $|G|=4$.

creases when the number of users increases with respect to the number of groups (channels) as this provides more degrees of freedom in the users assignment, which results in better performance of the adaptive scheme compared to the non-adaptive and sequential schemes. The relative difference between the proposed adaptive algorithm and the non-adaptive CSS scheme is approximately $20 \%$ for $K=10$ compared to a relative difference of approximately $9 \%$ for $K=4$. We also observe that the throughput efficiency for the sequential CSS scheme decreases as the number of users increases since the sequential scheme uses all the users to sense the same single channel which largely increases the sensing overhead and degrades the throughput efficiency. For $K=10$, the relative difference between the sequential CSS scheme and the proposed adaptive algorithm is approximately $74 \%$.

Figure 3.9 shows the comparison of throughput efficiency for the three sensing schemes with different number of groups with $K=2|G|$ in each case. The comparison indicates that the proposed algorithm is able to achieve higher throughput efficiency compared to the nonadaptive and sequential sensing schemes. As the number of available groups increases, while 


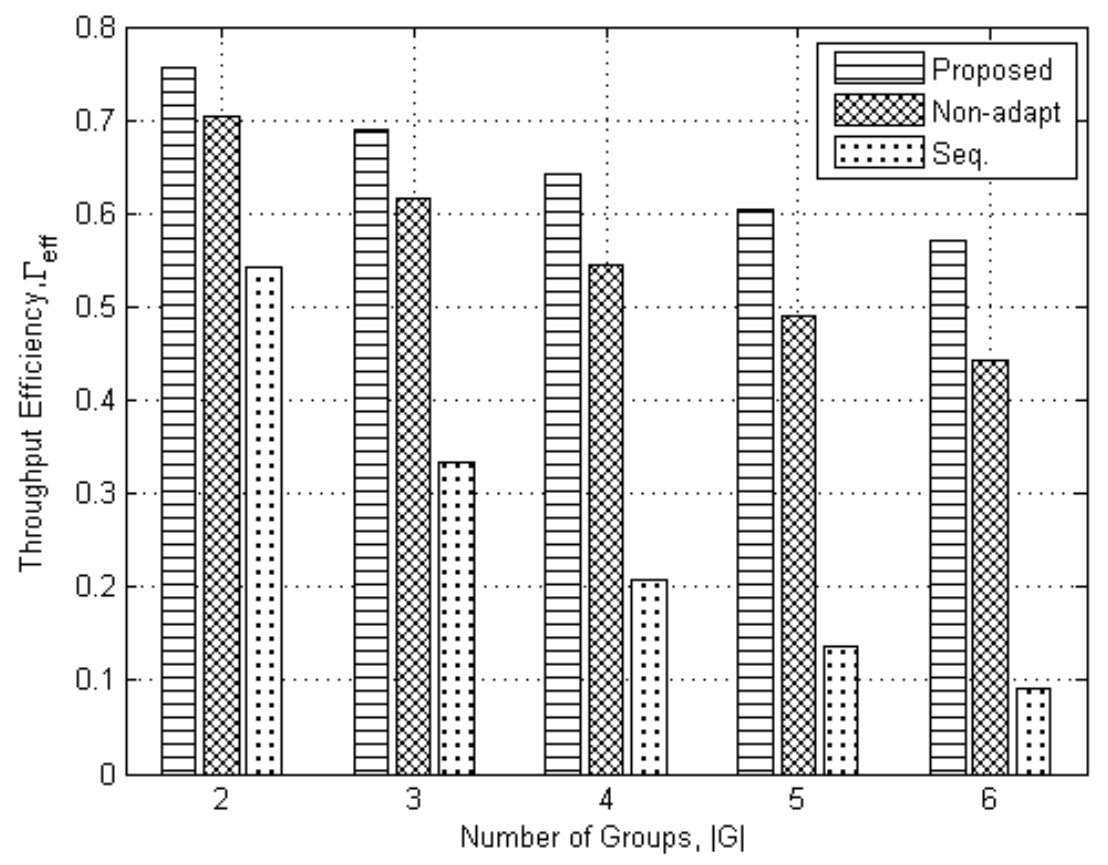

Figure 3.9: Throughput efficiency versus $|G|$ for $K=2|G|$.

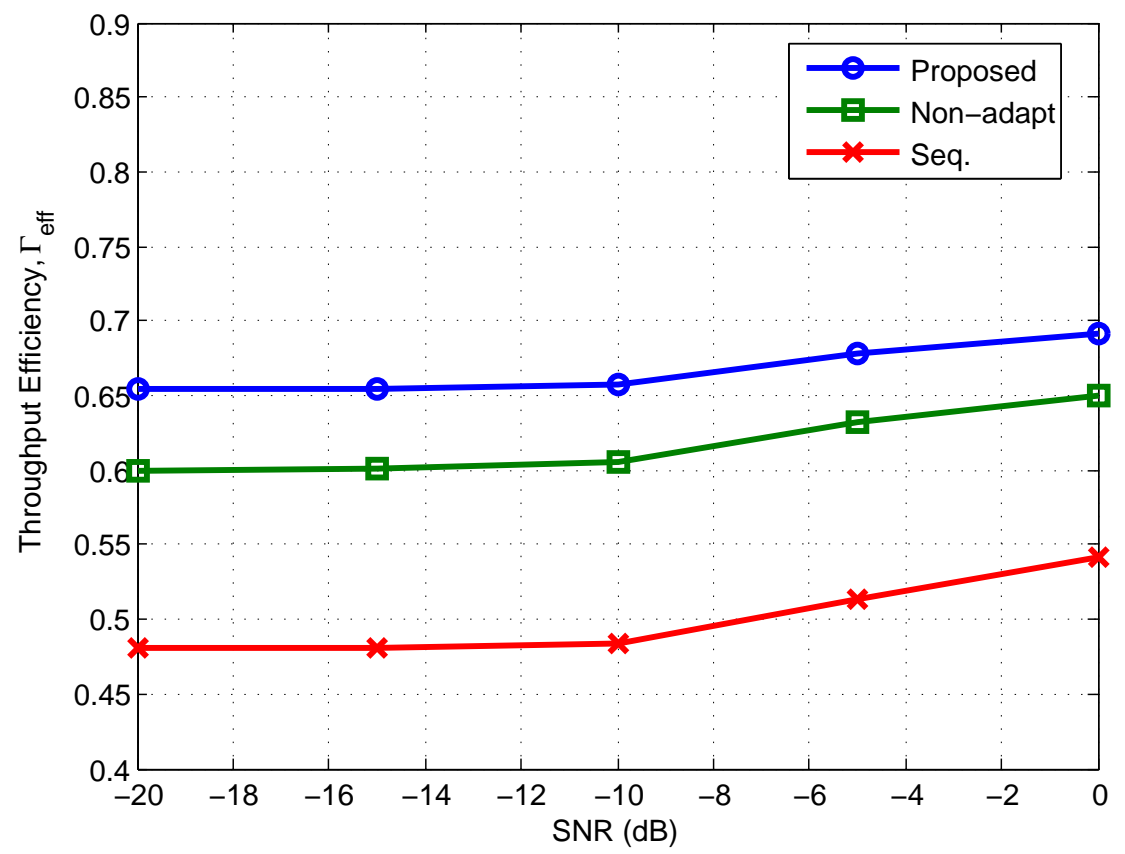

Figure 3.10: Throughput efficiency versus SNR for $|G|=K=3$. 
fixing the ratio between the cooperating users and the number of groups, the throughput efficiency for all three schemes decreases. This is because the average number of sensing rounds increases and the average probability of finding an available channel decreases, which in turn decreases the opportunistic throughput and increases the sensing overhead.

Figure 3.10 shows the throughput efficiency versus the SNR with $K=3$ and $|G|=3$ for the three schemes considered. We observe that the proposed algorithm is still able to provide the highest throughput efficiency compared to the non-adaptive and sequential schemes for different SNR. We also observe that, as the SNR increases the throughput efficiency increases for all three schemes. This is expected since for a fixed number of users and groups, increasing the SNR decreases the probability of false alarm for each group which in turn increases the average opportunistic throughput.

\subsubsection{Variable Rate Transmission}

For the variable transmission rate case, we evaluate the performance of the proposed algorithm for the number of transmission modes $M^{\prime}=5$ and the rate vector $\mathbf{R}=\{4.54,6.05,7.56$, 9.08, 12.10\} Mb/s [7]. In Figure 3.11, we compare the throughput efficiency of the proposed adaptive assignment algorithm, non-adaptive grouping and sequential sensing schemes with the optimal solution obtained by the exhaustive search when $|G|=2$ and $3 \leq K \leq 6$. We observe from the figure that the throughput efficiency of the proposed algorithm is close to the maximum throughput efficiency obtained through exhaustive search with a relative difference bounded by $2.75 \%$. On the other hand, the relative difference between the exhaustive search and the non-adaptive scheme is bounded by 11.5\%. We also observe from the figure that the proposed adaptive algorithm outperforms both the non-adaptive and sequential algorithms for all the considered values of $K$. In Figure 3.12, we plot the throughput efficiency versus the number of cooperating users $K$ with $|G|=4$ for the proposed adaptive assignment algorithm, non-adaptive grouping and sequential sensing schemes. We observe that for the proposed algorithm, the throughput efficiency increases when the number of users increases 


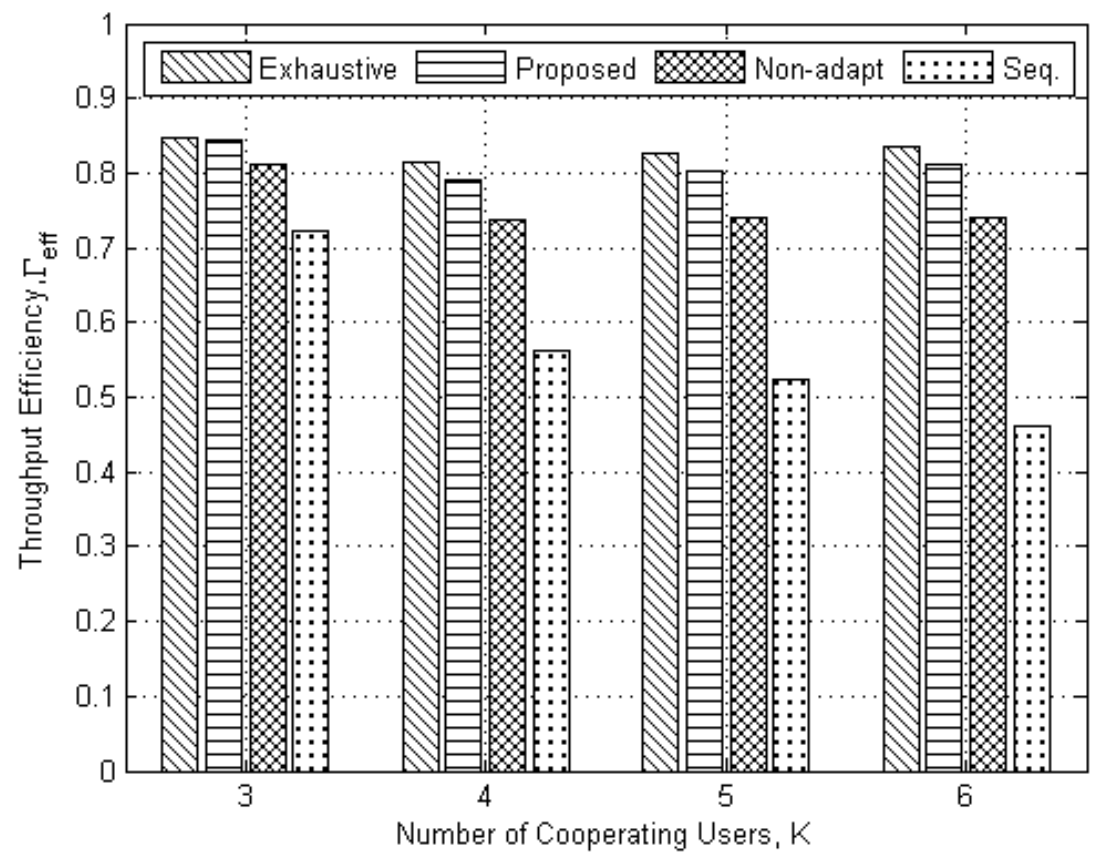

Figure 3.11: Throughput efficiency versus number of cooperating users for $|G|=2$.

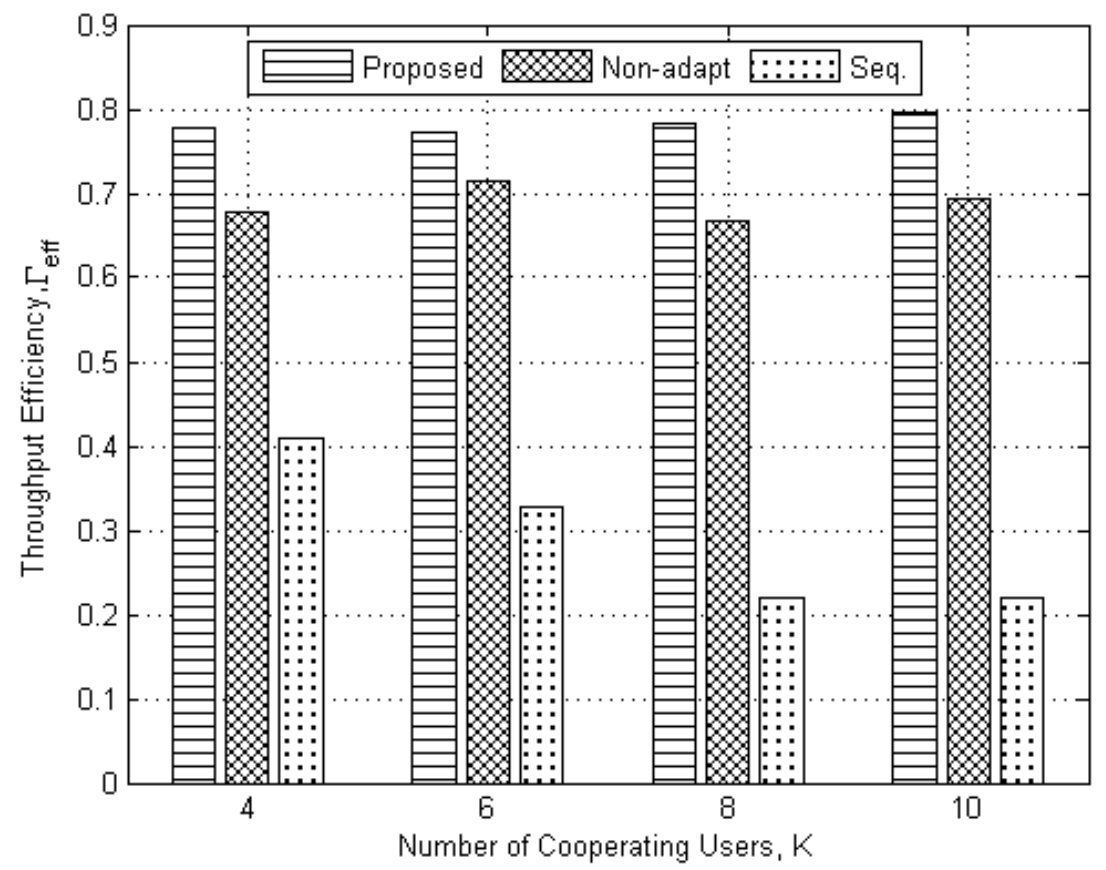

Figure 3.12: Throughput efficiency versus number of cooperating users for $|G|=4$. 
with respect to the number of groups (channels) as this provides more degrees of freedom in the users assignment, which results in better performance of the adaptive scheme compared to the non-adaptive and sequential schemes. The relative difference between the adaptive assignment algorithm and the non-adaptive algorithm is approximately $15 \%$ when $K=10$. For a fixed number of groups, the performance of the sequential CSS degrades significantly compared to the performance of the adaptive algorithm as the number of users increases resulting in a relative difference of approximately $47 \%$ and $72 \%$ when $K=4$ and $K=10$, respectively. Figures 3.13 and 3.14 show the average opportunistic throughput and average

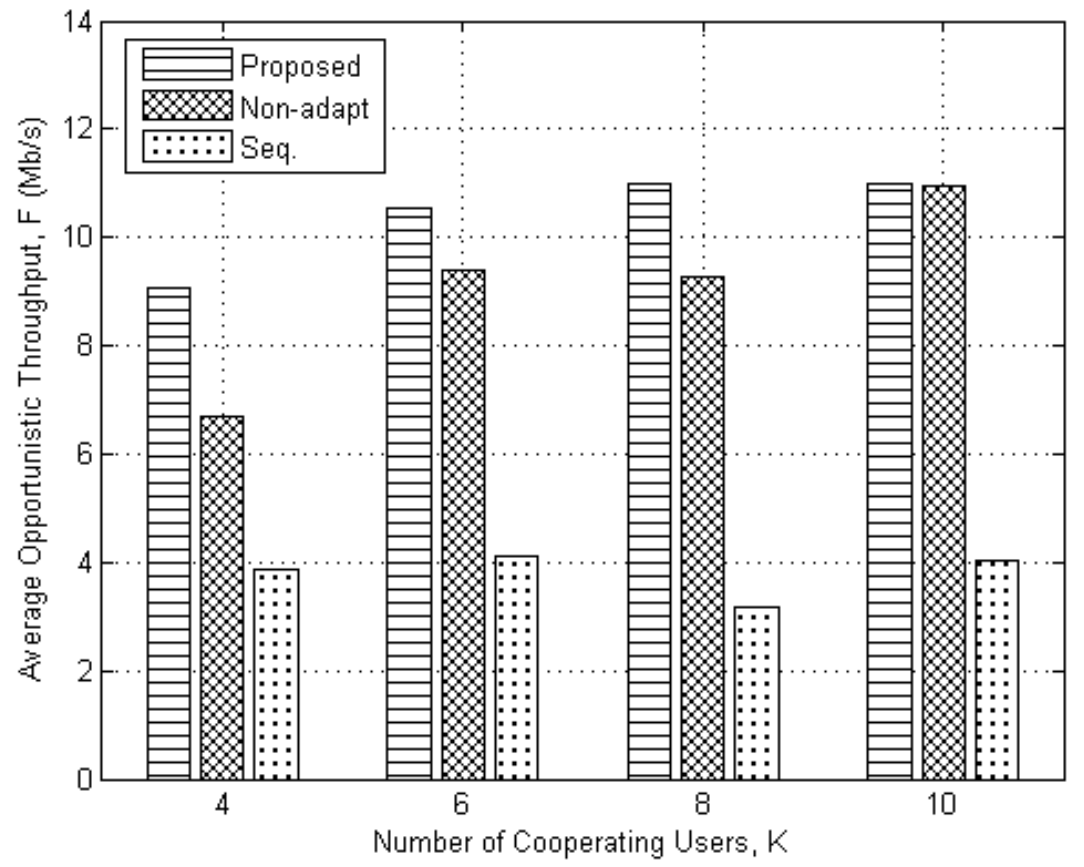

Figure 3.13: Average opportunistic throughput versus number of cooperating users for $|G|=$ 4 .

sensing overhead versus the number of cooperating users with $|G|=4$, respectively, for all three schemes. We observe from the figures that for the proposed algorithm, when the number of users compared to the number of channels is small $(K<8)$, both the average opportunistic throughput and the average sensing overhead increase with the number of users. However, when $(K \geq 8)$, there is slight increase in the average opportunistic throughput 


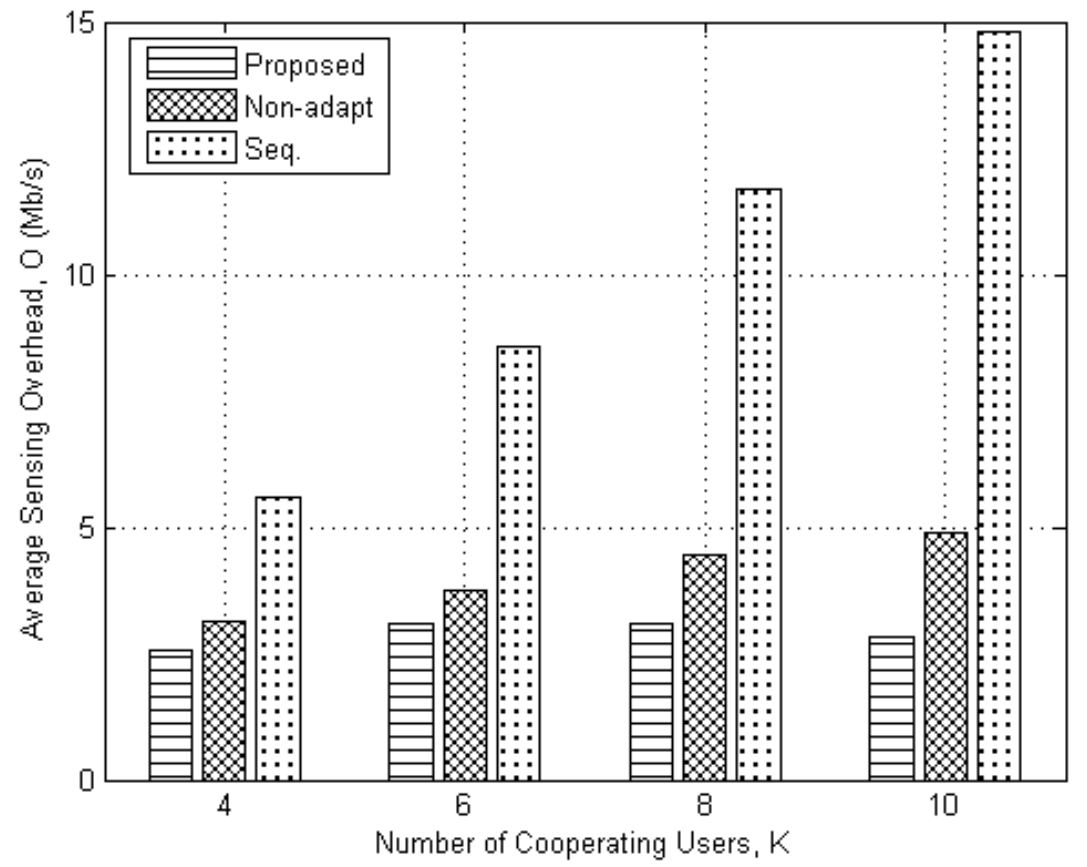

Figure 3.14: Average sensing overhead versus number of cooperating users for $|G|=4$.

while the average sensing overhead starts to decrease as more users are available, which allows for the best users (with higher probability of detection for the channels) to be chosen for cooperation and therefore, on average, less number of rounds are needed to discover the available channel. However, for the non-adaptive scheme, increasing the number of users increases the average sensing overhead as the users are randomly assigned; therefore, on average, more sensing rounds may be needed to discover the available channel as compared to the proposed scheme. The probability mass function of the number of sensing rounds for the proposed algorithm and the non-adaptive algorithm for $K=4$ and $K=8$ are shown in Figures 3.15 and 3.16, respectively. For the sequential scheme, the number of sensing rounds is fixed and is equal to the number of available channels. Figure 3.17 shows the comparison of throughput efficiency for all three schemes with different number of groups when fixing the number of users to twice the number of available groups. The comparison indicates that the proposed algorithm is consistently able to achieve higher throughput efficiency compared to the non-adaptive and sequential sensing schemes. We also notice that when the number of 


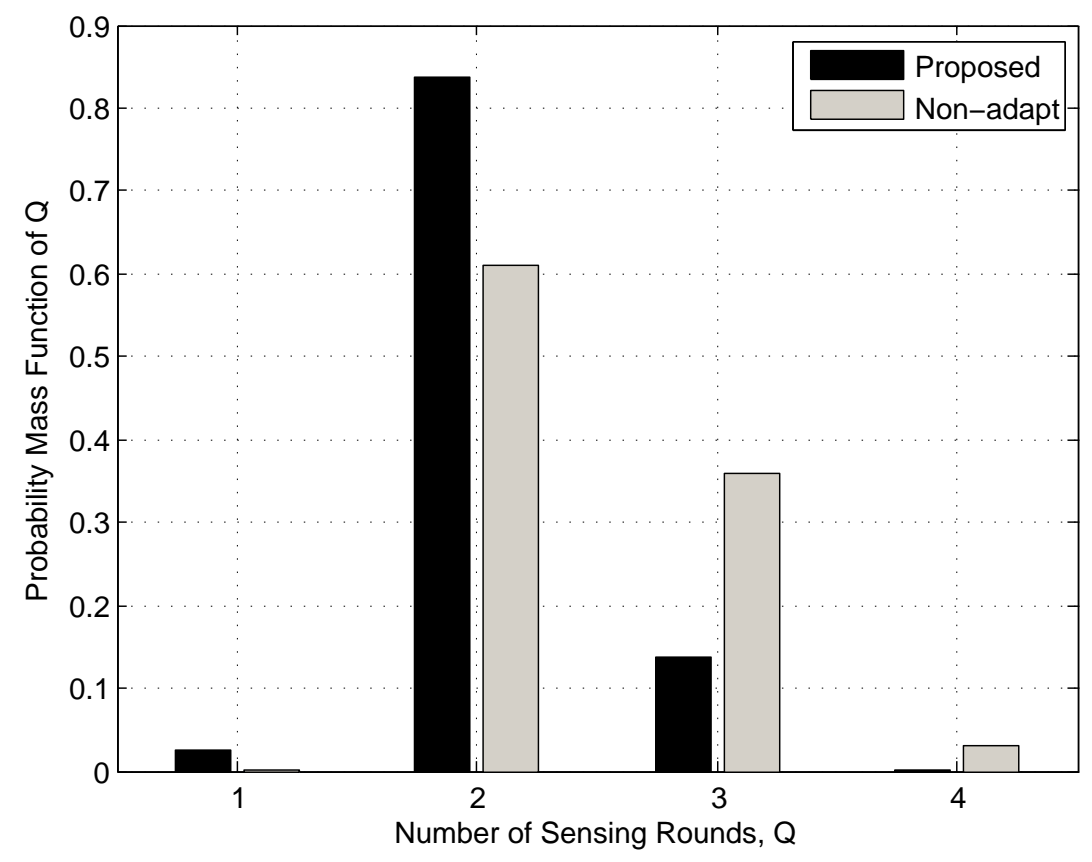

Figure 3.15: Probability mass function of the number of sensing rounds for the proposed and non-adaptive schemes for $|G|=4$ and $K=4$.

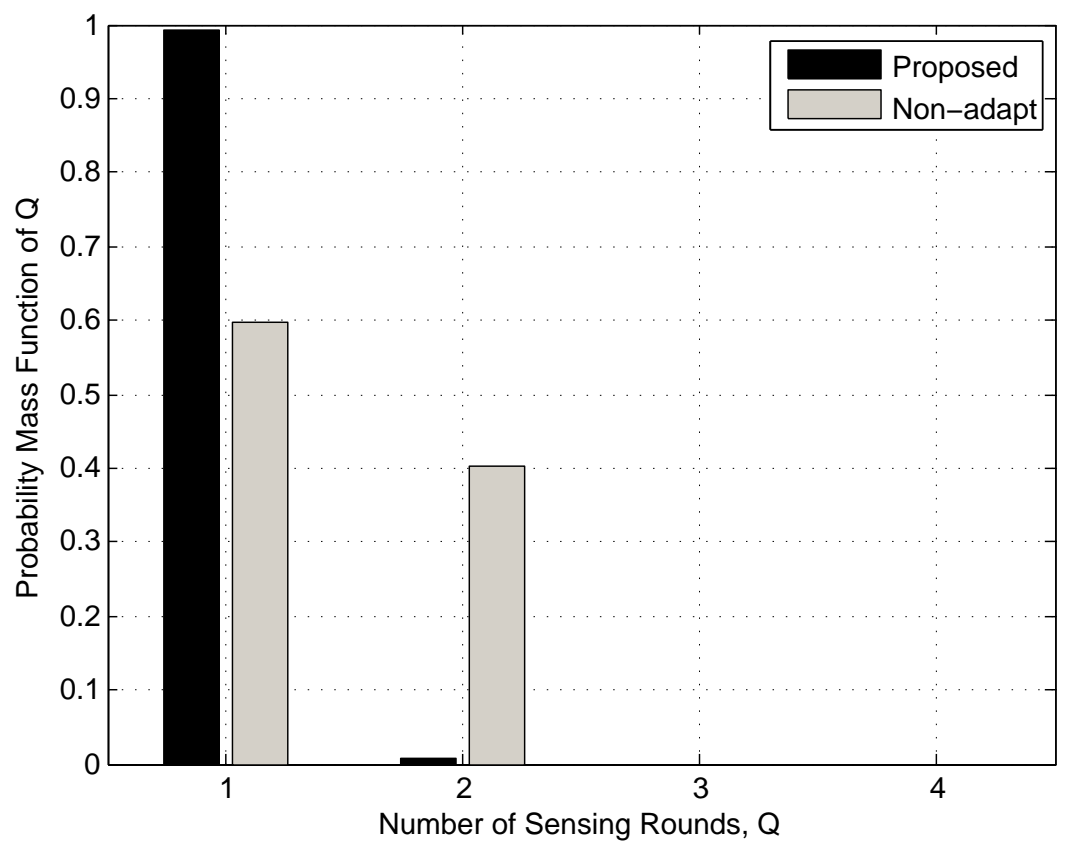

Figure 3.16: Probability mass function of the number of sensing rounds for the proposed and non-adaptive schemes for $|G|=4$ and $K=8$. 
groups and users increase, the relative difference between the proposed adaptive algorithm and the non-adaptive scheme also increases, because we have more degrees of freedom in assigning the users to the groups which is better exploited by the proposed adaptive assignment algorithm. For $|G|=6$ and $K=12$, the relative difference between the two schemes is approximately $19 \%$ compared to a relative difference of approximately $6.5 \%$ when $|G|=2$ and $K=4$. For $|G|=6$ and $K=12$, the relative difference between the proposed adaptive assignment algorithm and the sequential CSS scheme is approximately $86 \%$.

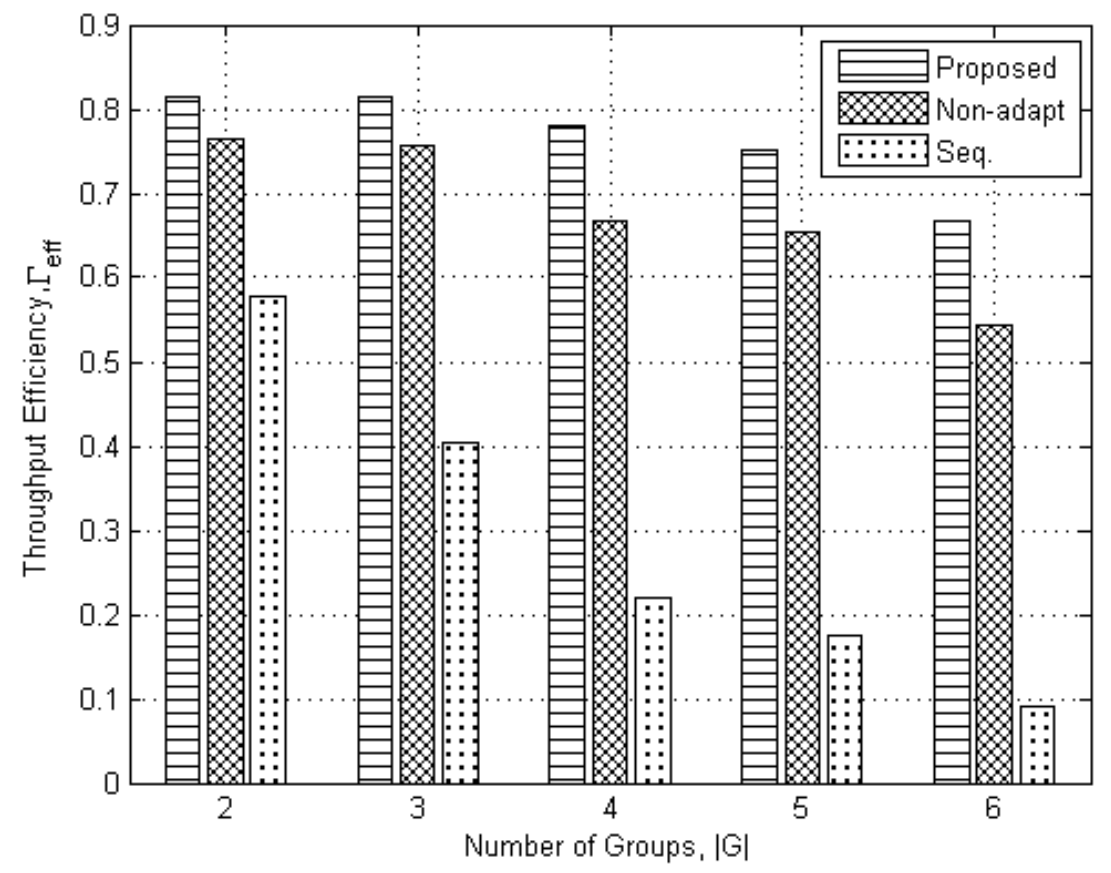

Figure 3.17: Throughput efficiency versus number of groups for $K=2|G|$.

In Figures 3.18 and 3.19, we compare the probability mass function of the number of sensing rounds for the proposed adaptive assignment algorithm and non-adaptive scheme for $|G|=3$ and $|G|=5$, respectively, and $K=2|G|$. Since the number of users are sufficiently larger than the number of groups, both algorithms were able to sense all the available channels in only two sensing rounds. However, the proposed adaptive algorithm can take better advantage of the heterogeneous sensing ability of the cooperating secondary users as compared to the non-adaptive CSS scheme. Therefore, the proposed algorithm is able to 


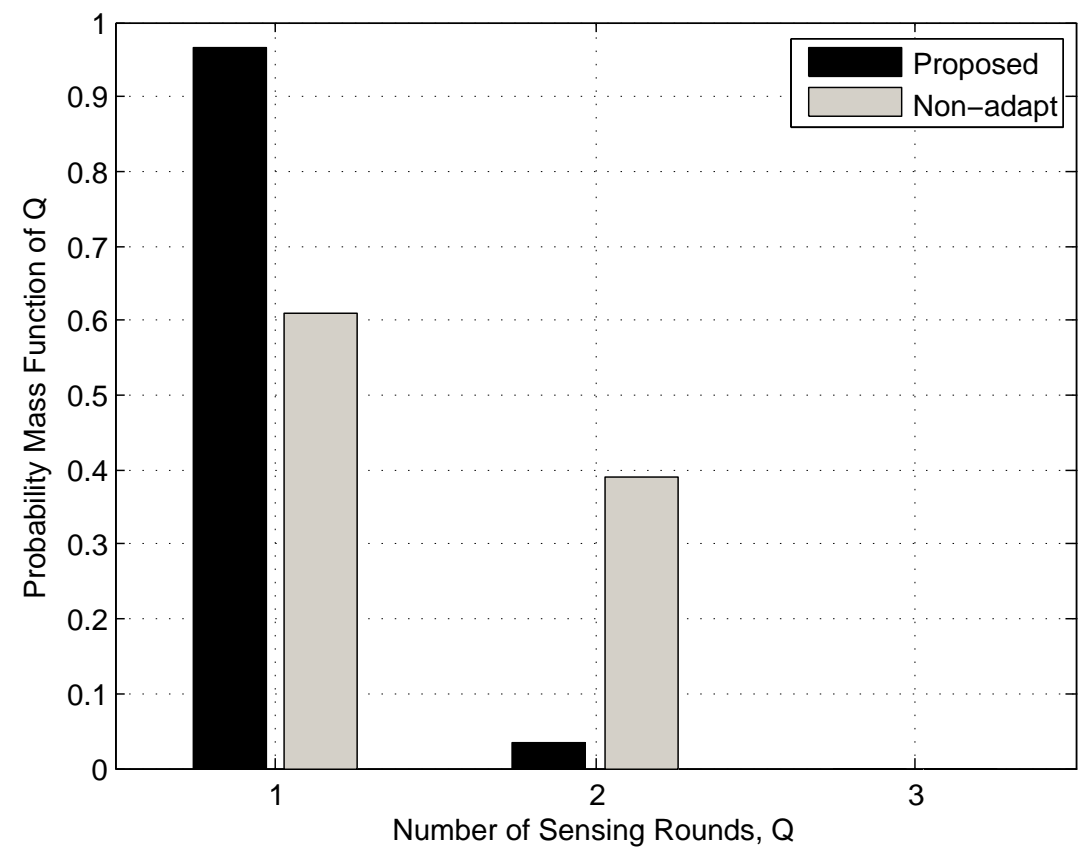

Figure 3.18: Probability mass function of the number of sensing rounds for the proposed and non-adaptive schemes for $|G|=3$ and $K=6$.

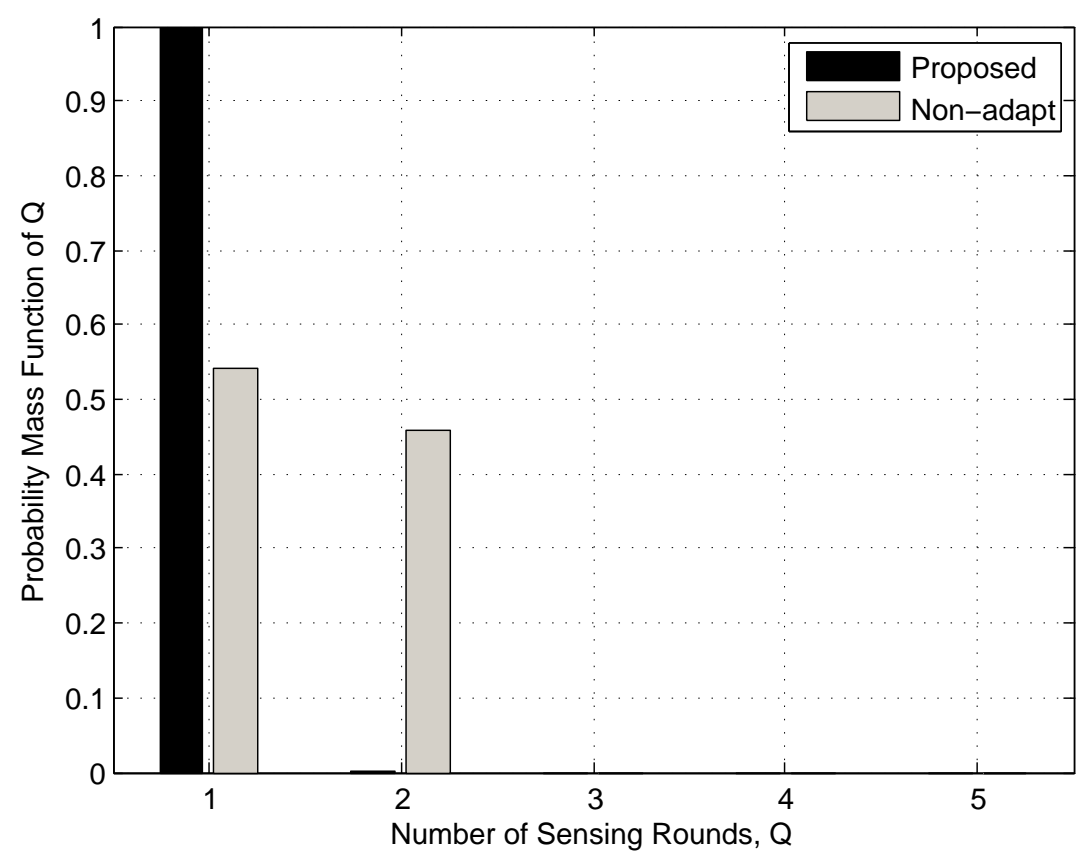

Figure 3.19: Probability mass function of the number of sensing rounds for the proposed and non-adaptive schemes for $|G|=5$ and $K=10$. 


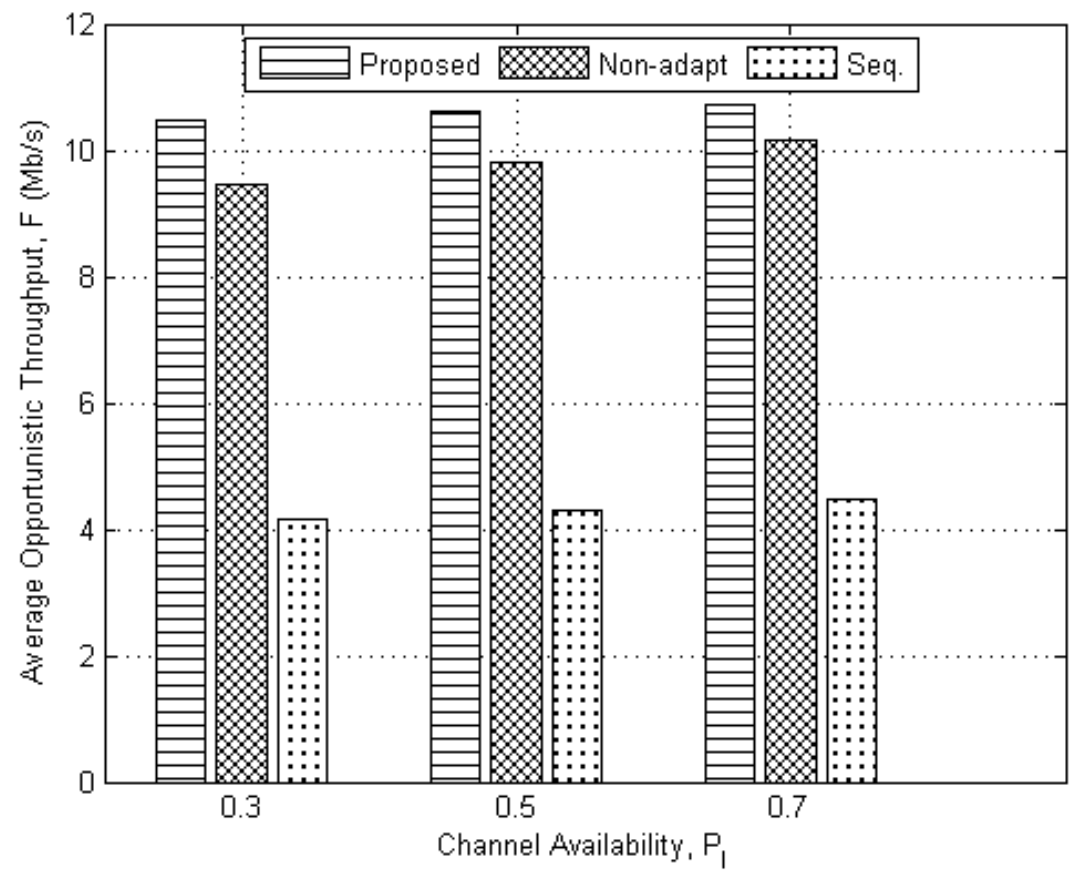

Figure 3.20: Average opportunistic throughput versus channel availability for $|G|=4$ and $K=6$.

use less number of sensing rounds to detect an available channel as compared to the nonadaptive scheme, especially when the number of available groups increases for almost half of the cases as shown in Figure 3.19. In Figures 3.20 and 3.21, we compare the average opportunistic throughput and average sensing overhead of the proposed, non-adaptive and sequential algorithm for different values of the channel availability $P_{I}$ for $|G|=4$ and $K=6$, respectively. The figures show that the proposed algorithm outperforms the non-adaptive and sequential CSS schemes for both low and high channel availability in terms of average throughput and overhead. We also notice that the average opportunistic throughput for all schemes increases while the average sensing overhead decreases when the channel availability $P_{I}$ becomes larger. This is due to the fact that when the channel availability is larger, there are more opportunities to find vacant channels in a fixed time period which leads to higher opportunistic throughput and lower sensing overhead.

Figure 3.22 shows the throughput efficiency versus the SNR with parameters $|G|=4$ 


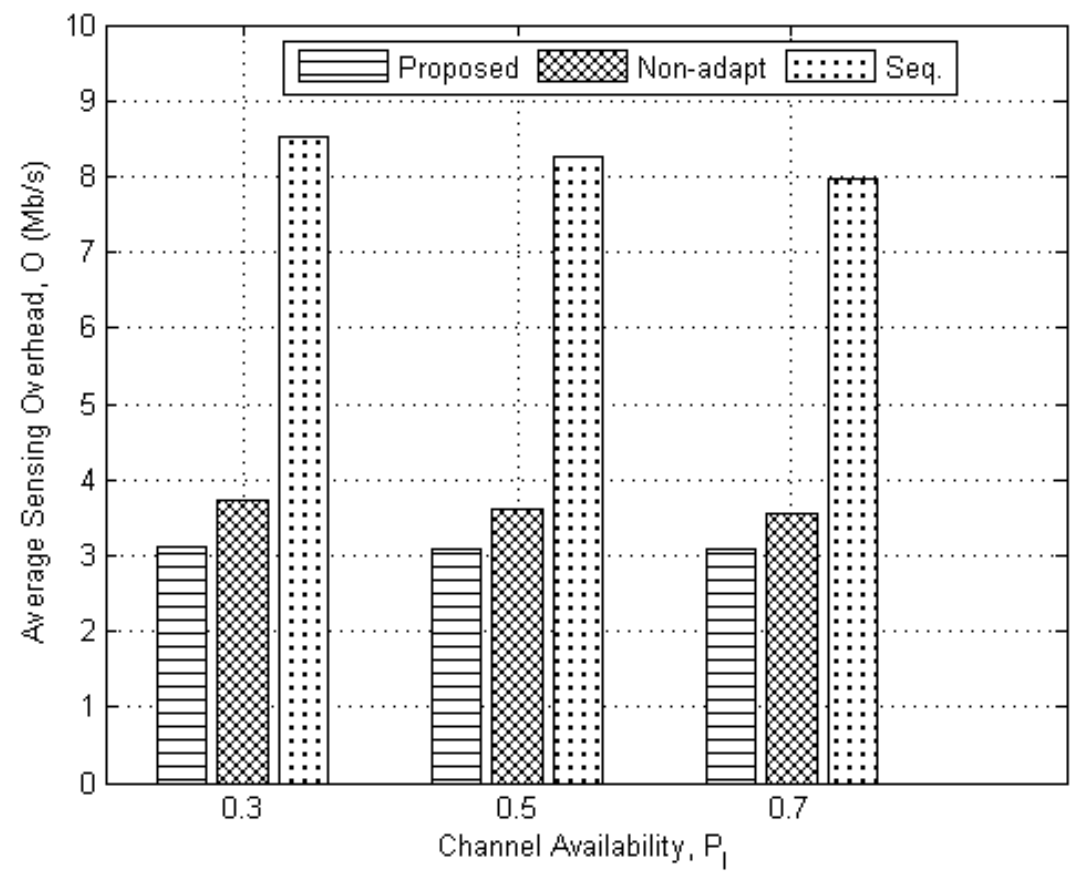

Figure 3.21: Average sensing overhead versus channel availability for $|G|=4$ and $K=6$.

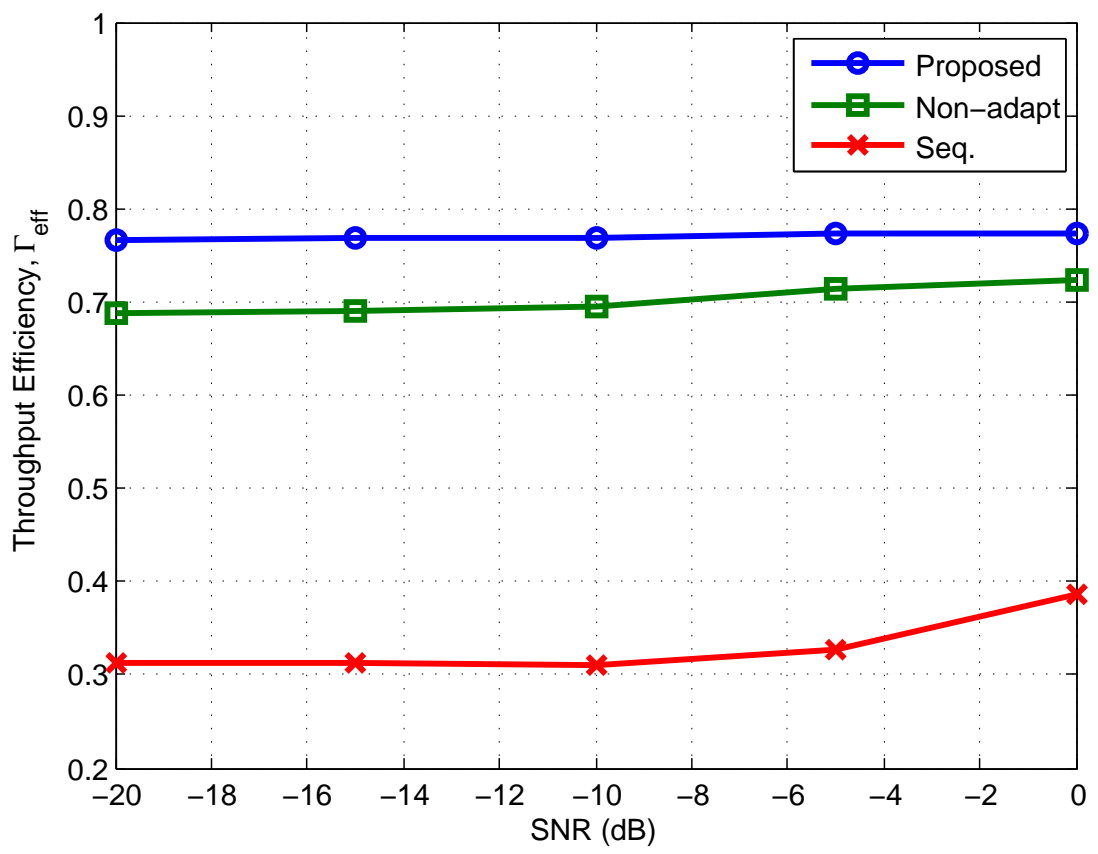

Figure 3.22: Throughput efficiency versus SNR for $|G|=4$ and $K=6$. 
and $K=6$ for the three schemes considered. We observe from the figure that the proposed algorithm outperforms the non-adaptive and sequential CSS schemes for the different SNR considered. We also observe that better performance can be achieved for all three schemes when SNR increases. Better SNR results in lower sensing errors which results in performance improvement in terms of average opportunistic throughput and average sensing overhead.

\subsection{Chapter Summary}

In this chapter, we focused on two important design criteria for coperative spectrum sensing which are the sensing accuracy and sensing efficiency. We considered an adaptive groupbased cooperative sensing scheme where the secondary users have heterogeneous sensing abilities. We aimed to maximize the throughput efficiency that takes into account both the average opportunistic throughput and the average sensing overhead without degrading the sensing accuracy. We derived the average opportunistic throughput and average sensing overhead for both cases of fixed and variable secondary transmission rates. We formulated the throughput efficiency maximization problem as a non-linear binary programming problem and proposed an efficient heuristic adaptive assignment algorithm to solve the formulated problem. The proposed algorithm adaptively performs user-group and group-sensing round assignments to minimize the number of sensing rounds and the number of secondary users needed to discover an available channel and thus increasing the throughput efficiency while satisfying a predefined limit on the sensing accuracy. The proposed algorithm has a low computational complexity and its performance is within $4.2 \%$ and $2.75 \%$ of the optimal exhaustive search for the fixed and variable transmission rates cases, respectively, when $|G|=2$. For the variable transmission rate case, simulation results demonstrated that the proposed algorithm can provide improvement in the throughput efficiency for up to $19 \%$ and $86 \%$ when compared to non-adaptive grouping and sequential cooperative sensing schemes, respectively, for $|G|=6$ and $K=12$. We examined the effect of different parameters such as 
the number of cooperating users and channels, channel availability and different values of the signal-to-noise-ratio on the proposed assignment algorithm. The proposed algorithm was consistently able to outperform the non-adaptive and sequential CSS schemes for the different parameters examined for both cases of fixed and variable secondary transmission rates.

In this chapter, our goal was to design an efficient adaptive user-group assignment algorithm and evaluate its performance for a given combining scheme used at the fusion center. In our performance evaluation, we assumed that the performance of each secondary user detector, in terms of the probabilities of detection and false alarm, is already available at the fusion center. In the next chapter, we do not assume any knowledge of the performance of each secondary user detector, but use the fusion center past decisions and the current local decisions reported by secondary users to estimate the probabilities of detection and false alarm for each secondary user. Using the estimated local probabilities of false alarm and detection for each detector, we propose a reliability-based decision fusion scheme that assigns a weight to each secondary user before combining the local decisions of the secondary users at the fusion center. 


\section{Chapter 4}

\section{Reliability-based Decision Fusion}

\section{Scheme for CSS Based on Past}

\section{Decisions}

\subsection{Introduction}

The performance of spectrum sensing depends on the employed fusion scheme when cooperative spectrum sensing (CSS) is used. To combine the sensing information from multiple secondary users, either hard combining-based (decision) fusion [49] or soft combining-based (data) fusion schemes [37] can be used. For a decision fusion scheme, each secondary user performs local spectrum sensing independently and then makes a binary decision, and forwards this decision to a fusion center which makes the final decision. For a data fusion scheme, each secondary user sends its observation value (test statistic) directly to the fusion center for it to make the final decision. In conventional decision fusion schemes, such as OR and AND fusion rules, the fusion center combines the binary local decisions reported by the secondary users without taking into account the difference in local sensing reliability between secondary users. In this chapter, we propose a novel reliability-based cooperative 
decision fusion scheme which considers the reliability of the secondary users' local decisions when making a final decision at the fusion center. We assign different weights to secondary users' decisions based on the reliability of their decisions. We use past information about the local and global decisions to estimate the reliability of the sensing decision obtained from each secondary user. We formulate the problem of minimizing the probability of sensing error at the fusion center, subject to a limit on the network probability of detection, as a constrained non-linear integer programming problem. Since the formulated problem is generally NP-hard, we use a non-linear Lagrangian approach, in which the constrained problem is reduced to an unconstrained problem using a non-linear exponential Lagrangian function [94]. We then develop a dual search algorithm to solve the formulated optimization problem. Simulation results show that the proposed solution can achieve optimal results with zero duality gap using only few number of iterations. We compare the performance of the proposed reliability-based fusion scheme, in terms of the minimum probability of sensing error, with the OR and AND fusion schemes. For the group-based CSS considered in Chapter 3, the proposed reliability-based fusion scheme can be employed to combine the decisions of the set of secondary users in each group instead of the OR fusion rule to further enhance the performance of CSS.

\subsection{Related Work}

For the decision fusion schemes, the authors in [95] proposed a decision fusion rule for the sensing-throughput trade-off design that considers the differences in the signal-to-noise ratios (SNR) of the secondary users. These differences were reflected in the weighing of the decisions based on the likelihood ratio test at the fusion center. Another decision fusion scheme was proposed in [96] that combines all secondary users decisions, taking into account the credibility of each decision via Dempster-Shafer (D-S) theory of evidence. DempsterShafer theory is an alternative model to the traditional Bayesian probabilistic theory for 
the mathematical representation of uncertainty. It offers a way to combine evidence from multiple observers without the need to know about prior or conditional probabilities as in the likelihood ratio test approaches. However, it needs to determine the initial estimates of secondary users' trustworthiness. This scheme can give a significant improvement in detection probability as well as reduction in false alarm rate, and is best suited for a fastchanging radio frequency environment. In [97], an enhanced scheme was proposed that assigns a reliability value to each detector based on its SNR. This value reflects the relative relationship between detectors and is used to adjust the credibility of each decision to a more accurate value before combining the decisions via D-S theory. In [98], a centralized trust management scheme for secondary users' base station was proposed. The authors introduced the notion of self-confidence and trust. Self-confidence is a rate supplied by a sensing secondary user of its own confidence on the accuracy of its sensing results. Trust is a measure of reputation, and represents the historical accuracy of the secondary user's sensing reports. The authors incorporated the trustworthiness evaluation from a modified Beta reputation model into the $K$-out-of- $M$ decision fusion rule to give greater weight to the opinions of more trustworthy secondary users. In [99], the authors proposed a trust weighted cooperative spectrum sensing scheme to identify malicious secondary users and mitigate their harmful effect on sensing performance. To make an accurate final decision, the trust weight factor of each SU was calculated by their trust values. The fusion center initializes the trust values of SUs by the number of correct sensing decisions that agrees with the primary user's actual behaviour and the number of total sensing. The trust value for each $\mathrm{SU}$ is then increased or decreased according to whether it provides correct or false sensing information. Simulation results showed a performance improvement in the probability of detection as compared to the conventional CSS scheme without trust weighted factors for the OR, AND and majority voting fusion rules.

For the data fusion schemes, the authors in [100] proposed a cooperation strategy in which the local decisions were combined with weighting factors that reflected the local sensing 
reliability of each secondary user based on partial channel state information to make a final decision with the correspondingly optimized threshold level. In [101], the authors proposed a weighted CSS scheme for which the optimal weights were derived under the constraint of equal probabilities of false alarm and miss-detection. They also incorporated reference matrix into the weight setting procedure to store the most recent sensing data in either a noise matrix or a signal energy matrix according to their corresponding sensing decisions in order to acquire the primary user's signal energies from the sensing data of the cooperating secondary users. In [102], the authors proposed a CSS technique which considers the spatial variation of secondary users and each user's contribution was weighted by a factor that depends on the received power and path loss. The proposed scheme provided better probability of detection and spectrum utilization when compared to the equal gain combining scheme.

In [103], the authors proposed a weighted CSS scheme that assigns weights to secondary users based on the local detection accuracy of each secondary user, instead of the received SNR. In this scheme, the authors used the total probability of error, which combines the false alarm probability and miss-detection probability, to measure the detection accuracy. At the fusion center, each cooperating user was assigned a weight corresponding to its probability of error, i.e., a SU with higher probability of error was assigned lower weight. The optimal detection threshold, as well as the number of SUs required to participate in cooperative sensing were derived, subject to a given total probability of error. Simulation results showed that the proposed scheme provides performance improvement, in terms of the probability of error, when compared to the equal weighted and SNR-based weighted schemes. The authors in [104] proposed a penalty-based weight adjustment mechanism for CSS to enhance the adaptability of secondary users in time-varying environments. Similar to [103], each secondary user was characterized by its probability of error but the weight factor was adjusted using a penalty mechanism based on the current local decision made by each secondary user. The final result was then computed by fusing the weighted soft decision made by each cooperating secondary user. 
In [105], an adaptive weighting scheme with double threshold energy detection based on the water-filling principle was proposed for cooperative spectrum sensing. For this scheme, each secondary user was allocated a weighting factor based on the relation between the instantaneous SNR of the sensing channel and the water level. The authors showed that the proposed weighting scheme can achieve better detection performance and lower average number of sensing bits when compared to the equal weighted and SNR weighted schemes. A cooperative spectrum sensing scheme based on ROCQ reputation management model for cognitive radio networks was proposed in [106]. The ROCQ scheme is a reputationbased trust management system that computes the trustworthiness of peers on the basis of transaction-based feedback. The ROCQ model combines four parameters: (i) reputation, (ii) a peer's global trust rating or opinion formed by a peer's first-hand interactions, (iii) credibility of a reporting peer and (iv) the quality or the confidence a reporting peer puts on the feedback it provides. In this scheme, each secondary user has a reputation degree used to calculate its coefficient in the linear fusion process, and the reputation degree is initialized and adjusted by the fusion center according to each secondary user's sensing result, sensing correctness and report consistency. Simulation results showed that the detection performance of the proposed scheme in [106] is approximately the same as that of the optimal linear fusion scheme while it requires no instantaneous SNR.

In the problem of fusing decisions from independent detectors by a fusion center, the probabilities of detection and false alarm must be known. However, in practice, these probabilities may not be known or consistent. In [107], the authors derived an adaptive system to estimate the probabilities of detection and false alarm for each detector in a distributed detection system by a simple counting process. Since the method does not require a training sequence, it is based on reinforcement learning. In [108], a secure distributed spectrum sensing scheme was proposed that uses robust statistics to approximate the distributions for both hypotheses of all nodes based on their past data reports. Achieved parameters were used for testing of malicious users and calculating necessary information for data fusion, 
with a reliability evaluation stage, by means of D-S theory. In [109], the reliability of the local detectors was obtained by estimating the probabilities of detection and false alarm of each detector based on past decisions. The authors showed that the value of the global decision should be chosen carefully to provide a trade-off between the network probabilities of detection and false alarm.

In most of the previous work on cooperative spectrum sensing that considers weighted contribution from each user, the focus was: (i) how to obtain the optimal weight for each user based on some performance criteria by assuming knowledge of the local probabilities of false alarm and detection of each local detector which may not be known in practice, e.g., $[95,100,101]$ or (ii) directly setting the weight for each user by assuming certain knowledge of each user's SNR, which is not easy to obtain, especially in low-SNR regime, and optimizing the detection threshold, e.g., [102, 103, 105].

Contrary to most previous work, we do not assume any knowledge of the performance of each secondary user detector, but use the fusion center results and the local decisions made by secondary users to estimate the probabilities of detection and false alarm for each secondary user. Considering secondary users with heterogeneous sensing ability, we propose a novel reliability-based scheme that assigns the weights based on the estimated local probabilities of false alarm and detection for each secondary detector. The local probabilities are obtained without the need of the knowledge of each user's SNR which makes the proposed reliability-based scheme more practical. Considering the trade-off between the network probabilities of false alarm and detection, our objective is to obtain the optimal global threshold that minimizes the network probability of sensing error. By using this objective, we are considering the sensing errors that occur when the channel is busy and idle while taking the primary user's activity in consideration. 


\subsection{Problem Formulation}

We consider a cognitive radio network with $K$ secondary users which can opportunistically access the licensed spectrum allocated to primary users using the energy detection technique described in section 3.3.1. We consider the alternating ON/OFF model for the primary user channel usage pattern and the periodic sensing-transmission structure for secondary users as described in section 3.3.2. Figure 4.1 shows a general frame structure with periodic sensing, in which each frame consists of a sensing period and a data transmission period with the proposed fixed observation window.

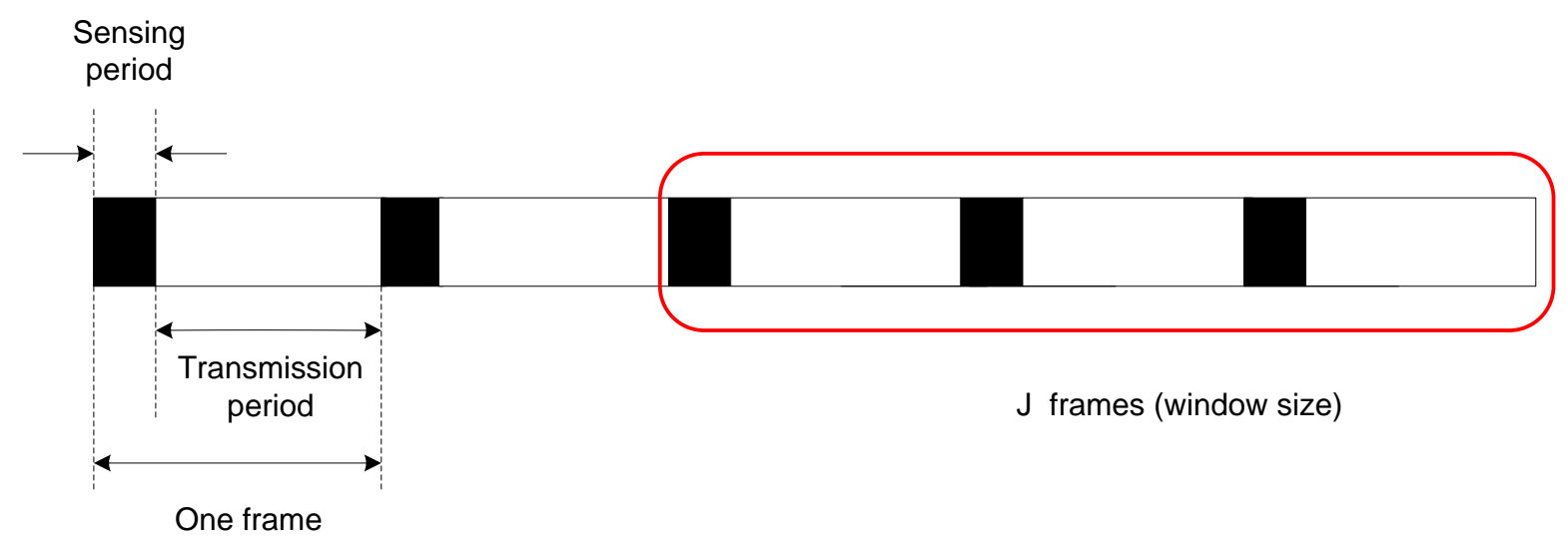

Figure 4.1: Frame structure for secondary users with fixed observation window.

We consider the case in which each individual secondary user makes a decision, $d_{k}$, on the absence or presence of the primary user's transmission based on the local sensing information, such that,

$$
d_{k}= \begin{cases}1, & \text { if } Y_{k}>\gamma_{k} \\ -1, & \text { if } Y_{k} \leq \gamma_{k}\end{cases}
$$

where $\gamma_{k}$ is the threshold of the $k^{t h}$ local detector.

The decisions of the secondary users are then transmitted to the fusion center that makes a final decision, $d_{0}$, on the absence or presence of the primary user's transmission as a result of weighted combination of the secondary users' local decisions as shown in Figure 4.2. The 


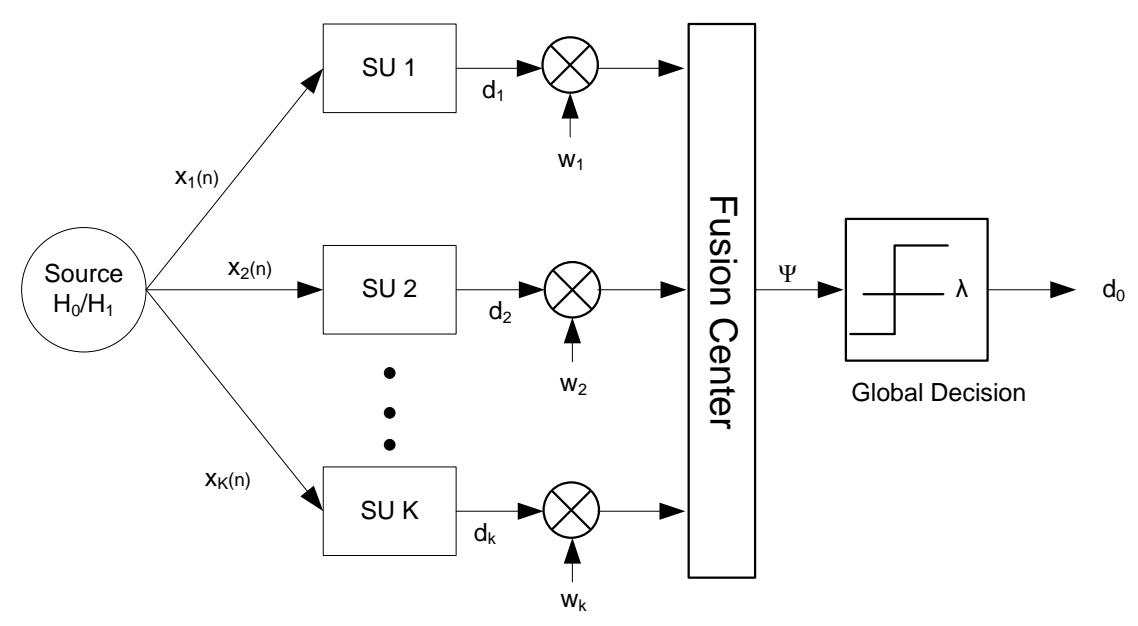

Figure 4.2: Weighted cooperative spectrum sensing scheme.

test statistic for the fusion center is given by

$$
\Psi=\sum_{k=1}^{K} w_{k} d_{k}
$$

where $w_{k}$ is the combining weight of the $k^{t h}$ secondary user and $\lambda$ represents the global threshold level according to which a global decision at the fusion center is made, such that,

$$
d_{0}= \begin{cases}1, & \text { if } \Psi>\lambda \\ -1, & \text { if } \Psi \leq \lambda\end{cases}
$$

To optimize the performance of cooperative spectrum sensing, the global test statistic $\Psi$, which depends on the weighting coefficient $w_{k}$ and the local decision $d_{k}$, needs to be known. According to the local sensing performance, we can represent the local decision of each secondary user $k$ as

$$
d_{k \mid H_{1}}=\left\{\begin{array}{l}
1, \quad \text { with probability } P_{d, k} \\
-1, \quad \text { with probability } 1-P_{d, k}
\end{array}\right.
$$


and

$$
d_{k \mid H_{0}}=\left\{\begin{array}{l}
1, \quad \text { with probability } P_{f, k} \\
-1, \quad \text { with probability } 1-P_{f, k}
\end{array}\right.
$$

where $P_{d, k}$ and $P_{f, k}$ are the local probabilities of detection and false alarm of the $k^{t h}$ secondary user, respectively.

The conditional probability density function (pdf) of the weighted local decision for each user $e_{k} \triangleq w_{k} d_{k}$ under each hypothesis can be represented as,

$$
f_{E_{k}}\left(e_{k} \mid H_{x}\right)= \begin{cases}P_{f, k} \delta\left(e_{k}-w_{k}\right)+\left(1-P_{f, k}\right) \delta\left(e_{k}+w_{k}\right), & x=0 \\ P_{d, k} \delta\left(e_{k}-w_{k}\right)+\left(1-P_{d, k}\right) \delta\left(e_{k}+w_{k}\right), & x=1\end{cases}
$$

The characteristic function of $\Psi$ under each hypothesis, $H_{x}, x \in\{0,1\}$, can be represented as $[110]$

$$
\Phi_{\Psi \mid H_{x}}(\omega)=\prod_{k=1}^{K} \Phi_{E_{k} \mid H_{x}}(\omega),
$$

where $\Phi_{E_{k} \mid H_{x}}(\omega)$ is the characteristic function of weighted local decision of the secondary user $k$ for hypothesis $H_{x}, x \in\{0,1\}$ and can be calculated by using the Fourier transform of the probability density function in (4.6), such that,

$$
\Phi_{E_{k} \mid H_{x}}(\omega)= \begin{cases}P_{f, k} e^{\left(\mathbf{i} \omega w_{k}\right)}+\left(1-P_{f, k}\right) e^{-\left(\mathbf{i} \omega w_{k}\right)}, & x=0 \\ P_{d, k} e^{\left(\mathbf{i} \omega w_{k}\right)}+\left(1-P_{d, k}\right) e^{-\left(\mathbf{i} \omega w_{k}\right)}, & x=1\end{cases}
$$

where $\mathbf{i}=\sqrt{-1}$ is the unit imaginary number.

From (4.7) and (4.8), the conditional probability density function of $\Psi$ under each hypothesis is given by [100],

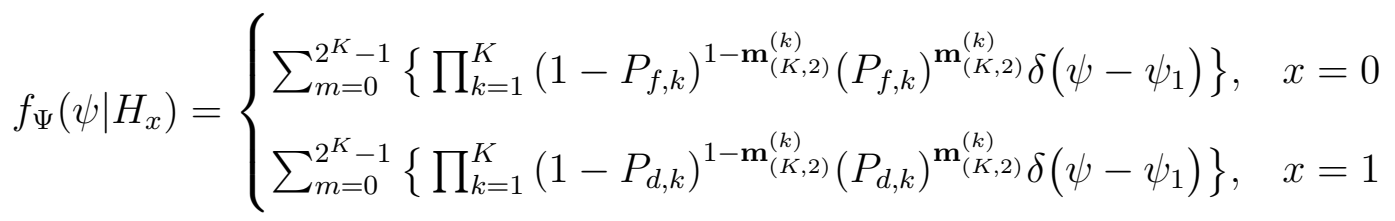


where,

$$
\psi_{1}=\sum_{k=1}^{K} w_{k}\left(2 \mathbf{m}_{(K, 2)}^{(k)}-1\right)
$$

where $\mathbf{m}_{(K, 2)}$ is a $K$-bit binary vector representing the binary transform of the integer number $m \in\left\{0,1, \cdots, 2^{K}-1\right\}$ and $\mathbf{m}_{(K, 2)}^{(k)}$ is the $k^{t h}$ bit of $\mathbf{m}_{(K, 2)}$. For example, if $K=4$, there are 16 possible binary vectors, $\mathbf{m}_{(4,2)}$, with length four (i.e., $\mathbf{0}_{(4,2)}=0000, \mathbf{1}_{(4,2)}=0001, \mathbf{2}_{(4,2)}=$ $\left.0010, \cdots, \mathbf{1 5}_{(4,2)}=1111\right)$. In case of $K=4$ and $m=5, \mathbf{m}_{(K, 2)}=0101, \mathbf{m}_{(K, 2)}^{(1)}=0$, $\mathbf{m}_{(K, 2)}^{(2)}=1, \mathbf{m}_{(K, 2)}^{(3)}=0$ and $\mathbf{m}_{(K, 2)}^{(4)}=1$.

Therefore, the network probability of detection, $P_{D}$, and the network probability of false alarm, $P_{F}$, are respectively given by,

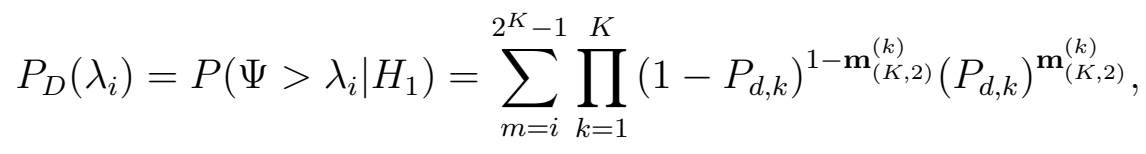

and

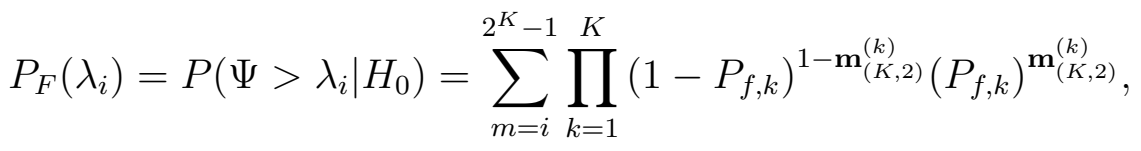

where $i=\left\{0 \cdots 2^{K}-1\right\}$ represents the possible values of $\lambda$ for which $P_{D}$ is greater than zero. The possible value of $\lambda$ corresponding to each $i$, such that, $m=i$, must satisfy the following condition:

$$
\sum_{k=1}^{K} w_{k}\left(2(\mathbf{m}-\mathbf{1})_{(K, 2)}^{(k)}-1\right)<\lambda_{i}<\sum_{k=1}^{K} w_{k}\left(2 \mathbf{m}_{(K, 2)}^{(k)}-1\right)
$$

For a given combining weight, our objective is to obtain the global threshold level $\lambda$, that minimizes the probability of sensing error at the fusion center while maintaining the detection probability at a desired level. Using this sensing objective, we are considering the sensing errors that occur when the channel is busy and idle, i.e, $P_{F}$ and $1-P_{D}$. 
Therefore, we formulate the optimization problem as

$$
\begin{gathered}
\underset{i}{\arg \min } P_{E}\left(\lambda_{i}\right)=\left(1-P_{D}\left(\lambda_{i}\right)\right) P_{B}+P_{F}\left(\lambda_{i}\right) P_{I} \\
\text { s. t. } \quad P_{D}\left(\lambda_{i}\right) \geq \beta \\
\quad i \in\left\{0, \ldots, 2^{K}-1\right\},
\end{gathered}
$$

where $P_{E}$ is the network probability of sensing error. The terms $P_{B}$ and $P_{I}$ are the prior probabilities that the primary user is present and absent on the channel, respectively. The network probabilities of detection, $P_{D}$, and false alarm, $P_{F}$, are given in (4.11) and (4.12), respectively. The limit on the total $P_{D}, \beta$, is used to guarantee a satisfactory level of protection for the primary users. The optimization problem in $\left(P_{1}\right)$ is a constrained nonlinear integer programming problem which is generally $N P$-hard [91] (see Appendix A.2 for further details).

Note that, if all users have equal assigned weights not based on their decision reliability, we can combine them using the conventional decision fusion rules such as OR or AND fusion rules. For the OR fusion rule, all the secondary users have equal weights and the fusion center decides that the primary user is not transmitting only if all secondary users decide the absence of the primary user's signal and the network probabilities of detection, $P_{D}$, and false alarm, $P_{F}$, are given by (2.14) and (2.15), respectively. For the AND fusion rule, if all local detectors decide that there is a primary user, then the final decision at the fusion center declares that there is a primary user and the network probabilities of detection and false alarm are given by (2.16) and (2.17), respectively.

\subsection{Probability Estimation using Past Decisions}

To estimate the local probabilities of detection and false alarm for each secondary user based on past performance, we consider a window of past local and global decisions of size $J$ as shown in Figure 4.1. Since the global decision is more accurate than local decisions [12], 
we use it as a reference and consider the decision of the secondary user in a certain sensing frame, $j$, to be correct if it is in agreement with the global decision made at this sensing interval. For the $j^{\text {th }}$ frame and the $k^{\text {th }}$ secondary user, we introduce an indicator function, $Z_{x, y}^{k, j}$, which is equal to 1 if the local detector decision is $H_{x}$ when the global decision is $H_{y}$, $x, y \in\{0,1\}$, and 0 , otherwise. For $x=y=1$, we define the indicator function as,

$$
Z_{1,1}^{k, j}=\frac{\left(1+d_{k, j}^{\prime}\right)\left(1+d_{0, j}^{\prime}\right)}{4}
$$

where $d_{k, j}^{\prime}$ and $d_{0, j}^{\prime}$ are the decisions made by the $k^{\text {th }}$ secondary user and the fusion center, respectively, at the $j^{\text {th }}$ frame of the observation window $J$.

The values of the indictor function for the $k^{\text {th }}$ user at the $j^{\text {th }}$ frame when both the local and global decisions are $H_{1}(x=y=1), Z_{1,1}^{k, j}$, is shown in Table 4.1.

Table 4.1: Values of the Indictor Function $Z_{1,1}^{k, j}$.

\begin{tabular}{|c|c|c|}
\hline$d_{0, j}^{\prime}$ & $d_{k, j}^{\prime}$ & $Z_{1,1}^{k, j}$ \\
\hline 1 & 1 & 1 \\
\hline 1 & -1 & 0 \\
\hline-1 & 1 & 0 \\
\hline-1 & -1 & 0 \\
\hline
\end{tabular}

For the $k^{\text {th }}$ secondary user, we count the number of times in a certain window of length $J, Z_{x, y}^{k}$, for which the local detector decision is $H_{x}$ when the global decision is $H_{y}$ and $x, y \in\{0,1\}$ as

$$
Z_{1,1}^{k}=\sum_{j=n-J+1}^{n} Z_{1,1}^{k, j}
$$

Similarly,

$$
\begin{aligned}
& Z_{0,1}^{k}=\sum_{j=n-J+1}^{n} Z_{0,1}^{k, j}=\sum_{j=n-J+1}^{n} \frac{\left(1-d_{k, j}^{\prime}\right)\left(1+d_{0, j}^{\prime}\right)}{4}, \\
& Z_{1,0}^{k}=\sum_{j=n-J+1}^{n} Z_{1,0}^{k, j}=\sum_{j=n-J+1}^{n} \frac{\left(1+d_{k, j}^{\prime}\right)\left(1-d_{0, j}^{\prime}\right)}{4},
\end{aligned}
$$




$$
Z_{0,0}^{k}=\sum_{j=n-J+1}^{n} Z_{0,0}^{k, j}=\sum_{j=n-J+1}^{n} \frac{\left(1-d_{k, j}^{\prime}\right)\left(1-d_{0, j}^{\prime}\right)}{4}
$$

and

$$
Z_{0,0}^{k}+Z_{1,1}^{k}+Z_{0,1}^{k}+Z_{1,0}^{k}=J
$$

Since we do not assume any knowledge of the probability density functions of the secondary users' observations, we approximate the local probabilities of detection and false alarm by their relative frequencies [107]. Therefore, we calculate the probabilities of detection and false alarm, respectively, as

$$
P_{d, k}=\frac{Z_{1,1}^{k}}{Z_{0,1}^{k}+Z_{1,1}^{k}}
$$

and

$$
P_{f, k}=\frac{Z_{1,0}^{k}}{Z_{1,0}^{k}+Z_{0,0}^{k}}
$$

\subsection{Proposed Reliability-based Decision Fusion Scheme}

We consider secondary users with heterogeneous sensing ability in terms of the local probabilities of detection and false alarm. Therefore, different secondary users will have different local decision reliability. Since it is more efficient to take into account the differences in the reliability of the decisions made by different secondary users, we assign a different weight to each secondary user's local decision. In the proposed scheme, the combining weight given for each secondary user's local decision reflects the reliability of this user's past decisions. It is evident that the secondary user with higher detection probability and lower false alarm probability has a higher reliability and, therefore, should contribute more to the final decision made by the fusion center. Therefore, we define the reliability of each secondary user as,

$$
r_{k}=P_{d, k}\left(1-P_{f, k}\right)
$$


and we obtain the weight of the $k^{\text {th }}$ secondary user by,

$$
w_{k}=\frac{r_{k}}{\sum_{k=1}^{K} r_{k}},
$$

such that $\sum_{k=1}^{K} w_{k}=1$.

Using the approximate values of $P_{d, k}$ and $P_{f, k}$ calculated using (4.20) and (4.21), respectively, we can estimate the reliability of the $k^{\text {th }}$ secondary user and use it to find the corresponding combining weight.

Our proposed reliability-based decision fusion scheme can be summarized as follows:

Step 1 : Compare the past local decisions and global decisions in a window of size $J$, and obtain the approximate values of $P_{d, k}$ and $P_{f, k}$ from (4.20) and (4.21), respectively.

Step 2 : Using the approximate values of $P_{d, k}$ and $P_{f, k}$, estimate the reliability of the $k^{\text {th }}$ secondary user from (4.22) and use it to find the combining weight, $w_{k}$, for the $k^{\text {th }}$ secondary user using (4.23).

Step 3 : For a given target probability of detection, $\beta$, obtain the optimum global threshold index, $i^{\text {opt }}$, that minimizes the probability of sensing error in $\left(P_{1}\right)$.

Step 4 : Using the obtained global threshold index, $i^{\text {opt }}$, and using the combining weight obtained for each user, find the optimum global threshold, $\lambda^{o p t}$, satisfying (4.13).

Step 5 : The final decision of the fusion center is made by comparing the test statistics in (4.2) to the optimum global threshold, $\lambda^{\text {opt }}$.

\subsection{Dual Search Algorithm}

To solve the problem of minimizing the network probability of sensing error, we adopt the generalized non-linear Lagrangian relaxation method in [94]. Similar to the conventional linear Lagrangian theory, the constrained problem is reduced to an unconstrained problem 
using a non-linear Lagrangian function which is a non-linear function of the cost and constraint functions. The key concept in introducing non-linear Lagrangian formulations is the construction of a non-linear support of the perturbation function at the optimal point while the linear support associated with the traditional linear Lagrangian may fail to exist. While possessing an asymptotic strong duality property, the non-linear Lagrangian formulations offer a success guarantee for the identification of an optimal primal solution via a dual search $[111,112]$.

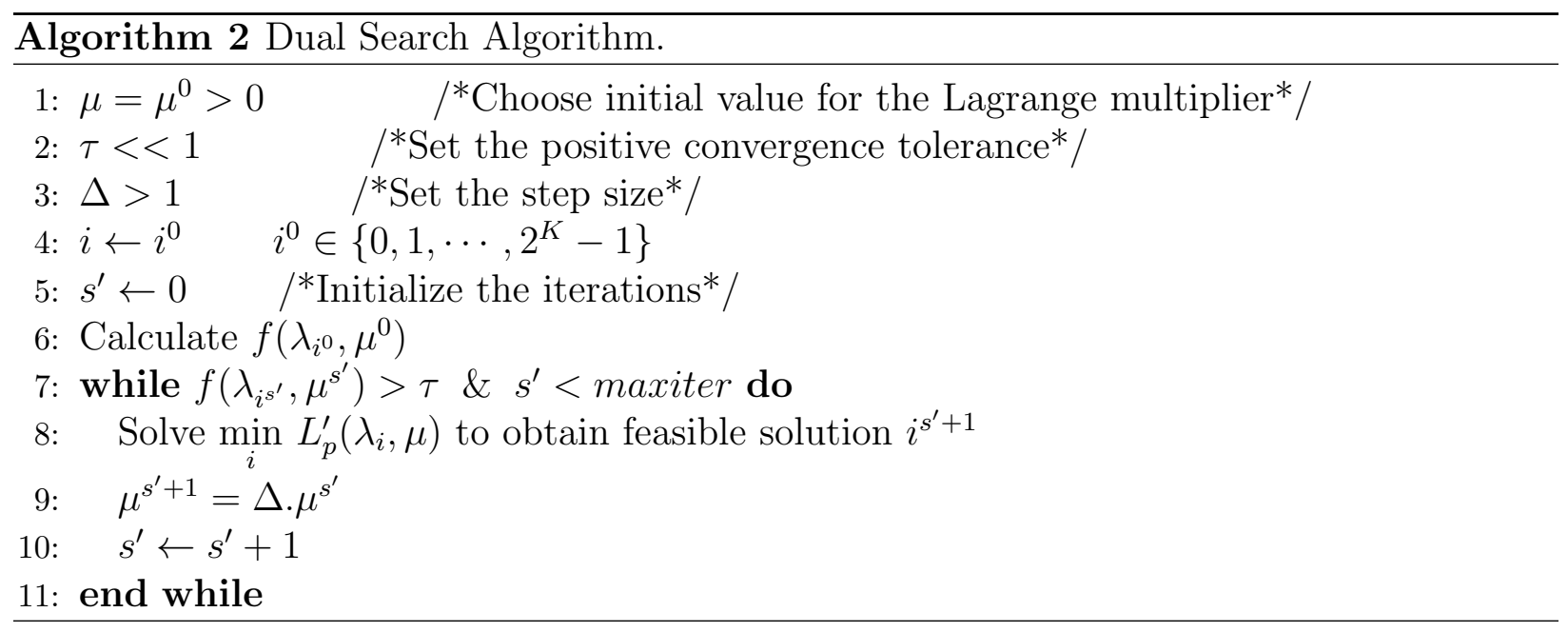

In this thesis, we consider the exponential generalized Lagrangian function (GLF) proposed for bounded integer programming in [94,112]. Therefore, for some given $p>0$,

$$
\begin{aligned}
L_{p}^{\prime}\left(\lambda_{i}, \mu\right) & =P_{E}\left(\lambda_{i}\right)+\frac{1}{\mu} \exp \left(\mu\left(\beta-P_{D}\left(\lambda_{i}\right)\right)\right) \\
& =P_{E}\left(\lambda_{i}\right)+f\left(\lambda_{i}, \mu\right) \quad \mu \geq p>0 .
\end{aligned}
$$

We define the GLF-based Lagrangian relaxation associated with the problem $\left(P_{1}\right)$ as

$$
\phi_{p}(\mu)=\min _{i} L_{p}^{\prime}\left(\lambda_{i}, \mu\right)
$$


And the dual problem associated with (P1) is formulated as

$$
\theta_{p}(\mu)=\max _{\mu>0} \phi_{p}(\mu)
$$

It was shown in [94] that the duality gap, which is the difference between the optimal solution of the primal problem and dual problem, is bounded by $1 / p$ for finite $p$. Therefore,

once the parameter $p$ exceeds a threshold, an optimal solution of primal problem (P1) can be obtained using the non-linear exponential Lagrangian formulation. Another property of the dual function for the exponential generalized Lagrangian function, $\theta_{p}(\mu)$, is that it is unimodal. This property guarantees that the local maximum of the dual function is also a global maximum which facilitates the dual search.

Based on minimizing the non-linear Lagrangian function, $f\left(\lambda_{i}, \mu\right)$, we construct the dual algorithm presented in Algorithm 2 to obtain the optimal solution $i^{\text {opt }}$ (Step 3) for the primal problem (P1).

\subsection{Performance Evaluation}

In this section, we evaluate the performance of the proposed scheme using Monte Carlo simulations and the results are obtained by averaging over 1000 simulation runs. We assume that the secondary detectors have equal local thresholds, i.e. $\gamma_{k}=\gamma, \forall k$ but each secondary user has a different SNR, unless otherwise specified, which is assumed to be constant during the observation window, $J$. The probability that the primary user is absent $P_{I}=0.3$, and the probability that the primary user is present $P_{B}=0.7$. We estimate the local probabilities of false alarm and detection for each user at each simulation run using (4.20)-(4.21) and average over 1000 runs to obtain the final estimate for $P_{d, k}$ and $P_{f, k}$. 


\subsubsection{Simulation Results}

First, we examine the effect of different parameters such as the number of users, window size and the signal-to-noise ratio on the performance of the proposed reliability-based fusion scheme. We numerically evaluate the network probabilities of detection, false alarm and sensing error with different window sizes $J$ and different global thresholds, $\lambda$. Figures 4.3 and 4.4 show the network probabilities of detection, $P_{D}$ and false alarm, $P_{F}$, respectively, versus the window size for different values of the global threshold, $\lambda$, when the number of secondary users $K=4$. We can see from the figures that, for a certain $\lambda, P_{D}$ and $P_{F}$ will be overestimated when the window size is small. However, as the window size increases, the variations in $P_{D}$ and $P_{F}$ start to decrease. After a certain window size, that is different for different values of $\lambda$, we can achieve a good estimate of $P_{D}$ and $P_{F}$ with small error. From the figures, we also notice that, decreasing $\lambda$ results in higher probabilities of detection and false alarm.

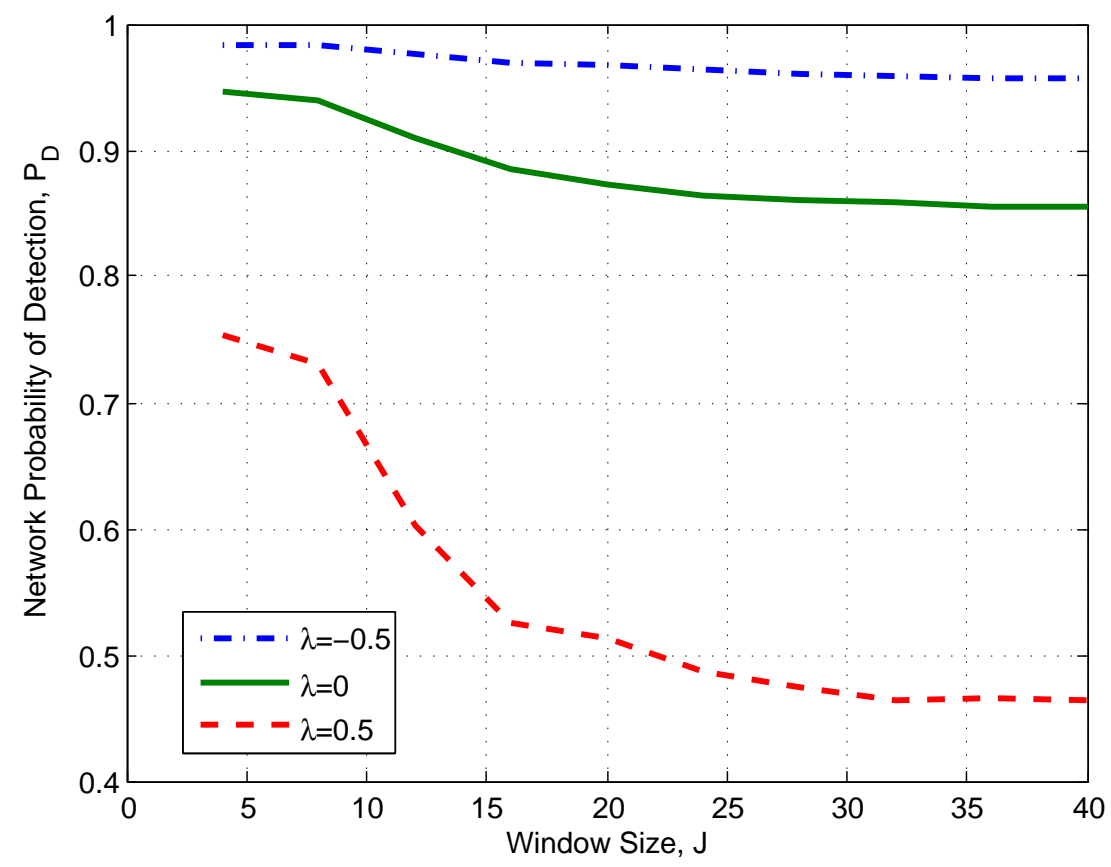

Figure 4.3: Network probability of detection versus the window size for different values of the global threshold, $\lambda$, for $K=4$. 


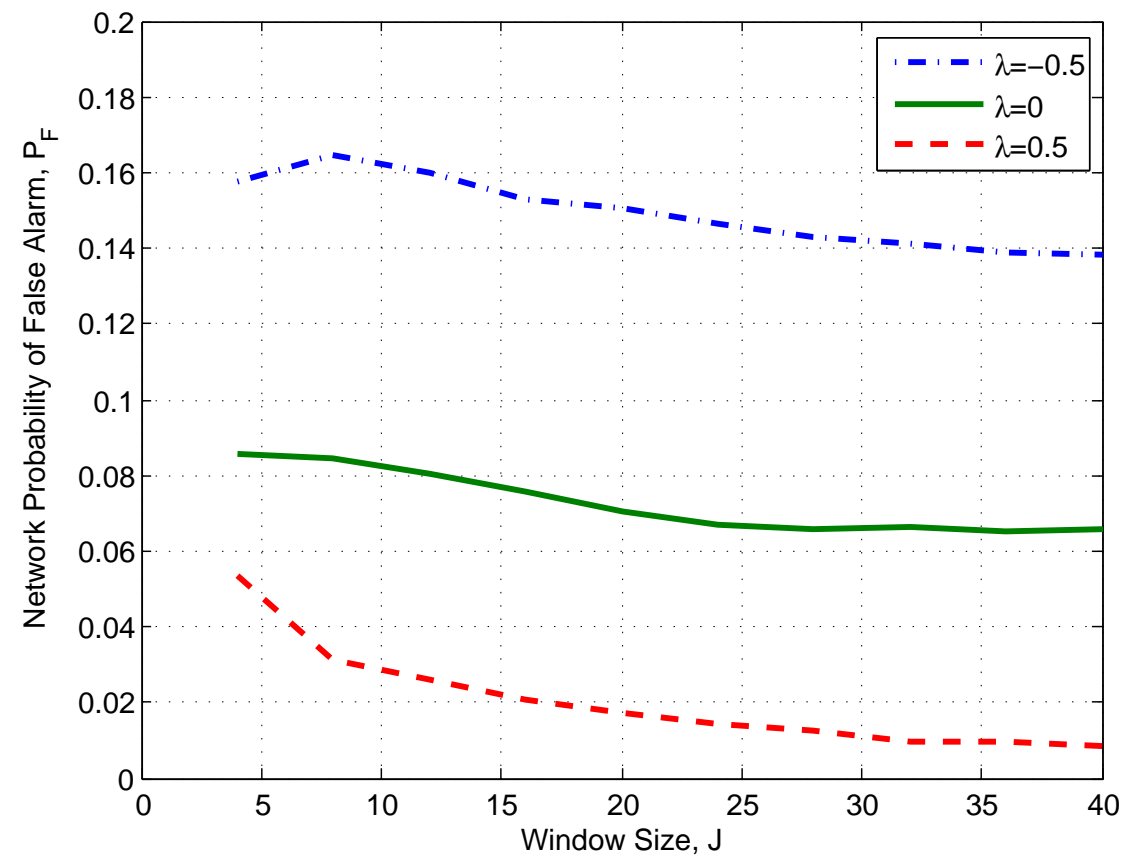

Figure 4.4: Network probability of false alarm versus the window size for different values of the global threshold, $\lambda$, for $K=4$.

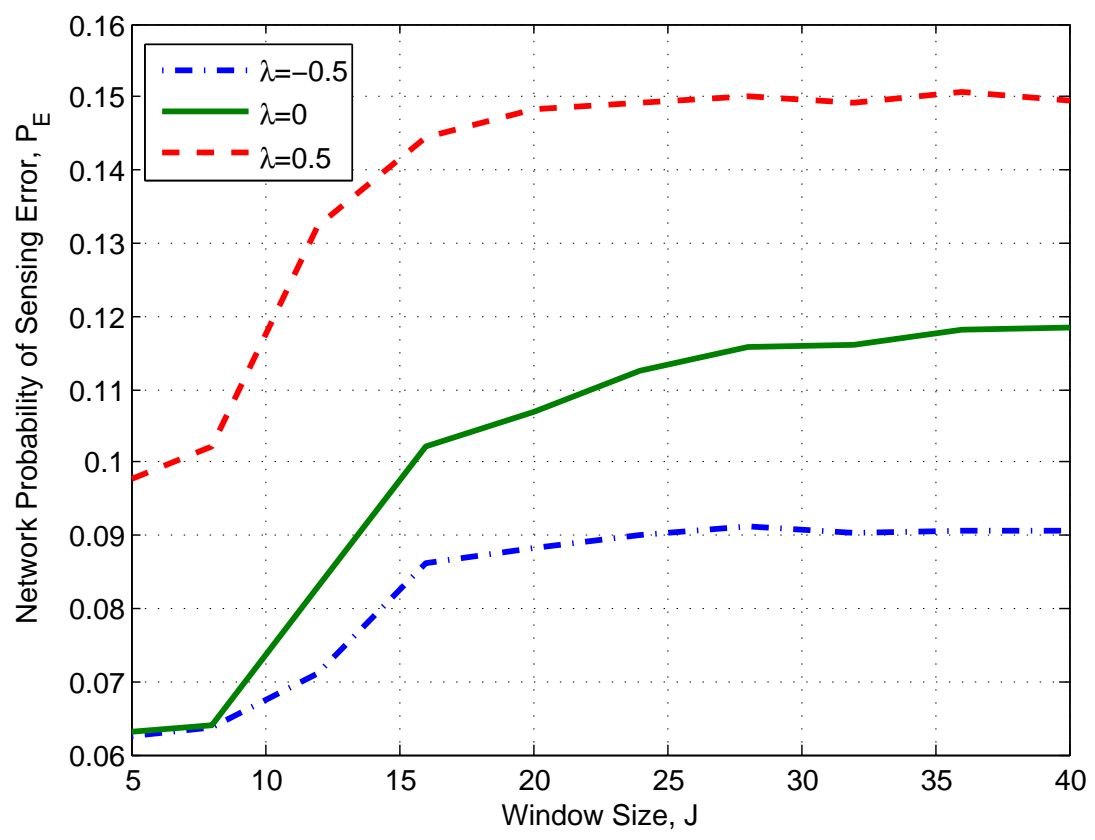

Figure 4.5: Network probability of sensing error versus the window size for different values of the global threshold, $\lambda$, for $K=4$. 
In Figure 4.5, we plot the network probability of sensing error, $P_{E}$, versus the window size for different values of the global threshold, $\lambda$, when the number of secondary users $K=4$. We can see from the figure that, for a certain $\lambda, P_{E}$ will be underestimated when the window size is small. However, after a certain window size, that is different for different values of $\lambda$, the variation in $P_{E}$ decreases and we can have a good estimate of $P_{E}$ with small error. We also notice from the figure that a lower probability of sensing error can be achieved by decreasing the global threshold, $\lambda$.

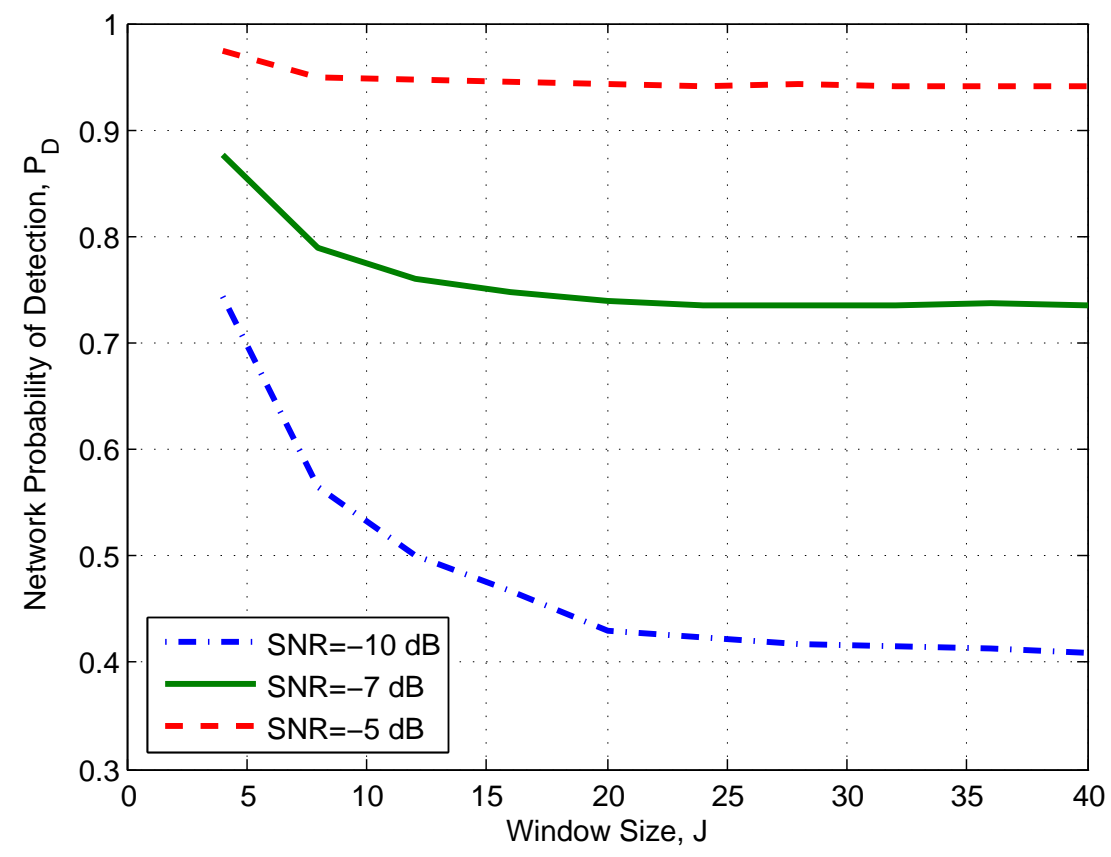

Figure 4.6: Network probability of detection versus the window size for different values of the SNR for $\lambda=0$ and $K=4$.

To show the effect of the SNR on the network probabilities of detection and false alarm with the different window sizes, we assume that all users have the same average SNR and we set the global threshold to be $\lambda=0$. Figures 4.6 and 4.7 show the network probability of detection, $P_{D}$, and the network probability of false alarm, $P_{F}$, versus the window size for different values of the SNR with $K=4$, respectively. As we can see from the figures, $P_{D}$ and $P_{F}$ will be overestimated when the window size is small especially for lower values of SNR. As the window size increases, a good estimate of $P_{D}$ and $P_{F}$ can be obtained. The estimated 


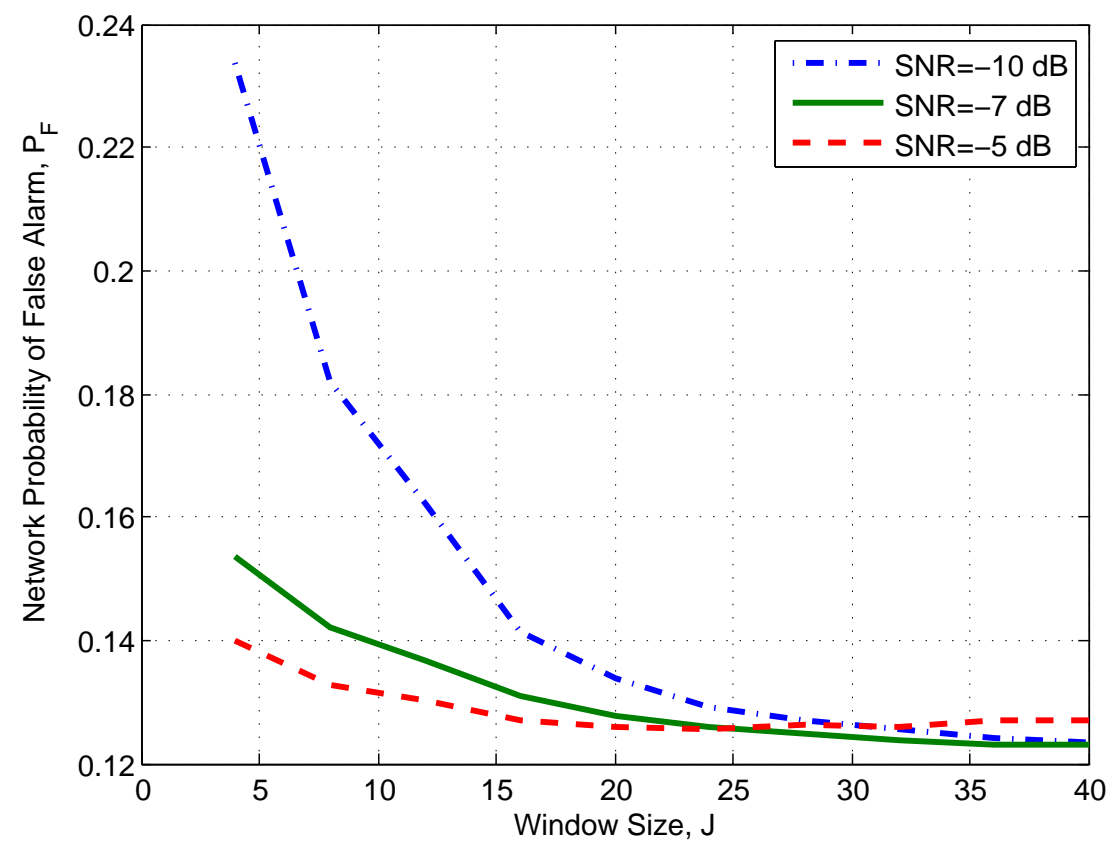

Figure 4.7: Network probability of false alarm versus the window size for different values of the SNR for $\lambda=0$ and $K=4$.

value of $P_{F}$ will not be affected by the differences in SNR since it does not depend on it. However, as the SNR increases, higher $P_{D}$ can be achieved for the same value of $P_{F}$.

In Figure 4.8, we compare the performance of the proposed scheme with that of the OR, AND fusion schemes using the receiver operating characteristic curves which shows the relationship between $P_{D}$ and $P_{F}$. For this comparison, the global threshold, $\lambda$, is set to 0 and the window size is set to be $J=25$ since as observed from the previous figure, there is no significant performance improvement obtained by increasing the window size beyond this value. Considering secondary users with different SNR, the SNR vector is set to $\Gamma^{\prime}=[-20 \mathrm{~dB}$ $-12 \mathrm{~dB}-9.5 \mathrm{~dB}-7.9 \mathrm{~dB}]$ for $K=4$. From the figure, we can see that for the same probability of false alarm, $P_{F}$, the proposed scheme provides higher probability of detection, $P_{D}$ when compared to OR and AND fusion schemes. For example, for $P_{F}=0.1$, the proposed scheme offers approximately $12 \%$ and $25 \%$ improvement in $P_{D}$ over the OR scheme and AND fusion schemes, respectively.

Figure 4.9 shows the network probability of sensing error versus the global threshold 


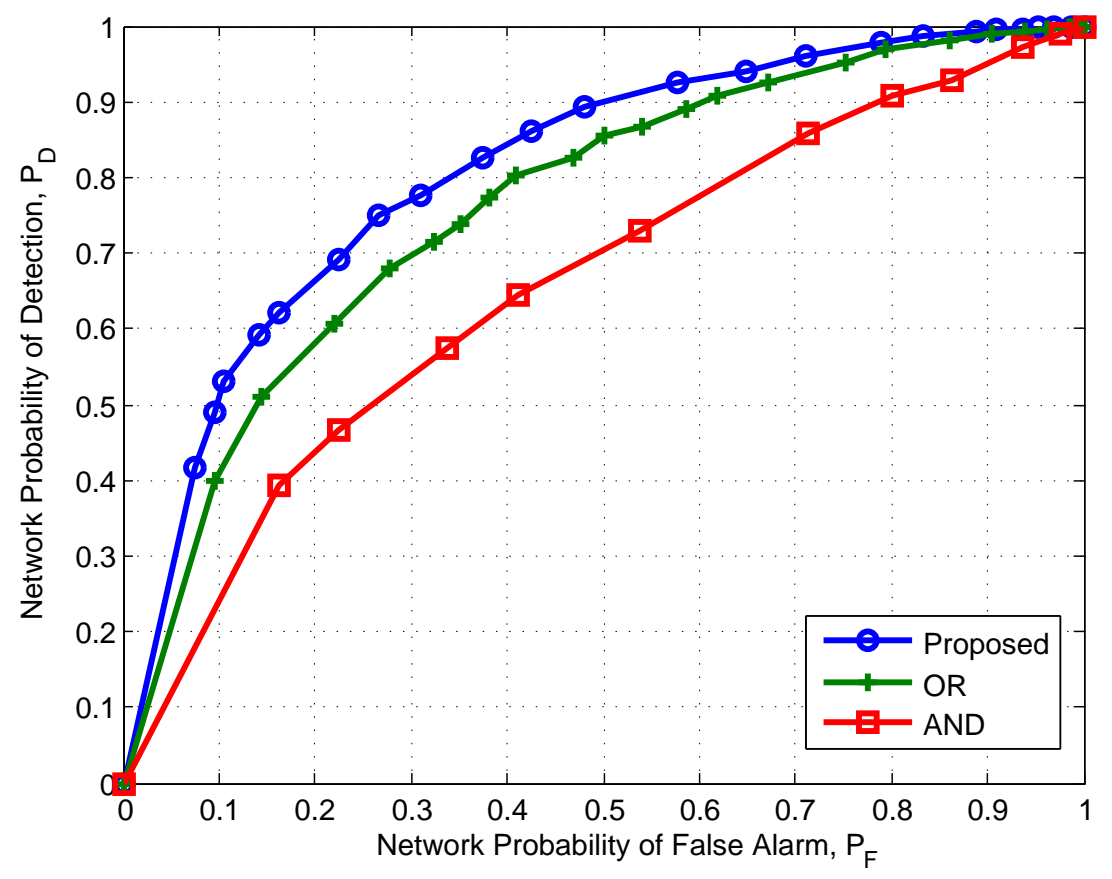

Figure 4.8: Comparison of the receiver operating characteristic curves for the proposed scheme and the OR and AND fusion schemes.

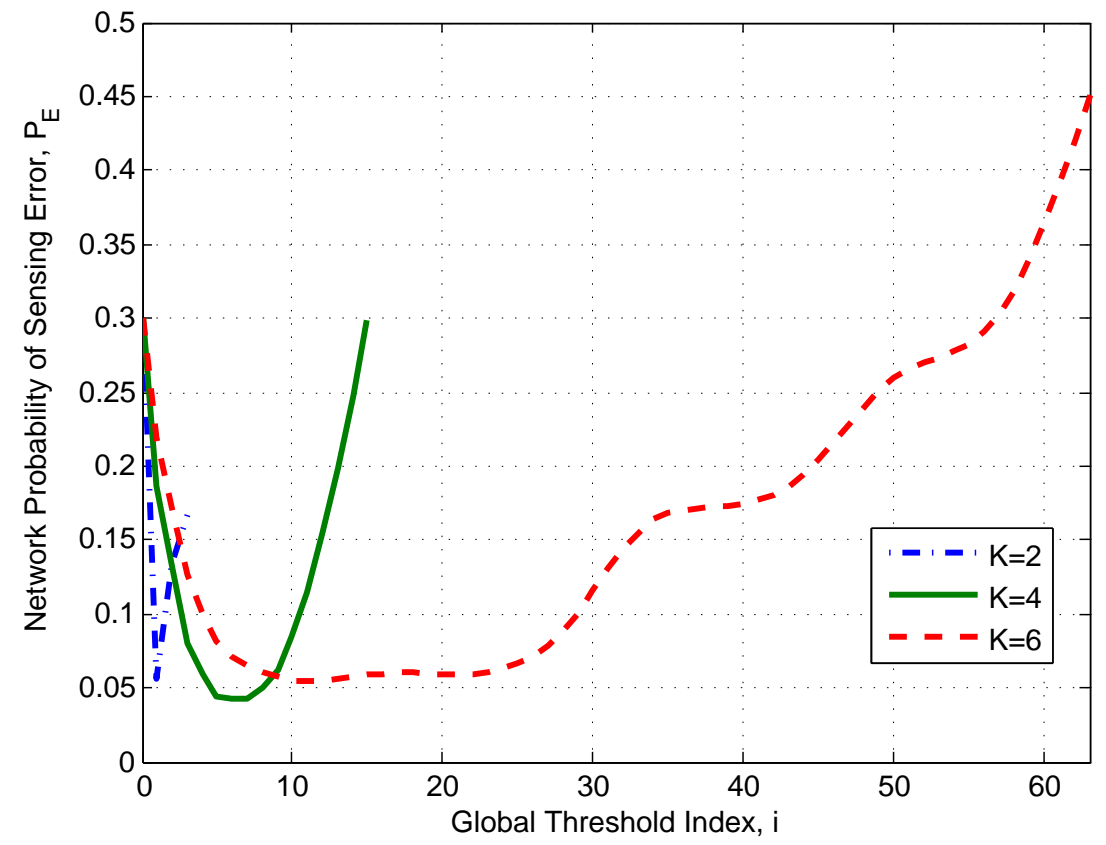

Figure 4.9: Network probability of sensing error versus the global threshold, $i$, for different number of secondary users for $J=25$. 
index, $i$, for different number of secondary users, $K$. The window size is set to be $J=25$. From Figure 4.9, we observe that, as $P_{E}$ varies with the global threshold index $i$, there exists a minimum value for $P_{E}$ that is different for different values $K$. It is clear that, for each value of $K$, using the global threshold, $\lambda^{\text {opt }}$, that corresponds to the optimal global threshold index, $i^{\text {opt }}$, we can obtain a much lower probability of sensing error when compared to using non-optimal settings.

\subsubsection{Dual Search Based Results}

In our dual search algorithm, we use non-linear optimization by mesh adaptive direct search (NOMAD) software package $[113,114]$ to solve the unconstrained minimization problem in (4.25). NOMAD is designed to solve non-linear, non-smooth, noisy optimization problems and can solve non-linear optimization problems with continuous or discrete variables [115].

Table 4.2: Dual Search Algorithm Parameters.

\begin{tabular}{|c|c|}
\hline Parameter & Value \\
\hline Initial multiplier value, $\mu^{0}$ & 5 \\
\hline Convergence tolerance, $\tau$ & $1 \mathrm{e}-5$ \\
\hline Maximum number of iterations & 20 \\
\hline Step size, $\Delta$ & 2 \\
\hline Probability of detection threshold, $\beta$ & 0.9 \\
\hline NOMAD maximum number of iterations & 1500 \\
\hline NOMAD maximum function evaluations & 10000 \\
\hline
\end{tabular}

The results for the dual search algorithm is presented in Table 4.3 with parameters shown in Table 4.2. We see from Table 4.3 that our algorithm can reach the convergence criterion while satisfying the constraint on the minimum probability of detection, $\beta$, for different number of secondary users, $K$, with only few number of iterations. 
Table 4.3: Performance Results of the Dual Search Algorithm.

\begin{tabular}{|l|l|l|l|l|l|}
\hline $\begin{array}{l}\text { Number of } \\
\text { users, } K \\
\text { iterations }\end{array}$ & $\begin{array}{l}\text { Number of } \\
\text { Number of } \\
\text { function } \\
\text { evaluations } \\
\text { (NOMAD) }\end{array}$ & $i^{\text {opt }}$ & $P_{E}\left(\lambda^{\text {opt }}\right)$ & $P_{D}\left(\lambda^{\text {opt }}\right)$ \\
\hline 4 & 6 & 8 & 5 & 0.0394 & 0.9810 \\
\hline 6 & 5 & 8 & 11 & 0.0494 & 0.9956 \\
\hline 8 & 6 & 19 & 77 & 0.0503 & 0.9660 \\
\hline 10 & 5 & 33 & 165 & 0.0503 & 0.9966 \\
\hline 12 & 5 & 38 & 789 & 0.0492 & 0.9921 \\
\hline
\end{tabular}

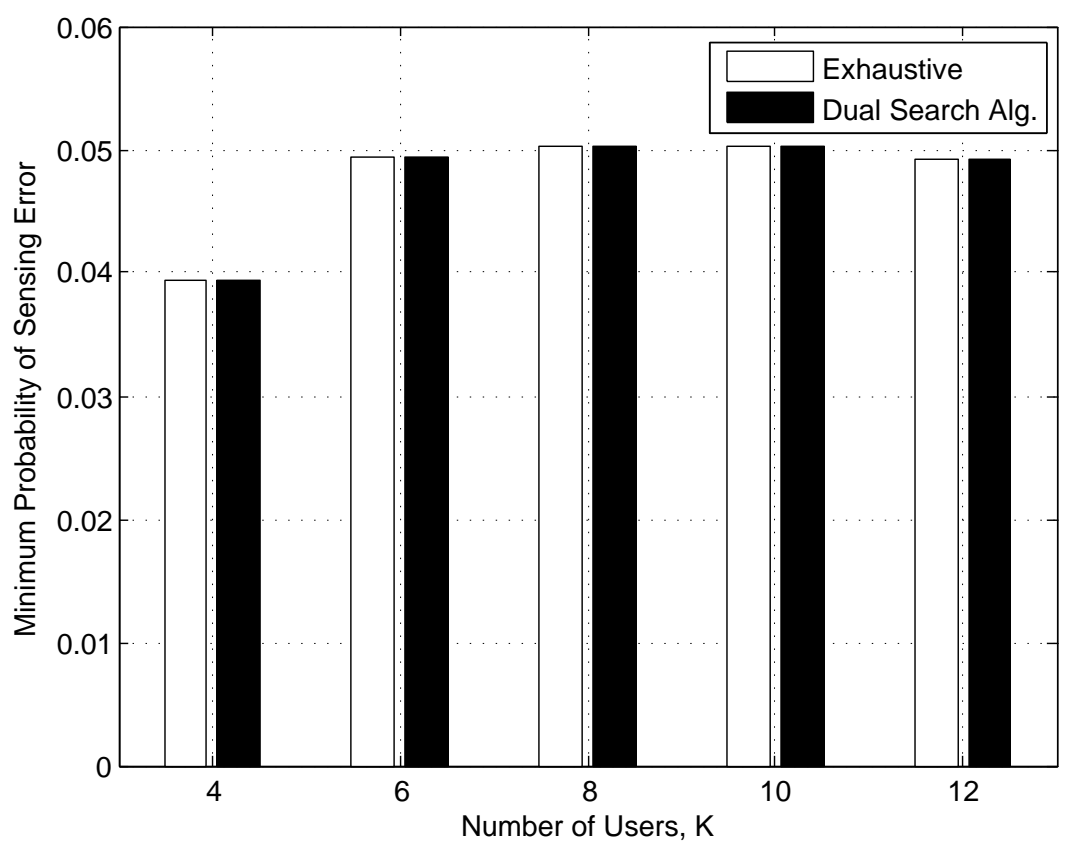

Figure 4.10: Comparision of the minimum probability of sensing error for the dual search algorithm and the exhaustive search for different number of secondary users for $\beta=0.9$.

Next, we evaluate the performance of the dual search algorithm and compare the performance to the exhaustive search for $K \leq 12$. The main drawback of the exhaustive search algorithm is that its complexity increases exponentially with the number of secondary users, $K$. In Figure 4.10, we compare the minimum probability of sensing error obtained from the dual search algorithm and the exhaustive search for different number of secondary users, $K$, for $J=25$ and $\beta=0.9$. We can see from the figure that the dual search algorithm converges 


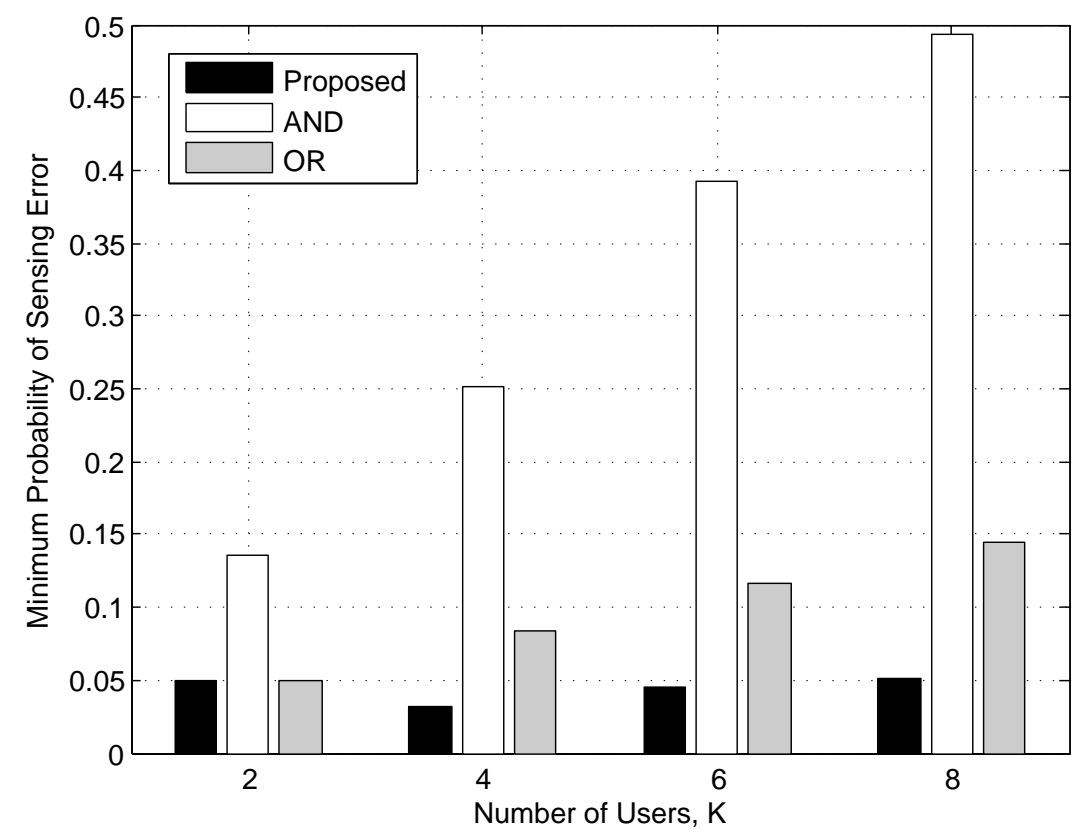

Figure 4.11: Comparision of the minimum probability of sensing error for the proposed scheme and the OR and AND fusion schemes for different number of secondary users for $\beta=0.9$.

to the optimal values of the objective function obtained through the exhaustive search with zero duality gap for different number of secondary users.

In Figure 4.11, we compare the performance of the proposed reliability-based fusion scheme, in terms of the minimum probability of sensing error, to the OR and AND fusion schemes for different number of secondary users. For different values of $K$, with $\beta=0.9$, the minimum probability of error for the proposed scheme is calculated by using the optimum global threshold $\lambda^{\text {opt}}$, which is obtained using the dual search algorithm. From the figure we can see that the proposed scheme can provide lower probability of sensing error when compared to OR and AND fusion rules. As the number of secondary users increases, the performance improvement of the proposed scheme is more pronounced since by allocating different weights to different users, the multiuser diversity gain is better exploited. For example, for $K=8$, the proposed scheme can provide improvement in the network probability of sensing error for up to $18 \%$ and $88 \%$ when compared to the OR and AND fusion rules, 


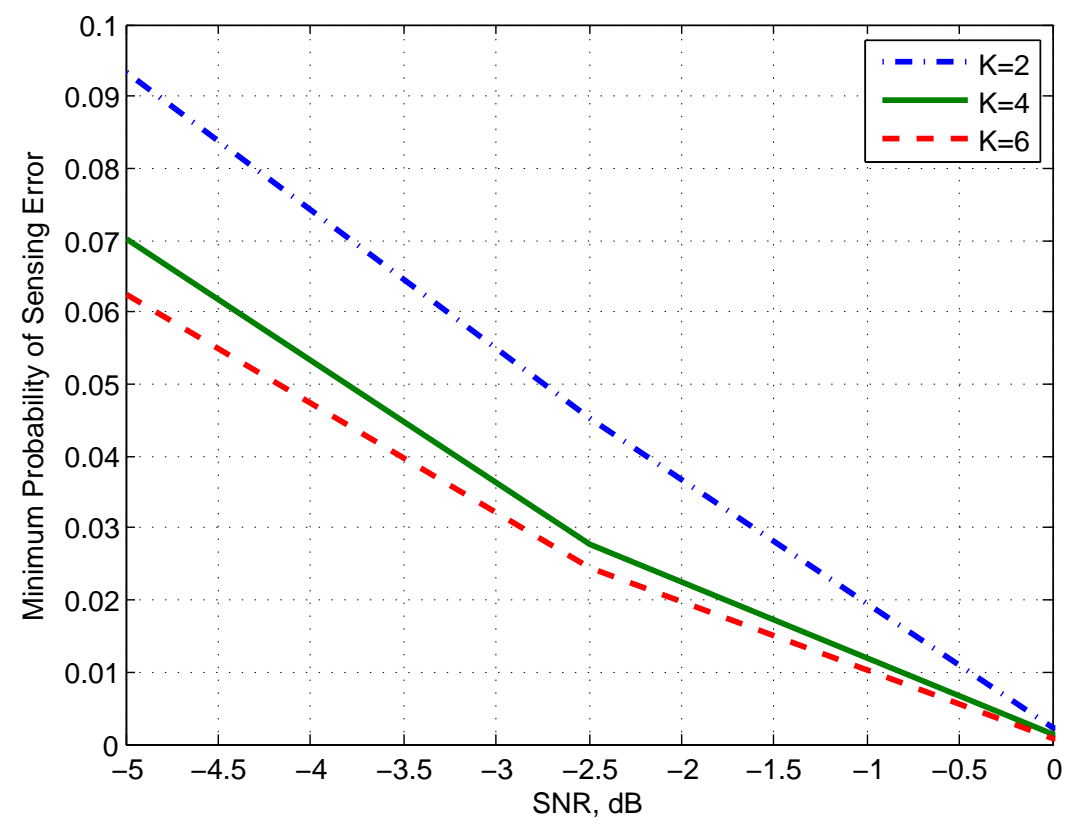

Figure 4.12: Minimum probability of sensing error versus SNR for different number of secondary users for $\beta=0.9$.

respectively.

Figure 4.12 shows the minimum probability of sensing error with $\lambda^{\text {opt }}$, which is obtained using the dual search algorithm, versus SNR for different number of secondary users. It can be seen from the figure that the minimum probability of sensing error decreases as the SNR increases, for all the considered values of $K$. We can also observe from the figure that, as SNR increases, the same minimum probability of sensing can be achieved regardless of the number of users. This is due to the fact that, as SNR increases, each secondary user is able to make a correct sensing decision on its own which makes cooperation less effective.

\subsection{Chapter Summary}

In this chapter, we proposed a reliability-based decision fusion scheme that uses the past global decisions and local secondary decisions to estimate the reliability of the sensing decision made by each secondary user. The reliability of each secondary user is then taken into 
account in the form of a weight factor when making the final decision at the fusion center. We considered heterogeneous secondary users with different probabilities of detection and false alarm that are approximated according to their relative frequencies through a counting process. We formulated the problem of minimizing the network probability of sensing error with heterogeneous secondary users subject to a limit on the network probability of detection as a constrained non-linear integer programming problem. To solve the formulated optimization problem, we employed a non-linear Lagrangian approach, in which the constrained problem is reduced to an unconstrained problem using a non-linear exponential Lagrangian function, and developed a dual search algorithm. We showed through simulation results that the developed dual search algorithm converges to the optimal value, obtained through exhaustive search, with zero duality gap using few number of iterations. We also showed the effect of different parameters on the proposed fusion scheme. For example, our results showed that the window size for which a good estimate of the probabilities of detection and false alarm can be obtained is dependent on the received SNR and the global threshold. Simulation results also showed that the proposed reliability-based scheme is able to provide lower network probability of sensing error when compared to the OR and AND fusion schemes. This improvement is more pronounced as the number of users increases since by assigning weights differently to secondary users, the multiuser diversity gain is better exploited.

In Chapters 3 and 4, we assumed that the local decisions of the secondary users in cooperative spectrum sensing are independent. However, spatially close secondary users are likely to be affected by the same environmental conditions, and therefore, their local decisions are likely to be correlated. On the other hand, secondary users that are farther apart can benefit from their different geographical locations, and obtain more reliable sensing results. Therefore, selecting uncorrelated secondary users for cooperation can improve the robustness of the sensing results with lower number of secondary users which can further help in reducing the cooperation overhead. In the next chapter, we focus on the evaluation of the correlation between the secondary users' local decisions by employing a correlation 
model that is indexed by a single correlation parameter. Using this correlation model, we optimize the number of secondary users, $K$, for the $K$-out-of- $M$ fusion rule that minimizes the network probability of sensing error when the local decisions of the cooperating secondary users are correlated. 


\section{Chapter 5}

\section{Decision Fusion Scheme for CSS with Correlated Local Decisions}

\section{$5.1 \quad$ Introduction}

In cooperative spectrum sensing, correlation between secondary users degrades the sensing performance since it decreases the spatial diversity $[25,36]$. Since spectrum utilization observed by closely located secondary users may be highly correlated due to the inherent spatial correlation in the received primary users' signals and correlated shadow fading, the local decisions of secondary users are also correlated. Although the independence assumption on the local detectors' observations simplifies the performance analysis of cooperative spectrum sensing, this assumption is not practical in the case where the proximity of the secondary users results in correlated observations. To ensure reliable detection, the correlation among different secondary users should also be taken into consideration.

It was shown in [116] that having a small number of secondary users over a large distance may be more effective than a large number of closely located users in correlated shadowing scenarios. Therefore, it is beneficial to select uncorrelated secondary users for cooperation to improve the cooperative gain with fewer number of users. The selection of uncorrelated 
users requires the evaluation of the correlation between secondary users. For this purpose, in this chapter, we employ a correlation model that is indexed by a single parameter, $\rho$, which captures the correlation characteristics of the secondary users' local decisions. We then analyze the impact of correlated secondary users' local decisions on the performance of cooperative spectrum sensing when the $K$-out-of- $M$ fusion rule is employed at the fusion center. We derive the network probabilities of detection and false alarm when the secondary users' local decisions are correlated under both hypothesis. Our performance evaluations are based on two performance criteria which are the Neyman Pearson and the minimization of the probability of sensing error criteria. We formulate the problem of minimizing the network probability of sensing error under the correlation model used in our analysis as a mixed integer non-linear programming (MINLP) problem which is generally NP-hard [91]. To solve the formulated optimization problem, we employ the genetic algorithm to find the optimal assignments for the number of secondary users, $K$, and the local sensing threshold for a certain correlation index. The genetic algorithm is a popular meta-heuristic that is particularly well suited for multi-objective problems as the one considered in this chapter $[117]$.

\subsection{Related Work}

For the case of independent secondary users' observations, it was shown in [49] that cooperating with all users in the network does not necessarily achieve the optimum performance. The authors in [49] used constant detection rate and constant false alarm rate for optimally selecting the secondary users with highest primary user's signal-to-noise ratio (SNR) for cooperation using AND and OR fusion rules. In [118], it was shown that the optimal fusion rule when the sensor observations are independent is a majority voting (MV) rule in the case of binary local detectors. In [119], the authors considered optimizing the $K$ value of the $K$-out-of- $M$ fusion rule and found that the optimal decision fusion rule to minimize the total 
probability of sensing error can be approximated as a majority voting rule. In [120], it was shown that cooperative sensing is most effective when secondary users observe independent fading or shadowing.

Under log-normal shadowing, the observations of two closely located secondary users may be correlated due to their proximity. In this case, secondary users experience similar shadowing effects called spatially correlated shadowing. In [36], it was shown that spatial correlation due to shadowing can degrade the detection performance and compromise the achievable cooperative gain. Performance evaluation of cooperative spectrum sensing under correlated log-normal shadowing in both sensing and reporting channels was investigated in [121]. A correlation model $[36,122]$ derived from empirical data with decaying exponential function is commonly used to determine the spatial correlation in urban and suburban environments. In general, the spatial correlation between two secondary users exponentially decreases as the distance between these two increases, and secondary users can be considered uncorrelated if the distance is larger than the so called de-correlation distance. In [123], the performance degradation of cooperative sensing under exponentially-correlated log-normal shadowing was investigated in terms of missing opportunities. In [17], the problem of fusing the decisions made at the local detectors when the secondary users observe conditionally dependent data due to correlated shadowing was studied and a suboptimal temporal detector was proposed based on a linear quadratic (LQ) detector, which uses partial statistical knowledge to improve detection performance. The results in [17] showed that the suboptimal LQ detector outperforms the counting rule only when the correlation between the secondary users is strong. Also, their results were based on the assumption that the noise observations are independent. This assumption might not hold if the physical proximity of the local detectors results in the noise on each detector being dependent [124].

In [55], a correlation-aware user selection algorithm was developed to address the dynamic changes in the spatial correlation experienced by mobile secondary users. To accurately derive the spatial correlation coefficient, a correlation model between mobile secondary users 
was first developed. Based on this correlation model, a distributed user selection algorithm that adaptively selects uncorrelated secondary users through the spatial correlation coefficient was designed. In [125], a joint spatial-temporal sensing scheme for CR networks was proposed, where secondary users collaboratively estimate the location and transmit power of the primary transmitter. Based on those estimates, secondary users determine their maximum allowable transmission power, and use the location information to decide which users should participate in cooperative sensing in order to minimize the correlation among the secondary users' observations.

The performance of a distributed detection system with given local decision rules and correlated local decisions was studied in [126] and the optimum decision fusion rule in the Neyman-Pearson sense was derived and analyzed. In [124], the probability of detection was evaluated using the Neyman-Pearson criterion when the fusion rule was fixed to one of the standard rules such as AND, OR or MV rule. The authors considered equi-correlated observations with identical detectors. The authors also studied the asymptotic performance of distributed and centralized detection systems in the presence of correlated Gaussian noise in [127]. They considered two correlation models, one where the correlation between observations from any two sensors decreases geometrically as their separation increases and the other where the correlation between observations from each pair of sensors is identical. The optimal fusion rule was developed for correlated local binary decisions, in terms of the conditional correlation coefficients of all orders in [128]. In [129], the authors considered extending the classical locally optimum detection results to the case of distributed detection with dependent sensor observations. The study in [129] focused on the case of known signals in correlated Gaussian noise and the authors gave simple conditions under which likelihood ratio tests are optimum at the sensors when the Bayesian criterion was considered. In [130], the authors proposed an adaptive fusion algorithm, which requires only the knowledge of few system parameters, for an environment where the observations and local decisions are dependent from one sensor to another. 
In this thesis work, we study the performance of cooperative spectrum sensing when correlated local decisions are fused at the fusion center with known correlation index, $\rho$. The relationship between the correlation coefficient between two sensors' local decisions and the correlation coefficient between those sensors' local observations was studied in [127]. This study showed that the correlation coefficient between two sensors' decisions cannot exceed the correlation coefficient between the corresponding sensors' observations. In the next section, we present the correlation model used to analyze the performance of cooperative spectrum sensing with correlated local decisions.

\subsection{Correlation Model}

In this chapter, we consider a cognitive radio network with $M$ secondary users that employ the energy detection technique presented in section 3.3.1. We consider the same system model described in section 3.3, with the same assumption on the noise and primary user's signal. That is, the noise, $v_{k}(n)$, is a real Gaussian noise with zero mean and variance $\sigma_{v}^{2}$ and the primary user's signal, $s(n)$, is an independent identically distributed (i.i.d.) random process with mean zero and variance $\sigma_{s}^{2}$ and is BPSK modulated.

We consider the case in which each individual secondary user, $k, k=1 \cdots M$, makes a one-bit hard decision, $d_{k}$, on the absence or presence of the primary user's transmission based on the sensing information, such that,

$$
d_{k}= \begin{cases}1, & \text { if } Y_{k}>\gamma_{k} \\ 0, & \text { if } Y_{k} \leq \gamma_{k}\end{cases}
$$

Each secondary user then sends this one-bit decision to the fusion center which makes the final decision regarding the occupancy of each channel. We further assume that the local 
decisions are correlated and the correlation coefficients are given by

$$
\begin{aligned}
E\left[\prod_{k \in I^{\prime}} d_{k} \mid H_{x}\right] & =E\left[d_{k_{1}} d_{k_{2}} \cdots d_{k_{v}} \mid H_{x}\right] \\
& =P\left(d_{k_{1}}=1, d_{k_{2}}=1, \cdots, d_{k_{v}}=1 \mid H_{x}\right)
\end{aligned}
$$

where $E\left[u \mid H_{x}\right]$ and $P\left[u \mid H_{x}\right]$ are the conditional expectation and conditional probability given $H_{x}$, where $x \in\{0,1\}$, respectively, $I^{\prime} \subseteq\{1,2,3, \ldots, M\},\left|I^{\prime}\right|=m$ is the cardinality number of set $I^{\prime}$ and $k_{v} \in I^{\prime}, v=1, \ldots, m$.

By definition [126],

$$
E\left[\prod_{k \in I^{\prime}} d_{k} \mid H_{x}\right]=1, \quad \text { if } \quad I^{\prime}=\Phi
$$

where $\Phi$ is the empty set.

For $\left|I^{\prime}\right|=1$, we have,

$$
E\left[d_{k} \mid H_{1}\right]=P\left(d_{k}=1 \mid H_{1}\right)=P_{d, k}
$$

and

$$
E\left[d_{k} \mid H_{0}\right]=P\left(d_{k}=1 \mid H_{0}\right)=P_{f, k}
$$

where $P_{d, k}$ and $P_{f, k}$ are the local probabilities of detection and false alarm of the $k^{t h}$ secondary user, respectively.

The correlation coefficient, $\rho_{y_{1} y_{2}}^{x}$, between two local decisions $d_{y_{1}}$ and $d_{y_{2}}$ under hypothesis $H_{x}$ is given by,

$$
\rho_{y_{1} y_{2}}^{x}=\frac{E\left[d_{y_{1}} d_{y_{2}} \mid H_{x}\right]-E\left[d_{y_{1}} \mid H_{x}\right] E\left[d_{y_{2}} \mid H_{x}\right]}{\sqrt{\left(E\left[d_{y_{1}}^{2} \mid H_{x}\right]-\left(E\left[d_{y_{1}} \mid H_{x}\right]\right)^{2}\right)\left(E\left[d_{y_{2}}^{2} \mid H_{x}\right]-\left(E\left[d_{y_{2}} \mid H_{x}\right]\right)^{2}\right)}}
$$

where $x \in\{0,1\}, y_{1}$ and $y_{2} \in\{1 \cdots M\}$ and $y_{1} \neq y_{2}$. Since $d_{y_{1}} \in\{0,1\}$, it follows that $d_{y_{1}}=\left(d_{y_{1}}\right)^{2}$. Therefore,

$$
\rho_{y_{1} y_{2}}^{x}=\frac{E\left[d_{y_{1}} d_{y_{2}} \mid H_{x}\right]-E\left[d_{y_{1}} \mid H_{x}\right] E\left[d_{y_{2}} \mid H_{x}\right]}{\sqrt{E\left[d_{y_{1}} \mid H_{x}\right] E\left[d_{y_{2}} \mid H_{x}\right]\left(1-E\left[d_{y_{1}} \mid H_{x}\right]\right)\left(1-E\left[d_{y_{2}} \mid H_{x}\right]\right)}} .
$$


Assuming the distance between any two secondary users is small compared to the distance between the secondary users and the primary transmitter, the received signal at each secondary user will experience almost identical path loss $[76,119,131]$. Therefore, in the case of an AWGN environment, we can assume equal SNR for the different secondary users [119]. Assuming the same threshold for all secondary users, $\gamma_{k}=\gamma$, we have $P_{f, k}=P_{f}$. In the case of an AWGN channel as previously assumed, we will also have $P_{d, k}=P_{d}$. Since $E\left[d_{y_{1}} \mid H_{x}\right]=E\left[d_{y_{2}} \mid H_{x}\right]$ from (5.4) and (5.5), $\rho_{y_{1} y_{2}}^{x}$ is independent of $y_{1}$ and $y_{2}$ and every pair of local detectors is equally correlated [132]. Therefore,

$$
\rho^{1}=\frac{P\left[d_{y_{1}}=1, d_{y_{2}}=1 \mid H_{1}\right]-P_{d}^{2}}{P_{d}\left(1-P_{d}\right)},
$$

and

$$
\rho^{0}=\frac{P\left[d_{y_{1}}=1, d_{y_{2}}=1 \mid H_{0}\right]-P_{f}^{2}}{P_{f}\left(1-P_{f}\right)},
$$

where $\rho^{1}$ and $\rho^{0}$ are the correlation coefficients under $H_{1}$ and $H_{0}$, respectively. It is clear from (5.8) and (5.9) that $\rho^{x}$ varies only with $P\left[d_{y_{1}}=1, d_{y_{2}}=1 \mid H_{x}\right]$ which is a function of only $P_{d}$ under $H_{1}$ and a function of only $P_{f}$ under $H_{0}$ [133].

If we assume that the fusion center has the input vector $D=\left[d_{1}, d_{2}, \cdots, d_{M}\right]$, where $D$ can take $2^{M}$ possible realizations, the joint probability $P\left(D \mid H_{x}\right)$ at the fusion center will be given by $[126]$ :

$$
P\left(D \mid H_{x}\right)=\sum_{k=0}^{r}(-1)^{k}\left(\begin{array}{l}
r \\
k
\end{array}\right) \epsilon_{M-r+k}^{x} .
$$

If $A_{\mu}^{\prime}=\left\{k: d_{k}=\mu\right\}$, such that, $1<k<M$ and $\mu=0,1$, then $r=\left|A_{0}^{\prime}\right|$ and $M-r=\left|A_{1}^{\prime}\right|$ denote the number of secondary users that decide in favor of $H_{0}$ and $H_{1}$, respectively, and,

$$
\begin{aligned}
\epsilon_{y}^{x} & =E\left[d_{k_{1}} d_{k_{2}} \cdots d_{k_{y}} \mid H_{x}\right] \\
& =\epsilon_{1}^{x} \prod_{s=0}^{y-2} \frac{\rho^{x}\left(s+1-\epsilon_{1}^{x}\right)+\epsilon_{1}^{x}}{1+s \rho^{x}} \quad \text { for } \quad y \geq 2,
\end{aligned}
$$

where $y$ is the number of secondary detectors sending their decision to the fusion center, 
$\rho^{x}$ is the correlation index under $H_{x}, x \in\{0,1\}$. For $y<2, \epsilon_{1}^{0} \triangleq E\left[d_{y_{1}} \mid H_{0}\right]=P_{f}$ and $\epsilon_{1}^{1} \triangleq E\left[d_{y_{1}} \mid H_{1}\right]=P_{d}$ and by definition, $\epsilon_{0}^{1}=\epsilon_{0}^{0}=1$.

\subsection{Decision Fusion with Correlated Local Decisions}

In this chapter, we consider the general $K$-out-of- $M$ fusion rule as the decision fusion rule employed at the fusion center, where $K$ is the number of secondary users that claim that the primary user is present and $M$ is the total number of cooperating users with correlated local decisions. We derive the network probability of detection, $P_{D}$, and network probability of false alarm, $P_{F}$, for this fusion rule when the secondary users' local decisions are correlated. For the sake of comparison, we give special attention to some special cases of the $K$-out-of- $M$ fusion rule which are the majority voting, OR and AND fusion rules.

\subsubsection{General $K$-out-of- $M$ Fusion Rule}

In the $K$-out-of- $M$ fusion rule, if $K$ users or more decide in favor of $H_{1}$, i.e., if $M-r \geq$ $K$, where $r$ is as defined in (5.10), then the final decision declares that a primary user's transmission is present. We define an indicator function for the $K$-out-of- $M$ system as,

$$
\varphi(D)= \begin{cases}1, & \text { if } \sum_{k=1}^{M} d_{k} \geq K \\ 0, & \text { if } \sum_{k=1}^{M} d_{k}<K\end{cases}
$$

Therefore, we can calculate the network probability of detection for the $K$-out-of- $M$ fusion rule from the joint probability of the local decisions, defined in (5.10), when the input vector $D$ has at least $K$ decisions equal to one. Therefore,

$$
P_{D}=P\left(\varphi(D)=1 \mid H_{1}\right)=\sum_{z=0}^{M-K} \sum_{k=0}^{M-K-z}(-1)^{k}\left(\begin{array}{c}
M \\
K+z
\end{array}\right)\left(\begin{array}{c}
M-K-z \\
k
\end{array}\right) \epsilon_{K+z+k}^{1},
$$

where $\epsilon_{y}^{x}$ is defined in (5.11). 
Similarly, we derive the network probability of false alarm, $P_{F}$, as

$$
P_{F}=P\left(\varphi(D)=1 \mid H_{0}\right)=\sum_{z=0}^{M-K} \sum_{k=0}^{M-K-z}(-1)^{k}\left(\begin{array}{c}
M \\
K+z
\end{array}\right)\left(\begin{array}{c}
M-K-z \\
k
\end{array}\right) \epsilon_{K+z+k}^{0}
$$

\subsubsection{Majority Voting (MV) Fusion Rule}

In the majority voting rule, the final decision is based on majority of the individual decisions, i.e., $K=\left\lceil\frac{M}{2}\right\rceil$ in $(5.13)$ and (5.14), where $\lceil x\rceil$ denotes the smallest integer greater than $x$. Therefore, we can express $P_{D}$ as

$$
P_{D}=\sum_{z=0}^{M-\left\lceil\frac{M}{2}\right\rceil} \sum_{k=0}^{M-\left\lceil\frac{M}{2}\right\rceil-z}(-1)^{k}\left(\begin{array}{c}
M \\
\left\lceil\frac{M}{2}\right\rceil+z
\end{array}\right)\left(\begin{array}{c}
M-\left\lceil\frac{M}{2}\right\rceil-z \\
k
\end{array}\right) \epsilon_{\left\lceil\frac{M}{2}\right\rceil+z+k}^{1},
$$

and we can express $P_{F}$ as

$$
P_{F}=\sum_{z=0}^{M-\left\lceil\frac{M}{2}\right\rceil} \sum_{k=0}^{M-\left\lceil\frac{M}{2}\right\rceil-z}(-1)^{k}\left(\begin{array}{c}
M \\
\left\lceil\frac{M}{2}\right\rceil+z
\end{array}\right)\left(\begin{array}{c}
M-\left\lceil\frac{M}{2}\right\rceil-z \\
k
\end{array}\right) \epsilon_{\left\lceil\frac{M}{2}\right\rceil+z+k}^{0} .
$$

\subsubsection{OR Fusion Rule}

In the OR fusion rule, the fusion center decides that the primary user is absent only if all secondary users decide the absence of the primary user's signal [50], i.e., setting $K=1$ in (5.13) and (5.14). This is equivalent to the following simplified form:

$$
\begin{aligned}
P_{D} & =1-P\left(d_{1}=0, d_{2}=0, \cdots, d_{M}=0 \mid H_{1}\right) \\
& =1-\sum_{k=0}^{M}(-1)^{k}\left(\begin{array}{c}
M \\
k
\end{array}\right) \epsilon_{k}^{1},
\end{aligned}
$$


and

$$
\begin{aligned}
P_{F} & =1-P\left(d_{1}=0, d_{2}=0, \cdots, d_{M}=0 \mid H_{0}\right) \\
& =1-\sum_{k=0}^{M}(-1)^{k}\left(\begin{array}{c}
M \\
k
\end{array}\right) \epsilon_{k}^{0} .
\end{aligned}
$$

\subsubsection{AND Fusion Rule}

In the AND fusion rule, if all local detectors decide that there is a primary user, then the final decision at the fusion center declares that there is a primary user, i.e., setting $K=M$ in (5.13) and (5.14). This is equivalent to the following simplified form:

$$
P_{D}=P\left(d_{1}=1, d_{2}=1, \cdots, d_{M}=1 \mid H_{1,}\right)=\epsilon_{M}^{1},
$$

and

$$
P_{F}=P\left(d_{1}=1, d_{2}=1, \cdots, d_{M}=1 \mid H_{0}\right)=\epsilon_{M}^{0} .
$$

\subsection{CSS Based on Neyman-Pearson Criterion}

We first look at the receiver operating characteristic (ROC), which shows the relationship between the network probability of detection, $P_{D}$, and the network probability of false alarm, $P_{F}$, for three fusion rules which are the majority voting rule, OR and AND fusion rules. The mechanism of obtaining the ROC can be summarized as follows:

- We set the probability of false alarm at the fusion center, $P_{F}$, to a desired target value and obtain the individual secondary users' probability of false alarm, $P_{f, k}$, which is considered equal for all users and has a value of $P_{f}$, numerically from $(5.16),(5.18)$ and (5.20) for the majority voting rule, OR and AND fusion rules, respectively.

- After computing $P_{f}$, where $P_{f}=\epsilon_{1}^{0}$, the individual probability of detection for each secondary user can be obtained by substituting (3.7) into (3.6) to eliminate the thresh- 
old, $\gamma=\gamma_{N P}$, where $\gamma_{N P}$ is the threshold defined for the Neyman-Pearson criterion. Therefore,

$$
P_{d, k}=Q\left(\frac{1}{\sqrt{\left(\frac{2\left|h_{k}\right|^{2} \sigma_{s}^{2}}{\sigma_{v}^{2}}+1\right)}}\left(Q^{-1}\left(P_{f}\right)-\sqrt{\frac{N}{2}} \frac{\left|h_{k}\right|^{2} \sigma_{s}^{2}}{\sigma_{v}^{2}}\right)\right) .
$$

- The network probability of detection, $P_{D}$, for the majority voting rule, OR and AND fusion rules can then be obtained by substituting $P_{d, k}=P_{d}=\epsilon_{1}^{1}$ in (5.15), (5.17) and (5.19), respectively.

We plot the receiver operating characteristic (ROC) curves for the different fusion rules when the local decisions of the secondary users are correlated. The degree of correlation between the secondary local decisions is described by the parameter $\rho$ which is assumed equal under the two hypothesis $H_{0}$ and $H_{1}[130,132]$. We consider a network with $M$ secondary users participating in the decision fusion, and fix the fusion rule to one of three aforementioned rules. The number of detection symbols, $N$, is set to 100 and we consider cooperating secondary users that have the same received SNR [119].

In Figure 5.1, we plot the ROC curves for MV rule for different values of the correlation index, $\rho$, when $M=5$ and $\mathrm{SNR}$ is set to $-10 \mathrm{~dB}$. From the figure we observe that, for a given $P_{F}$, the highest $P_{D}$ is obtained when $\rho=0$, which represents the case when secondary observations are independent. The value of $P_{D}$ then decreases with the increase in the correlation between the observations of the secondary users until eventually the system is reduced to the case of single secondary user (no cooperation) as $\rho$ becomes closer to 1 . Our results show that, for low values of $P_{F}\left(P_{F}<0.1\right)$, depending on the fusion rule considered, we can have up to $7 \%$ to $13 \%$ increase in the system probability of detection when $\rho$ decreases from 0.6 to 0.1 .

Figures 5.2 and 5.3 show the ROC curves for different number of cooperating users $K$ with $\rho=0.05$ and 0.2 , respectively when $M=5$ and the SNR is set to $-10 \mathrm{~dB}$. From Figure 


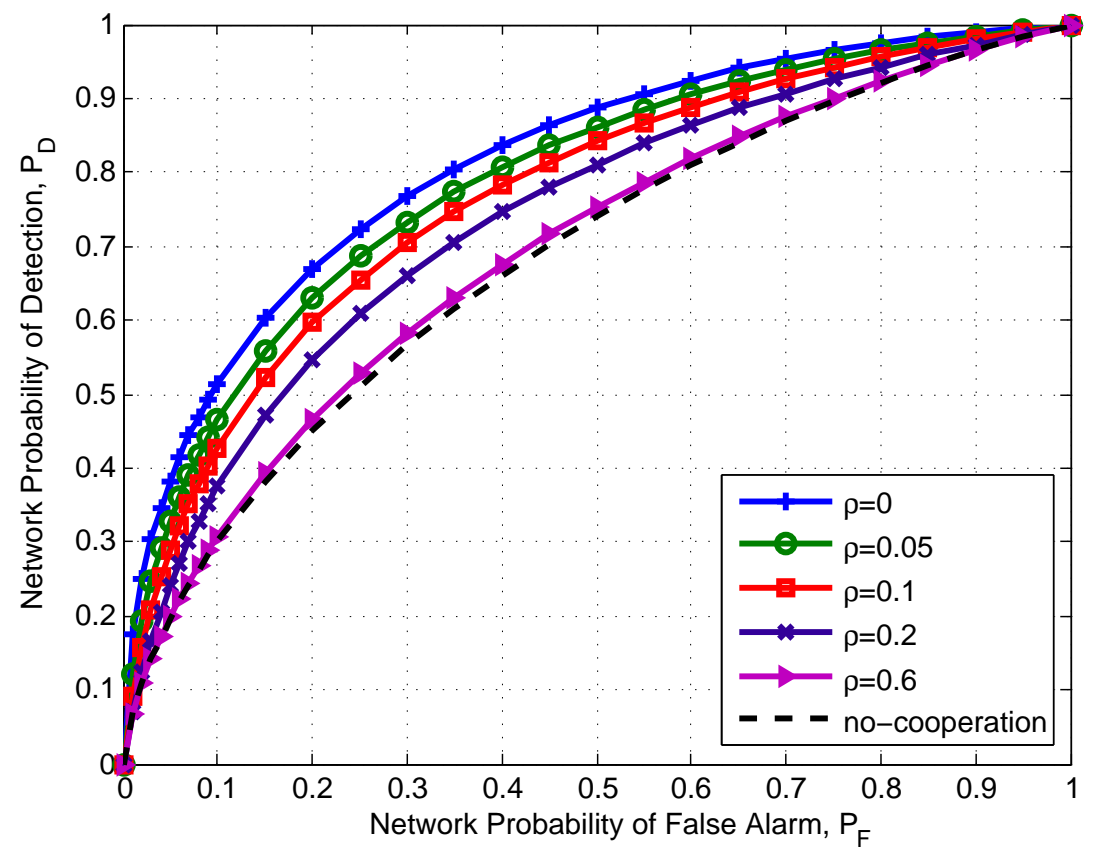

Figure 5.1: ROC curves $\left(P_{D}\right.$ vs. $\left.P_{F}\right)$ for different correlation index for majority voting fusion rule with $M=5$ and SNR $=-10 \mathrm{~dB}$.

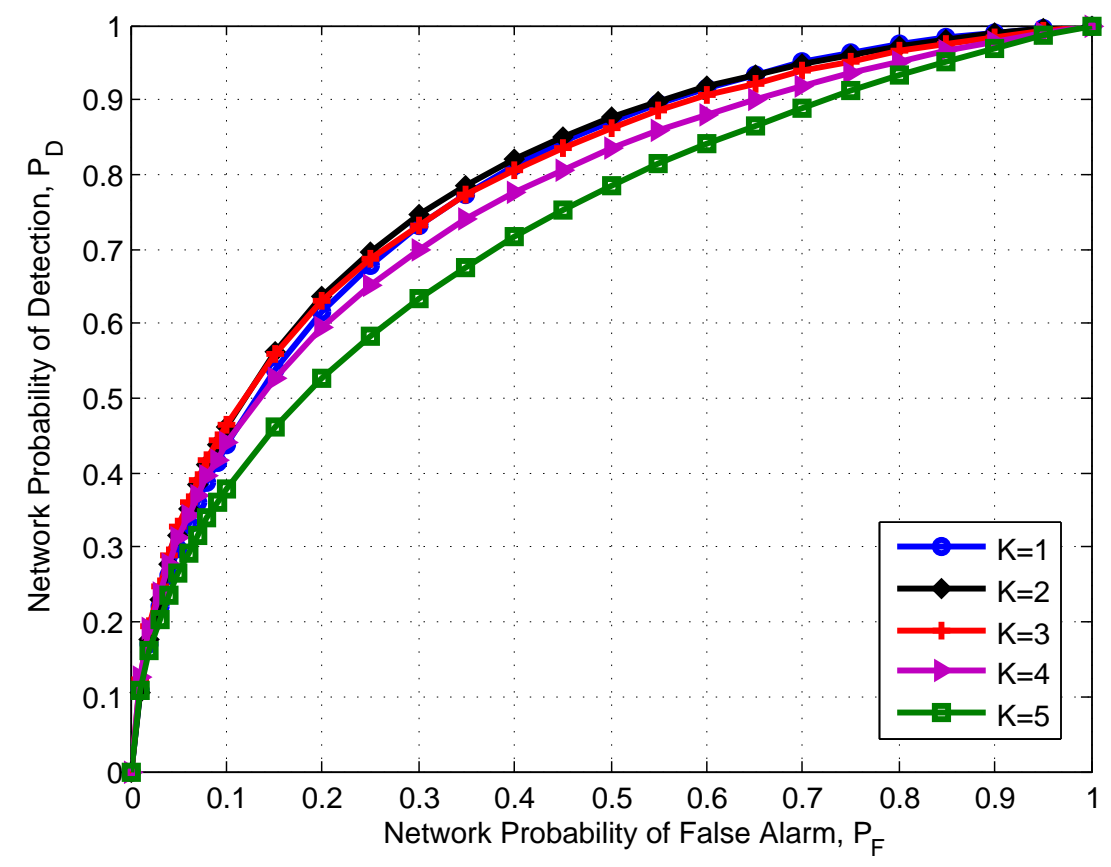

Figure 5.2: Comparison of ROC curves $\left(P_{D}\right.$ vs. $\left.P_{F}\right)$ for different number of secondary users, $K$, when $\rho=0.05$. 


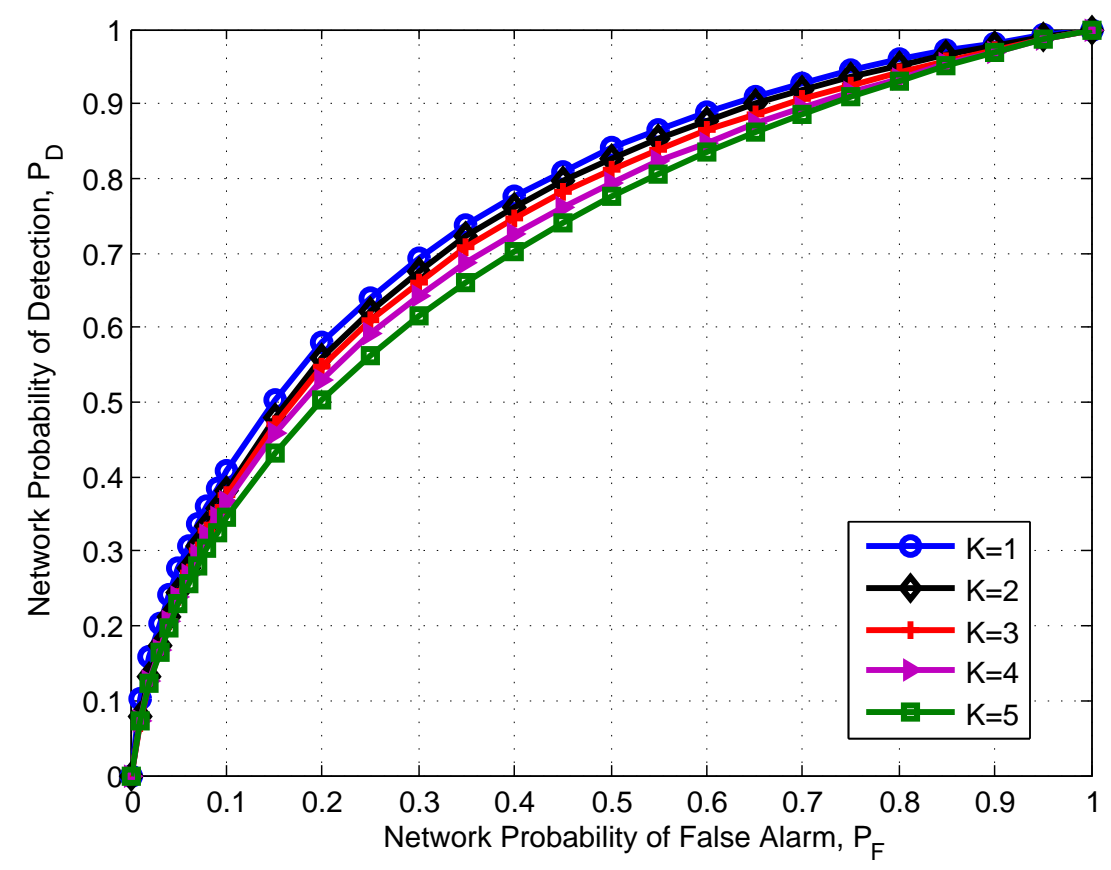

Figure 5.3: Comparison of ROC curves $\left(P_{D}\right.$ vs. $\left.P_{F}\right)$ for different number of secondary users, $K$, when $\rho=0.2$.

5.2 , we notice that, when $\rho=0.05$, although the MV rule $(K=3)$ is not the optimal fusion rule, the MV fusion rule still outperforms the OR $(K=1)$ and $\operatorname{AND}(K=5)$ fusion rules, in terms of the probability of detection, for low values of the network probability of false alarm $\left(P_{F}<0.1\right.$ for the considered case). As $P_{F}$ increases, the OR and AND fusion rules get progressively closer to the MV rule. From Figure 5.3, we observe that, as the correlation index, $\rho$, increases (from our simulation when $\rho>0.1$ ), the OR fusion rule outperforms all the considered fusion rules.

Next, we evaluate the performance of cooperative spectrum sensing with correlated local decision under the Neyman-Pearson criterion. In Figures 5.4 and 5.5, we plot the network probability of detection, $P_{D}$, versus the correlation index, $\rho$, for different number of cooperating users. We compare the performance of the OR, AND and majority voting rules for $M=5$ and $M=10$, respectively, when $P_{F}$ is fixed to 0.1 . From the figures, we observe that, for the three fusion rules and with different number of cooperating users, the highest $P_{D}$ is obtained when the secondary local decisions are independent $(\rho=0)$. The probability 


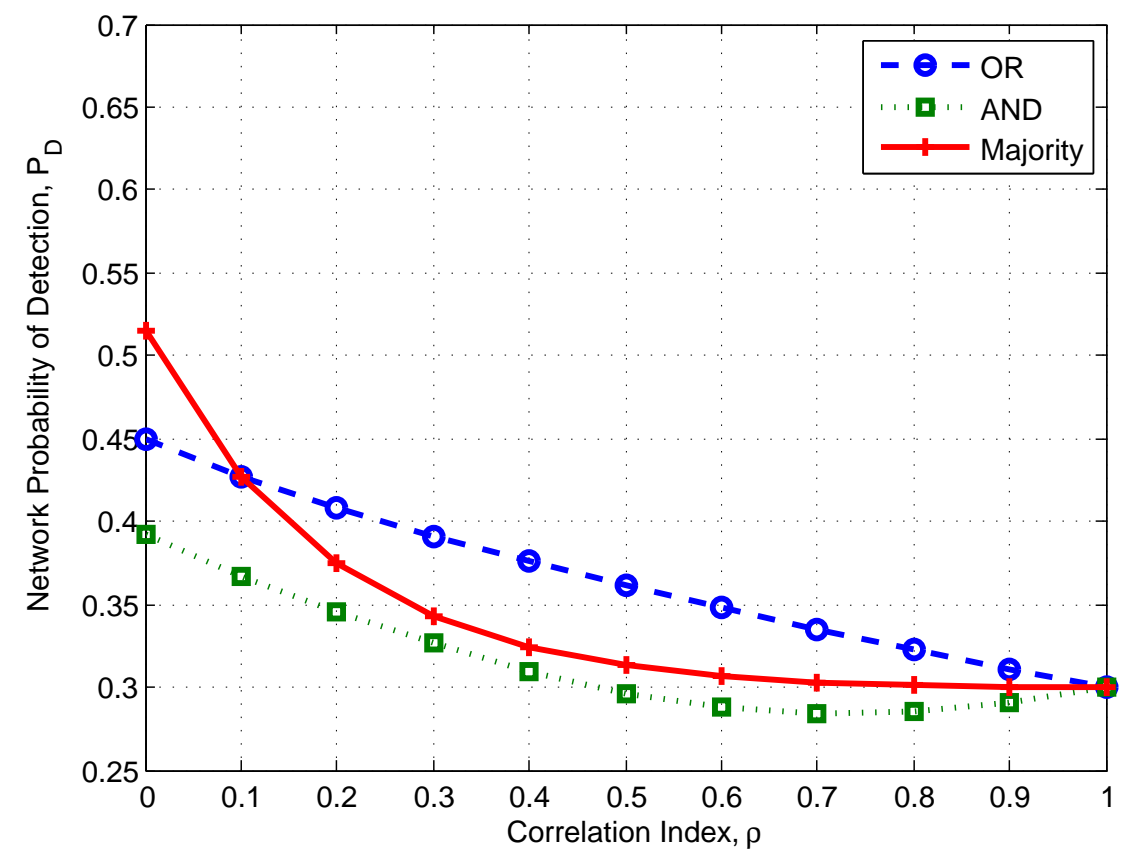

Figure 5.4: Network probability of detection versus correlation index for OR, AND and majority voting rules when $M=5$ for $P_{F}=0.1$ and $\mathrm{SNR}=-10 \mathrm{~dB}$.

$P_{D}$ then decreases with the increase in the correlation index until eventually no significant performance gain can be obtained from cooperation as $\rho$ becomes closer 1 . We also notice that, for all values of $\rho$ less than 1 , increasing the number of cooperating secondary users increases the network probability of detection due to multiuser diversity. We can also see from the figures that the value of the correlation index, $\rho$, below which the majority voting rule is superior depends on the number of cooperating users for a certain SNR and $P_{F}$.

Figure 5.6 shows the network probability of detection versus the SNR when $\rho=0$ and 0.6 with $M=5$ and $P_{F}=0.1$. We observe that, when $\rho=0$, the majority voting rule outperforms the OR and AND rule for low values of the SNR. As the value of $\rho$ increases, the OR fusion rule gives better performance compared to the other two rules for low values of the SNR. From the figure, we also notice that, as $\rho$ increases, for the same network probability of false alarm, higher SNR is required to achieve the same network probability of detection for all the fusion rules considered. For example, if $P_{D}$ is fixed at 0.9 , approximately $3 \mathrm{~dB}$ is required to compensate for the correlation effect (when $\rho$ increases from 0 to 0.6 ) for 


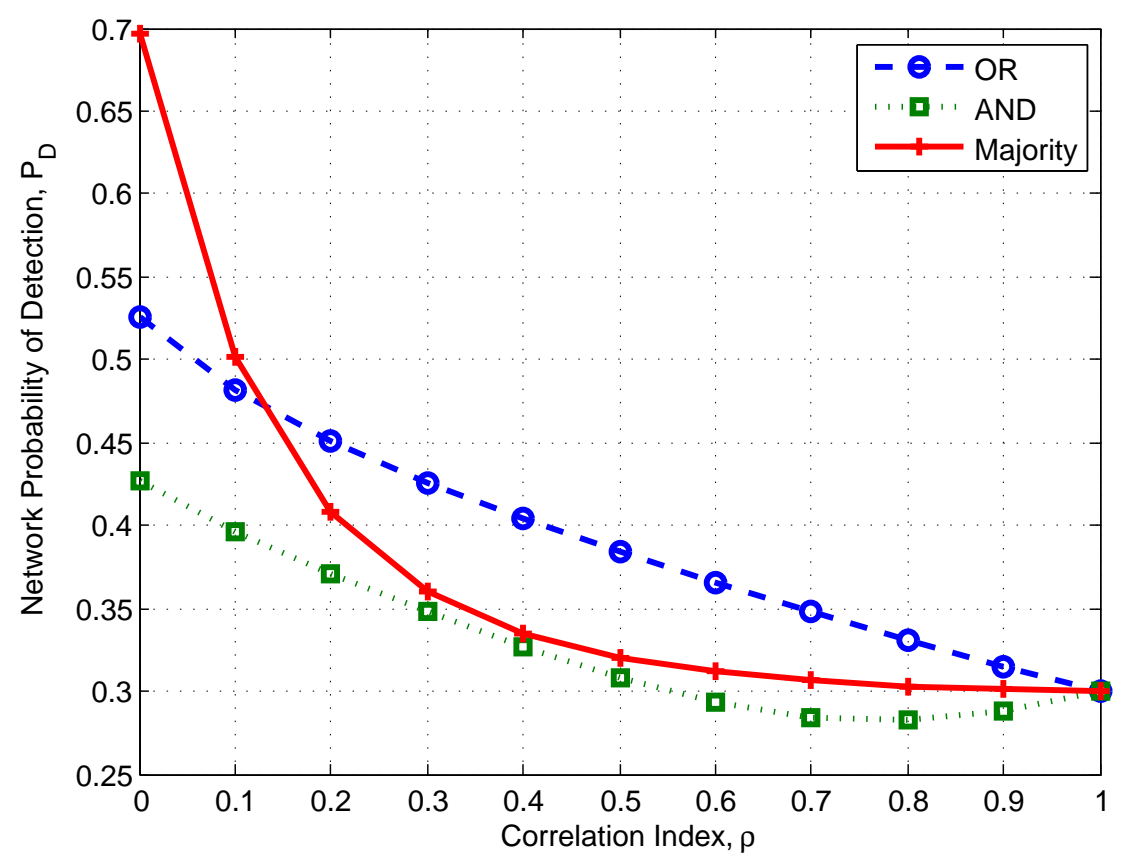

Figure 5.5: Network probability of detection versus correlation index for OR, AND and majority voting rules when $M=10$ for $P_{F}=0.1$ and $\mathrm{SNR}=-10 \mathrm{~dB}$.

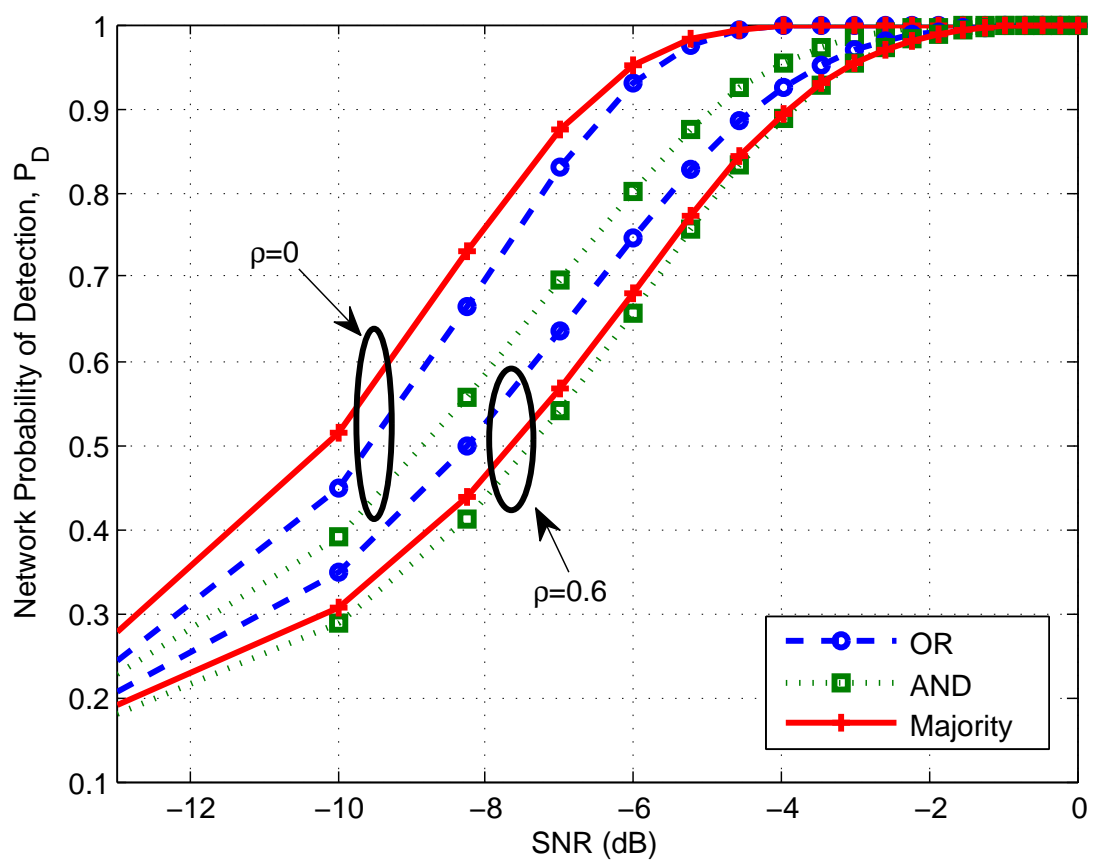

Figure 5.6: Network probability of detection versus SNR for different values of $\rho$ when $P_{F}=0.1$ and $M=5$. 
the majority voting rule and approximately $2 \mathrm{~dB}$ and $1 \mathrm{~dB}$ are required for the $\mathrm{OR}$ and $\mathrm{AND}$ fusion rules, respectively.

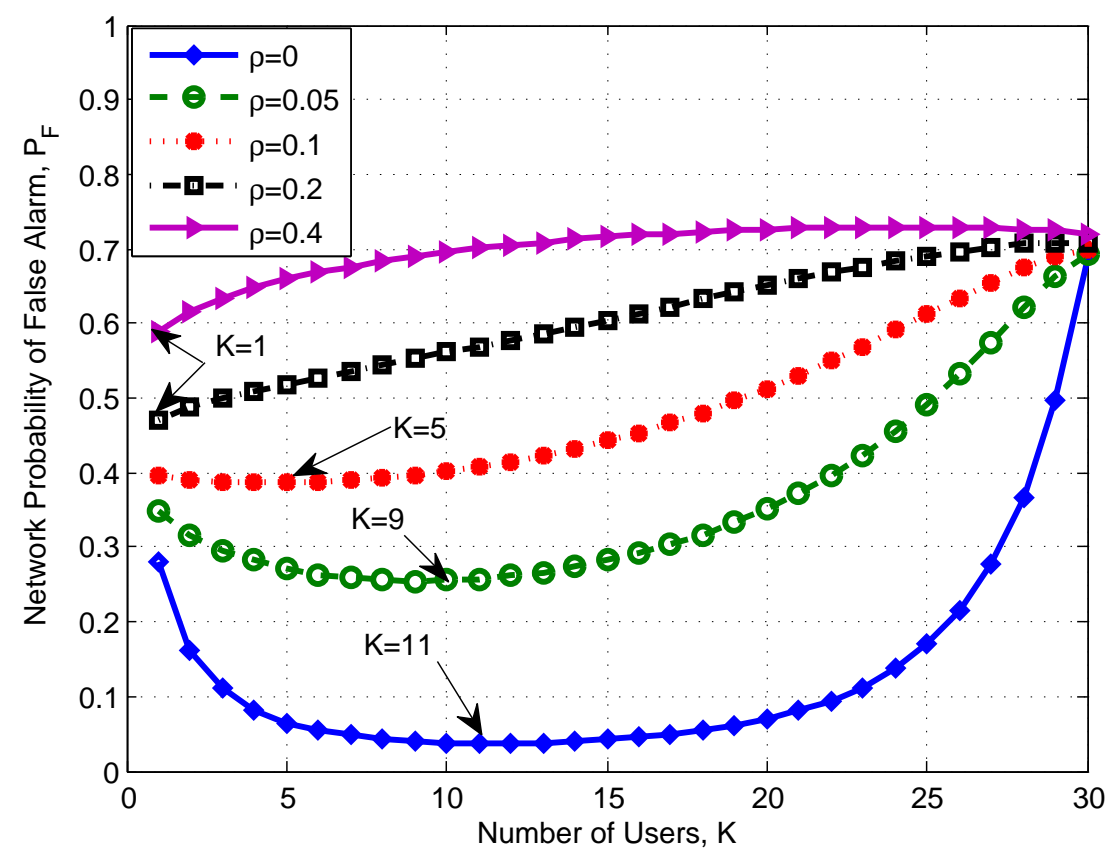

Figure 5.7: Network probability of false alarm versus number of users $K$, with $M=30$, SNR $=-10 \mathrm{~dB}$ and $P_{D}=0.9$.

In Figure 5.7, we plot the network probability of false alarm versus the number of users $K$ that are in favor of $H_{1}$ out of the $M$ cooperating users for $M=30$, SNR of $-10 \mathrm{~dB}$ and $P_{D}=0.9$. We show that there is a number of users $K$ that minimizes the network probability of false alarm for a given probability of detection, $P_{D}$. This value of $K$ changes with the correlation index $\rho$ as shown in the figure.

In Figure 5.8, we plot the network probability of false alarm versus the SNR for different number of cooperating users $K$ when $M=30$ and $P_{D}=0.9$, for $\rho=0.05$. We observe from the figure that the $K$-out-of- $M$ fusion rule outperforms the OR and AND fusion rules for low values of the SNR. Moreover, the number of users $K$ that minimizes $P_{F}$ is the same as the one obtained from Figure 5.7 for a certain correlation index. We also notice that, when SNR at the secondary user is high enough for the secondary user to make a correct detection on its own, cooperation becomes less effective and similar performance is observed for all the 


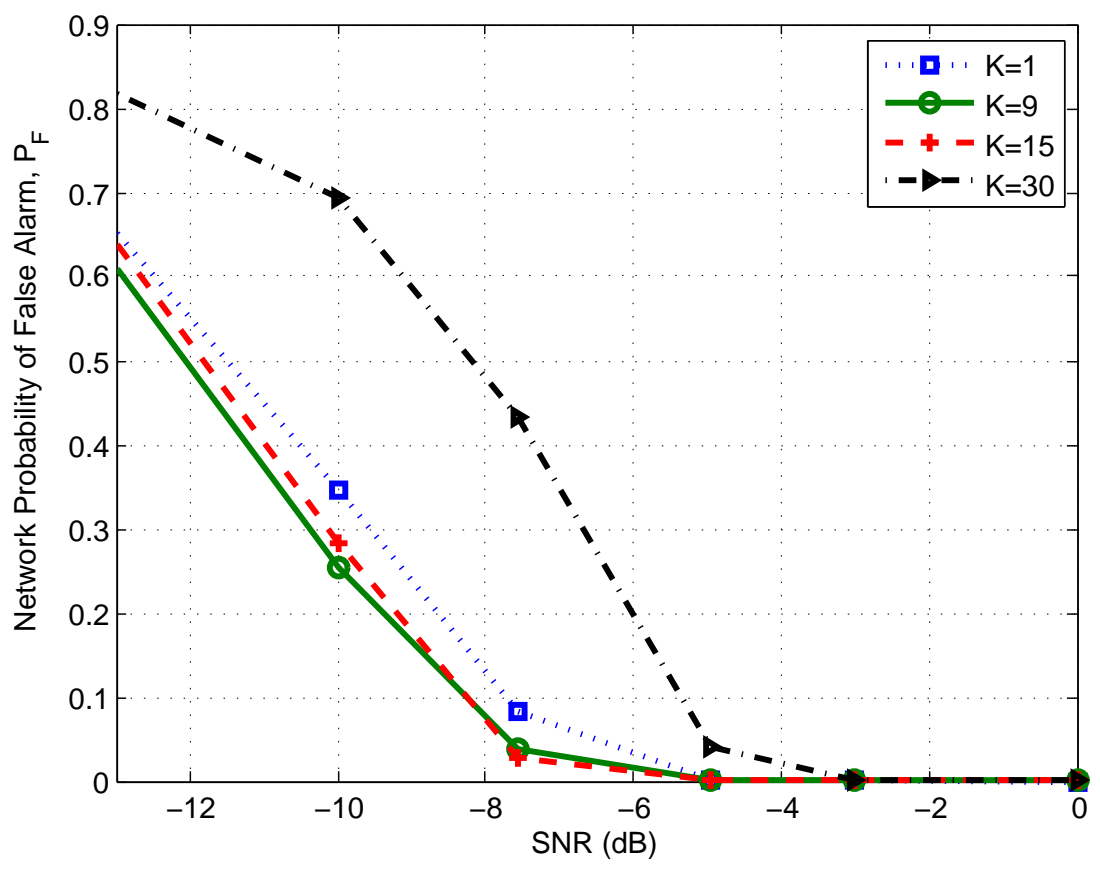

Figure 5.8: Network probability of false alarm versus SNR for different number of users $K$ with $\rho=0.05$ and $P_{D}=0.9$.

fusion rules considered.

In the remainder of this chapter, we employ the $K$-out-of- $M$ rule at the fusion center together with the correlation model described in section 5.3. We consider the more general case, where the false alarm rate is not fixed and the overall probability of sensing error is minimized at the fusion centre.

\subsection{Correlation-based $K^{*}$-out-of- $M$ Optimum Rule}

First, we show analytically that the number of users $K$ in the $K$-out-of- $M$ fusion rule that optimizes the network probability of false alarm (detection) is dependent on the local threshold $\gamma$ and the correlation index $\rho$. It is clear that both $P_{D}$ and $P_{F}$ are dependent on $\epsilon_{y}^{x}$ in (5.11). Since $P_{d, k}=P_{d}$ and $P_{f, k}=P_{f}$ are both dependent on $\gamma$ from (3.6) and (3.7) and 
assuming $\rho^{1}=\rho^{0}=\rho$, we can rewrite $\epsilon_{y}^{x}$ as follows:

$$
\epsilon_{y}^{x}=f(\gamma) \prod_{s=0}^{y-2} \frac{\rho(s+1-f(\gamma))+f(\gamma)}{1+s \rho}
$$

where $f(\gamma)$ represents a function of the local sensing threshold. Taking the logarithm of both sides, we get,

$$
\log \epsilon_{y}^{x}=\log f(\gamma)+\sum_{s=0}^{y-2} \log (\rho(s+1-f(\gamma))+f(\gamma))-\log (1+s \rho)
$$

Taking the derivative with respect to $s$ and rearranging the terms, we get,

$$
\frac{\partial \epsilon_{y}^{x}}{\partial s}=\rho \epsilon_{y}^{x} \sum_{s=0}^{y-2}\left(\frac{1}{\rho(s+1-f(\gamma))+f(\gamma)}-\frac{1}{1+s \rho}\right)
$$

To find the optimal value of $y$ that optimizes $\epsilon_{y}^{x}$, we set $\frac{\partial \epsilon_{y}^{x}}{\partial s}=0$. Assuming $\rho \neq 0$ and $\epsilon_{y}^{x} \neq 0$, we get,

$$
\sum_{s=0}^{y-2} \frac{1}{\rho(s+1-f(\gamma))+f(\gamma)}=\sum_{s=0}^{y-2} \frac{1}{1+s \rho} .
$$

Therefore, the optimal value of $K$, which is directly related to $y$ as seen from (5.13) and (5.14), is a function of both $\rho$ and $\gamma$ as shown in (5.25).

\subsubsection{Problem Formulation}

Motivated by the above reported results, we aim to find the optimal number of users $\left(K^{*}\right)$ and the optimum local sensing threshold $\left(\gamma^{*}\right)$ that jointly minimize the probability of sensing error at the fusion center subject to a limit on the probability of detection for a given correlation index, $\rho$. Using this sensing objective, we are jointly considering the sensing errors that occur when the channel is busy and idle, i.e., $P_{F}$ and $1-P_{D}$. Therefore, we formulate the problem as

$$
\underset{K, \gamma}{\arg \min } P_{E}(K, \gamma)=\left(1-P_{D}(K, \gamma)\right) P_{B}+P_{F}(K, \gamma) P_{I}
$$




$$
\begin{array}{ll}
\text { s. t. } & P_{D}(K, \gamma) \geq \beta \\
& K \in\{1, \ldots, M\}
\end{array}
$$

where $P_{E}$ is the probability of sensing error. The terms $P_{B}$ and $P_{I}$ are the prior probabilities that the primary user is present and absent on the channel, respectively. Those two terms will depend on the channel occupancy model described in section 3.3. The network probabilities of detection, $P_{D}$, and false alarm, $P_{F}$, for the $K$-out-of- $M$ rule are given in (5.13) and (5.14), respectively. Constraint (5.27) is used to guarantee a satisfactory level of protection for the primary users by setting a minimum limit, $\beta$, on the network probability of detection, $P_{D}$.

\subsubsection{Genetic Algorithm (GA) Based Solution}

The above optimization problem is a mixed integer non-linear programming (MINLP) problem which is generally $N P$-hard [91]. The formulated optimization problem constitutes simultaneously minimizing two conflicting non-linear objective functions subject to a lower limit on a non-linear function. Due to the complexity and non-linearity of the problem, we propose to use the genetic algorithm (GA) to solve the problem of minimizing the network probability of sensing error. GA is a general purpose optimization algorithm developed by Holland [134] that is well suited for multi-objective problems [117].

For a certain correlation index, $\rho$, the optimal values of $\gamma^{*}$ and $K^{*}$ are obtained using GA as follows:

Step 1 : Generate a population of $S$ chromosomes by randomly assigning values for $\gamma$ such that the constraint in (5.27) is satisfied. The value of $\gamma$ will depend on the desired local probability of false alarm, $P_{f, k}=P_{f}$, in (3.7).

Step 2 : Encode the value of $\gamma$ by representing them in a binary form.

Step 3 : Evaluate the fitness function for each of the chromosomes by converting $P_{E}$ in (5.26) to a function to be maximized for the encoded version of $\gamma$. 
Step 4 : Choose a number of chromosomes $S_{\text {best }}$, such that $0<S_{\text {best }}<S$, with the best fitness value (elite chromosomes) and place them directly in the next generation.

Step 5: Select $S-S_{\text {best }}$ parents from the entire population according to their fitness value by using the roulette wheel selection method where the probability of selecting a chromosome is equal to its fitness value normalized to the summation of the fitness values of all the chromosomes in the population.

Step 6 : Perform crossover and mutation on the selected chromosomes from Step 5 with probability, $p_{c}$ and $p_{m}$, respectively and obtain a new population of $S$ chromosomes.

Step 7 : Compute the fitness value for the new population. Terminate the algorithm if the budget of the fitness function evaluations is exhausted; otherwise, return to Step 1.

Step 8 : Repeat the above steps for $1 \leq K \leq M$.

Step 9 : Choose the value of $K$ that gives the maximum fitness value (minimum $P_{E}$ ), $K^{*}$.

The performance of GA depends greatly on the selection of parameters such as the population size, selection method, probabilities of crossover and mutation as well as the termination criteria. Although there is no complete agreement on the selection of those GA parameters, some ranges may provide a starting point for implementation. For standard bit implementation, the population size ranges from 20 to 100, the probability of crossover ranges from 0.6 to 0.95 and the probability of mutation ranges from 0.001 to 0.01 [135].

\subsection{Performance Evaluation}

In this section, we evaluate the performance in terms of the total probability of sensing error when using the $K$-out-of- $M$ fusion rule with correlated local decisions. Since the $K$-out-of- $M$ fusion rule with the optimal $K$ outperforms OR and AND fusion rules for weak correlation coefficients as shown before, we consider values of $\rho$ less than 0.1. In our evaluation, we 
use the following parameters: number of detection symbols $N=100, \mathrm{SNR}=-5 \mathrm{~dB}$, the number of secondary users $M=30$, the probability that the primary user is absent $P_{I}=0.3$, and the probability that the primary user is present $P_{B}=0.7$. We then use the genetic algorithm to find the optimal $\gamma^{*}$ that minimizes the probability of sensing error for each $K$ and compare $P_{E}\left(K, \gamma^{*}\right)$ to obtain the optimum $K^{*}$. Based on a number of test experiments, we choose the GA parameters listed in Table 5.1 that are well suited for our optimization problem.

Table 5.1: GA Parameters.

\begin{tabular}{|c|c|}
\hline Parameter & Value \\
\hline Initialization method & Random \\
\hline Selection process & Roulette wheel selection \\
\hline Crossover operation & One-point crossover \\
\hline Probability of crossover & 0.75 \\
\hline Probability of mutation & 0.002 \\
\hline Population size & 60 \\
\hline Number of elite chromosomes & 2 \\
\hline Termination criterion & 2000 evaluations \\
\hline
\end{tabular}

\subsubsection{Numerical Results}

First, we numerically evaluate the network probability of sensing error, $P_{E}$, with different local sensing threshold, $\gamma$. In Figure 5.9, we plot $P_{E}$ versus $\gamma$ for different number of cooperating secondary users $K$ when $\rho=0.05$. The figure shows that for each value of $K$, as $P_{E}$ varies with $\gamma$, there exists only one minimum value for $P_{E}$. This minimum value of $P_{E}$ will be different for different values of $\rho$. This relation can also be deduced from (5.25). The figure also shows that, for a certain value of of $\rho$, using the optimal pair $\left(K^{*}, \gamma^{*}\right)$, we can obtain a much lower $P_{E}$ when compared to using non-optimal settings. 


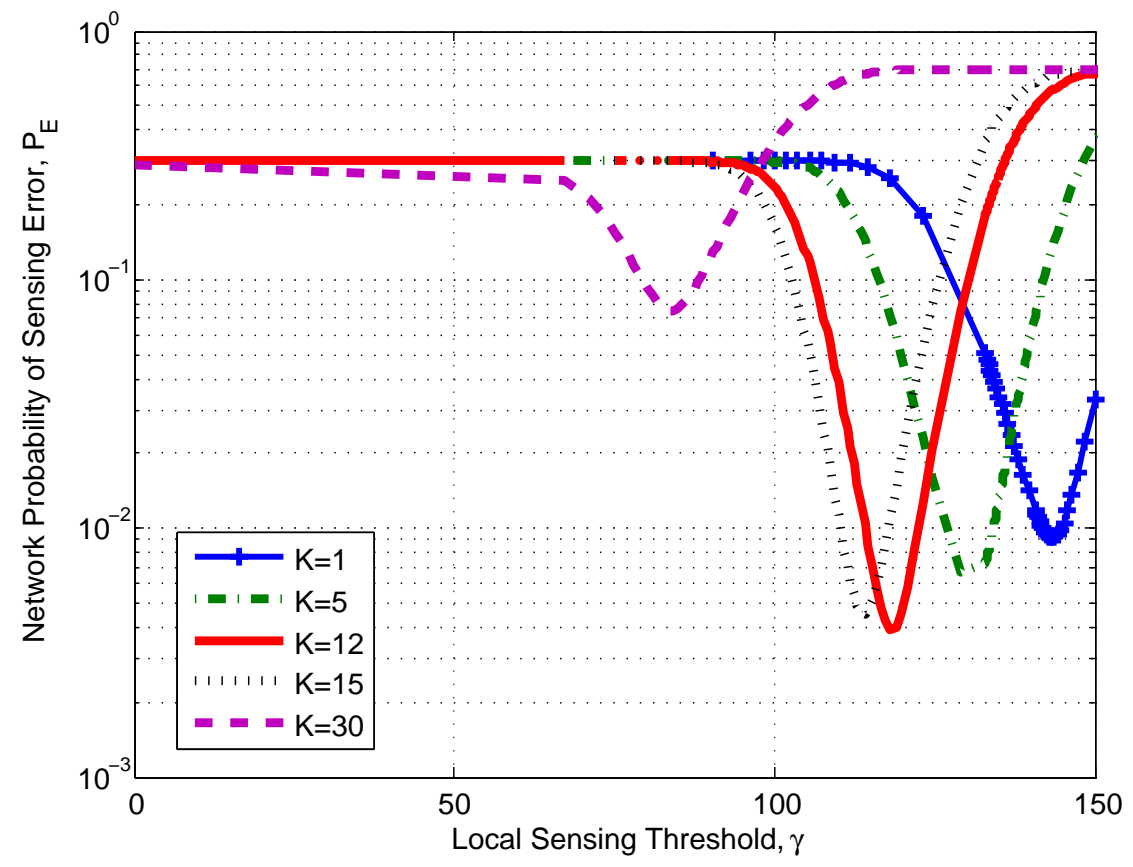

Figure 5.9: Network probability of sensing error versus local sensing threshold for different number of users $K$ when $\rho=0.05$.

\subsubsection{GA Based Results}

Next, we present the simulation results obtained by using GA to find the optimal pair $\left(K^{*}, \gamma^{*}\right)$ that minimizes $P_{E}$ for a given value of the correlation index $\rho$. Figure 5.10 shows $P_{E}$, calculated using the optimal threshold, $\gamma^{*}$, versus the number of users $K$, with $M=30$, for different $\rho$. The limit on $P_{D}, \beta$ in (5.27), is set to 0.9. We observe from the figure that, for $\rho<0.1$, the fusion rule that minimizes $P_{E}$ is the $K$-out-of- $M$ rule with the optimal number of users, $K^{*}$, depending on the correlation index $\rho$. However, as $\rho$ gets closer to 0.1, optimizing $K$ becomes less critical since similar performance can be obtained using the OR fusion rule. This observation agrees with the previous results presented in section 5.5.

In Figure 5.11, we plot the network probability of sensing error, $P_{E}$, with the optimal value of $\gamma^{*}$, for the MV, OR and AND fusion rules with different values of $\rho$ when $M=30$ and $\beta=0.9$. We notice from the figure that the network probability of sensing error degrades with the increase in the degree of correlation between secondary users for the three fusion 


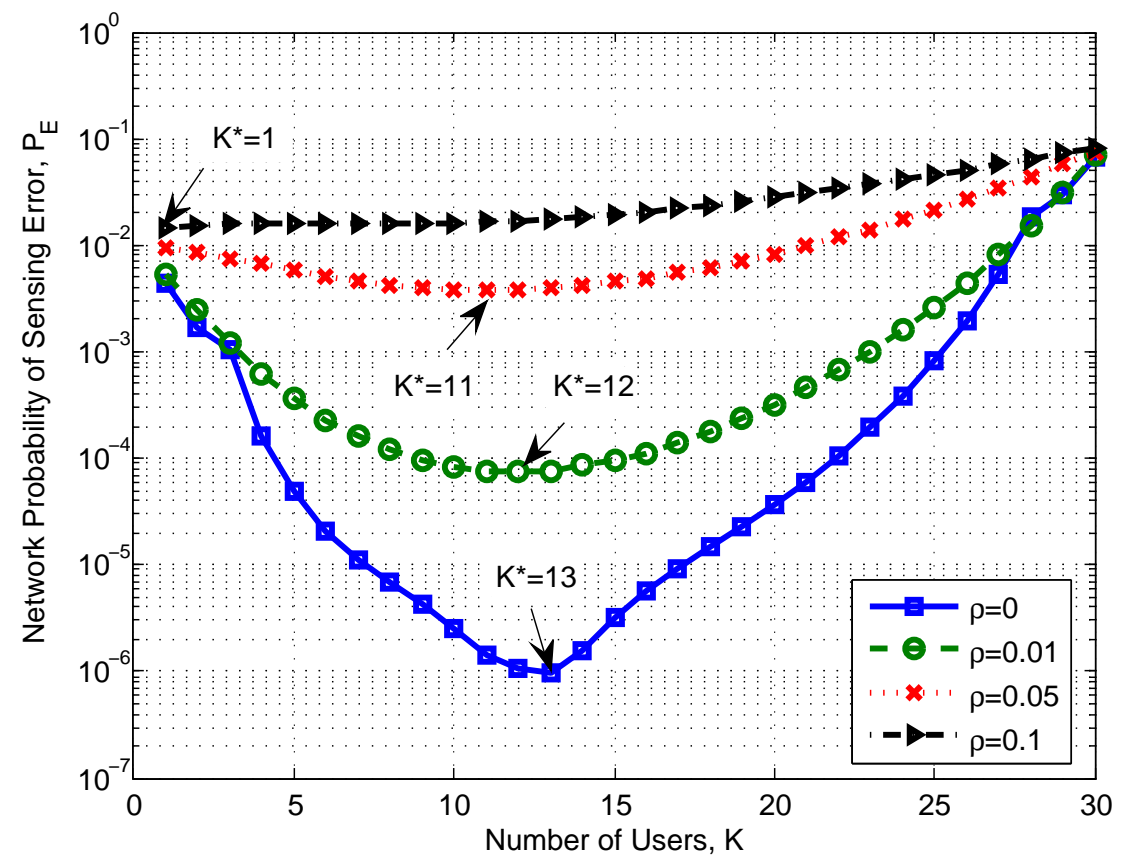

Figure 5.10: Network probability of sensing error versus the number of users $K$ when $\beta=0.9$.

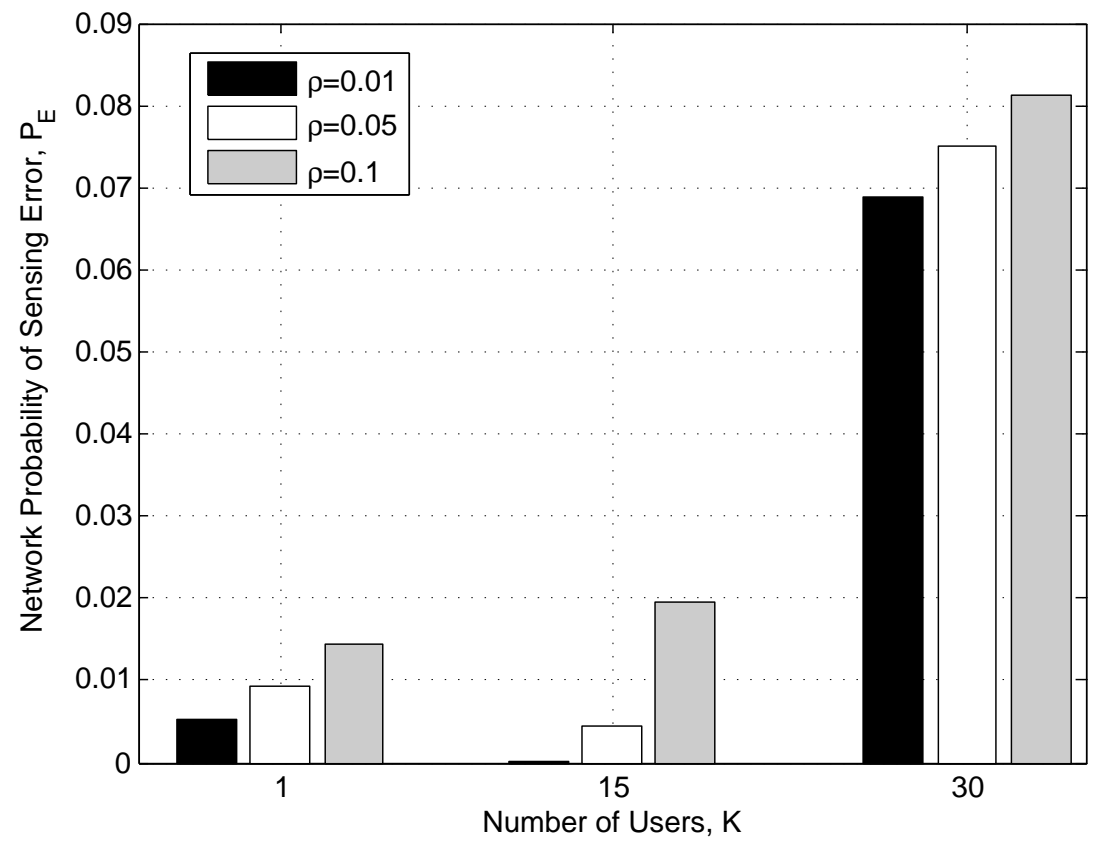

Figure 5.11: Network probability of sensing error versus the number of users $K$ for different $\rho$. 


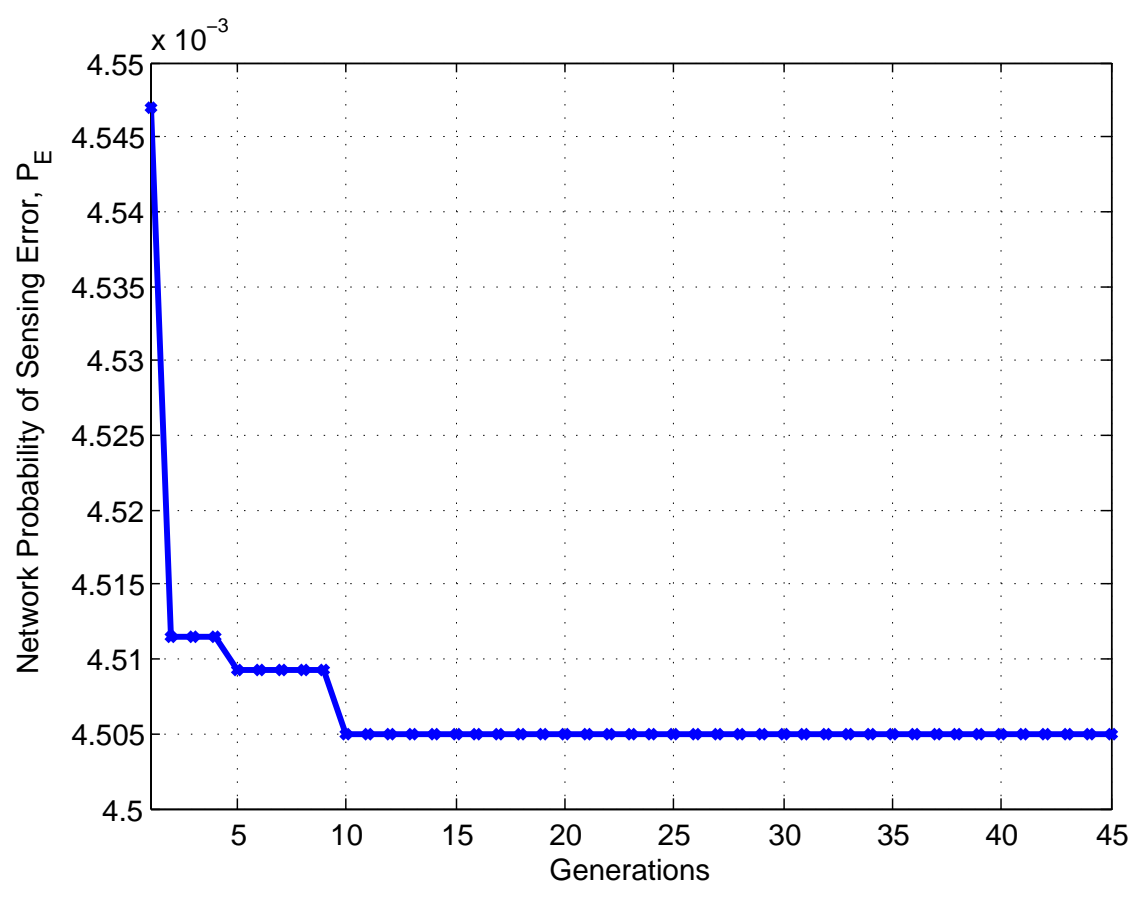

Figure 5.12: Evolution of the network probability of sensing error using GA algorithm when $K=11$ and $\rho=0.05$.

Table 5.2: Mean and Standard Deviation of the Network Probability of Sensing Error over 20 Runs.

\begin{tabular}{|c|c|c|c|c|c|}
\hline \multicolumn{2}{|c|}{} & $\mathrm{K}=1$ & $\mathrm{~K}=12$ & $\mathrm{~K}=15$ & $\mathrm{~K}=30$ \\
\hline \multirow{2}{*}{$\rho=0.01$} & Mean & 0.00538 & $7.1715 \times 10^{-5}$ & $9.1572 \times 10^{-5}$ & 0.06894 \\
\cline { 2 - 6 } & Standard deviation & $5.1012 \times 10^{-7}$ & $3.3325 \times 10^{-7}$ & $2.4072 \times 10^{-7}$ & $1.2228 \times 10^{-7}$ \\
\hline \multirow{2}{*}{$\rho=0.05$} & Mean & 0.00926 & 0.00385 & 0.00450 & 0.07515 \\
\cline { 2 - 6 } & Standard deviation & $1.3108 \times 10^{-5}$ & $3.3474 \times 10^{-6}$ & $2.2261 \times 10^{-6}$ & $6.2564 \times 10^{-7}$ \\
\hline \multirow{2}{*}{$\rho=0.1$} & Mean & 0.01429 & 0.01711 & 0.01957 & 0.08116 \\
\cline { 2 - 6 } & Standard deviation & $7.8609 \times 10^{-6}$ & $3.0690 \times 10^{-6}$ & $2.1175 \times 10^{-6}$ & $2.7098 \times 10^{-7}$ \\
\hline
\end{tabular}

rules considered. In Figure 5.12, we show the evolution of the network probability of sensing error, $P_{E}\left(K, \gamma^{*}\right)$, over 45 generations using the GA algorithm when $K=11$ and $\rho=0.05$. As seen from the figure, the GA algorithm for this case converges after 10 generations. The mean and standard deviation of the network probability of sensing error with optimal $\gamma^{*}$ over 20 runs for different number of users $K$ and different values of $\rho$ when $\beta$ is set to 0.9 are shown in Table 5.2. We observe from the table that the standard deviations of the function 
values for different $K$ and $\rho$ are relatively small which indicates that the solution quality of the GA algorithm is quite stable.

\subsection{Chapter Summary}

In this chapter, we studied the problem of cooperative spectrum sensing when the local decisions of the secondary users are correlated and the $K$-out-of- $M$ fusion rule is employed at the fusion center. We employed a correlation model, that uses a single parameter, $\rho$, to quantify the degree of correlation between secondary users' local decisions. We derived the system probabilities of detection and false alarm for the $K$-out-of- $M$ fusion rule and the special cases of the AND, OR and majority voting fusion rules. We used the NeymanPearson (NP) criterion to optimize the network probability of false alarm with a constraint on the network probability of detection when the local decisions are correlated. We showed that the detection performance of cooperative spectrum sensing degrades with the increase in the correlation between secondary local decisions for all the considered fusion rules. The performance difference between the three considered fusion rules is more pronounced for lower network probability of false alarm and lower SNR received at the secondary users. We also showed that there is an optimal value of $K$ that satisfies the NP criterion for each correlation index. For a certain correlation index ( $\rho<0.1$ in our simulations), the majority voting rule outperforms the AND and OR fusion rules. However, by optimizing the number

of cooperating secondary users, $K$, better performance than that achieved by the majority voting rule can be obtained. For $\rho>0.1$, the optimal fusion rule is the OR rule $(K=1)$.

We showed analytically that the number of users $K$ in the $K$-out-of- $M$ fusion rule that optimizes the network probability of false alarm (detection) is dependent on the sensing threshold and the correlation index, $\rho$. Motivated by this finding, we formulated the problem of minimizing the total probability of sensing error under the correlation model used in our analysis as a mixed integer non-linear programming (MINLP) problem which is generally 
NP-hard. Due to the complexity and non-linearity of the problem, we employed genetic algorithm to find the optimal local sensing threshold and optimal number of users for a certain correlation index. We showed that, for different values of the correlation index, the number of cooperating users that optimizes the performance in terms of minimizing the total probability of sensing error also differs. Therefore, when the correlation index is known at the fusion center, the optimum $K^{*}$-out-of- $M$ fusion rule can be employed at the fusion center to minimize the probability of sensing error.

So far in this thesis, we considered narrowband cooperative spectrum sensing, where each cooperating user can sense only a single channel during a sensing frame. As mentioned in Chapter 3, to increase the sensing efficiency of cooperative spectrum sensing, secondary users need to be able to sense more than one channel during each sensing period. Different from narrowband spectrum sensing, wideband spectrum sensing aims to find more spectral opportunities over a wide frequency range to achieve higher opportunistic aggregate throughput in cognitive radio networks. In the next chapter, we focus on wideband spectrum sensing using multiband joint detection, which employs an array of energy detectors, each of which detects one frequency band to efficiently utilize the wideband spectrum. We aim to improve the performance gain, in terms of the opportunistic throughput, for multiband joint detection by employing cooperation among secondary users. We further consider the effect of sensing errors due to time offset, which can be due to factors such as reporting delays or synchronization errors, on the performance of cooperative multiband joint detection. 


\section{Chapter 6}

\section{Multiband Cooperative Joint Detection with Sensing Errors}

\subsection{Introduction}

Conventional spectrum sensing techniques focus on sensing a single channel in each sensing round. Multiband sensing aims to find more spectral opportunities over a wide frequency range to achieve higher opportunistic aggregate throughput in cognitive radio networks. Narrowband sensing techniques cannot be directly used for performing multiband spectrum sensing because they make a single decision for the whole spectrum and therefore, cannot identify individual spectral opportunities that lie within the wideband spectrum. To sense multiple channels in each sensing round, secondary users can use parallel cooperative spectrum sensing techniques as discussed in Chapter 3 or they can perform spectrum sensing over a wide range of frequencies by scanning the spectrum or using multiple RF front ends for sensing multiple bands.

In multiband joint detection $[38,40,72,136,137]$, secondary users sense multiple narrowband channels jointly, instead of one channel at a time, by using an energy detector for each channel of interest. More specifically, the thresholds for the multiple narrowband energy 
detectors are jointly optimized to improve the aggregate opportunistic throughput of a cognitive radio system while limiting the interference to the primary users. Making decisions over multiple frequency bands jointly is essential for implementing efficient cognitive radio networks.

To exploit the spatial diversity of multiple secondary users performing multiband joint detection, cooperative wideband spectrum sensing schemes should be employed $[40,136]$. To this end, first, we investigate the performance of cooperative wideband spectrum sensing, based on multiband joint detection, when hard decision fusion is employed at the fusion center. With the objective of maximizing the opportunistic throughput while limiting the aggregate interference to primary users, we compare the achieved opportunistic throughput for non-cooperative multiband joint detection and multiband cooperative joint detection.

Next, we consider a probability-based combining scheme for cooperative spectrum sensing that takes into account the effect of sensing errors due to the time offset. This time offset can be due to reporting delay, synchronization issues or asynchronous reporting from cooperating users $[19,68]$. We investigate the performance of the multiband cooperative joint detection in the presence of time offset. By taking into consideration the statistical information of the licensed band occupancy, we derive the aggregate opportunistic throughput and the aggregate interference to primary users for cooperative spectrum sensing when sensing errors due to time offset are present.

\subsection{Related Work}

Wideband spectrum sensing systems are difficult to design, due to either high implementation complexity or high energy consumption from high rate analog-to-digital converter (ADC). Some approaches have appeared in the literature which offer the possibility of implementing wideband spectrum sensing. An early scheme for wideband spectrum sensing employed a tunable narrowband bandpass filter at the radio frequency (RF) front-end to 
search one narrow frequency band at a time [138], over which existing narrowband spectrum sensing techniques can be applied. The analysis in [139] showed that the detection of narrowband transmission using energy detection over multiband orthogonal frequency-division multiplexing (OFDM) is feasible, and can be further extended to cover more complex systems.

In [42], a wavelet-based spectrum sensing algorithm was proposed. In this algorithm, the power spectral density (PSD) of the wideband spectrum was modeled as a train of consecutive frequency subbands, where the PSD is smooth within each subband but exhibits discontinuities and irregularities on the border of two neighboring subbands. The wavelet transform was then used to locate the singularities of the wideband PSD, and the wideband spectrum sensing problem was formulated as a spectral edge detection problem. In [140], a filter bank detection approach was proposed that uses a bank of prototype filters (with different shifted central frequencies) to process the wideband signal. In each band, the corresponding portion of the spectrum for the wideband signal was down-converted to baseband and then low-pass filtered. Although this approach can capture the dynamic nature of wideband spectrum by using low sampling rates, the implementation of this approach requires a large number of RF components [141].

Compressive sensing based methods were introduced to implement wideband spectrum sensing in [43, 142-144]. Compressive sensing is a technique that can efficiently acquire a signal using relatively few measurements, by which unique representation of the signal can be found based on the signal's sparseness or compressibility in some domain. As the wideband spectrum is inherently sparse due to its low spectrum utilization, compressive sensing becomes a promising candidate to realize wideband spectrum sensing by using subNyquist sampling rates $[43,142]$. Despite its low sampling rate, an implementation issue of this approach is that a separate compression device is required in each sampling channel, and the synchronization of these devices must be addressed. Another problem is that the full spectrum reconstruction requires a high computational complexity, which results in a 
high spectrum sensing overhead.

The authors in $[38,40]$, introduced a novel multiband joint detection approach, which jointly detects the signal energy levels over multiple frequency bands, in order to improve the opportunistic throughput of secondary users and reduce their interference to the primary systems. Energy detection is used for multiband joint detection by employing an array of energy detectors, each of which detects one frequency band. In [72], the authors proposed a multiband sensing-time-adaptive joint detection framework for wideband spectrum sensing that collectively searches the secondary transmission opportunities over multiple frequency bands. The authors added periodic sensing to the system model used in [40] and considered adaptive selection of the sensing time. Based on the energy detector for narrowband sensing, both the sensing slot duration and detection thresholds for each narrowband detector were jointly optimized to maximize the achievable opportunistic throughput of the secondary network while keeping the interference to the primary network bounded to a reasonably low level.

Different from conventional cooperative spectrum sensing schemes that assume synchronous local sensing information, in $[68,145]$, the authors proposed a probability-based combining scheme that enables the combination of both synchronous and asynchronous sensing information from multiple secondary users by utilizing the statistics of the licensed band occupancy. In this chapter, we consider a probability-based combining scheme for cooperative spectrum sensing when each cooperating user is jointly detecting multiple bands simultaneously. This scheme takes into consideration both the detection errors and the time offset between local sensing observations and the final decision. In the next section, we present the multiband joint detection framework used in our analysis. 


\subsection{Multiband Cooperative Joint Detection}

We consider a wideband channel which is divided into $L$ non-overlapping narrowband subchannels and assume that a number of primary users $U^{\prime}$ share this spectrum. Specifically, a multicarrier modulation-based primary communication system is considered. Depending on the location and time, some of these subchannels might not be used by primary users and are available for secondary opportunistic transmission as illustrated in Figure 6.1.

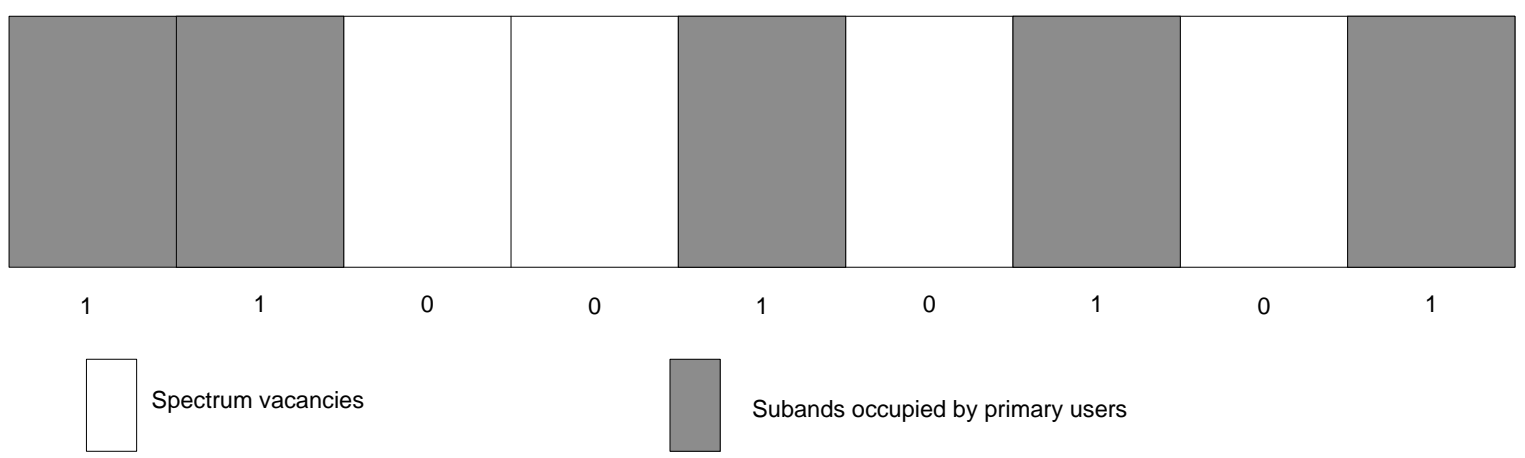

Figure 6.1: An illustration of the occupancy of a multiband channel.

We consider the alternating ON/OFF model for the primary user channel usage pattern as described in section 3.3. We are particularly interested in jointly detecting those vacant subchannels by $K$ cooperating secondary users. For each subchannel $l$, the signal is detected using the energy detection with $H_{0, l}$ and $H_{1, l}$ denoting, respectively, the hypothesis of the absence and presence of the primary users' transmission on the $l^{\text {th }}$ subchannel. Analog implementation of the energy detector, like the one shown in Figure 3.2, requires analog pre-filter with fixed bandwidth which becomes quite inflexible for simultaneous sensing of narrowband and wideband signals [146]. Digital implementation offers more flexibility by using fast Fourier transform (FFT) based spectral estimates, which allows sensing of multiple signals simultaneously. Multiband detection using L point FFT is illustrated in Figure 6.2.

In the frequency domain, the received signal at each subchannel can be represented by 


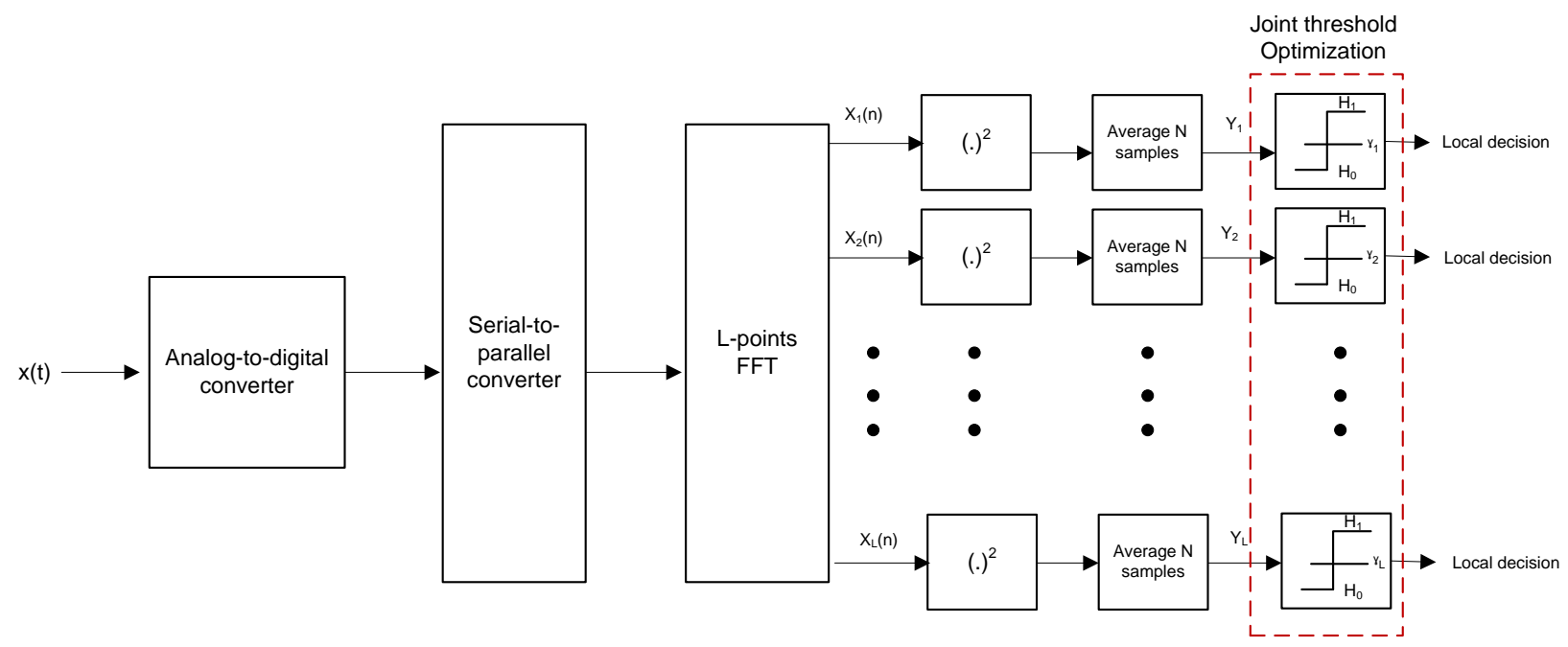

Figure 6.2: Block diagram of multiband joint detection for wideband spectrum sensing.

its discrete Fourier transform as [40]

$$
\begin{aligned}
& I_{l}: X_{l, k}(n)=V_{l, k}(n) \\
& B_{l}: X_{l, k}(n)=H_{l, k}^{\prime}(n) S_{l}(n)+V_{l, k}(n),
\end{aligned}
$$

where $B_{l}$ and $I_{l}$ indicate the presence and absence of the primary user's transmission on subchannel $l$ during the sensing time, respectively. The terms $S_{l}, H_{l, k}^{\prime}$ and $V_{l, k}$ denote, respectively, the PU signal component in the $l^{\text {th }}$ subchannel; the channel response between the transmitter of this PU signal and the $k^{\text {th }} \mathrm{SU}$; and the additive Gaussian noise with zero mean and variance $\sigma_{v}^{2}$. Without loss of generality, we assume that the transmitted signal, $S_{l}$, the channel gain, $H_{l, k}^{\prime}$, and the additive noise, $V_{l, k}$, are independent of each other.

We assume that the channel conditions between the primary and the secondary users remain unchanged during the detection interval [93]. The noise variance, $\sigma_{v}^{2}$, can easily be estimated by measuring the power level of a channel which is known to be idle and the channel coefficients, $H_{l, k}^{\prime}$, can also be obtained a priori when the primary transmitter is known for sure to be active $[40,72]$. This a priori information about the channel condition is attainable since pilot signals are transmitted periodically in the primary system for this 
specific purpose.

The test statistics, computed as the sum of received energy over $N$ samples, for the $k^{\text {th }}$ secondary user on the $l^{\text {th }}$ subchannel is given by

$$
Y_{l, k}=\sum_{n=0}^{N-1}\left|X_{l, k}(n)\right|^{2}
$$

According to the central limit theorem [82], for large $N$, the statistic, $Y_{l, k}$ is approximately normally distributed with mean, $\mathrm{E}\left[Y_{l, k}\right]$, and variance, $\operatorname{Var}\left[Y_{l, k}\right]$, given by

$$
\begin{gathered}
E\left[Y_{l, k}\right]= \begin{cases}N \sigma_{v}^{2}, & \text { if } P\left(I_{l}\right)=1 \\
N\left(1+\Gamma_{l, k}^{\prime}\right) \sigma_{v}^{2}, & \text { if } P\left(B_{l}\right)=1\end{cases} \\
\operatorname{Var}\left[Y_{l, k}\right]= \begin{cases}2 N \sigma_{v}^{4}, & \text { if } P\left(I_{l}\right)=1 \\
2 N\left(1+2 \Gamma_{l, k}^{\prime}\right) \sigma_{v}^{4}, & \text { if } P\left(B_{l}\right)=1\end{cases}
\end{gathered}
$$

where $\Gamma_{l, k}^{\prime}$ is the SNR of the primary signal at the $k^{\text {th }}$ secondary user for each subchannel $l$ and is given by

$$
\Gamma_{l, k}^{\prime}=\frac{\left|H_{l, k}^{\prime}\right|^{2} E\left[\left|S_{l}\right|^{2}\right]}{\sigma_{v}^{2}} .
$$

We assume that the transmitted signal in each subchannel has unit power, i.e., $E\left[\left|S_{l}\right|^{2}\right]=$ 1; this assumption holds when primary radios adopt uniform power transmission strategies given no channel knowledge at the transmitter side [38,40].

For each subchannel $l$, the probability of detection for the $k^{\text {th }}$ secondary user is given by

$$
\begin{aligned}
P_{d, k}^{l}\left(\gamma_{l, k}\right) & =P\left(Y_{l, k}>\gamma_{l, k} \mid B_{l}\right) \\
& =Q\left(\frac{\gamma_{l, k}-N\left(\sigma_{v}^{2}+\left|H_{l, k}^{\prime}\right|^{2}\right)}{\sqrt{2 N\left(\sigma_{v}^{2}+2\left|H_{l, k}^{\prime}\right|^{2}\right) \sigma_{v}^{2}}}\right)
\end{aligned}
$$

Similarly, the probability of false alarm for the $k^{\text {th }}$ secondary user on the $l^{\text {th }}$ subchannel 
is given by

$$
\begin{aligned}
P_{f, k}^{l}\left(\gamma_{l, k}\right) & =P\left(Y_{l, k}>\gamma_{l, k} \mid I_{l}\right) \\
& =Q\left(\frac{\gamma_{l, k}-N \sigma_{v}^{2}}{\sqrt{2 N \sigma_{v}^{4}}}\right)
\end{aligned}
$$

where $\gamma_{l, k}$ is the decision threshold of subchannel $l$ for the $k^{\text {th }}$ secondary user.

In multiband joint detection using cooperating secondary users, our objective is to find the optimal threshold for each subchannel $l$ such that those thresholds jointly maximize the aggregate opportunistic throughput of the secondary users while limiting the aggregate interference caused to primary users. The opportunistic throughput represents the throughput of the secondary users when they opportunistically use the licensed frequency bands that are not used by primary users. In this case, the optimal threshold on subchannel $l$ will depend on the thresholds for the other $L-1$ bands as shown in Figure 6.2. We consider cooperative spectrum sensing where the fusion center makes decisions about the occupancy of the multiple subchannels, based on the measurements collected from all the SUs over the subchannel of interest, using the OR fusion rule.

Considering the effect of sensing errors by cooperating secondary users (i.e., probability of miss-detection and false alarm) when jointly optimizing the decision thresholds for multiple channels, the network probabilities of detection and false alarm, $P_{D}^{l}$ and $P_{F}^{l}$, on each subchannel are respectively,

$$
P_{D}^{l}\left(\gamma_{l, k}\right)=1-\prod_{k=1}^{K}\left(1-P_{d, k}^{l}\right)
$$

and

$$
P_{F}^{l}\left(\gamma_{l, k}\right)=1-\prod_{k=1}^{K}\left(1-P_{f, k}^{l}\right) .
$$

If we consider cooperating secondary users with the same performance such that $\gamma_{l, k}=\gamma_{l}^{\prime}$, 
$P_{d, k}^{l}=P_{d}^{l}$ and $P_{f, k}^{l}=P_{f}^{l}, \forall k$, then,

$$
P_{D}^{l}\left(\gamma_{l}^{\prime}\right)=1-\left(1-P_{d}^{l}\right)^{K}
$$

and

$$
P_{F}^{l}\left(\gamma_{l}^{\prime}\right)=1-\left(1-P_{f}^{l}\right)^{K}
$$

\subsection{Sensing Errors due to Time Offset}

In the literature, it is usually assumed that the local observations of different users are obtained at the same time and sent to the fusion center without delay, which does not always hold in reality $[68,145]$. Therefore, in this section, we consider sensing errors due to time offset between local sensing observations performed by cooperating secondary users and the final sensing decision by the fusion center. This time offset can be caused by reporting delay, for example if SUs use random access to transmit on the control channel, synchronization issues or asynchronous reporting by secondary users. This time offset can result in imprecise spectrum information that may affect the achievable throughput by secondary users and may generate unacceptable interference to primary users. Therefore, we will use the statistical information about the primary user ON/OFF channel usage model described in section 3.3 to find the optimal decision thresholds for cooperative multiband detection in the presence of sensing errors due to time offset.

According to the local sensing performance, we can represent the local decision of each secondary user $k$ on subchannel $l, d_{l, k}$, assuming equal thresholds for all secondary users, $\gamma_{l}^{\prime}$, on subchannel $l$, as

$$
d_{l, k}=\left\{\begin{array}{c}
1, \quad Y_{l, k}>\gamma_{l}^{\prime} \\
0, \quad Y_{l, k} \leq \gamma_{l}^{\prime}
\end{array}\right.
$$

Since the primary user can appear at any time on the channel, the decision taken dur- 
ing the sensing time by cooperating secondary users may not necessarily reflect the actual presence or absence of the primary users' transmission when a final decision is made at the fusion center [68]. Therefore, in the presence of time offset, we can calculate the conditional probability that the $k^{t h}$ secondary user decides on the absence of the primary transmission, i.e., $d_{l, k}=0$, given $H_{x, l}, x \in\{0,1\}$ is the true state of the primary user's transmission when the fusion center makes a final decision as,

$$
P\left(d_{l, k}=0 \mid H_{x, l}\right)=P\left(d_{l, k}=0 \mid B_{l}\right) P\left(B_{l} \mid H_{x, l}\right)+P\left(d_{l, k}=0 \mid I_{l}\right) P\left(I_{l} \mid H_{x, l}\right),
$$

where the conditional probabilities $P\left(B_{l} \mid H_{x, l}\right)$ and $P\left(I_{l} \mid H_{x, l}\right)$ are the probabilities that the $l^{\text {th }}$ subchannel is sensed busy and idle, respectively, given $H_{x, l}, x \in\{0,1\}$ and

$$
P\left(B_{l} \mid H_{x, l}\right)+P\left(I_{l} \mid H_{x, l}\right)=1
$$

The sensing performance of the $k^{t h}$ secondary user at the time of sensing can be represented by

$$
P\left(d_{l, k}=0 \mid B_{l}\right)=1-P_{d, k}^{l}
$$

and

$$
P\left(d_{l, k}=0 \mid I_{l}\right)=1-P_{f, k}^{l} .
$$

From (3.8) and (3.9), we can calculate the conditional probabilities $P\left(B_{l} \mid H_{1, l}\right)$ and $P\left(I_{l} \mid H_{0, l}\right)$, respectively, as

$$
P\left(B_{l} \mid H_{1, l}\right)=\int_{\Delta t}^{\infty} f_{B}^{l}(t) d t=e^{-a_{l}(\Delta t)}
$$

and

$$
P\left(I_{l} \mid H_{0, l}\right)=\int_{\Delta t}^{\infty} f_{I}^{l}(t) d t=e^{-b_{l}(\Delta t)}
$$

where $f_{I}^{l}(t), f_{B}^{l}(t), a_{l}$ and $b_{l}$ are as defined in (3.8) and (3.9) for each subchannel $l$. The term 
$\Delta t$ is the time offset, which can be due to different factors as mentioned earlier, normalized to the total frame period $T$.

Therefore, we can calculate $P\left(d_{l, k}=0 \mid H_{x, l}\right)$, for $x=0$ and $x=1$, respectively, as

$$
P\left(d_{l, k}=0 \mid H_{0, l}\right)=\left(1-P_{d, k}^{l}\left(\gamma_{l}^{\prime}\right)\right)\left(1-e^{-b_{l}(\Delta t)}\right)+\left(1-P_{f, k}^{l}\left(\gamma_{l}^{\prime}\right)\right)\left(e^{-b_{l}(\Delta t)}\right),
$$

and

$$
P\left(d_{l, k}=0 \mid H_{1, l}\right)=\left(1-P_{d, k}^{l}\left(\gamma_{l}^{\prime}\right)\right) e^{-a_{l}(\Delta t)}+\left(1-P_{f, k}^{l}\left(\gamma_{l}^{\prime}\right)\right)\left(1-e^{-a_{l}(\Delta t)}\right)
$$

\subsubsection{Problem Formulation}

In this section, we consider the problem of finding the optimal thresholds for the $L$ subchannels such that they collectively maximize the aggregate opportunistic throughput for multiband cooperative joint detection subject to interference constraints for each primary user in the presence of time offset.

Let $D_{l}=\left[d_{l, 1}, d_{l, 2}, \cdots, d_{l, K}\right]$ denote the input local decision vector to the fusion center for each subchannel $l$, where $D_{l}$ can take $2^{K}$ possible realizations. Therefore, we can express the joint probability $P\left(D_{l} \mid H_{x, l}\right), x \in\{0,1\}$, at the fusion center for statistically independent secondary users decisions as,

$$
P\left(D_{l} \mid H_{x, l}\right)=\prod_{k=1}^{K} P\left(d_{l, k} \mid H_{x, l}\right) .
$$

We can express the expected opportunistic throughput on each subchannel $l$, using the OR fusion rule, as

$$
F_{l}\left(\gamma_{l}^{\prime}\right)=R_{l} \prod_{k=1}^{K} P\left(d_{l, k}=0 \mid H_{0, l}\right),
$$

where $R_{l}$ represents the achievable rate on each subchannel $l$ and $P\left(d_{l, k}=0 \mid H_{0, l}\right)$ is given by (6.19) for each secondary user $k$ making a decision on subchannel $l$.

Similarly, we can express the expected interference incurred by the primary user on each 
subchannel $l$ as

$$
\bar{A}_{l}\left(\gamma_{l}^{\prime}\right)=c_{l} \prod_{k=1}^{K} P\left(d_{l, k}=0 \mid H_{1, l}\right),
$$

where $c_{l}$ is the interference cost coefficient that assigns some relative interference protection priorities to different subchannels and $P\left(d_{l, k}=0 \mid H_{1, l}\right)$ is given by (6.20) for each secondary user $k$ making a decision on subchannel $l$.

Therefore, we can express the aggregate opportunistic throughput, $F$, for the secondary users as

$$
F=\sum_{l=1}^{L} F_{l}
$$

Similarly, we can express the aggregate interference to the $u^{\text {th }}$ primary user, $A_{u}$, as

$$
A_{u}=\sum_{l \in S_{l, u}^{\prime}} \bar{A}_{l}, \quad u=1, \cdots, U^{\prime}
$$

where $S_{l, u}^{\prime}$ is the set of subchannels that primary user $u$ is using out of the total $L$ subchannels.

As such, we can formulate the optimization problem of maximizing the opportunistic throughput for multiband cooperative joint detection in the presence of time offset as

$$
\arg _{\left[\gamma_{1}^{\prime} \ldots \gamma_{L}^{\prime}\right]} \max F
$$

s. t.

$$
\begin{gathered}
A_{u} \leq \varepsilon_{u}, \quad u=1, \cdots, U^{\prime} \\
\mathbf{1}-\boldsymbol{P}_{\boldsymbol{D}} \leq \boldsymbol{\alpha} \\
\boldsymbol{P}_{\boldsymbol{F}} \leq \boldsymbol{\beta}^{\prime}
\end{gathered}
$$

where constraint (6.27) puts a limit, $\varepsilon_{u}$, on the aggregate interference that can be tolerated by the $u^{\text {th }}$ primary user. For a single-user primary system where all the subchannels are used by one primary user, we have $U^{\prime}=1$. Constraint (6.28) limits the probability of miss-detection 
on each subchannel with $\boldsymbol{\alpha}=\left[\alpha_{1}, \cdots, \alpha_{L}\right]^{T}$ to guarantee a certain protection for primary users on each subchannel. Constraint (6.29) limits the probability of false alarm on each subchannel with $\boldsymbol{\beta}^{\prime}=\left[\beta_{1}^{\prime}, \cdots, \beta_{L}^{\prime}\right]^{T}$ to guarantee a minimum requirement on the spectrum utilization by secondary users. In a vector-based format, the probabilities of detection and false alarm on all subchannels are represented, respectively, as

$$
\boldsymbol{P}_{\boldsymbol{D}}=\left[P_{D}^{1}\left(\gamma_{1}^{\prime}\right), P_{D}^{2}\left(\gamma_{2}^{\prime}\right), \cdots, P_{D}^{L}\left(\gamma_{L}^{\prime}\right)\right]^{T}
$$

and

$$
\boldsymbol{P}_{\boldsymbol{F}}=\left[P_{F}^{1}\left(\gamma_{1}^{\prime}\right), P_{F}^{2}\left(\gamma_{2}^{\prime}\right), \cdots, P_{F}^{L}\left(\gamma_{L}^{\prime}\right)\right]^{T}
$$

As mentioned earlier, we consider cooperating secondary users with same detector performance, such that, $P_{d, k}^{l}=P_{d}^{l}$ and $P_{f, k}^{l}=P_{f}^{l}$, therefore,

$$
F_{l}\left(\gamma_{l}^{\prime}\right)=R_{l}\left(P\left(d_{l, k}=0 \mid H_{0, l}\right)\right)^{K}
$$

and

$$
\bar{A}_{l}\left(\gamma_{l}^{\prime}\right)=c_{l}\left(P\left(d_{l, k}=0 \mid H_{1, l}\right)\right)^{K}
$$

The optimization problem in (6.26)-(6.29) is a non-linear optimization problem. However, the fact that the $Q$-function is monotonically non-increasing allows us to transform the constraints in (6.28) and (6.29) into linear constraints $[38,40]$. Therefore, we can reformulate the optimization problem as

$$
\arg _{\left[\gamma_{1}^{\prime} \ldots \gamma_{L}^{\prime}\right]} \max F
$$

s. t.

$$
\begin{gathered}
A_{u} \leq \varepsilon_{u}, \quad u=1, \cdots, U^{\prime} \\
\gamma_{\min , l} \leq \gamma_{l}^{\prime} \leq \gamma_{\max , l}, \quad l=1, \cdots, L
\end{gathered}
$$

where $\gamma_{\min , l}$ and $\gamma_{\max , l}$ are the lower and upper bounds on the threshold of each subchannel, 
respectively.

\subsubsection{Derivation of Threshold Bounds}

In this section, we derive the threshold bounds, $\gamma_{\min , l}$ and $\gamma_{\max , l}$ by using the constraints on the network probabilities of detection, $P_{D}^{l}$, and false alarm, $P_{F}^{l}$, for each subchannel, such that,

$$
\begin{gathered}
1-P_{D}^{l} \leq \alpha_{l} \quad l=1, \cdots, L \\
P_{F}^{l} \leq \beta_{l}^{\prime} \quad l=1, \cdots, L
\end{gathered}
$$

For the hard combining scheme using the OR fusion rule, we can express the bounds on the thresholds for each user $k$ on each subchannel $l$, using the limit on $P_{D}^{l}$, as

$$
\left(1-P_{d}^{l}\right)^{K} \leq \alpha_{l} \quad l=1, \cdots, L
$$

By substituting (6.39) into (6.6) and taking into account the fact that $Q(x)$ is a monotonically non-increasing function of $x$, we get

$$
\gamma_{l}^{\prime} \leq \gamma_{\max , l} l=1, \cdots, L
$$

where,

$$
\gamma_{\max , l} \triangleq\left(Q^{-1}\left(1-\sqrt[K]{\alpha_{l}}\right)\right) \sqrt{2 N\left(\sigma_{v}^{2}+2\left|H_{l}^{\prime}\right|^{2}\right) \sigma_{v}^{2}}+N\left(\sigma_{v}^{2}+\left|H_{l}^{\prime}\right|^{2}\right)
$$

where $H_{l}^{\prime}$ is the channel gain for subchannel $l$.

Similarly, to obtain the lower bound for each user $k$ on each subchannel $l, \gamma_{\min , l}$, we use the limit on $P_{F}^{l}$. Therefore, from (6.38) and (6.9), we have

$$
P_{f}^{l} \leq 1-\sqrt[K]{1-\beta_{l}^{\prime}}
$$


By substituting (6.42) into (6.7), we get

$$
\gamma_{l}^{\prime} \geq \gamma_{\min , l} l=1, \cdots, L,
$$

where,

$$
\gamma_{m i n, l} \triangleq\left(Q^{-1}\left(1-\sqrt[K]{1-\beta_{l}^{\prime}}\right)\right) \sqrt{2 N \sigma_{v}^{4}}+N \sigma_{v}^{2} .
$$

\subsection{Performance Evaluation}

In this section, we numerically evaluate the performance of the proposed cooperative multiband sensing scheme. We consider a multiband single primary user system $\left(U^{\prime}=1\right)$ with 8 subchannels and each subchannel has a bandwidth of $6 \mathrm{MHz}$. For each subchannel $l$, the probability of miss-detection of the primary user is at most $\alpha_{l}=0.1$ and the probability of opportunistic spectrum utilization is at least $50 \%$, i.e., $\beta_{l}^{\prime}=0.5$. The number of cooperating users, $K$, is equal to two, the number of detection symbols, $N$, is equal to 100 and the noise variance, $\sigma_{v}^{2}$, is equal to one. The subchannel parameters, which are the achievable opportunistic throughput, $R_{l}$, the cost of interfering with the primary user, $c_{l}$, and the subchannel gain, $H_{l}^{\prime}$, are generated randomly for each subchannel.

In Figure 6.3, we plot the maximum aggregate opportunistic throughput, $F$, which is obtained by solving the optimization problem in (6.34)-(6.36) for the optimal values of the threshold for each subchannel, $\gamma_{l}^{\prime}$, for different values of the aggregate interference, A. For multiband joint detection, we compare the cooperative scheme using the OR fusion rule with the non-cooperative scheme (NCS) [38]. From the figure, it is clear that the cooperative scheme outperforms the non-cooperative scheme in terms of the aggregate opportunistic throughput for a certain aggregate interference. For example, the cooperative scheme provides approximately $18 \%$ increase in throughput compared to the non-cooperative scheme when the aggregate interference $A=1.5$. We also notice that the maximum achievable throughput increases as the limit on the interference tolerated by the primary user, $\varepsilon$ in 
(6.35), increases, for both the cooperative and non-cooperative schemes.

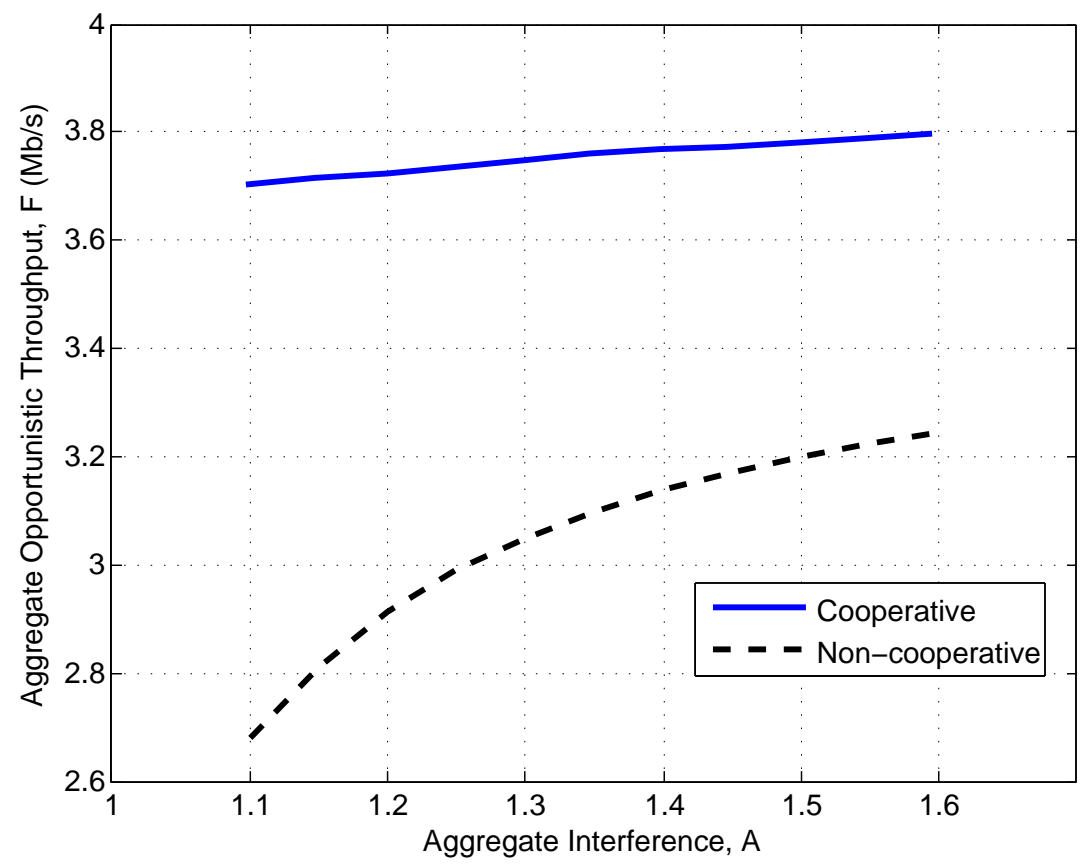

Figure 6.3: Comparision of maximum aggregate opportunistic throughput for the cooperative and non-cooperative schemes.

In Figures 6.4 and 6.5, the network probability of false alarm $P_{F}^{l}$ and the network probability of miss $P_{M}^{l}$ are plotted, respectively, for each subchannel $l$ for the cooperative and the non-cooperative schemes when limiting the interference to the primary user to $\varepsilon=1.25$. We can see from Figure 6.4 that better channel utilization, $1-P_{F}^{l}$, is obtained when using the OR fusion rule compared to the non-cooperative scheme. From Figure 6.5, we can see that the network probability of miss-detection, $P_{M}^{l}=1-P_{D}^{l}$, for each subchannel, $l$, is bounded by $\alpha_{l}=0.1$ for both schemes. This is an important constraint since it guarantees the desired protection for the primary user while maximizing the aggregate opportunistic throughput.

In Figure 6.6, we plot the maximum aggregate opportunistic throughput, $F$, as a function of the average SNR. The maximum aggregate opportunistic throughput that can be achieved is constrained by the limit on the aggregate interference $\varepsilon=2$. Figure 6.6 shows that as the SNR increases, the opportunistic throughput increases for both the cooperative and 


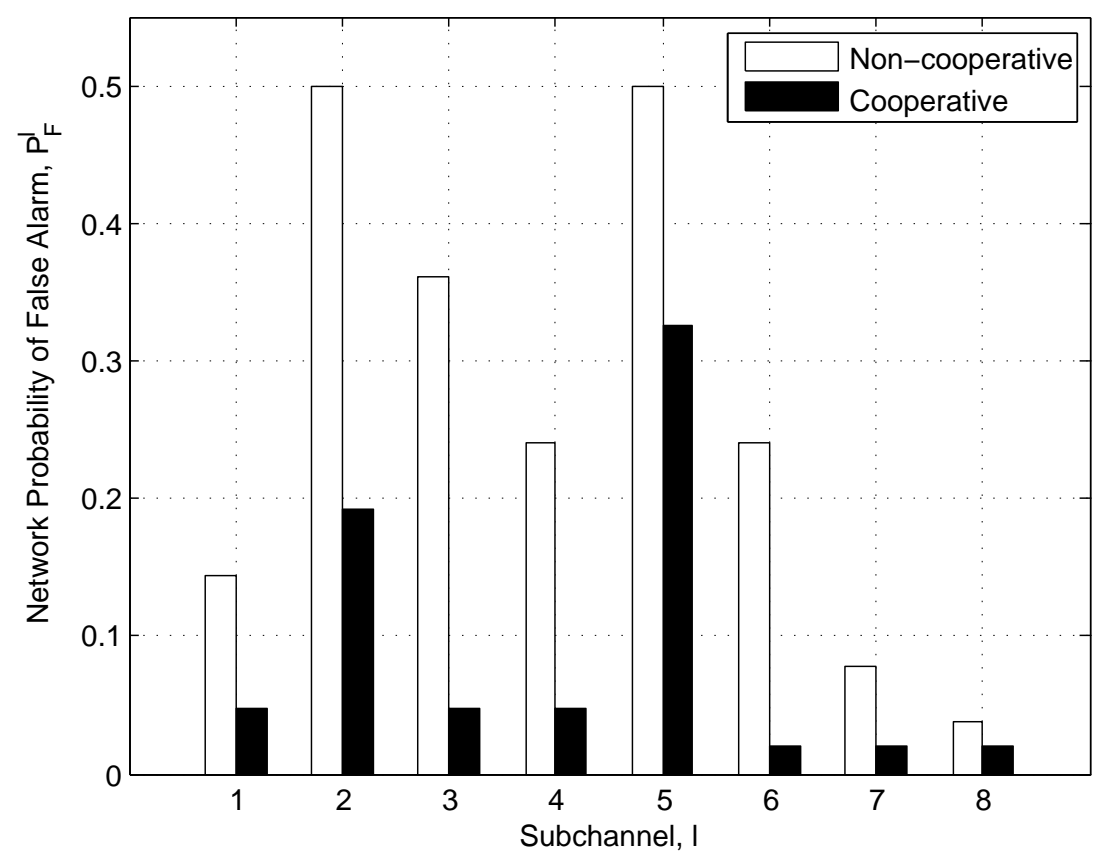

Figure 6.4: Network probability of false alarm for each subchannel $l$ for $\varepsilon=1.25$.

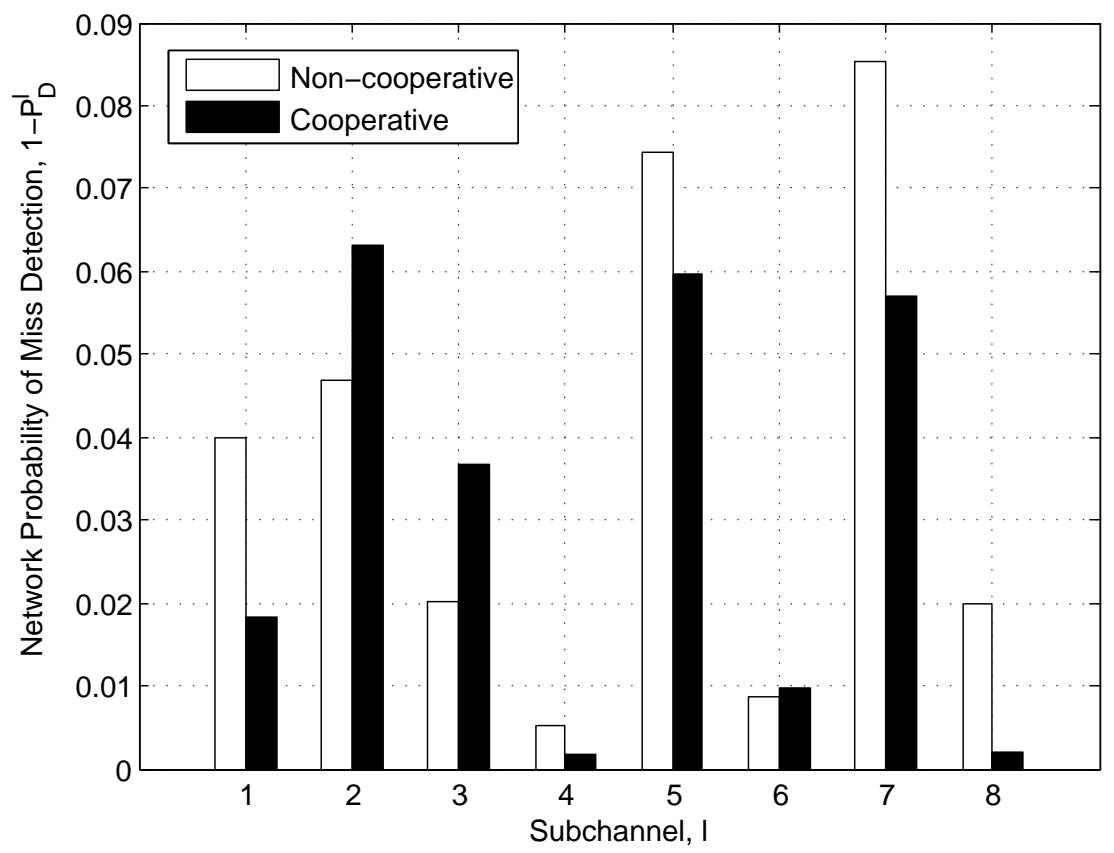

Figure 6.5: Network probability of miss-detection for each subchannel $l$ for $\varepsilon=1.25$.

non-cooperative schemes. For low average SNR, the cooperative scheme outperforms the non-cooperative scheme. However, as SNR approaches $0 \mathrm{~dB}$, the SNR at the secondary user 


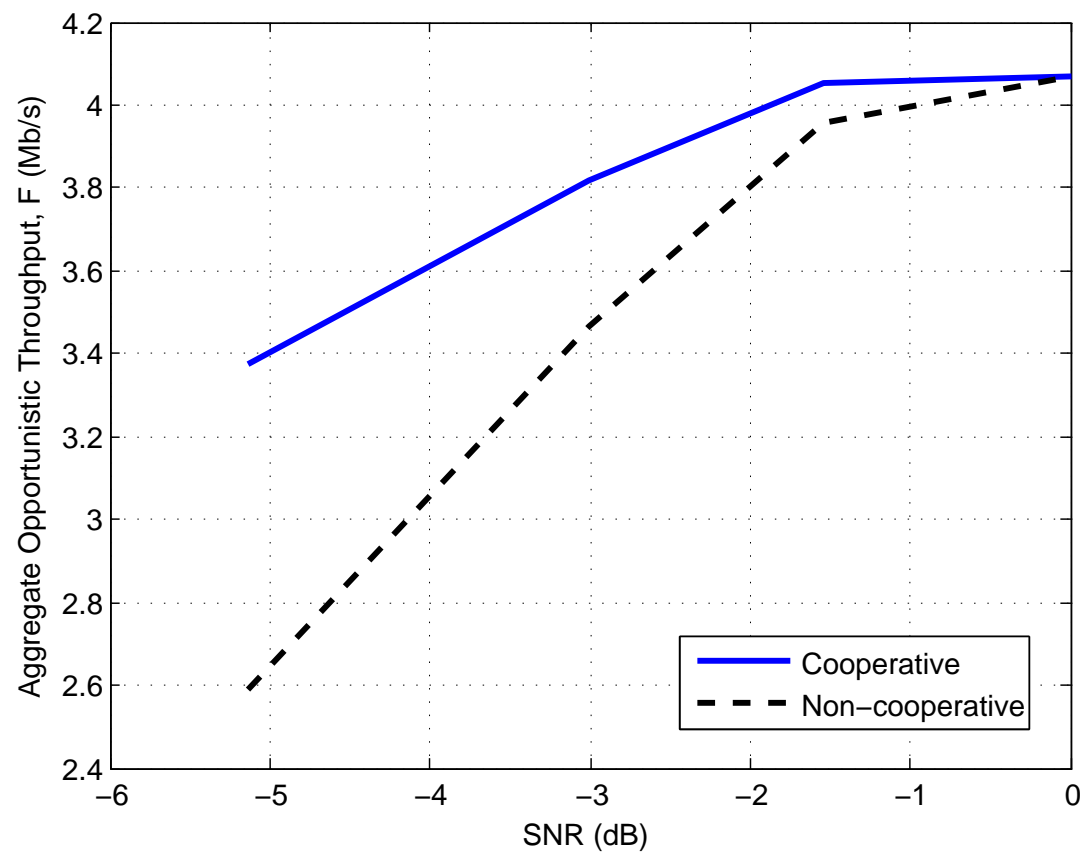

Figure 6.6: Maximum aggregate opportunistic throughput versus average SNR for $\varepsilon=2$.

is high enough for the secondary user to make a correct detection on its own. In this case, cooperation becomes less effective and almost the same maximum opportunistic throughput can be achieved for both schemes.

Next, we investigate the effect of sensing errors due to time offset on the achievable aggregate opportunistic throughput by cooperative multiband joint detection. Using the exponential ON/OFF model, we generate $1 / a_{l}$ and $1 / b_{l}$, the mean duration of the ON and OFF periods for each subchannel $l$, respectively, by using uniform distribution between $[0.5$, 5.5] for each subchannel [83].

In Figure 6.7, we plot the maximum aggregate opportunistic throughput, $F$, versus the aggregate interference, $A$, for different values of the normalized time offset, $\Delta t$. From the figure, it is clear that for the same limit of interference tolerated by the primary user, the maximum achievable aggregate throughput decreases as $\Delta t$ increases.

In Figures 6.8 and 6.9, the network probability of false alarm, $P_{F}^{l}$, and the network probability of miss-detection, $P_{M}^{l}=1-P_{D}^{l}$, are plotted respectively for each subchannel $l$ for 


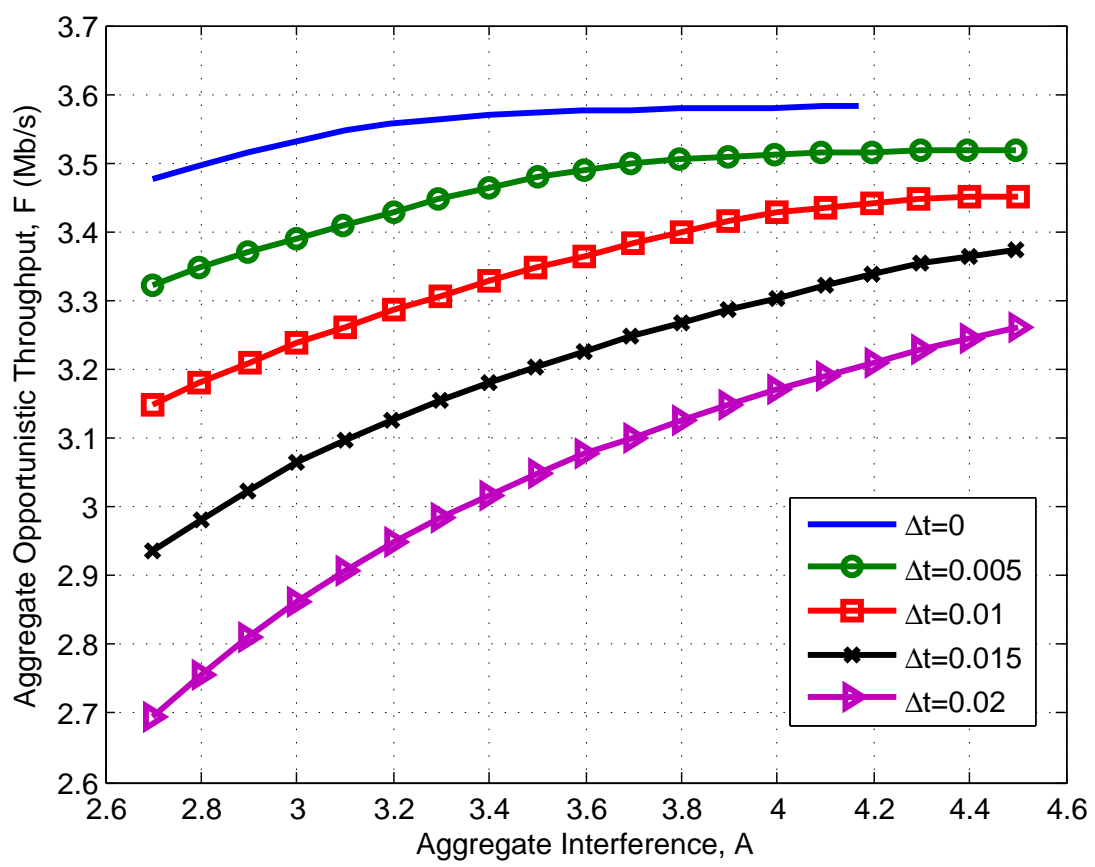

Figure 6.7: Maximum aggregate opportunistic throughput versus the aggregate interference for different normalized time offsets.

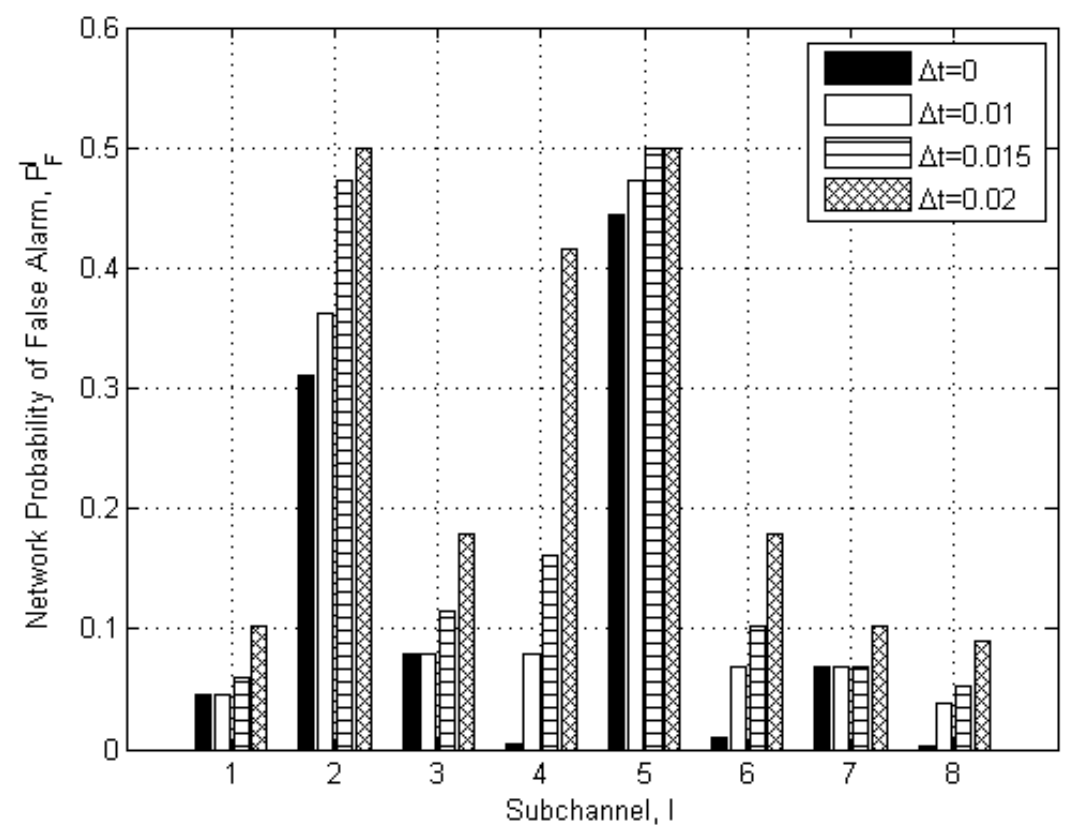

Figure 6.8: Network probability of false alarm for each subchannel $l$ for different normalized time offsets and $\varepsilon=4$. 


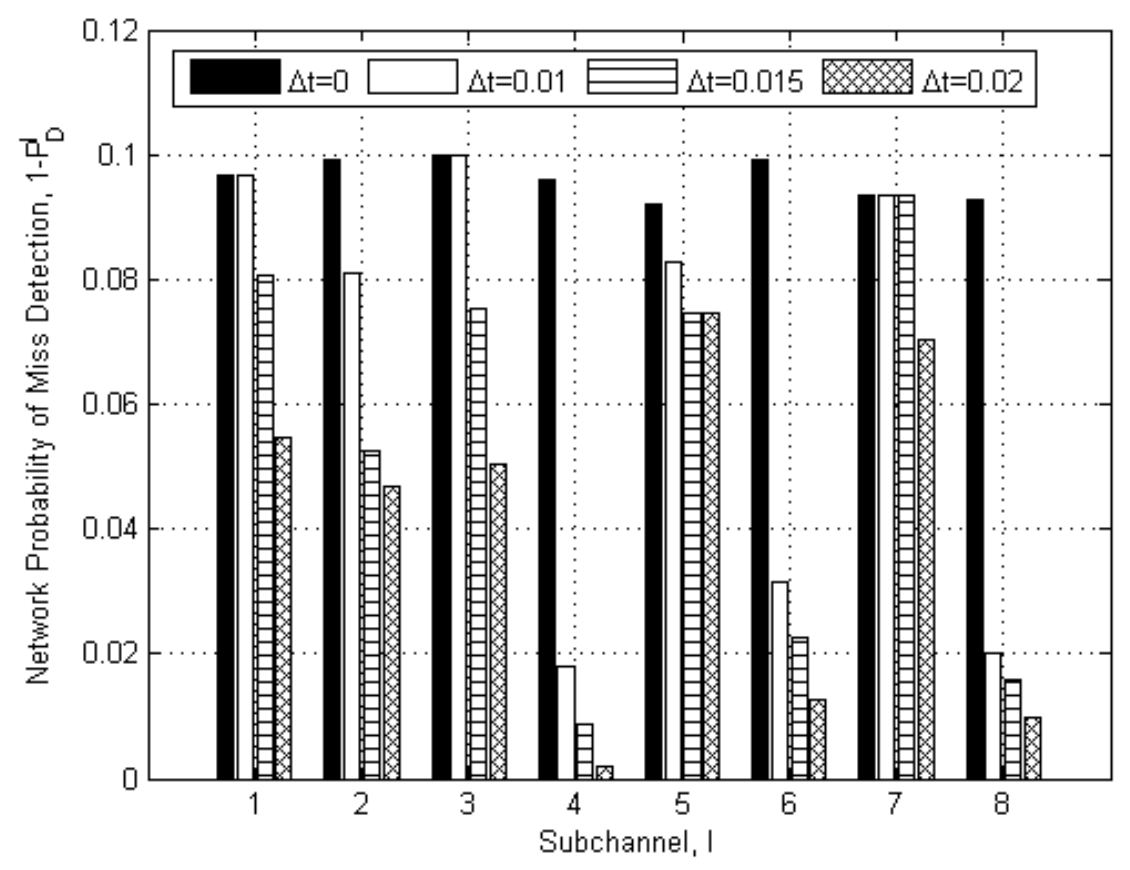

Figure 6.9: Network probability of miss-detection for each subchannel $l$ for different normalized time offsets and $\varepsilon=4$.

different values of $\Delta t$ when limiting the interference to the primary user to $\varepsilon=4$. Although the performance of each subchannel will depend on its gain, as we can see from Figure 6.8, when $\Delta t$ increases, $P_{F}^{l}$ increases as well for all subchannels. This increase in $P_{F}^{l}$ will result in lower values of the optimal decision thresholds and consequently lower aggregate opportunistic throughput. From Figure 6.9, it is clear that the the network probability of miss-detection, $P_{M}^{l}$ for each subchannel is bounded by $\alpha_{l}=0.1$ for all values of the normalized time offset, $\Delta t$. This bounded value of $P_{M}^{l}$ guarantees the desired protection for the primary user regardless of the value of $\Delta t$.

\subsection{Chapter Summary}

To increase the sensing efficiency of spectrum sensing techniques, secondary users need to be able to detect multiple opportunities during a sensing period without degrading the 
sensing accuracy. Multiband joint detection is one approach for wideband spectrum sensing that allows secondary users to detect multiple bands simultaneously with jointly optimized local thresholds. In this chapter, we employed hard decision cooperation between secondary users performing multiband joint detection in order to improve the spectrum utilization of secondary users and reduce interference to the primary users. To account for possible sensing errors due to time offset resulting from factors such as reporting delay, asynchronous sensing or synchronization errors, we employed a probability-based combining scheme for combining the decisions of secondary users when each cooperating user is jointly detecting multiple bands simultaneously. By considering the primary user's spectrum usage model, we derived the opportunistic throughput and interference to primary users on each subchannel in the presence of time offset. We then formulated the problem of maximizing the aggregate opportunistic throughput for the cooperative multiband joint detection subject to a limit on the aggregate interference to primary users.

We numerically evaluated the performance of cooperative multiband joint detection, using the OR fusion rule, and compared it with the non-cooperative scheme. We showed that cooperative sensing is effective in improving the aggregate opportunistic throughput of secondary users while limiting the interference to primary users as compared to the noncooperative scheme. This performance improvement is more pronounced at low received SNR at the secondary users. We also evaluated the performance of multiband cooperative joint detection in the presence of time offset. Numerical results showed that as the time offset increases, the probability of false alarm on each subchannel also increases which results in lower channel utilization. Results also showed that, for certain limit on the aggregate interference to primary users, the sensing errors due to time offset lead to performance degradation in terms of the aggregate opportunistic throughput. 


\section{Chapter 7}

\section{Conclusions and Future Work}

The ever-increasing demand for higher data rates in wireless communications in the face of limited or under-utilized spectral resources has motivated the introduction of dynamic spectrum access (DSA). Traditionally, licensed spectrum is allocated to licensed users and is intended to be used only by those users. The concept behind DSA is to allow secondary users to exploit these under-utilized spectral resources by reusing unused spectrum in an opportunistic manner without causing harmful interference to the primary users of the spectrum. To achieve this goal, secondary users, equipped with cognitive radios, must sense the spectrum to detect its availability and must be able to detect very weak primary users' signals. Therefore, spectrum sensing plays a crucial role in the successful deployment of cognitive radio networks. To further improve the spectrum sensing performance, efficient cooperative spectrum sensing schemes, that exploits multiuser diversity, need to be employed. In most of the previous work, sequential sensing is employed for cooperative spectrum sensing wherein the same set of cooperating users sense a single channel in each sensing period to increase the sensing accuracy. However, this approach greatly limits the sensing efficiency and can result in a large cooperation overhead. In this thesis, we considered more efficient cooperative spectrum sensing techniques and proposed efficient solutions both at the local sensing level and fusion level to best leverage the achievable cooperative gain. 


\subsection{Conclusions}

In this thesis, we focused on the design of efficient cooperative spectrum sensing techniques for cognitive radio networks to increase the achievable cooperative gain while limiting the incurred cooperation overhead and sensing errors. To this end, we studied two sensing schemes that enables the sensing of multiple channels in each sensing period, namely, the parallel cooperative spectrum sensing scheme and the multiband joint detection scheme. We mainly focused in our study on two important design issues for cooperative spectrum sensing which are cooperative user selection techniques and reliable decision fusion schemes in the presence of practical limitations.

In Chapter 2, we reviewed the most common spectrum sensing techniques for cognitive radio networks and detailed their advantages and disadvantages. To address the limitations of the spectrum sensing techniques by a single secondary user, cooperative spectrum sensing and its main elements and limiting factors have been discussed.

In Chapter 3, we first addressed the problem of sensing efficiency-accuracy trade-off through the design of a novel user-group assignment algorithm for group-based cooperative spectrum sensing based on energy detection. Our aim was to maximize the throughput efficiency that takes into account both the average opportunistic throughput and the average sensing overhead without degrading the sensing accuracy. We formulated the throughput efficiency maximization problem with heterogenous secondary users as a non-linear binary programming problem and designed the proposed assignment algorithm to solve it. The proposed algorithm adaptively performs user-group and group-sensing round assignments to minimize the number of sensing rounds and number of users needed to discover an available channel and thus increasing the throughput efficiency while satisfying a predefined limit on the sensing accuracy. We showed that the proposed algorithm has a low computational complexity and its performance is within $4.2 \%$ and $2.75 \%$ of the optimal exhaustive search for the fixed and variable secondary transmission rates cases, respectively, when the number of groups, $|G|=2$. We examined the effect of different parameters such as number of coop- 
erating users and channels, channel availability and different values of the signal-to-noise ratio on the proposed assignment algorithm. We showed that the proposed algorithm was consistently able to outperform the non-adaptive and sequential schemes for the different parameters examined. For example, for the variable transmission rate case, simulation results demonstrated that the proposed algorithm can provide improvement in the throughput efficiency for up to $19 \%$ and $86 \%$ when compared to non-adaptive and sequential cooperative sensing schemes, respectively, when the number of groups, $|G|=6$ and the number of secondary users, $K=12$.

In Chapter 4, we addressed the problem of reliably fusing the decisions of secondary users with heterogeneous sensing abilities. We proposed a decision fusion scheme that takes into consideration the reliability of each secondary user, in the form of a weight factor, when making the final decision at the fusion center. We defined the reliability of a secondary user's decision in terms of its probabilities of detection and false alarm. Contrary to most previous work, we did not assume the knowledge of the probabilities of detection and false alarm for each secondary user. Instead, we used the fusion center results and the local decisions made by secondary users to estimate the probabilities of detection and false alarm for each secondary user according to their relative frequencies through a counting process. We formulated the problem of minimizing the network probability of sensing error with heterogeneous secondary users subject to a limit on the network probability of detection as a non-linear integer programming problem. To solve the formulated optimization problem, we developed a dual search algorithm, based on a non-linear Lagrangian approach, and showed that the algorithm converges to the optimal value, obtained through exhaustive search, with zero duality gap using few number of iterations. We investigated through simulations the effect of different parameters such as the window size, global threshold and SNR on the proposed reliability-based fusion scheme. We showed that after a certain window size, that is different for different values of the global threshold and SNR, we can achieve a good estimate of the network probabilities of detection, false alarm and sensing error. We also 
showed that the proposed scheme is able to provide lower network probability of sensing error when compared to the OR and AND fusion schemes. For example, the proposed scheme can provide improvement in the network probability of sensing error for up to $18 \%$ and $88 \%$ when compared to the OR and AND fusion rules, respectively, when the number of secondary users, $K=8$.

In Chapter 5, we addressed the practical concern of secondary users reporting correlated local decisions to the fusion center. We employed a correlation model that uses a single parameter to quantify the degree of correlation between the secondary users' local decisions. We derived the system probabilities of detection and false alarm for the general $K$-out-of- $M$ fusion rule and showed that the detection performance of the cooperative spectrum sensing scheme degrades with the increase in the correlation between secondary users' local decisions. We formulated the problem of minimizing the network probability of sensing error as a mixed integer non-linear programming problem and showed that the number of secondary users that minimizes the network probability of sensing error is different for different values of the correlation parameter. Based on our results, we employed the genetic algorithm to find the optimal $K^{*}$-out-of- $M$ fusion rule and the optimal local threshold that jointly minimize the network probability of sensing error for cooperative spectrum sensing when the correlation index is known at the fusion center.

In practical scenarios, there may exist sensing errors due to time offset between local sensing observations performed by cooperating secondary users and the final sensing decision by the fusion center. This time offset can be caused by different factors such as reporting delay, synchronization issues or asynchronous reporting by secondary users. In Chapter 6, we analyzed the impact of sensing errors due to time offset on the performance of multiband cooperative joint detection. We investigated the performance improvement, in terms of aggregate opportunistic throughput, for the multiband joint detection framework when hard decision cooperation is employed at the fusion center. We employed a probability-based scheme for combining the local decisions of secondary users to take into account possible 
sensing errors at the fusion center due to time offset. We derived the opportunistic throughput and interference to primary users on each subchannel in the presence of time offset. We then formulated the problem of maximizing the aggregate opportunistic throughput for the cooperative multiband joint detection subject to a limit on the aggregate interference to primary users. We evaluated the performance of multiband cooperative joint detection in the presence of time offset and showed that as the time offset increases, the probability of false alarm on each subchannel also increases. This increase results in lower channel utilization and consequently leads to performance degradation in terms of the aggregate opportunistic throughput.

\subsection{Future Work}

Throughout this thesis, we proposed several techniques that contributed to the efficient design of cooperative spectrum sensing schemes for cognitive radio networks. However, there are some relevant issues that warrant further consideration in the future work. For instance, although using energy detection greatly reduces the complexity compared to other detection schemes, it relies on the assumption that the noise variance can be accurately estimated. However, in practice, this is difficult and the noise uncertainty will degrade the system performance even if cooperative spectrum sensing schemes are adopted. Therefore, building upon the work presented in this thesis, a two-stage sensing scheme can be employed wherein coarse sensing based on energy detection is performed in the first stage and, if required, fine sensing based on feature detection, such cyclostationary-based detection, can be performed in the second stage.

While studying the performance of the proposed cooperative sensing techniques, it has been assumed that a spectrum access opportunity for secondary users exists when the primary transmitter is inactive. However, secondary users can still share the spectrum when the primary user is transmitting provided that the amount of interference generated at the 
primary receiver is not harmful as described in Section 2.3. To protect the primary receiver, a guard area can be defined around each receiver where the secondary transmission is not permitted. The spectrum sensing problem can then be viewed as deciding whether or not the secondary transmitter is within the guard area. In the case where the secondary user can detect the primary user's transmitter but can still be allowed to transmit, the hypotheses may need to be modified in some reasonable way that accounts for those spatial spectrum opportunities. The probabilities of detection and false alarm will need to be computed using this modified formulation. The proposed cooperative spectrum sensing techniques in this work depend on the values of those probabilities and not on their specific distributions. This suggests that the proposed techniques can still be applied to improve the detection performance. However, further performance analysis and evaluations need to be carried out to assess this performance improvement.

In this work, we examined the performance of cooperative spectrum sensing over the additive white Gaussian noise channel. It will be beneficial to study the performance of the designed cooperative spectrum techniques over fading channels. When fading models, such as Rayleigh, Rician or Nakagami-m channels, are considered, the channel gain will be time variant and the SNR will be modelled as random variable with a distribution depending on the fading model used. Depending on the fading model, the average probability of detection can be evaluated by averaging the probability of detection obtained for the AWGN channel over the SNR range. Although we expect our designed techniques to provide performance improvement for cooperative spectrum sensing when fading is considered, this needs to be confirmed through analysis and simulations.

In this work, we considered the performance of cooperative spectrum sensing with correlated local decisions for identical secondary users. Assuming heterogeneous secondary users with correlated local decisions is an idea that warrants further investigation. The correlation model considered in this thesis can still be employed, however, the network probability of detection (false alarm) with unequal probabilities will need to be derived in the presence of 
correlation. Another interesting idea for future work is to incorporate the effect of correlation in the design of the user-group assignment algorithm proposed in Chapter 3. We expect that selecting users with minimum correlation to sense in each group can further enhance the performance of the proposed group-based cooperative spectrum sensing scheme. However, further research work is needed to come up with solid conclusions.

The analysis of the time offset impact on the performance of the multiband cooperative joint detection framework presented in Chapter 6 was the first step towards the design of a more robust fusion scheme for multiband cooperative sensing. Assuming different time offsets for different cooperating users can be easily incorporated in our analysis. This topic can be further investigated and extended to consider mitigating the effect of sensing errors due to time offset as a future research direction. For instance, the interference and spectrum utilization constraints need to be adjusted to account for the effect of those sensing errors. Since this will further increase the complexity of the optimization problem, efficient heuristics with reduced complexity will need to be developed. 


\section{Appendix A}

\section{Some Concepts in Optimization}

The goal of optimization is to find the best solution to a problem from a set of feasible solutions, such that a given quantity is optimized. The solutions are compared using a realvalued objective function of one or more problem variables. The feasible set is determined by the constraints, a system of inequalities or equations in the problem variables.

\section{A.1 Classification of Optimization Problems}

Optimization problems can be classified based on a number of characteristics as follows [147]:

- Number of variables: A problem with only one variable to be optimized is referred to as a univariate problem. If more than one variable is considered, the problem is referred to as a multivariate problem.

- Type of variables: A continuous problem has continuous-valued variables. The problem is referred to as an integer or discrete optimization problem if the variables are restricted to take integer values. A mixed integer problem has both continuousvalued and integer-valued variables. Problems where solutions are permutations of integer-valued variables are referred to as combinatorial optimization problems. 
- The number of optima: If there exists only one clear solution, the problem is unimodal. If more than one optimum exists, the problem is multimodal.

- Degree of non-linearity of the objective function: Linear problems have an objective function that is linear in the variables. Quadratic problems use quadratic functions. When other non-linear objective functions are used, the problem is referred to as a non-linear problem.

- The constraints used: A problem that uses only boundary constraints is referred to as an unconstrained problem. Constrained problems have additional equality and/or inequality constraints.

- The number of optimization criteria: If the quantity to be optimized is expressed using only one objective function, the problem is referred to as single objective problem. A multi-objective problem specifies more than one objective, which need to be simultaneously optimized.

In this thesis, we dealt with different classes of non-linear integer programming (NLIP) problems. NLIP problems are optimization problems where the set of variables are constrained to take integer values and the objective function and feasible region of the problem are described by non-linear functions (optimization problem in Chapter 4). If those variables are restricted to binary values (0 or 1$)$, the problem is called non-linear binary (0-1) programming problem (optimization problem in Chapter 3). If some variables are integers and some can take continuous values, the problem becomes a mixed integer non-linear (MINLP) programming problem (optimization problem in Chapter 5).

\section{A.2 Classes of Complexity}

Classes of complexity are defined to distinguish problems according to their hardness. The complexity class $P$, which stands for polynomial, consists of all those problems that can 
be solved on a deterministic Turing machine in polynomial time from the size of the input. Turing machines are an abstraction that is used to formalize the notion of algorithm and computational complexity. A comprehensive description of them can be found in [148]. On the other hand, the complexity class NP, which stands for non-deterministic polynomial, consists of all those problems whose solution can be found in polynomial time on a nondeterministic Turing machine. Since such a machine does not exist, practically it means that an exponential algorithm can be written for an NP-problem, nothing is asserted whether a polynomial algorithm exists or not. Problems in the $N P$ complexity class have the following properties:

- They are decision problems.

- They can be solved in polynomial time on a non-deterministic Turing machine.

- Their solution can be verified for correctness in polynomial time on a deterministic Turing machine.

A subclass of $N P$ is called $N P$-complete. Any $N P$ problem lies in the class $N P$-complete if and only if every other problem in $N P$ can be transformed to it in polynomial time. Informally, NP-complete problems are the hardest problems in NP. Moreover, if anyone could find a polynomial algorithm to solve any $N P$-complete problem on a deterministic Turing machine, this implies that every problem in class $N P$ can be solved in polynomial time. However, no such algorithm has been found yet.

Finally, the class NP-hard can be understood as the set of problems that are at least as hard as the hardest problems in $N P$. In other words, $N P$-hard problems have the same trait as $N P$-complete problems but they do not necessary belong to class $N P$. Based on this definition, if an optimization problem has an NP-complete decision version, then this optimization problem is NP-hard. Since the decision version of the linear integer binary problem is in NP-complete [148], the linear integer programming optimization problem is $N P$-hard. Since any bounded integer variable can be expressed as a combination of binary 
variables, both the linear integer and mixed integer linear optimization problems are generally NP-hard. Therefore, the considered classes of non-linear integer optimization problems are also NP-hard since they generalize their equivalent classes of linear integer optimization problems. For optimization problems that belong to class $N P$-hard, it is unlikely to find an optimal solution within a practical amount of time. In such cases, heuristic algorithms that find suboptimal solutions, but have acceptable time and space (memory) complexity, play an indispensable role.

\section{A.3 Computational Complexity of Algorithms}

A major characteristic of a good algorithm is its efficiency, that is, how much time and memory are required to solve a particular problem. Intuitively, time and memory can be measured in real units such as seconds and megabytes. However, these measurements are not subjective for comparisons between algorithms, because they depend on the computing power of the specific machine and on the specific data set. To standardize the measurement of algorithm efficiency, the computational complexity theory was developed [149]. This allows the efficiency of an algorithm to be estimated and expressed conceptually as a mathematical function of its input size, $m$, which refers to the number of items in the input data set.

In general, time complexity is considered more important than space complexity, in part because the memory requirement of most algorithms is lower than the capacity of current machines. The time complexity of an algorithm can be measured using best-case, averagecase or worst-case complexity. The best-case complexity, which is less commonly used, is when an algorithm takes minimum amount of time to run to completion for a specific set

of inputs. Average-case complexity is a more meaningful characterization of an algorithm, however, its calculations are often difficult and complicated. This necessitates the use of a worst-case complexity metric which represents the complexity of an algorithm with respect to the worst possible inputs. Therefore, worst-case complexity gives an upper bound on the 
average-case complexity.

Table A.1: Table of Common Time Complexities as a Function of the Input Size $m$.

\begin{tabular}{|c|c|}
\hline Notation & Name \\
\hline$O(1)$ & Constant time \\
\hline$O(\log m)$ & Logarithmic time \\
\hline$O(m)$ & Linear time \\
\hline$O(m \log m)$ & Log-linear time \\
\hline$O\left(m^{2}\right)$ & Quadratic time \\
\hline$O\left(m^{3}\right)$ & Cubic time \\
\hline$O\left(2^{m}\right)$ & Exponential time \\
\hline$O(m !)$ & Factorial time \\
\hline
\end{tabular}

In computational complexity theory, not all parts of the running time of an algorithm are essential. In fact, only the rate of growth or the order of growth of the running time is typically of most concern in comparing the complexities of different algorithms. Asymptotic notations are symbols used in computational complexity theory to express the efficiency of algorithms with a focus on their orders of growth. The three most used notations are $O$-notation, $\Omega$-notation, and $\Theta$-notation, which are explained as follows [150]:

- O-notation: The $O$-notation, which denotes the asymptotic upper bounds of the complexity functions, is the dominant method used to express the complexity of algorithms. In $O$-notation, lower-order terms and constant coefficients of leading terms can be ignored in complexity theory. If the time complexity of an algorithm can be expressed with or is asymptotically bounded by a polynomial function, it has polynomial time complexity. Otherwise, it has exponential time complexity. The $O$-notations of frequently encountered order of functions are listed in Table A.1 in descending order of their efficiency.

- $\Omega$-notation: The $\Omega$-notation is the inverse of $O$-notation and is used to express the asymptotic lower bounds of complexity functions. $\Omega$-notation receives much less attention than $O$-notation, because we are usually concerned about how much time at most would be spent executing an algorithm instead of the least amount of time spent. 
- $\Theta$-notation: The $\Theta$-notation expresses the asymptotically tight bounds of complexity functions. Although $\Theta$-notation is more precise than $O$-notation when characterizing algorithm complexity, $O$-notation is usually favored since it is often much easier to prove an upper bound than it is to prove a tight bound. Moreover, upper bounds are considered sufficient for characterizing algorithm complexity. 


\section{Appendix B}

\section{List of Publications}

\section{B.1 Journal Publications}

[1] L. Khalid and A. Anpalagan, "Adaptive Assignment of Heterogeneous Users for GroupBased Cooperative Spectrum Sensing," Under Revision, 2013.

[2] L. Khalid and A. Anpalagan, "Adaptive Decision Fusion Scheme for Cooperative Spectrum Sensing in Cognitive Radio Networks," Under Revision, 2013.

[3] L. Khalid and A. Anpalagan, "Cooperative Sensing with Correlated Local Decisions in Cognitive Radio Networks," IEEE Transactions on Vehicular Technology, vol. 61, pp. 843-849, February 2012.

[4] L. Khalid and A. Anpalagan, "Emerging Cognitive Radio Technology: Principles, Challenges and Opportunities," Computers and Electrical Engineering, vol. 2, pp. 358-366, March 2010.

\section{B.2 Conference Publications}

[1] L. Khalid and A. Anpalagan, "Adaptive Grouping Scheme for Cooperative Spectrum Sensing in Cognitive Radio Networks," Submitted to IEEE Vehicular Technology Con- 
ference, 2014.

[2] L. Khalid and A. Anpalagan, "Performance of Cooperative Spectrum Sensing with Correlated Cognitive Users Decisions," in IEEE International Symposium on Personal, Indoor and Mobile Radio Communications, (Toronto, Canada), pp. 635-639, September 2011.

[3] L. Khalid and A. Anpalagan, "A Weighted Fusion Scheme for Cooperative Spectrum Sensing based on Past Decisions," in IEEE International Symposium on Personal, Indoor and Mobile Radio Communications, (Toronto, Canada), pp. 354-358, September 2011.

[4] L. Khalid and A. Anpalagan, "Effect of Sensing Errors on Wideband Cognitive OFDM Radio Networks," in IEEE Queens Biennial Symposium on Communications, (Kingston, Canada), pp. 273-277, May 2010.

[5] L. Khalid, K. Raahemifar, and A. Anpalagan, "Cooperative Spectrum Sensing for Wideband Cognitive OFDM Radio Networks," in IEEE Vehicular Technology Conference, (Anchorage, AK), pp. 1-5, September 2009. 


\section{Bibliography}

[1] Federal Communications Commission, "Spectrum Policy Task Force Report," ET Docket no. 02-135, November 2002.

[2] I. Mitola and J. Maguire, "Cognitive Radio: Making Software Radios More Personal," IEEE Personal Communications Magazine, vol. 6, no. 4, pp. 13-18, August 1999.

[3] Y. Liang, K. Chen, G. Y. Li, and P. Mhnen, "Cognitive Radio Networking and Communications: An Overview," IEEE Transactions On Vehicular Technology, vol. 60, no. 7, pp. 3386-3407, September 2011.

[4] Federal Communications Commission, "Notice of Proposed Rulemaking, in the Matter of Unlicensed Operation in the TV Broadcast Bands (ET Docket no. 04-186) and Additional Spectrum for Unlicensed Devices below $900 \mathrm{MHz}$ and in the $3 \mathrm{GHz}$ Band (ET Docket no. 02-380)," Tech. Rep. FCC 04-113, May 2004.

[5] M. Nekovee, "Cognitive Radio Access to TV White Spaces: Spectrum Opportunities, Commercial Applications and Remaining Technology Challenges," in IEEE International Symposium on New Frontiers in Dynamic Spectrum Access Networks, Singapore, April 2010, pp. 1-10.

[6] A. Ghasemi and E. S. Sousa, "Spectrum Sensing in Cognitive Radio Networks: Requirements, Challenges and Design Trade-offs," IEEE Communications Magazine, vol. 46, no. 4, pp. 32-39, April 2008. 
[7] C. R. Stevenson, G. Chouinard, Z. Lei, W. Hu, S. J. Shellhammer, and W. Caldwell, "IEEE 802.22: The First Cognitive Radio Wireless Regional Area Network Standard," IEEE Communications Magazine, vol. 47, no. 1, pp. 130-138, January 2009.

[8] S. Haykin, "Cognitive Radio: Brain-Empowered Wireless Communications," IEEE Journal on Selected Areas in Communications, vol. 23, no. 2, pp. 201-220, Febraury 2005.

[9] I. F. Akyildiz, W. Lee, M. C. Vuran, and S. Mohanty, "Next Generation/Dynamic Spectrum Access/Cognitive Radio Wireless Networks: A Survey," The International Journal of Computer and Telecommunications Networking, vol. 50, no. 13, pp. 21272159, September 2006.

[10] A. Goldsmith, S. A. Jafar, I. Maric, and S. Srinivasa, "Breaking Spectrum Gridlock with Cognitive Radios: An Information Theoretic Perspective ," Proceedings of the IEEE, vol. 97, no. 5, pp. 894-914, May 2009.

[11] W. Webb, "On using White Space Spectrum," IEEE Communications Magazine, vol. 50, no. 8, pp. 145-151, August 2012.

[12] T. Yucek and H. Arslan, "A Survey of Spectrum Sensing Algorithms for Cognitive Radio Applications," IEEE Communications Surveys and Tutorials, vol. 11, no. 1, pp. 116-130, March 2009.

[13] S. Haykin, D. Thomson, and J. Reed, "Spectrum Sensing for Cognitive Radio," Proceedings of the IEEE, vol. 97, no. 5, pp. 849-877, May 2010.

[14] D. Cabric, S. Mishra, and R. Brodersen, "Implementation Issues in Spectrum Sensing for Cognitive Radio," in Asilomar Conference on Signals, Systems and Computers, vol. 1, Pacific Grove, CA, November 2004, pp. 772-776. 
[15] A. Ghasemi and E. Sousa, "Optimization of Spectrum Sensing for Opportunistic Spectrum Access in Cognitive Radio Networks," in IEEE Consumer Communications and Networking Conference, Las Vegas, NV, January 2007, pp. 1022-1026.

[16] J. Unnikrishnan and V. Veeravalli, "Cooperative Spectrum Sensing and Detection for Cognitive Radio," in IEEE Global Telecommunications Conference, Washington, DC, November 2007, pp. 2972-2976.

[17] J. Unnikrishnan and V. V. Veeravalli, "Cooperative Sensing for Primary Detection in Cognitive Radio," IEEE Journal of Selected Topics in Signal Processing, vol. 2, no. 1, pp. 18-27, February 2008.

[18] G. Ganesan and Y. Li, "Cooperative Spectrum Sensing in Cognitive Radio Networks," in IEEE International Symposium on New Frontiers in Dynamic Spectrum Access Networks, Baltimore, MD, November 2005, pp. 137-143.

[19] I. F. Akyildiz, B. F. Lo, and R. Balakrishnan, "Cooperative Spectrum Sensing in Cognitive Radio Networks: A Survey," Physical Communication (Elsevier) Journal, vol. 4, no. 1, pp. 40-62, March 2011.

[20] S. Mishra, A. Sahai, and R. Brodersen, "Cooperative Sensing Among Cognitive Radios," in IEEE International Conference on Communications, vol. 4, Istanbul, Turkey, June 2006, pp. 1658-1663.

[21] S. Huang, X. Liu, and Z. Ding, "Optimal Sensing-Transmission Structure for Dynamic Spectrum Access," in IEEE Conference on Computer Communications, Rio de Janeiro, Brazil, April 2009, pp. 2295-2303.

[22] L. Khalid and A. Anpalagan, "Emerging Cognitive Radio Technology: Principles, Challenges and Opportunities," Computers and Electrical Engineering (Elsevier) Journal, vol. 2, no. 36, pp. 358-366, March 2010. 
[23] A. Sahai, N. Hoven, and R. Tandra, "Some Fundamental Limits on Cognitive Radio," in Allerton Conference on Communication, Control and Computing, Monticello, IL, October 2004.

[24] F. Digham, M. Alouini, and M. Simon, "On the Energy Detection of Unknown Signals over Fading Channels," in IEEE International Conference on Communications, vol. 5, Anchorage, AK, May 2003, pp. 3575-3579.

[25] E. Vistotsky, S. Kuffner, and R. Peterson, "On Collaborative Detection of TV Transmissions in Support of Dynamic Spectrum Sharing," in IEEE International Symposium on New Frontiers in Dynamic Spectrum Access Networks, Baltimore, MD, November 2005, pp. 338-345.

[26] J. Hillenbrand, T. A. Weiss, and F. K. Jondral, "Calculation of Detection and False Alarm Probabilities in Spectrum Pooling Systems," IEEE Communications Letters, vol. 9, no. 4, pp. 349-351, April 2005.

[27] Q. Liu, J. Gao, and L. Chen, "Optimization of Energy Detection Based Cooperative Spectrum Sensing in Cognitive Radio Networks," in IEEE International Conference on Wireless Communications and Signal Processing, Suzhou, China, October 2010, pp. $1-5$.

[28] E. Axel, G. Leus, E. Larsson, and H. V. Poor, "Spectrum Sensing for Cognitive Radio: State-of-the-Art and Recent Advances," IEEE Signal Processing Magazine, vol. 29, no. 3, pp. 101-116, May 2012.

[29] K. Koufos, "Spectrum Access in White Spaces using Spectrum Sensing and Geolocation Databases," Ph.D. dissertation, Aalto University, 2013.

[30] E. Hossain, D. Niyato, and Z. Han, Dynamic Spectrum Access and Management in Cognitive Radio Networks. Cambridge University Press, 2009. 
[31] A. G. Varnamkhasti, "Spectrum Sensing in Cognitive Wireless Networks: Requirements, Challenges annd Design Trade-offs," Ph.D. dissertation, University of Toronto, 2008.

[32] Y. Zeng, Y. Liang, A. T. Hoang, and R. Zhang, "A Review on Spectrum Sensing Techniques for Cognitive Radio: Challenges and Solutions," EURASIP Journal on Advances in Signal Processing, vol. 2010, no. 2, pp. 1-15, January 2010.

[33] R. Tandra and A. Sahai, "Some Fundamental Limits on Detection in Low SNR under Noise Uncertainty," in IEEE International Conference on Wireless Networks, Communications and Mobile Computing, vol. 1, Wuhan, China, June 2005, pp. 464-469.

[34] H. Arslan, Cognitive Radio, Software Defined Radio, and Adaptive Wireless Systems. Springer Netherlands, 2007.

[35] H.Urkowitz, "Energy Detection of Unknown Deterministic Signal," Proceedings of the IEEE, vol. 55, no. 4, pp. 523-531, April 1967.

[36] A. Ghasemi and E. Sousa, "Collaborative Spectrum Sensing for Opportunistic Access in Fading Environment," in IEEE International Symposium on New Frontiers in Dynamic Spectrum Access Networks, Baltimore, MD, November 2005, pp. 131-136.

[37] Z. Quan, S. Cui, and A. Sayed, "Optimal Linear Cooperation for Spectrum Sensing in Cognitive Radio Networks," IEEE Journal of Selected Topics in Signal Processing, vol. 2, no. 1, pp. 28-40, February 2008.

[38] Z. Quan, S. Cui, A. Sayed, and H. Poor, "Wideband Spectrum Sensing in Cognitive Radio Networks," in IEEE International Conference on Communications, Beijing, China, May 2008, pp. 901-906. 
[39] H. Tang, "Some Physical Layer Issues of Wide-band Cognitive Radio Systems," in IEEE International Symposium on New Frontiers in Dynamic Spectrum Access Networks, Baltimore, MD, November 2005, pp. 151-159.

[40] Z. Quan, S. Cui, A. Sayed, and H. Poor, "Optimal Multiband Joint Detection for Spectrum Sensing in Cognitive Radio Networks," IEEE Transactions on Signal Processing, vol. 57, no. 3, pp. 1128-1140, March 2009.

[41] D. Cabric and R. Brodersen, "Physical layer Design Issues Unique to Cognitive Radio Systems," in IEEE Personal Indoor and Mobile Radio Communications, vol. 2, Berlin, Germany, September 2005, pp. 759-763.

[42] Z. Tian and G. B. Giannakis, "A Wavelet Approach to Wideband Spectrum Sensing for Cognitive Radios," in IEEE International Conference on Cognitive Radio Oriented Wireless Networks and Communications, Mykonos Island, Greece, June 2006, pp. 151159.

[43] Z. Tian and G. Giannakis, "Compressed Sensing for Wideband Cognitive Radios," in IEEE International Conference on Acoustics, Speech, and Signal Processing, Honolulu, HI, April 2007, pp. 1357-1360.

[44] G. Ganesan and Y. Li, "Cooperative Spectrum Sensing in Cognitive Radio: Part I: Two User Networks," IEEE Transactions on Wireless Communications, vol. 6, no. 6, pp. 2204-2213, June 2007.

[45] N. S. Shankar, C. Cordeiro, and K. Challapali, "Spectrum Agile Radios: Utilization and Sensing Architectures," in IEEE International Symposium on New Frontiers in Dynamic Spectrum Access Networks, Baltimore, MD, November 2005, pp. 160-169.

[46] G. Ganesan and Y. Li, "Cooperative Spectrum Sensing in Cognitive Radio: Part II: Multiuser Networks," IEEE Transactions on Wireless Communications, vol. 6, no. 6, pp. 2214-2222, June 2007. 
[47] H. Uchiyama, K. Umebayashi, T. Fujii, F. Ono, K. Sakaguchi, Y. Kamiya, and Y. Suzuki, "Study on Soft Decision Based Cooperative Sensing for Cognitive Radio Networks," IEICE Transactions on Communications, vol. E91-B, no. 1, pp. 95-101, January 2008.

[48] J. Ma, G. Zhao, and Y. Li, "Soft Combination and Detection for Cooperative Spectrum Sensing in Cognitive Radio Networks," IEEE Transactions on Wireless Communications, vol. 7, no. 11, pp. 4502-4507, November 2008.

[49] E. Peh and Y. Liang, "Optimization for Cooperative Sensing in Cognitive Radio Networks," in IEEE Wireless Communications and Networking Conference, Hong Kong, China, March 2007, pp. 27-32.

[50] E. Hossain and V. Bhargava, Cognitive Wireless Communication Networks. Springer, 2007.

[51] Y. Liang, Y. Zeng, E. C. Peh, and A. T. Hoang, "Sensing-Throughput Tradeoff for Cognitive Radio Networks," IEEE Transactions on Wireless Communications, vol. 7, no. 4, pp. 1326-1337, April 2008.

[52] Z. Khan, J. Lehtomaki, K. Umebayashi, and J. Vartiainen, "On the Selection of the Best Detection Performance Sensors for Cognitive Radio Networks," IEEE Signal Processing Letter, vol. 17, no. 4, pp. 359-362, April 2010.

[53] W. Zhang, R. K. Mallik, and K. B. Letaief, "Cooperative Spectrum Sensing Optimization in Cognitive Radio Networks," in IEEE International Conference on Communications, Beijing, China, May 2008, pp. 3411-3415.

[54] X. Li, W. Li, and Y. Hei, "Joint Spectrum Sensing and User Selection Strategy for Cognitive Radio Networks," in IEEE International Conference on Wireless Communications and Signal Processing, Huangshan, China, October 2012, pp. 1-6. 
[55] A. S. Cacciapuoti, I. F. Akyildiz, and L. Paura, "Correlation-Aware User Selection for Cooperative Spectrum Sensing in Cognitive Radio Ad Hoc Networks," IEEE Journal on Selected Areas in Communications, vol. 30, no. 2, pp. 297-306, February 2012.

[56] K. Zeng, J. Wang, S. Li, and D. Cabric, "Robust Node Selection for Cooperative Spectrum Sensing with Malicious Users," in IEEE Military Communications Conference, Baltimore, MD, November 2011, pp. 79-84.

[57] W. Wang, H. Li, Y. Sun, and Z. Han, "Securing Collaborative Spectrum Sensing against Untrustworthy Secondary Users in Cognitive Radio Networks," EURASIP Journal on Advances in Signal Processing, vol. 2010, no. 4, pp. 106-117, January 2010.

[58] W. Y. Lee and I. F. Akyildiz, "Optimal Spectrum Sensing Framework for Cognitive Radio Networks," IEEE Transactions on Wireless Communications, vol. 7, no. 10, pp. 3845-3857, October 2008.

[59] R. Yu, Y. Zhang, L. Yi, S. Xie, L. Song, and M. Guizani, "Secondary Users Cooperation in Cognitive Radio Networks: Balancing Sensing Accuracy and Efficiency," IEEE Wireless Communications, vol. 19, no. 2, pp. 30-37, April 2012.

[60] H. Kim and K. G. Shin, "Efficient Discovery of Spectrum Opportunities with MACLayer Sensing in Cognitive Radio Networks," IEEE Transactions on Mobile Computing, vol. 7, no. 5, pp. 533-545, May 2008.

[61] S. Xie, Y. Liu, Y. Zhang, and R. Yu, "A Parallel Cooperative Spectrum Sensing in Cognitive Radio Networks," IEEE Transactions On Vehicular Technology, vol. 59, no. 8, pp. 4079-4092, October 2010.

[62] Y. Liu, R. Yu, Y. Zhang, and C. Yuen, "An Efficient MAC Protocol with Selective Grouping and Cooperative Sensing in Cognitive Radio Networks," IEEE Transactions On Vehicular Technology, vol. 62, no. 8, pp. 3928-3941, October 2013. 
[63] C. Sun, W. Zhang, and K. Letaief, "Cooperative Spectrum Sensing for Cognitive Radios under Bandwidth Constraints," in IEEE Wireless Communications and Networking Conference, Hong Kong, China, March 2007, pp. 1-5.

[64] H. Pham, Y. Zhang, P. Engelstad, T. Skeie, and F. Eliassen, "Energy Minimization Approach for Optimal Cooperative Spectrum Sensing in Sensor-Aided Cognitive Radio Networks," in International ICST Conference on Wireless Internet, Singapore, March 2010, pp. 1-9.

[65] D. C. Oh and Y. H. Lee, "Energy Detection Based Spectrum Sensing for Sensing Error Minimization in Cognitive Radio Networks," International Journal of Communication Networks and Information Security, vol. 1, no. 1, pp. 1-5, April 2009.

[66] T. Jiang and D. Qu, "On Minimum Sensing Error with Spectrum Sensing using Counting Rule in Cognitive Radio Networks," in International ICST Conference on Wireless Internet, Maui, HI, November 2008, pp. 1-9.

[67] L. Khalid and A. Anpalagan, "Cooperative Sensing with Correlated Local Decisions in Cognitive Radio Networks," IEEE Transactions on Vehicular Technology, vol. 61, no. 2, pp. 843-849, February 2012.

[68] X. Zhou, J. Ma, G. Y. Li, Y. H. Kwon, and A. C. Soong, "Probability-Based Combination for Cooperative Spectrum Sensing," IEEE Transactions on Communications, vol. 58, no. 2, pp. 463-466, February 2010.

[69] Y. Chen and N. Beaulieu, "Performance of Collaborative Spectrum Sensing for Cognitive Radio in the Presence of Gaussian Channel Estimation Errors," IEEE Transactions on Communications, vol. 57, no. 7, pp. 1944-1947, July 2009.

[70] D. Donoho, "Compressed Sensing," IEEE Transactions on Information Theory, vol. 52, no. 4, pp. 1289-1306, April 2006. 
[71] T. Ragheb, S. Kirolos, J. Laska, A. Gilbert, M. Strauss, R. Baraniuk, and Y. Massoud, "Implementation Models for Analog-to-Information Conversion via Random Sampling," in Midwest Symposium on Circuits and Systems, Montreal, Canada, August 2007, pp. 325-328.

[72] P. Paysarvi-Hoseini and N. C. Beaulieu, "Optimal Wideband Spectrum Sensing Framework for Cognitive Radio Systems," IEEE Transactions on Signal Processing, vol. 59, no. 3, pp. 1170-1182, March 2011.

[73] Y. Liu, R. Yu, Y. Zhang, and S. Xie, "A Group-based Cooperative Medium Access Control Protocol for Cognitive Radio Networks," in IEEE International Workshop On Quality Of Service, Beijing, China, June 2010, pp. 1-9.

[74] S. Atapattu, C. Tellambura, and H. Jiang, "Energy Detection Based Cooperative Spectrum Sensing in Cognitive Radio Networks," IEEE Transactions on Wireless Communnications, vol. 10, no. 4, pp. 1232-1241, April 2011.

[75] R. Deng, J. Chen, C. Yuen, P. Cheng, and Y. Sun, "Energy-Efficient Cooperative Spectrum Sensing by Optimal Scheduling in Sensor-Aided Cognitive Radio Networks," IEEE Transactions on Vehicular Technology, vol. 61, no. 2, pp. 716-725, February 2012.

[76] Y. Chen, "Optimum Number of Secondary Users in Collaborative Spectrum Sensing Considering Resources Usage Efficiency," IEEE Communications Letters, vol. 12, no. 11, pp. 877-879, December 2008.

[77] J. Shen, S. Liu, L. Zeng, G. Xie, J. Gao, and Y. Liu, "Optimisation of Cooperative Spectrum Sensing in Cognitive Radio Network," IET Communications, vol. 3, no. 7, pp. 1170-1178, July 2009. 
[78] R. Fan and H. Jiang, "Optimal Multi-Channel Cooperative Sensing in Cognitive Radio Networks," IEEE Transactions on Wireless Communications, vol. 9, no. 3, pp. 11281138, March 2010.

[79] H. Su and X. Zhang, "Cross-Layer Based Opportunistic MAC Protocols for QoS Provisionings Over Cognitive Radio Wireless Networks," IEEE Journal On Selected Areas In Communications, vol. 26, no. 1, pp. 118-129, January 2008.

[80] Y. Liu, R. Yu, and S. Xie, "Optimal Cooperative Sensing Scheme under Time-Varying Channel for Cognitive Radio Networks," in IEEE International Symposium on New Frontiers in Dynamic Spectrum Access Networks, Chicago, IL, November 2008, pp. $352-369$.

[81] E. Peh, Y. Liang, Y. L. Guan, and Y. Zeng, "Optimization of Cooperative Sensing in Cognitive Radio Networks: A Sensing-Throughput Tradeoff View," IEEE Transactions On Vehicular Technology, vol. 58, no. 9, pp. 5294-5299, November 2009.

[82] B. V. Gendenko and A. N. Kolmogorov, Limit Distributions for Sums of Independent Random Variables. Addison-Wesley, 1954.

[83] B. Vujitic, N. Cackov, S. Vujicic, and L. Trajkovid, "Modeling and Characterization of Traffic in Public Safety Wireless Networks," in International Symposium on Performance Evaluation of Computer and Telecommunication Systems, Philadelphia, PA, July 2005, pp. 212-233.

[84] Y. Wu, B. Wang, and K. R. Liu, "Optimal Defense Against Jamming Attacks in Cognitive Radio Networks using the Markov Decision Process Approach," in IEEE Global Telecommunications Conference, Miami, FL, December 2010, pp. 1-5.

[85] R. Bacchus, T. Taher, K. Zdunek, and D. Roberson, "Spectrum Utilization Study in Support of Dynamic Spectrum Access for Public Safety," in IEEE International 
Symposium on New Frontiers in Dynamic Spectrum Access Networks, Singapore, April 2010, pp. 1-11.

[86] S. Geirhofer, L. Tong, and B. M. Sadler, "Dynamic Spectrum Access in the Time Domain: Modeling and Exploiting White Space," IEEE Communications Magazine, vol. 45, no. 5, pp. 66-72, May 2007.

[87] H. T. Cheng and W. Zhuang, "Simple Channel Sensing Order in Cognitive Radio Networks," IEEE Journal On Selected Areas In Communications, vol. 29, no. 4, pp. 676-688, April 2011.

[88] M. B. Ghorbel, H. Nam, and M. Alouini, "Cluster-Based Spectrum Sensing for Cognitive Radios with Imperfect Channel to Cluster-Head," in IEEE Wireless Communications and Networking Conference, Paris, France, April 2012, pp. 709-713.

[89] H. Jiang, L. Lai, R. Fan, and H. V. Poor, "Optimal Selection of Channel Sensing Order in Cognitive Radio," IEEE Transactions On Wireless Communications, vol. 8, no. 1, pp. 297-307, January 2009.

[90] R. Fan and H. Jiang, "Channel Sensing-Order Setting in Cognitive Radio Networks: A Two-User Case," IEEE Transactions On Vehicular Technology, vol. 58, no. 9, pp. 4997-5008, November 2009.

[91] R. Hemmecke, M. Koppe, J. Lee, and R. Weismantel, 50 Years of Integer Programming 1958-2008. Springer, 2010, ch. Nonlinear Integer Programming, pp. 561-618.

[92] H. Kim and K. G. Shin, "In-Band Spectrum Sensing in IEEE 802.22 WRANs for Incumbent Protection," IEEE Transactions on Mobile Computing, vol. 9, no. 12, pp. 1766-1779, December 2010. 
[93] C. Cordeiro, K. Challapali, D. Birru, and S. Shankar, "IEEE 802.22: An Introduction to the First Wireless Standard based on Cognitive Radios," Journal of Communications, vol. 1, no. 1, pp. 38-47, April 2006.

[94] Y. Xu and D. Li, "A Non-linear Lagrangian Dual for Integer Programming," Operations Research Letter, vol. 30, no. 6, pp. 401-407, December 2002.

[95] E. C. Peh, Y. Liang, Y. L. Guan, and Y. Zeng, "Cooperative Spectrum Sensing in Cognitive Radio Networks with Weighted Decision Fusion Schemes," IEEE Transactions on Wireless Communications, vol. 9, no. 12, pp. 3838-3847, December 2010.

[96] P. Qihang, Z. Kun, W. Jun, and L. Shaoqian, "A Distributed Spectrum Sensing Scheme Based on Credibility and Evidence Theory in Cognitive Radio Context," in IEEE International Symposium on Personal, Indoor and Mobile Radio Communications, Helsinki, Finland, September 2006, pp. 1-5.

[97] N. Nguyen-Thanh and I. Koo, "An Enhanced Cooperative Spectrum Sensing Scheme Based on Evidence Theory and Reliability Source Evaluation in Cognitive Radio Context," IEEE Communications Letters, vol. 13, no. 7, pp. 492-494, July 2009.

[98] T. Qin, H. Yu, C. Leung, Z. Shen, and C. Miao, "Towards a Trust Aware Cognitive Radio Architecture," ACM SIGMOBILE Mobile Computing and Communications Review, vol. 13, no. 2, pp. 86-95, April 2009.

[99] J. Feng, G. Lu, and Z. Bao, "Weighted-Cooperative Spectrum Sensing Scheme using Trust in Cognitive Radio Networks," in IEEE International Conference on Signal Processing, Beijing, China, October 2012, pp. 1693-1696.

[100] D. C. Oh, H. C. Lee, and Y. H. Lee, "Linear Hard Decision Combining for Cooperative Spectrum Sensing in Cognitive Radio Systems," in IEEE Vehicular Technology Conference, Ottawa, Canada, September 2010, pp. 1-5. 
[101] B. Shen, L. Huang, C. Zhao, K. Kwak, and Z. Zhou, "Weighted Cooperative Spectrum Sensing in Cognitive Radio Networks," in International Conference on Convergence and Hybrid Information Technology, vol. 1, Busan, Korea, November 2008, pp. 10741079.

[102] M. B. Shahid and J. Kamruzzaman, "Weighted Soft Decision for Cooperative Sensing in Cognitive Radio Networks ," in IEEE International Conference on Networks, New Delhi, India, December 2008, pp. 1-6.

[103] Y. Zhao, M. Song, and C. Xin, "A Weighted Cooperative Spectrum Sensing Framework for Infrastructure-Based Cognitive Radio Networks," Computer Communications, vol. 34, no. 12, pp. 1510-1517, August 2011.

[104] N. Hasan, W. Ejaz, and H. S. Kim, "PWAM: Penalty-Based Weighted Adjustment Mechanism for Cooperative Spectrum Sensing in Centralized Cognitive Radios Networks," International Journal of Innovative Computing, Information and Control, vol. 8, no. 10, pp. 1510-1517, October 2012.

[105] L. Chuan-qing and W. Zhi-ming, "Adaptive Weighted Algorithm Of Cooperative Spectrum Sensing in Cognitive Radio Networks," in IET International Communication Conference on Wireless Mobile and Computing, Shanghi, China, November 2011, pp. $121-126$.

[106] M. Zhou, H. Chen, L. Xie, and K. Wang, "A Reliable Collaborative Spectrum Sensing Scheme Based on the ROCQ Reputation Model for Cognitive Radio Networks," in IEEE Vehicular Technology Conference, Quebec City, Canada, May 2012, pp. 1-5.

[107] N. Ansari, E. S. H. Hou, B. Zhu, and J. Chen, "Adaptive Fusion by Reinforcement Learning for Distributed Detection Systems," IEEE Transactions on Aerospace and Electronic Systems, vol. 32, no. 2, pp. 524-531, April 1996. 
[108] N. Nguyen-Thanh and I. Koo, "A Secure Distributed Spectrum Sensing Scheme in Cognitive Radio," in Emerging Intelligent Computing Technology and Applications With Aspects of Artificial Intelligence, ser. Lecture Notes in Computer Science. Springer Berlin / Heidelberg, 2009, vol. 5755, pp. 698-707.

[109] L. Khalid and A. Anpalagan, "A Weighted Fusion Scheme for Cooperative Spectrum Sensing based on Past Decisions," in IEEE International Symposium on Personal, Indoor and Mobile Radio Communications, Toronto, Canada, September 2011, pp. 354-358.

[110] A. L. Garcia, Probability, Statistics, and Random Processes for Electrical Engineering, 3rd ed. Prentice Hall, December 2007.

[111] D. Li and X. Sun, Nonlinear Integer Programming. Springer, 2006.

[112] Y. Xu, C. Liu, and D. Li, "Generalized Nonlinear Lagrangian Formulation for Bounded Integer Programming," Journal of Global Optimization, vol. 33, no. 2, pp. 257-272, October 2005.

[113] M. Abramson, C. Audet, G. Couture, J. D. Jr., and S. LeDigabel, "The NOMAD Project," http://www.gerad.ca/nomad/.

[114] C. Audet and J. E. D. Jr., "Mesh Adaptive Direct Search Algorithms for Constrained Optimization," SIAM Journal on Optimization, vol. 17, no. 1, pp. 188-217, 2006.

[115] L. M. Rios and N. V. Sahinidis, "Derivative-free Optimization: A Review of Algorithms and Comparison of Software Implementations," Journal of Global Optimization, vol. 56, no. 3, pp. 1247-1293, July 2013.

[116] M. D. Renzo, F. Graziosi, and F. Santucci, "Cooperative Spectrum Sensing in Cognitive Radio Networks over Correlated Log-Normal Shadowing," in IEEE Vehicular Technology Conference, Barcelona, Spain, April 2009, pp. 1-5. 
[117] A. Konaka, D. W. Coitb, and A. E. Smith, "Multi-objective Optimization using Genetic Algorithms: A Tutorial," Reliability Engineering and System Safety, vol. 91, no. 9, pp. 992-1007, September 2006.

[118] P. K. Varshney, Distributed Detection and Data Fusion. New York: Springer-Verlag, 1996.

[119] W. Zhang, R. K. Mallik, and K. Letaief, "Optimization of Cooperative Spectrum Sensing with Energy Detection in Cognitive Radio Networks," IEEE Transactions on Wireless Communications, vol. 8, no. 12, pp. 5761-5766, December 2009.

[120] X. Liu and S. Shankar, "Sensing-Based Opportunistic Channel Access," Mobile Networks and Applications, vol. 11, no. 4, pp. 577-591, August 2006.

[121] M. D. Renzo, L. Imbriglio, F. Graziosi, and F. Santucci, "Distributed Data Fusion over Correlated Log-Normal Sensing and Reporting Channels: Application to Cognitive Radio Networks," IEEE Transactions on Wireless Communications, vol. 8, no. 12, pp. 5813-5821, December 2009.

[122] M. Gudmundson, "A Correlation Model for Shadow Fading in Mobile Radio," Electronic Letters, vol. 27, no. 23, pp. 2145-2146, November 1991.

[123] A. Ghasemi and E. S. Sousa, "Asymptotic Performance of Collaborative Spectrum Sensing under Correlated Log-Normal Shadowing," IEEE Communications Letter, vol. 11, no. 1, pp. 34-36, January 2007.

[124] V. Aalo and R. Viswanathan, "On Distributed Detection with Correlated Sensors: Two Examples," IEEE Transactions on Aerospace and Electronic Systems, vol. 11, no. 4, pp. 414-421, May 1989. 
[125] T. Do and B. L. Mark, "Joint Spatial-Temporal Spectrum Sensing for Cognitive Radio Networks," in Conference on Information Sciences and Systems, Baltimore, MD, March 2009, pp. 124-129.

[126] E. Drakopoulos and C. C. Lee, "Optimum Multisensor Fusion of Correlated Local Decisions," IEEE Transactions on Aerospace and Electronic Systems, vol. 27, no. 4, pp. 593-606, July 1991.

[127] V. Aalo and R. Viswanathan, "Asymptotic Performance of a Distributed Detection System in Correlated Gaussian Noise," IEEE Transactions On Systems, Man, And Cybernetics-Part C: Applications and Reviews, vol. 40, no. 1, pp. 211-213, January 1992.

[128] M. Kam, Q. Zhu, and W. Gray, "Optimal Data Fusion of Correlated Local Decisions in Multiple Sensor Detection Systems," IEEE Transactions on Aerospace and Electronic Systems, vol. 28, no. 3, pp. 916-920, July 1992.

[129] P. Willett, P. F. Swaszek, and R. S. Blum, "The Good, Bad, and Ugly: Distributed Detection of a Known Signal in Dependent Gaussian Noise," IEEE Transactions on Signal Processing, vol. 48, no. 12, pp. 3266-3279, December 2000.

[130] J. G. Chen and N. Ansari, "Adaptive Fusion of Correlated Local Decisions," IEEE Transactions On Systems, Man, And Cybernetics-Part C: Applications and Reviews, vol. 28, no. 2, pp. 276-281, May 1998.

[131] J. Shen, T. Jiang, S. Liu, and Z. Zhang, "Maximum Channel Throughput via Cooperative Spectrum Sensing in Cognitive Radio Networks," IEEE Transactions on Wireless Communications, vol. 8, no. 10, pp. 5166-5175, October 2009.

[132] M. W. Baidas, A. S. Ibrahim, K. G. Seddik, and K. J. R. Liu, "On the Impact of Correlation on Distributed Detection in Wireless Sensor Networks with Relays Deployment," 
in IEEE International Conference on Communications, Dresden, Germany, June 2009, pp. $1-6$.

[133] Y. Liao, "Distributed Decision Fusion in Signal Detection: A Robust Approach," Ph.D. dissertation, Duke University, 2005.

[134] J. H. Holland, Adaptation in Natural and Artificial Systems. The University of Michigan Press, Ann Arbor, 1975.

[135] J. C. Spall, Introduction to Stochastic Search and Optimization. Wiley-Interscience, 2003.

[136] Z. Quan, S. Cui, A. H. Sayed, and H. V. Poor, "Spatial-Spectral Joint Detection for Wideband Spectrum Sensing in Cognitive Radio Networks," in IEEE International Conference on Acoustics, Speech, and Signal Processing, Las Vegas, NV, March 2008, pp. 2793-2796.

[137] L. Khalid, K. Raahemifar, and A. Anpalagan, "Cooperative Spectrum Sensing for Wideband Cognitive OFDM Radio Networks," in IEEE Vehicular Technology Conference, Anchorage, AK, September 2009, pp. 1-5.

[138] A. Sahai and D. Cabric, "A Tutorial on Spectrum Sensing: Fundamental Limits and Practical Challenges," in IEEE International Symposium on New Frontiers in Dynamic Spectrum Access Networks, Baltimore, MD, November 2005.

[139] M. Wylie-Green, "Dynamic Spectrum Sensing by Multiband OFDM Radio for Interference Mitigation," in IEEE International Symposium on New Frontiers in Dynamic Spectrum Access Networks, Baltimore, MD, November 2005, pp. 619-625.

[140] B. Farhang-Boroujeny, "Filter Bank Spectrum Sensing for Cognitive Radios," IEEE Transactions on Signal Processing, vol. 56, no. 5, pp. 1801-1811, May 2008. 
[141] H. Sun, A. Nallanathan, C. X. Wang, and Y. Chen, "Wideband Spectrum Sensing for Cognitive Radio Networks: A Survey," IEEE Wireless Communications, vol. 20, no. 2, pp. 74-80, April 2013.

[142] Y. L. Polo, Y.Wang, and A. Pandharipande, "Compressive Wideband Spectrum Sensing," in IEEE International Conference on Acoustics, Speech, and Signal Processing, Taipei, Taiwan, April 2009, pp. 2337-2340.

[143] F. Zeng, C. Li, and Z. Tian, "Distributed Compressive Spectrum Sensing in Cooperative Multihop Cognitive Networks," IEEE Journal of Selected Topics in Signal Processing, vol. 5, no. 1, pp. 37-48, February 2011.

[144] M. Mishali and Y. C. Eldar, "Blind Multiband Signal Reconstruction: Compressive Sensing for Analog Signals," IEEE Transactions on Signal Processing, vol. 57, no. 3, pp. 993-1009, March 2009.

[145] X. Zhou, J. Ma, G. Y. Li, Y. H. Kwon, and A. Soong, "Probability-Based Combination for Cooperative Spectrum Sensing in Cognitive Radio Networks," in IEEE International Conference on Communications, Dresden, Germany, June 2009, pp. 1-5.

[146] D. B. Cabric, "Cognitive Radios: System Design Perspective," Ph.D. dissertation, University Of California, Berkeley, 2007.

[147] A. P. Engelbrecht, Appendix A: Optimization Theory. John Wiley and Sons, Ltd, 2007.

[148] M. R. Garey and D. S. Johnson, Computers and Intractability: A Guide to the Theory of NP-Completeness. W. H. Freeman, 1979.

[149] C. H. Papadimitriou, Computational Complexity. Addison Wesley, 1994.

[150] C. Y. Huang, C. Y. Lai, and K. T. Cheng, Chapter 4: Fundamentals of Algorithms. Morgan Kaufmann, 2009. 